\title{
Equatorial Coronal Holes and Their Relation to the High-Speed Solar Wind Streams
}

\author{
Dissertation \\ zur Erlangung des Doktorgrades \\ der Mathematisch-Naturwissenschaftlichen Fakultäten \\ der Georg-August-Universität zu Göttingen
}

vorgelegt von
Lidong Xia
aus
Kaihua/China

Göttingen 2003 
D7

Referent: Prof. Dr. Franz Kneer

Korreferent: Prof. Dr. Eckart Marsch

Tag der mündlichen Prüfung: 22. Mai 2003 
Dedicated to Yiling and Minlan 



\begin{abstract}
The heating of the solar corona and the origin and acceleration of the solar wind are among the important unsolved problems of space plasma and solar physics. Coronal holes (CHs) have been known as the source of the fast solar wind. However, the plasma properties at the base of CHs have not yet been fully understood. The purpose of this thesis work is to study equatorial $\mathrm{CHs}$ and their relation to the origin and propagation of the high-speed solar wind streams by combining observations with both space-based (SOHO and WIND) and ground-based (NSO/KP) instruments. With the high spectral and spatial resolution of SUMER on SOHO, the morphology of equatorial CHs was investigated and compared with the quiet-Sun region by deducing 2-D images in ultraviolet emission line parameters (intensity, Doppler shift and width), which provide useful information about the plasma properties in different layers of the solar atmosphere. The relationship between line parameters and the underlying photospheric magnetic field was studied morphologically and statistically. Furthermore, a comparison of coronal and in situ (at $1 \mathrm{AU}$ ) observations was made to study the geometrical expansion factor of the solar wind stream tube. The main findings can be summarized as follows:
\end{abstract}

- The bases of equatorial CHs seen in chromospheric lines generally have similar properties as normal QS regions. An obvious difference has been found in the shape of the H I L $\beta$ line, which has very asymmetric profiles (skewed towards the blue side) in CHs. Loop-like fine structures are the most prominent features in the transition region.

- Apparent blue shifts are found in Dopplergrams deduced from transition region lines formed at a temperature below $510^{5} \mathrm{~K}$ (although on average they are red shifted). Structures with bluer shifts usually have also broader line widths. They seem to represent plasma above large concentrations of unipolar magnetic field, without obvious bipolar photospheric magnetic features nearby.

- Blue shifts deduced from the Ne VIII $\left(T_{e} \approx 6.310^{5} \mathrm{~K}\right)$ and $\operatorname{Mg} \mathrm{X}\left(T_{e} \approx 1.110^{6} \mathrm{~K}\right)$ lines predominate in the $\mathrm{CH}$ region. Larger-scale outflow are mainly associated with the network where unipolar magnetic field dominates (open magnetic funnels). Red or less blue shifts in EUV bright points indicate that they are unlikely the main source of the fast solar wind.

- The Mg X line broadening shows a clear trend to increase with the increasing magnetic field strength. The spectroscopically obtainable quantity of $v \sqrt{I}$ (with $I \sim n_{e}^{2}$ ), which is used as a proxy for the coronal mass flux of the nascent fast solar wind, also reveals a clear positive correlation to the magnetic field strength.

- Expansion factors of the solar wind stream tube are determined consistently from two independent groups of parameters, relating to the conservation of the mass flux and magnetic flux, respectively.

The observational results concerning the source of the fast solar wind in CHs obtained in this work are expected to provide a clearer physical picture of the plasma conditions prevailing at the coronal hole base, and thus to be important constraints on theory. 



\section{Publications and contributions}

Parts of the results of this thesis were taken from the following publications and conference contributions.

\section{Publications}

L. D. Xia and E. Marsch, Equatorial Coronal holes and Their Relation to the High-Speed Solar Wind Streams, in Conference Proceeding: "Solar Wind Ten", American Institute of Physics (AIP), p.319-322, 2003.

L. D. Xia, E. Marsch and W. Curdt, On the Outflow in an Equatorial Coronal Hole, A\&A, 399, L5-L9, 2003.

\section{Conference contributions}

L. D. Xia, E. Marsch, I. E. Dammasch and K. Wilhelm, SUMER Observations of Coronal Holes on the Disk, XXVI General Assembly of the European Geophysical Society, Nice, France, 25 - 30 March, 2001 (oral report).

L. D. Xia and E. Marsch, Equatorial Coronal Holes and Their Relation to Solar Wind High-Speed Streams, Solar Wind 10, Pisa, Italy, 17-21 June, 2002 (poster). 



\section{Contents}

Contents i

List of Figures iv

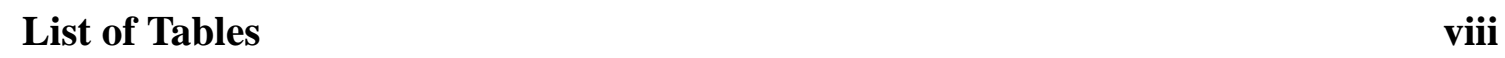

\begin{tabular}{ll}
\hline List of Abbreviations & xi
\end{tabular}

1 Introduction 1

1.1 The Sun and solar wind . . . . . . . . . . . . . . . . . . . . 1

1.2 Great puzzle: coronal heating and solar wind acceleration . . . . . . . . . 4

1.3 Outline of the thesis . . . . . . . . . . . . . . . . . . 4

2 Review: Coronal Holes and Origin of the Fast Solar Wind 5

$2.1 \quad$ Overall description of coronal holes . . . . . . . . . . 5

$2.1 .1 \quad$ Introduction . . . . . . . . . . . . . . . . . 5

2.1 .2 Coronal holes seen in various wavelengths . . . . . . . . 6

2.1 .3 The morphology of coronal holes . . . . . . . . . . . . 7

2.1 .4 Underlying photospheric magnetic fields . . . . . . . . . . 9

2.1 .5 Fine structures in coronal holes . . . . . . . . . . . . . . 10

2.2 FUV/EUV observations of coronal holes . . . . . . . . . . . . . . . 15

2.2 .1 Observations of FUV/EUV radiance . . . . . . . . . . . . . . 15

2.2 .2 Underlying chromosphere and transition region . . . . . . . . . 16

2.3 Plasma parameters deduced from observations . . . . . . . . . . . . . 17

2.3 .1 Plasma velocity inferred by Doppler shifts . . . . . . . . . . . . 17

2.3 .2 Non-thermal velocity . . . . . . . . . . . . . . . . . . . . . . . . . . . 20

2.3 .3 The density and temperature . . . . . . . . . . . . 21

2.4 Coronal holes and the fast solar wind . . . . . . . . . . . . . . . . . . 22

2.4 .1 The fast solar wind . . . . . . . . . . . . . . . . . 22

2.4 .2 Coronal holes: sources of the fast solar wind . . . . . . . . 26

2.5 Heating and acceleration mechanisms in coronal holes . . . . . . . . . . 27

2.5 .1 The mass and energy balance . . . . . . . . . . . . 27

2.5 .2 Heating and acceleration mechanisms . . . . . . . . . . . . . . . . . . . . . . . 39

2.5 .3 Recent modelling studies of the coronal funnels . . . . . . . . . 31 
3 Instrumentation and Diagnostic Principles 3

3.1 Introduction . . . . . . . . . . . . . . . . . 33

3.1 .1 Overview of observations at FUV/EUV wavelengths . . . . . . . 33

3.1 .2 SOHO mission . . . . . . . . . . . . . . . . 34

3.2 The SUMER instrument . . . . . . . . . . . . . . . 36

3.2 .1 Scientific goals of SUMER . . . . . . . . . . . . . 36

3.2 .2 The SUMER spectrometer . . . . . . . . . . . . . . 37

3.2 .3 Calibrations and corrections . . . . . . . . . . . . 42

3.3 Additional instruments . . . . . . . . . . . . . . . . . 45

3.3 .1 EIT instrument . . . . . . . . . . . . . . . . 45

3.3 .2 MDI instrument . . . . . . . . . . . . . . . . . 45

3.3 .3 NASA/NSO Spectromagnetograph. . . . . . . . . . . . . 45

3.3 .4 SWE instrument . . . . . . . . . . . . . . . . . . 46

3.3 .5 MFI instrument . . . . . . . . . . . . . . . . 46

3.4 Diagnostic methods with FUV/EUV lines . . . . . . . . . . . . . 46

3.4.1 Atomic processes in the upper solar atmosphere . . . . . . . . 46

3.4 .2 Formation of line and continuum emission . . . . . . . . . . 48

3.4 .3 Diagnostics with FUV/EUV lines . . . . . . . . . . . . . . . . 49

4 Observations and Methods of Data Analysis 55

4.1 Description of observations . . . . . . . . . . . . . . . . 55

4.2 Identification of coronal holes . . . . . . . . . . . . . . . 58

4.3 Identification of lines $\ldots \ldots \ldots \ldots$. . . . . . . . . . . . 61

4.4 Determination of line parameters: intensity, position and width . . . . . . 62

4.4 .1 Discussion of estimating errors . . . . . . . . . . . . . . . . 64

4.5 Additional geometrical correction . . . . . . . . . . . . . . . 65

4.6 Wavelength calibration $\ldots \ldots \ldots \ldots 6$

5 Morphology of the Equatorial Coronal Holes 69

5.1 Introduction . . . . . . . . . . . . . . . . . . . 69

5.2 Data selection . . . . . . . . . . . . . . . . . . . . . 69

5.3 Spectroheliograms . . . . . . . . . . . . . 71

5.3 .1 Chromospheric lines and continua . . . . . . . . . 71 
5.3 .2 Transition region lines $\left(T_{e}<0.5 \mathrm{MK}\right) \ldots \ldots . \ldots 72$

5.3 .3 Upper transition region and coronal lines $\left(T_{e}>0.5 \mathrm{MK}\right)$. . . . 73

5.4 Doppler shifts . . . . . . . . . . . . . . . . . . . 79

$5.4 .1 \quad$ Chromospheric and transition region lines $\left(T_{e}<0.5 \mathrm{MK}\right)$. . . 79

5.4 .2 Upper transition region and coronal lines $\left(T_{e}>0.5 \mathrm{MK}\right)$. . . . 81

5.5 Network, bright structures and underlying magnetic fields . . . . . . . . . 81

5.6 Outflow at the coronal base . . . . . . . . . . . . . . . . . 87

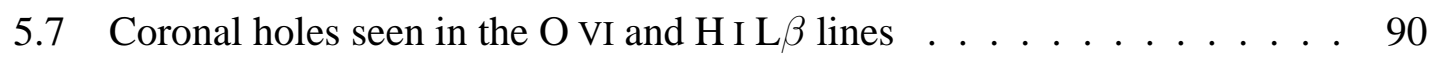

$5.7 .1 \quad$ Analysis of the $\mathrm{H} \mathrm{I} \mathrm{L} \beta$ line . . . . . . . . . . . . . . 990

5.7 .2 Loop-like structures seen in the O VI line . . . . . . . . . . . 95

5.8 Rapid time variations $\ldots \ldots \ldots$. . . . . . . . . . . . . 101

5.9 Case study of a transient event . . . . . . . . . . . . . . . 103

5.10 Summary and discussion . . . . . . . . . . . . . . . 105

$\begin{array}{lll}6 & \text { Statistical and Quantitative Analysis } & 109\end{array}$

6.1 Introduction . . . . . . . . . . . . . . . . . . . . . . . . . . 109

6.2 Data selection and analysis $\ldots \ldots \ldots$. . . . . . . . . . . 110

6.3 Statistical studies of line parameters . . . . . . . . . . . . . . 111

6.3.1 Distribution of line intensity, Doppler shift and width . . . . . . . 111

6.3.2 Relationship between line parameters and the chromospheric net-

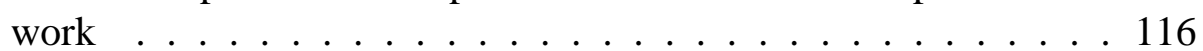

6.3 .3 Relationship between Doppler shifts of different lines. . . . . . . 121

6.4 Average intensity, Doppler shift and line width . . . . . . . . . . . . 122

$6.4 .1 \quad$ Lines with formation temperatures $T_{e}>0.5 \mathrm{MK} \ldots 123$

6.4 .2 Transition region lines . . . . . . . . . . . . . . 127

6.4 .3 Chromospheric lines . . . . . . . . . . . . . 135

6.5 Temperature dependence of intensity, Doppler shift and line width . . . . 138

$6.5 .1 \quad$ Intensity ratio between the $\mathrm{CH}$ and $\mathrm{QS}$ regions . . . . . . . 138

6.5 .2 Average Doppler shift . . . . . . . . . . . . . . . 139

6.5 .3 Average line width . . . . . . . . . . . . . . . 141

6.6 Plasma parameters deduced from coronal lines and relation to the underlying magnetic field . . . . . . . . . . . . . . . . 143

$6.7 \quad$ Summary and discussion $\ldots \ldots \ldots$. . . . . . . . . . . . . 145 
\begin{tabular}{|ll|}
\hline 7 & Comparison of Coronal and in situ Observations \\
\hline
\end{tabular}

7.1 Introduction . . . . . . . . . . . . . . . . . . . . . 147

7.2 Data selection . . . . . . . . . . . . . . . . . . . . . . . . 148

7.3 Equatorial coronal holes and high-speed solar wind streams . . . . . . . . 148

7.4 Diagnostic of electron density in coronal holes . . . . . . . . . . . . . 152

7.4 .1 Line intensity ratios . . . . . . . . . . . . . 152

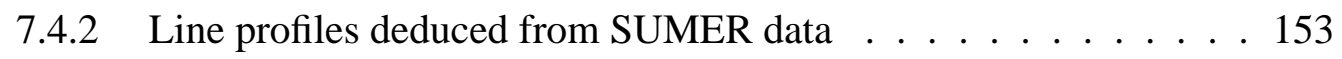

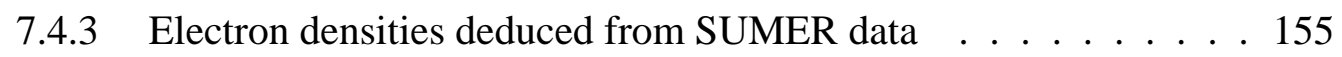

7.5 Comparison of coronal and in situ observations . . . . . . . . . . . 158

7.6 Summary and discussion . . . . . . . . . . . . . . . . . 159

8 Discussion: Implication to the Origin of the Fast Solar Wind 161

8.1 Fine structures and the network in coronal holes . . . . . . . . . . . . . . 161

$8.2 \quad$ Fine structures, spicules and plumes . . . . . . . . . . . . . . 162

8.3 Doppler shifts and the nascent fast solar wind . . . . . . . . . . . . . 163

8.4 Line width and heating mechanism . . . . . . . . . . . . . . . 165

8.5 Magnetic field and the global fast solar wind . . . . . . . . . . . . . . 166

\begin{tabular}{lll}
\hline 9 & Summary & 167
\end{tabular}

\begin{tabular}{ll}
\hline Bibliography & 171
\end{tabular}

\begin{tabular}{lr}
\hline Danksagung & 189
\end{tabular}

\begin{tabular}{ll}
\hline Lebenslauf & 191
\end{tabular} 


\section{List of Figures}

1.1 The solar atmosphere: temperature and density profiles (source: Mariska,

1.2 The solar corona and solar wind (source: McComas et al., 2000) . . . . . 3

$2.1 \quad$ SUMER observation: different types of the coronal hole. . . . . . . . . . 5

2.2 The formation of the coronal hole (source: Bohlin and Sheeley, 1978) . . 8

2.3 Gabriel's network model (source: Gabriel, 1976) . . . . . . . . . . . . . 11

2.4 Dowdy's network model (source: Mariska, 1992) . . . . . . . . . . . 11

2.5 Observations of Doppler shifts in the QS region (source: Brekke, 1999) $\quad 19$

2.6 Observations of non-thermal velocities in the QS region (source: Chae,

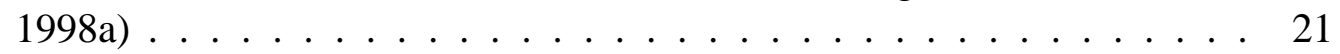

2.7 Density profile vs. heliocentric distance (source: Sittler and Guhathakurta, 1999; Doyle et al., 1999) . . . . . . . . . . . . . . . 24

2.8 Properties of the fast solar wind near the Sun (source: Cranmer, 2002) . . 25

2.9 SWICS/Ulysses observations: solar wind speed vs. heliographic latitude (source: Woch et al., 1997) . . . . . . . . . . . . . . . 27

2.10 SUMER observation: the origin of the fast solar wind (source: SUMER

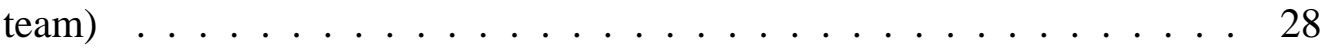

2.11 Magnetic furnace (source: Axford et al., 1999) . . . . . . . . . . . . . . 30

$3.1 \quad$ Selected emission lines in wavelength range covered by SUMER and other instruments (source: Wilhelm et al., 1995) . . . . . . . . . . . . . 37

3.2 The optical layout of the SUMER instrument (source: SUMER team) . . 38

3.3 Detector arrays in the focal plane of the grating (source: Wilhelm et al.,

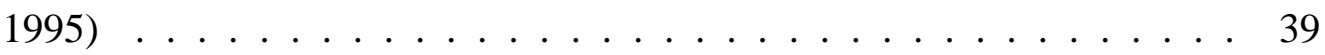

3.4 The sensitivities of SUMER as obtained during the radiometric calibration (source: Wilhelm 2003) _ . . . . . . . . . . . . . . . . 44

4.1 Context of the SUMER observation of a CH on 11 March, 1999 . . . . . 59

4.2 Context of the SUMER observation of a CH on 19 October, 1999. . . . . 60

4.3 Context of the SUMER observation of a QS region on 8 March,1999 . . . 61

4.4 Comparison of two methods for deducing the line parameters . . . . . . . 64 
4.5 Residual errors of the line position after the geometrical correction using the standard software . . . . . . . . . . . . . . 65

$5.1 \mathrm{CH} / \mid$ as seen in the EIT $304 \AA, 171 \AA, 195 \AA$ and $284 \AA$ channels $\mid . . . . \quad 70$

5.2 Intensity maps obtained in the spectral window $1532 \AA$ to $1552 \AA$. . . . 74

5.3 Intensity maps obtained in the spectral window $1233 \AA$ to $1253 \AA$. . . . 75

5.4 Intensity maps obtained in the spectral window $1160 \AA$ to $1180 \AA$. . . . 76

5.5 Intensity maps obtained in the spectral window $915 \AA$ to $935 \AA$. . . . . . . 77

$5.6 \quad$ Intensity maps obtained in the spectral window $1525 \AA$ to $1565 \AA$. . . . 78

5.7 Intensity maps obtained in the spectral window $1525 \AA$ to $1545 \AA$. . . . 78

5.8 Dopplergrams of chromospheric lines for $\mathrm{CH} 6$ | observed on 11, 12 and 13 March, 1999 . . . . . . . . . . . . . . . . 80

5.9 Dopplergrams of transition region and coronal lines for $\mathrm{CH}$ (6) observed on 11 and 12 March, 1999 . . . . . . . . . . . . . . 80

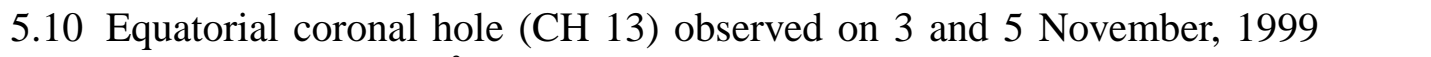

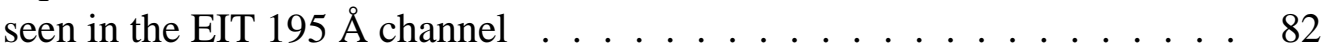

5.11 Network and bright points seen in different lines observed by SUMER, EIT and NSO/KP on 5 November, 1999 . . . . . . . . . . . . . 83

5.12 The evolution of the bright points seen in magnetograms observed by MDI 84

\begin{tabular}{|ll||l|l|l|}
$5.13 \mathrm{CH}$ & 13 & seen in the 4 channels observed by EIT on 3 November, 1999 & . . . 85 \\
\hline
\end{tabular}

$5.14 \mathrm{CH} 13$ seen in the continuum, Si II, C IV, and Ne VIII lines observed by SUMER on 3 November, 1999 . . . . . . . . . . . . . . . . . 86

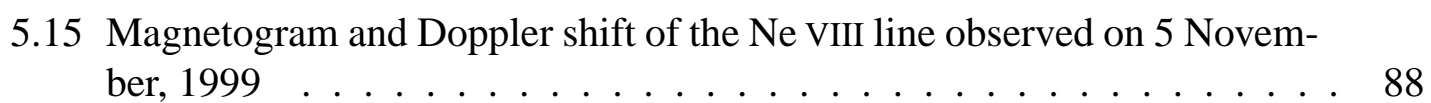

5.16 Doppler shifts of the Ne VIII line observed on 3 November, 1999 . . . . . 88

5.17 Line profiles of the H I L-11 and $\mathrm{H} \mathrm{I} \mathrm{L} \beta$ lines in $\mathrm{CH}$ and QS regions ... 91

5.18 Images in intensity and Doppler shift of the $\mathrm{H} \mathrm{I} \mathrm{L} \beta$ line in $\mathrm{CH}$ and QS

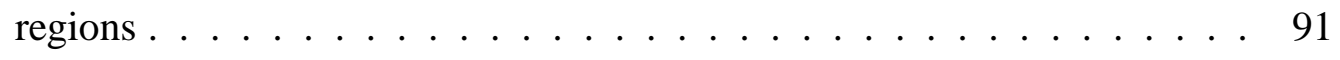

5.19 Analysis of hydrogen $\mathrm{L} \beta$ line profiles in $\mathrm{CH}$ and $\mathrm{QS}$ regions . . . . . . 92

$5.20 \mathrm{CH}$ images in intensity and Doppler shift showing fine structures, observed on 8 November, $1999 \ldots \ldots . \ldots . \ldots 96$

$5.21 \mathrm{CH}$ images in intensity and Doppler shift showing fine structures, observed on 11 March, $1999 \ldots \ldots \ldots$. . . . . . . . . . 97

5.22 Fine structures in $\mathrm{CH}$ and $\mathrm{QS}$ regions seen in the $\mathrm{O} \mathrm{VI}$ line . . . . . . . . 98

5.23 An ECH observed on 19 October, 1996 seen in the EIT $195 \AA$ channel . . 101 
5.24 Images in intensity and line shift in the spectral window $760 \AA$ to $795 \AA$ with an exposure time of $60 \mathrm{~s} \ldots \ldots \ldots 103$

5.25 Images in intensity and line shift in the spectral window $760 \AA$ to $795 \AA$ with an exposure time of $20 \mathrm{~s} \ldots \ldots \ldots$. . . . . . . . . . . . . . . .

5.26 Spectral window from $1234 \AA$ to $1254 \AA$ showing a transient event . . . . 105

5.27 Line profile in the spectral window from $1234 \AA$ to $1254 \AA$ showing a transient event . . . . . . . . . . . . . . 106

6.1 Histograms of chromospheric continuum at $1539 \AA, 1235 \AA$ and $912 \AA$. 112

6.2 Histograms of integrated line intensity, Doppler shift and line width (Si II and $\left.\mathrm{He}_{\mathrm{I}}\right] \ldots \ldots \ldots \ldots . \ldots \ldots \ldots$

6.3 Histograms of integrated line intensity, Doppler shift and line width (C $\mathrm{C}_{\text {II }}$ and $\left.\mathrm{C}_{\text {IV }}\right) \ldots \ldots \ldots \ldots \ldots \ldots \ldots \ldots$

6.4 Histograms of integrated line intensity, Doppler shift and line width (S VI

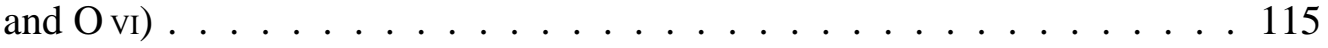

6.5 Histograms of integrated line intensity, Doppler shift and line width (Ne VIII and $\mathrm{Mg}$ x) . . . . . . . . . . . . . . . . 116

$6.6 \quad$ Line parameters and their relation to the chromospheric network (Si II and

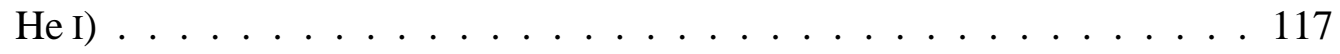

$6.7 \quad$ Line parameters and their relation to the chromospheric network (C II and

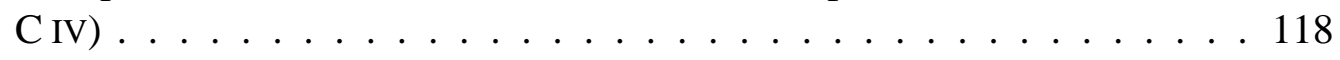

6.8 Line parameters and their relation to the chromospheric network (C II and C IV) . . . . . . . . . . . . . . . . . . . . . . 119

6.9 Line parameters and their relation to the chromospheric network (Ne VIII

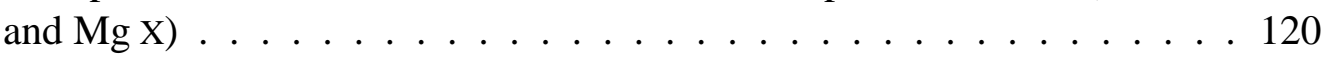

6.10 Relationship between Doppler shifts of different lines ( $\mathrm{H}$ I L $\beta, \mathrm{C}$ II and O

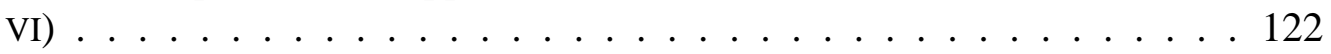

6.11 Line profiles of the $\mathrm{Mg} X$ line with its reference lines of C I nearby . . . . 123

6.12 Averaged line profile of the Ne VIII $(770 \AA)$ in $\mathrm{CHs}$. . . . . . . . . . . 125

6.13 Line profiles of the N V, S VI and O V lines at $1239 \AA, 933 \AA$ and $1032 \AA 128$

6.14 Line intensity ratio between $\mathrm{CH}$ and QS regions in various lines vs. line formation temperature . . . . . . . . . . . 138

6.15 Average Doppler shift in various lines vs. line formation temperature in CHs and QS . . . . . . . . . . . . . . . . 140

6.16 Average line width in various lines vs. line formation temperature in $\mathrm{CHs}$

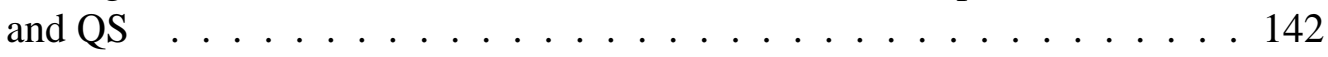


6.17 Line parameters of the $\mathrm{Mg} \mathrm{X}$ line vs. magnetic field strength observed in

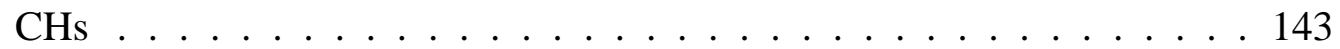

$7.1 \quad$ EIT meridian map during the period from 22 August to 3 September, 1996149

7.2 EIT meridian map during the period from 8 October to 1 November, 1996149

7.3 Solar wind parameters at 1 AU during 22 August and 3 September, 1996. 150

7.4 Solar wind parameters at 1 AU during 8 October and 1 November, 1996 . 151

7.5 Theoretical line intensity ratios of line pairs Si III, O V and Mg IX . . . . . 154

7.6 EIT map on 27 August, 1996, showing part of the coronal hole "Elephant's Trunk" . . . . . . . . . . . . . . . 155

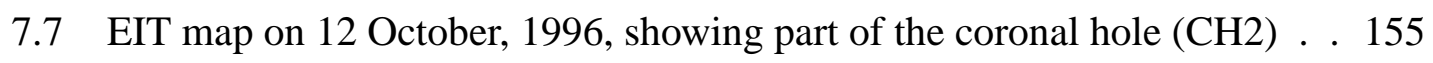

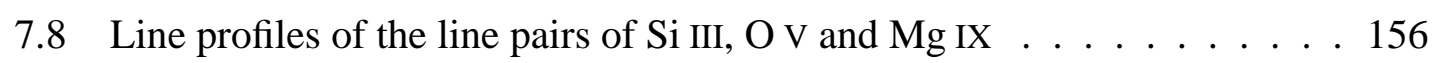




\section{List of Tables}

$2.1 \quad$ Two modes of solar wind flow (source: Axford and McKenzie, 1997) . . . 23

2.2 Chromospheric and coronal energy losses (source: Withbroe, 1977) . . . 29

$3.1 \quad$ The SOHO scientific instruments (source: Domingo et al., 1995) . . . . . 35

3.2 The performance of SUMER instrument (source: SUMER team) . . . . . 41

4.1 SUMER observations of coronal holes on the disk . . . . . . . . . . 56

4.2 SUMER observations of QS regions near the disk center . . . . . . . . 57

4.3 Emission lines selected for this study . . . . . . . . . . . . . . . . 62

4.4 Comparison of two methods for deducing the line parameters . . . . . . . 64

$5.1 \quad$ Derived line parameters for the $\mathrm{H} \mathrm{I} \mathrm{L} \beta$ line $\ldots \ldots \ldots$. . . . . . . . . . . . . 94

6.1 Derived line parameters for the Mg X line . . . . . . . . . . . . . . . . . . . . . . 124

6.2 Derived line parameters for the Ne VIII line . . . . . . . . . . . . 127

6.3 Derived line parameters for the O VI line . . . . . . . . . . . . . . . 129

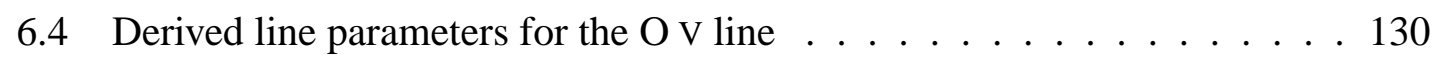

6.5 Derived line parameters for the $\mathrm{S}$ VI line . . . . . . . . . . . . . 131

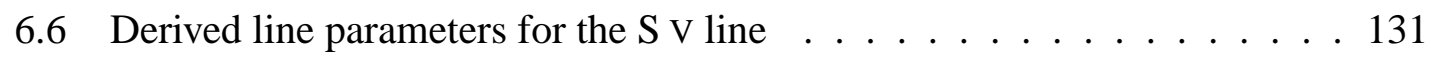

6.7 Derived line parameters for the $\mathrm{N} V$ line . . . . . . . . . . . . . . . 132

6.8 Derived line parameters for the C IV line . . . . . . . . . . . . . . 133

6.9 Derived line parameters for the N IV line . . . . . . . . . . . . . . . . . . . . . . . . . . . . . .

6.10 Derived line parameters for the O IV line . . . . . . . . . . . . . . . 134

6.11 Derived line parameters for the Si IV line . . . . . . . . . . . . . . . . 134

6.12 Derived line parameters for the $\mathrm{C}$ II line . . . . . . . . . . . . . 135

6.13 Derived line parameters for the Si II line . . . . . . . . . . . . . 136

6.14 Derived line parameters for the O I line . . . . . . . . . . . . . . 137

6.15 Derived intensity of the continuum . . . . . . . . . . . . . 138

7.1 Instruments and their measurement items: comparison of coronal and in situ observations . . . . . . . . . . . . . . . . . . . . 148

7.2 Electron densities deduced from SUMER data . . . . . . . . . . . . . . . 157

7.3 Coronal parameters deduced from SUMER, MDI and NSO/Kitt Peak . . 158

7.4 In-situ parameters deduced from SWE and MFI . . . . . . . . . . . . 159 


\section{List of Abbreviations}

\section{Spacecraft and instrument abbreviations}

$\begin{array}{ll}\text { ACE } & \text { Advanced Composition Explorer } \\ \text { ATM } & \text { Apollo Telescope Mount } \\ \text { CDS } & \text { Coronal Diagnostic Spectrometer } \\ \text { CELIAS } & \text { Charge, ELement and Isotope Analysis System } \\ \text { EIT } & \text { Extreme-ultraviolet Imaging Telescope } \\ \text { ERNE } & \text { Energetic and Relativistic Nuclei and Electron experiment } \\ \text { GOLF } & \text { Global Oscillations at Low Frequencies } \\ \text { HCO } & \text { Harvard College Observatory } \\ \text { HRTS } & \text { High Resolution Telescope and Spectrograph } \\ \text { IMP } & \text { Interplanetary Monitoring Platform } \\ \text { LASCO } & \text { Large Angle and Spectrometric COronagraph } \\ \text { LASP } & \text { Laboratory for Atmospheric and Space Physics } \\ \text { MCS } & \text { Multichannel Spectrometer } \\ \text { MDI/SOI } & \text { Solar Oscillations Investigation/Michelson Doppler Imager } \\ \text { MFI } & \text { Magnetic Field Investigation } \\ \text { NSO/KP } & \text { National Solar Observatory at Kitt Peak } \\ \text { OSO } & \text { Obiting Solar Observatory } \\ \text { SMM } & \text { Solar Maximum Mission } \\ \text { SOHO } & \text { Solar and Heliospheric Observatory } \\ \text { SUMER } & \text { Solar Untraviolet Measurements of Emitted Radiation } \\ \text { SWAN } & \text { Solar Wind ANisotropies } \\ \text { SWE } & \text { Solar Wind Experiment } \\ \text { SWICS } & \text { Solar Wind Ion Composition Spectrometer } \\ \text { SXT } & \text { Soft X-ray Telescope } \\ \text { TRACE } & \text { Transition Region And Coronal Explorer } \\ \text { UVCS } & \text { UltraViolet Coronagraph Spectrometer } \\ \text { UVS } & \text { Ultraviolet Spectrometer } \\ \text { UVSP } & \text { Ultraviolet Spectrometer and Polarimeter } \\ \text { VIRGO } & \text { Variability of solar IRradiance and Gravity Oscillations } \\ & \\ \text { SW } & \end{array}$

\section{Other abbreviations}

$\begin{array}{ll}\text { AR } & \text { Active region } \\ \text { AU } & \text { Astronomical unit } \\ \text { BP } & \text { Bright point } \\ \text { CH } & \text { Coronal hole } \\ \text { DEM } & \text { Differential emission measure }\end{array}$




$\begin{array}{ll}\text { DQE } & \text { Detection quantum efficiency } \\ \text { ECH } & \text { Equatorial coronal hole } \\ \text { EE } & \text { Explosive event } \\ \text { EUV } & \text { Extreme-ultraviolet } \\ \text { FOV } & \text { Field of view } \\ \text { FUV } & \text { Far-ultraviolet } \\ \text { GSE } & \text { Geocentric Solar Ecliptic } \\ \text { IMF } & \text { Interplanetary magnetic field } \\ \text { IPS } & \text { Interplanetary scintillation } \\ \text { KBr } & \text { Potassium Bromide } \\ \text { LOS } & \text { Line-of-sight } \\ \text { MCP } & \text { Microchannel Plates } \\ \text { MHD } & \text { Magnetohydrodynamics } \\ \text { PCH } & \text { Polar coronal hole } \\ \text { QS } & \text { Quiet Sun } \\ \text { RSC } & \text { Rear slit camera } \\ \text { UMR } & \text { Unipolar magnetic region } \\ \text { UTC } & \text { Coordinated universal time } \\ \text { UV } & \text { Ultraviolet } \\ \text { VUV } & \text { Vacuum-ultraviolet } \\ \text { XDL } & \text { Cross-delay-line }\end{array}$




\section{Chapter 1}

\section{Introduction}

\subsection{The Sun and solar wind}

Although the Sun, our star, is an ordinary G2 main sequence star, it is the only star in the universe close enough to us to be studied in detail. The Sun is a huge gas ball with a radius of about $6.96 \times 10^{5} \mathrm{~km}$, a mass of about $1.99 \times 10^{33} \mathrm{~kg}$, a surface temperature of about $5770 \mathrm{~K}$ and a luminosity of about $3.85 \times 10^{26} \mathrm{~W}$. It consists mainly of hydrogen (about $90 \%$, by number) and helium (about $10 \%$ ) and other minor components (about $0.1 \%$ ). The huge radiation energy of the Sun originally comes from its center part $\left(0 \sim 0.25 \mathbf{R}_{\odot}\right)$, the Sun's core, where nuclear reactions consume hydrogen to form helium. This energy is transported outward through the radiative zone $\left(0.25 \sim 0.75 \mathrm{R}_{\odot}\right.$ ) by radiation (mostly gamma-rays and $\mathrm{X}$-rays), and then through the outermost convection zone $\left(0.75 \sim 1 \mathrm{R}_{\odot}\right)$ by convective motions of fluid flows.

The Sun has no solid surface, however, the outer layer above the convection zone is defined as the solar atmosphere, which can be observed directly by optical means. According to its physical properties, we divide further the solar atmosphere into four layers, namely, the photosphere, the chromosphere, the transition region and the corona. The temperature and density profiles, which vary with height above the photosphere are shown in Figure 1.1 .

The photosphere is a thin layer above the convection zone with a thickness of about 400 $\mathrm{km}$. It is the transition layer between the interior and the outer atmosphere of the Sun, and the only layer of the Sun which can be seen by human eyes. The most solar radiation emitted into interplanetary space comes directly from this layer. Because of this, the gas becomes cooler and its temperature reaches a minimum of about $4500 \mathrm{~K}$ at the top of the photosphere. The main features that can be readily observed in this layer are the dark sunspots, bright faculae, and small granules. Additional patterns, which include supergranules, large-scale flows and oscillations, can be found by studying Doppler shifts of the photospheric material.

The chromosphere is the region above the photosphere where the temperature rises from 4500 to about $20,000 \mathrm{~K}$. The chromosphere gets its name from its apparent reddish colour $\left(\mathrm{H}_{\alpha}\right.$ emission) during total solar eclipses. A variety of features can be observed in chromosphere. Among them, the chromospheric network associated with the boundaries of supergranular cells and jet-like spicules above the limb are the basic structures in the quiet Sun. Other features include bright plages around sunspots, dark filaments across the disk, and prominences above the limb.

The transition region is traditionally defined as the interface between the hot corona and much cooler chromosphere, where the temperature changes rapidly from about 20,000 K 


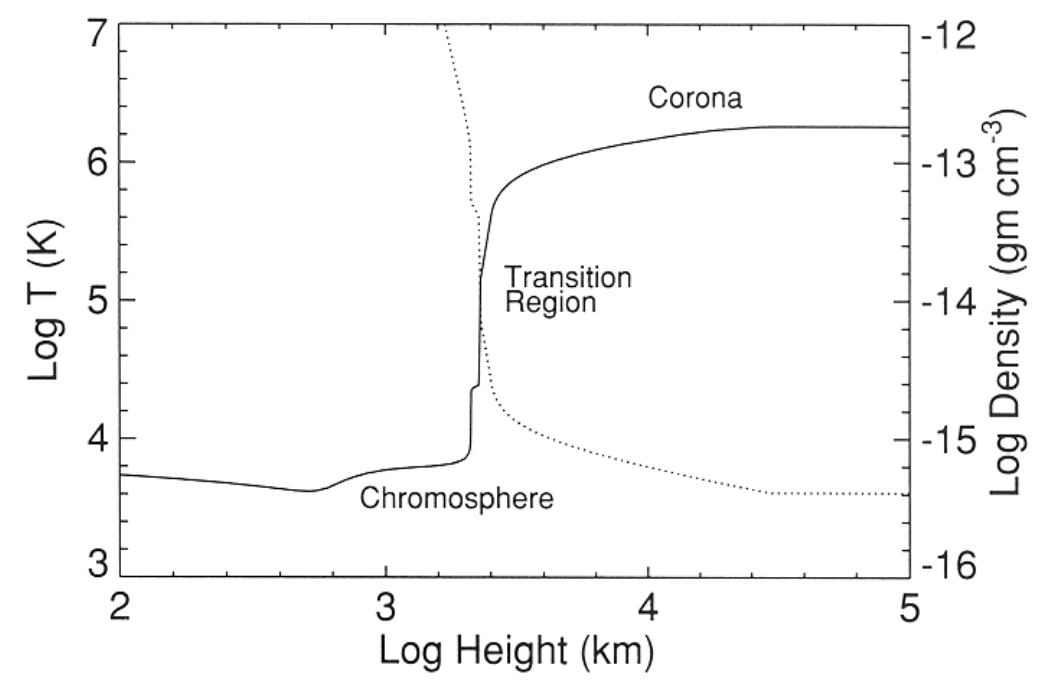

Figure 1.1: The average temperature and density profiles varying with height above the photosphere show that temperature and density change dramatically from the chromosphere to the coronal base. After Mariska (1992)

up to $1 \mathrm{MK}$ (million Kelvin). A large number of UV/EUV lines emitted by the transition region can be observed. Detailed physical properties of this layer still remain unclear. Recent studies show that the transition region is dominated by fine loop-like structures.

The corona is the outer atmosphere of the Sun. It is visible only during total eclipses by human eyes. This coronal light contains different spectral contributions: K-corona contributed by photospheric light scattered off coronal electrons; F-corona resulting from the Fraunhofer lines diffracted by the dust in interplanetary space; E-corona originating by the atomic emission from the hot plasma. The corona is structured in various features including coronal holes, streamers, plumes, and loops. In the corona, the plasma has a high temperature in excess of $1 \mathrm{MK}$, and is highly diluted. The magnetic field extending from the chromospheric network becomes more homogeneous in this layer. The field lines channel the mechanical energy and the plasma flow. Consequently, the overall shape of the corona changes with the sunspot cycle, which is directly related to the change of the magnetic topology of the Sun (see upper panel in Figure 1.2).

The hot corona extends radially outward into interplanetary space. The flow quickly becomes supersonic within several solar radii to form the solar wind, which permeates then into the whole interplanetary space. At the same time, the solar magnetic field lines are dragged by the flow due to the high electrical conduction of the wind plasma, and the solar rotation is, at a sidereal period of about 25 days, leading to a spiral configuration of the magnetic field. The main component of the solar wind are protons and electrons with an admixture of a few percent of alpha particles and heavy, much less abundant ions in different ionization stages. The solar wind was theoretically predicted by Parker (1958) and confirmed by direct measurements in the early 1960's (Gringauz et al., 1960; Snyder and Neugebauer, 1963; Neugebauer and Snyder, 1966). In Figure 1.2 (bottom panel), 


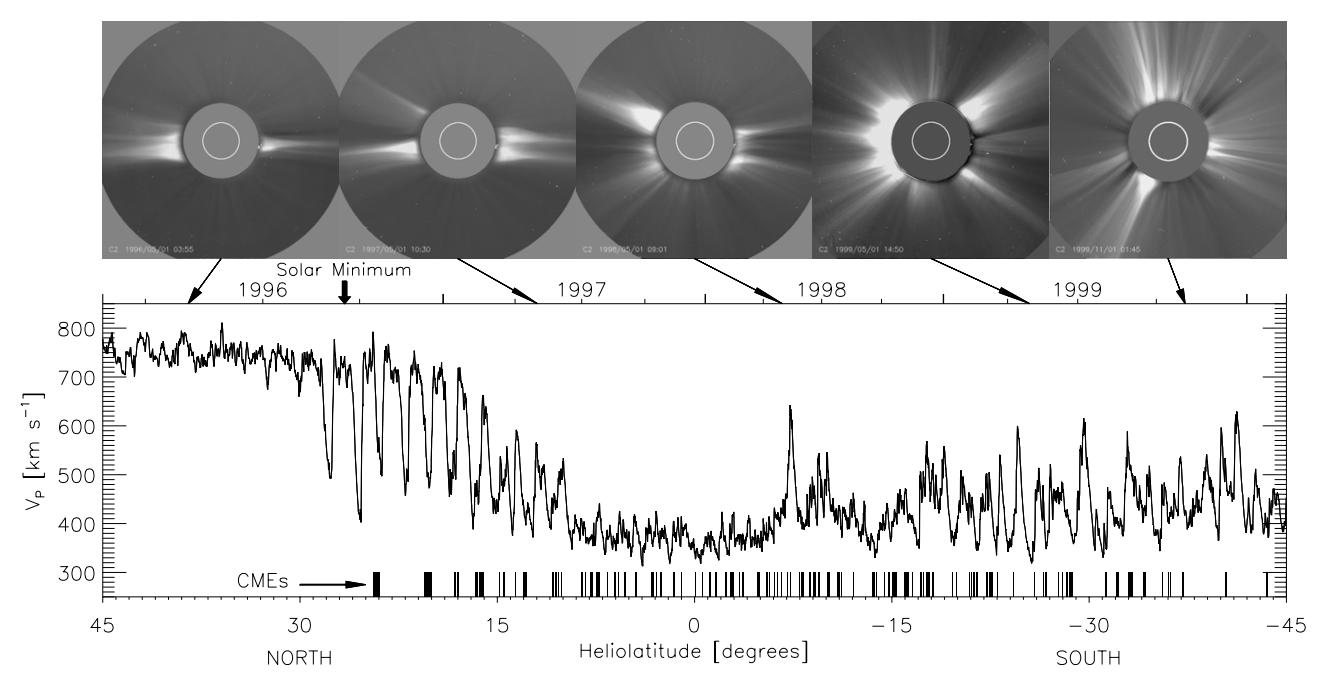

Figure 1.2: The shape of the solar corona changing in time as seen by the LASCO coronagraph on $\mathrm{SOHO}$ and the associated solar wind flow velocity versus heliographic latitude as measured in situ on Ulysses. Note that the Sun reached its past activity minimum in 1996 and maximum in 2000. After McComas et al. (2000)

the solar wind flow velocity is plotted versus heliographic latitude as measured in situ on Ulysses. Characterized by their different flow velocities, two basic modes of the solar wind, namely, the slow and fast wind, can be seen in this plot. The fast wind has average velocity of about $750 \mathrm{~km} \mathrm{~s}^{-1}$, while the slow wind has a typical velocity less than $400 \mathrm{~km}$ $\mathrm{s}^{-1}$.

The earliest observations of the solar atmosphere could be retrospected to about 2000 years ago, when the ancient Chinese began to record their observations of sunspots and eclipses. However, modern optical observations of the Sun started at the beginning of 17th century, when Galileo used the telescope to observe sunspots and Newton found the prismatic spectrum of the visible sunlight. Since the beginning of 20th century, the spectral analysis has become a very important method to study the solar atmosphere. Moreover, earlier studies of the corona were usually made with the help of eclipses. Today, we can produce artificial eclipses in coronagraphs that cover the disk of the Sun in order to obtain the emission coming only from coronal ions. These coronagraphs produce images of the E-corona. Radiation in FUV/EUV and X-ray wavelengths originate in the solar atmosphere mainly above the photosphere. This allows us to view the upper solar atmosphere against the disk of the Sun. However, radiation in these wavelengths can only be observed above the absorbing Earth's atmosphere. A large number of instruments onboard spacecrafts and rockets have been developed, such as instruments on spacecrafts OSO, Skylab, SMM, Spacelab and recent Yohkoh, SOHO and TRACE. For the solar wind, various in situ observations have been carried out on spacecrafts such as Helios, IMP, WIND, Ulysses, ACE and SOHO. 


\subsection{Great puzzle: coronal heating and solar wind accel- eration}

One of the most surprising discoveries in solar physics was the high temperature of the corona. Early observations of the visible spectrum of the corona revealed bright emission lines at wavelengths that did not correspond to any known materials. This led astronomers to propose the existence of "coronium" as the principal gas in the corona. In 1939, the mystery was solved when Grotrian and Edlén showed that the green line was emitted by a species of highly ionized iron, Fe XIV. This led to the discovery of another mystery. Since Fe XIV is an iron atom that has lost 13 of its 26 orbital electrons, such an ion can only exist in a very hot gas with a temperature of about $2 \mathrm{MK}$. This means that the corona is hundreds of times hotter than the surface of the Sun. As usually, the corona should be cool rapidly due to the loss of its heat by radiation and the solar wind. It is no doubt that additional energy of some form has to be pumped up from the Sun's surface, but what and how? There are many ideas to explain the extraordinary warmth of the Sun's corona. They could be dissipation of waves and/or electrical currents, which are induced by convective motions in the photosphere and magnetic activities. On the other hand, it has been found since long time that the properties of the fast solar wind cannot be adequately described by models based on classical heat conduction if not extended coronal heating in some form is provided.

It is clear that the heating of the solar corona and the origin and acceleration of the solar wind are among the important unsolved problems of space plasma and solar physics.

\subsection{Outline of the thesis}

In this thesis, the general properties of equatorial coronal holes and their relation to the high-speed solar wind streams will be investigated, by combining the remote sensing observations of the Sun (with SUMER, EIT, MDI on board SOHO and NSO/KP) as well as in situ observations of the solar wind (with SWE and MFI on board WIND). We expect that such coordinated observations can give us a clearer physical picture of the plasma conditions prevailing at the coronal hole base.

The thesis is organized as follows: A review of "coronal holes and origin of the fast solar wind" is given in Chapter 2; In Chapter 3, the instrumentation and diagnostic principles are described in details; Then we illustrate observations and general methods of data analysis in Chapter 4; The morphology of equatorial coronal holes are discussed in Chapter 5; In Chapter 6, we present results obtained from statistical and quantitative analysis; Equatorial coronal holes and their relation to the high-speed solar wind streams observed at 1 AU are studied in Chapter 7; Then, in Chapter 8, it is followed by a discussion of the implications of our observational results to coronal heating and solar wind acceleration; Finally, a brief summary is given in the last chapter. 


\section{Chapter 2}

\section{Review: Coronal Holes and Origin of the Fast Solar Wind}

\subsection{Overall description of coronal holes}

\subsubsection{Introduction}

Coronal holes are regions with significantly reduced emission in all spectral lines formed at coronal temperatures (at a lower temperature, coronal holes are also distinguishable if seen in the He I and He II emission), which can last over long period of time. Physically, they are known as regions of a low plasma density and a low electron temperature and of open magnetic fields. In the large-scale open fields, which are rooted on the Sun's surface and expand from there divergently outward into the interplanetary space, the plasma can be strongly accelerated, becomes supersonic, and forms the fast solar wind.

The earliest discovery of coronal holes outside the polar caps may be dated back to the 1950's. By analysing ground-based monochromatic images of the Fe XIV line intensity (5303 Å, green coronal line) observed in the 1940's, Waldmeier (1957, 1975) recognized that regions with very low emission could be identified on several consecutive rotations and called them 'Löcher', which mean holes in German. Since the 1960's, with the beginning of space-based observations, coronal holes have been studied intensively. In the
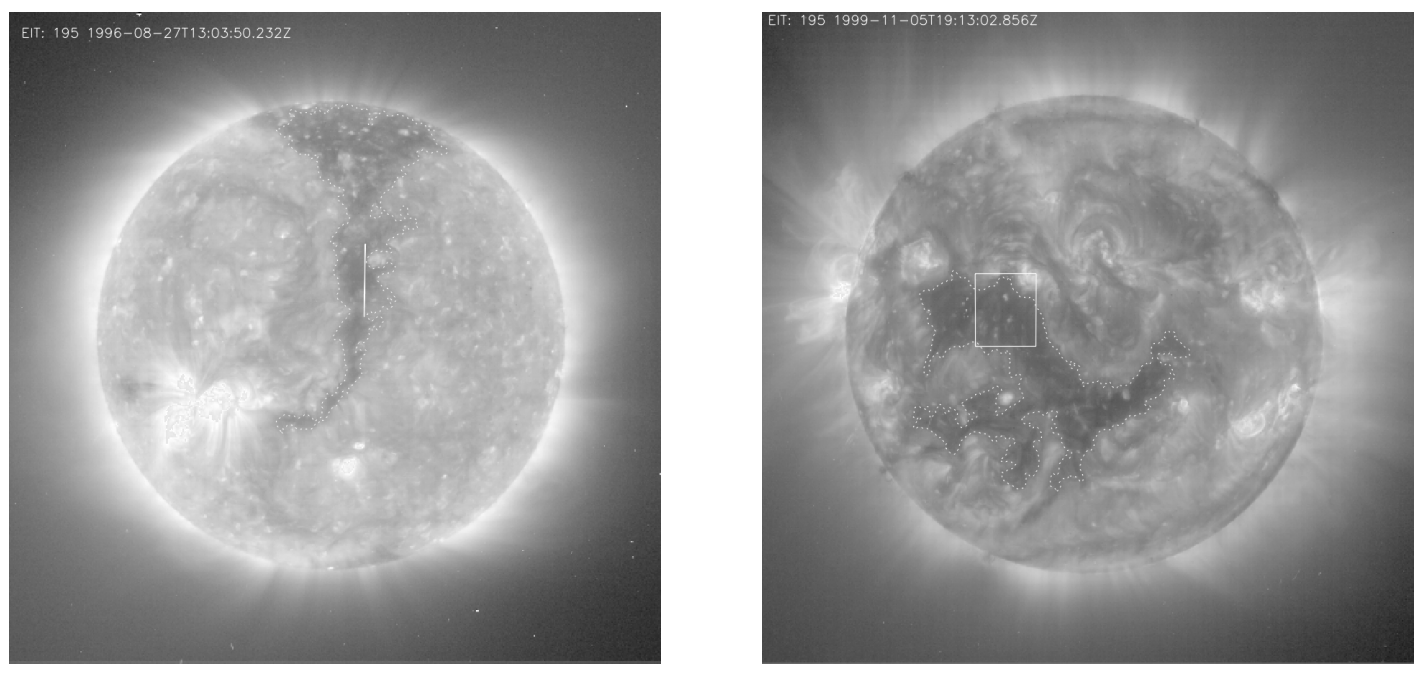

Figure 2.1: The EIT images show different types of the coronal hole. Left: north polar coronal hole and its equatorward extension resembling an "Elephant's trunk" (1996); Right: isolated equatorial coronal hole (1999). 
early 1970's, they were identified as the source of the fast solar wind by observational evidence (e.g., Krieger et al., 1973) as well as by theoretical arguments (Noci, 1973).

As a general classification, coronal holes may roughly be grouped into two categories. First, during the solar minimum, two persistent large coronal holes of opposite magnetic polarity cover each pole of the Sun, which are named polar coronal holes (PCHs) and are associated with the polar fields. This was one of the earliest discoveries from Skylab (1973-1974) (Bohlin, 1977a). Second, smaller coronal holes are frequently present as isolated features at lower latitudes around the maximum of the solar cycle. If such holes occurred near the Sun's equator, we usually define them as equatorial coronal holes (ECHs). In addition, during the declining phase of the solar cycle, the polar coronal hole may occasionally extend from the polar cap to the equatorial region, which is an example shown in Figure 2.1. Here we classify such a hole as an equatorial coronal hole.

\subsubsection{Coronal holes seen in various wavelengths}

Coronal holes can be distinguished from other structures in a wide range of spectra, including the radio, near infrared, visible light, ultraviolet and X-ray wavelengths. This has been found by the time of Skylab (Bohlin, 1977b).

Since the early 1960's, many soft X-ray (2-60 A) images have been obtained from instruments onboard rockets or space-crafts. The soft X-rays are formed at coronal temperatures of several million degrees, and thus can be observed directly against the cooler solar disk. In soft X-rays, coronal holes appear as dark regions with sharply reduced emission. The intensity, for example, observed by AS\&E sounding rocket, was found to be lower by a factor of about $1 / 3$ for the coronal hole than for the adjacent large-scale closed-loop structures (Krieger et al. 1973). The boundaries of the holes defined by the soft X-rays are rather complicated. On the one hand, foreground coronal emission may frequently hide a coronal hole or give a systematic displacement of its boundaries from their true surface locations for a hole far from the disk center (Bohlin, 1977b). On the other hand, a magnetic interaction near the boundaries may result in a more diffuse boundary of the coronal hole, as was found by recent observations of the Yohkoh Soft X-ray Telescope (Kahler and Hudson, 2002).

Ultraviolet emission lines and continua have a wide range of formation temperatures from about $10^{4} \mathrm{~K}$ to several $\mathrm{MK}$, which are emitted from the chromosphere, transition region and corona of the solar atmosphere, respectively. Therefore, they can also be observed directly against the solar disk. Advantages of using FUV/EUV to probe the upper solar atmosphere are that they can be observed with detailed spatial and spectral resolutions, so that detailed spatial structures and line shapes can be analyzed. Like the X-rays, EUV lines formed at coronal temperatures generally have greatly depressed emissions in coronal holes. Seen in these lines, the network structure is nearly totally indistinguishable from the cell interiors. With the exception of He I, He II and their continua which behave also like coronal lines, other chromospheric lines show a less pronounced effect in the coronal 
holes when compared with quiet regions (see, e.g., Huber et al., 1974; Withbroe, 1977). Such behaviours of the coronal holes can be seen elsewhere in the EIT full-disk images.

The radio emission has been assumed to be produced by thermal bremsstrahlung in the quiet Sun and the coronal hole. Although radio observations have the disadvantage of a low angular resolution, and easily suffer from refraction and scattering by irregularities in the atmosphere, they can be used to probe the solar atmosphere spanning from the lower chromosphere to the outer corona by estimating the density and temperature (see, e.g., Papaiannis and Baker, 1982). The first radio observations of coronal holes were reported by Dulk and Sheridan (1974). Seen around the metric wavelength, a pronounced decrease of the radio brightness could be found inside coronal holes compared with that in the quiet Sun. Spatially, there is a close relationship between the dark regions seen on radio maps and the coronal holes identified by EUV, X-ray and white-light observations (Dulk and Sheridan, 1974; Papaiannis and Baker, 1982).

In visible wavelengths, the photospheric emission is too bright on the disk so that coronal emission is impossible to detect. However, corona holes above the limb can be observed as very dark features during eclipses or by coronagraphs and K-coronameters. Observations by coronagraphs at the Fe XIV line ( $5303 \AA$ ) led to the earliest attention of the coronal holes on the disk (Waldmeier, 1957). Above the limb, the result has been confirmed again by recent observations of LASCO C1 on SOHO (Schwenn et al., 1997).

The well known absorption line formed by $\mathrm{He} \mathrm{I}$ in a near infrared wavelength at 10830 $\AA$ shows an apparent signature in coronal holes (Harvey et al., 1975). It has a greatly reduced absorption in coronal holes. This line is very useful since it can be observed from the ground.

\subsubsection{The morphology of coronal holes}

\section{Occurrence, size and distribution}

The polar coronal holes are persistent features during the solar minimum, shrink slowly with the increase of the solar activity, and are generally absent at the maximum phase (Broussard et al., 1978). They can extend down to a latitude of $60^{\circ}$ in each hemisphere (Wang et al., 1996). During the Skylab mission (June 1973-January 1974), it was found that the polar holes occupied about 15\% of the total area of the Sun (Bohlin, 1977a). Their area decreases with the increase of sunspot numbers (see, e.g., Broussard et al., 1978; Dorotovic, 1996; Bravo and Stewart, 1997). However, the number of non-polar holes and their distribution on the solar disk changed significantly with the solar cycle (Broussard et al., 1978; Insley et al., 1995; Belenko, 2001; Sanchez-Ibarra, 1990). They are very few and are confined to a narrow band near the equator at the solar minimum. At the solar maximum, smaller non-polar holes dominate at mid-latitudes, but with a large spread in latitudes. Such holes have a distribution on the disk very similar to a butterfly diagram, if it is plotted versus the solar cycle. The area occupied by them was found to be from about $1 \%$ to $5 \%$ of the total area of the Sun during Skylab mission(Bohlin, 1977a). 


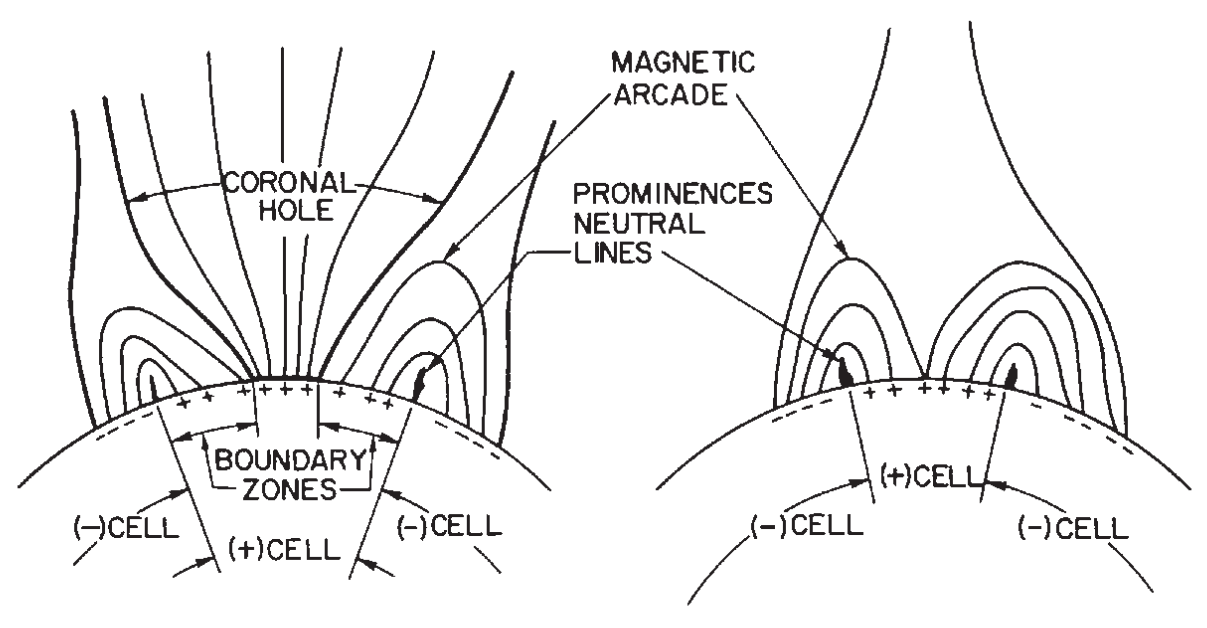

(a)

(b)

Figure 2.2: (a) Outline of the magnetic field configuration in which a coronal hole occurs; (b) A coronal hole may be absent if an unipolar magnetic region has a small size $\left(\leq 30^{\circ}\right)$. After Bohlin and Sheeley (1978)

\section{Formation and evolution}

It has been found that the existence of the coronal holes is directly associated with the disk activity. An "unbalanced flux" model was suggested to be responsible for the formation of a coronal hole (Timothy et al., 1975; Bohlin and Sheeley, 1978). The key model assumptions can be described as follows. Two or more bipolar magnetic regions (BMRs) are sufficiently close together to form a unipolar magnetic region (UMR), which can not be connected back to the Sun's surface (the flux becomes unbalanced). A coronal hole then occurs if this unipolar magnetic region is large enough (see Figure 2.2p), while a coronal hole may be absent if the size of this UMR is insufficient (see Figure 2.2p). After the formation of a coronal hole, it can remain nearly unchanged in area between successive rotations. Very often holes are either growing or decaying at a typical rate of $1.5 \times 10^{4}$ $\mathrm{km}^{2} \mathrm{~s}^{-1}$ (Bohlin, 1977a). During Skylab, this rate was true for the polar holes as well as lower-latitude holes. The lifetimes of non-polar coronal holes were found to range from one solar rotation to more than 10 solar rotations, while the polar holes are permanent features during the solar minimum. However, the physical mechanisms for the formation and maintenance of coronal holes are not yet well understood.

Previous studies of the rotational characteristics found that holes, which extend from the poles to the equatorial region, tend to rotate nearly rigidly (see, e.g., Timothy et al., 1975; Wagner, 1975). This has been confirmed later by many authors (see recent work, Kahler and Hudson, 2002). However, the isolated holes may exhibit significant differential rotation, but still rotate more rigidly than the photosphere (Insley et al., 1995). Wang et al. (1996) suggested that the rigid rotation of the polar hole extensions is caused by the in- 
teraction between the bipolar magnetic region (BMR) and the background dipole field. In their explanation, the coronal hole has dynamic boundaries, where field-line reconnections continually take place in the corona, thus the coronal field configuration rearranges itself instantaneously in response to the differential motion of the photosphere. This process keeps the hole rotating nearly rigidly. Moreover, it is worth noting that a rigidly rotating corona may also be a general feature for the normal solar corona. Recently, new observations by LASCO $\mathrm{C} 1$ coronagraph on $\mathrm{SOHO}$ have shown a general rigidly rotating Fe XIV (green coronal line) corona with a period of about 27.5 days at solar minimum, without significant deviation with latitude or with distance from the Sun (Stenborg et al., 1999, Inhester et al., 1999).

\subsubsection{Underlying photospheric magnetic fields}

The field strengths for holes have been measured by various authors (see, e.g., Levine, 1977; Bohlin and Sheeley, 1978; Harvey et al., 1982; Belenko, 2001). Following the studies by them, the average net flux density (field strength) in a coronal hole is signed that means the hole is predominantly unipolar. For holes at low and middle latitudes, where the photospheric magnetic field can be measured reliably, average field strengths were found to vary with solar cycle, ranging from 3 to $36 \mathrm{G}$ near the solar maximum and from 1 to $7 \mathrm{G}$ near the minimum during 1975-1980 (Harvey et al., 1982). The total magnetic flux for these holes was estimated to be about $2-5 \times 10^{21} \mathrm{Mx}$ near the minimum and $10-$ $15 \times 10^{21} \mathrm{Mx}$ near the maximum. For polar coronal holes, measurements of the magnetic field are difficult due to the line-of-sight geometry. By indirect measurements through the number of faculae, the total flux in polar coronal holes was estimated to be about $10 \times 10^{21} \mathrm{Mx}$ (Sheeley, 1976). If this estimated value was accepted, Harvey et al. (1982) noted that the decrease of the magnetic flux in polar holes was roughly compensated by the increase of the flux in low-latitude holes during 1975-1980. On the other hand, the average magnetic field strength was previously estimated to range from "no more than a few Gauss" (Howard and LaBonte, 1981) to "6 G above 55 latitude" (Svalgaard et al., 1978). Recently, interplanetary magnetic fields above the polar hole have been measured during the Ulysses mission, and the average field strength of the radial component was deduced to be about $3.1 \mathrm{nT}$ at $1 \mathrm{AU}$ (Forsyth et al., 1996). Thus the magnetic strength at the base of these polar holes can be estimated to be about $10 \mathrm{G}$ by magnetic flux conservation, if an expansion factor of 7 is assumed for these holes. This estimation is also consistent with the modelling work by Banaszkiewucz et al. (1998), who used a simple analytic model for the coronal magnetic field to describe solar minimum conditions.

While the magnetic field in a coronal hole is dominated by a single polarity, a significant amount of the magnetic flux with opposite polarity also exists. Levine (1977) suggested that this portion of the flux must be locally closed. Observations of the photospheric magnetic field suggested that the flux of this opposite polarity occupies about $10 \%$ in coronal holes (see Wilhelm, 2000, and references therein). However, this ratio may vary in different coronal holes. For example, a ratio of about $30 \%$ has been measured in the coronal 
holes observed in 1996 (Belenko, 2001). According to the FUV observation, Wilhelm (2000) also argued that the expected ratio would likely be much higher than a value of $10 \%$.

\subsubsection{Fine structures in coronal holes}

As viewed in a large scale, coronal holes are much more homogeneous when observed at coronal lines in ultraviolet and X-ray wavelengths in comparison to other regions of the Sun. However, if they are seen in chromospheric and transition region lines, the network is the most basic and obvious structure. Moreover, coronal holes appear highly structured at any time if they are observed with high spatial and temporal resolution. Such structures include polar plumes, bright points, spicules, macrospicules, explosive events and so on. The scales of these structures in coronal holes spatially range from 1 arcsec to several tens of arcsec, and temporally from 1 minute to several days. Because they may be very important to understand the basic processes of coronal heating and solar wind acceleration, numerous efforts have been made since the beginning of high-resolution observations. We give here a brief description of them.

\section{Network}

The chromospheric network is the most common feature in both coronal holes and quiet regions. It appears as white or dark patches seen in spectroheliograms observed in the chromosphere and low transition region. From photospheric magnetograms, the network is identified as regions with clusters of magnetic flux fibers that are suggested to have small sizes of less than $1^{\prime \prime}$ and field strengths of $1-2 \mathrm{kG}$ (see, e.g., Zwaan, 1987, and references therein). Over a time scale of hours to a day, its location and magnetic flux are relatively steady, although the shape of some clusters changes apparently. The network has been found to correspond to the boundaries of supergranulation cells that have a typical diameter of about $3 \times 10^{5} \mathrm{~km}$. The width of the network is about $10^{\prime \prime}$ (Reeves, 1976; Patsourakos et al., 1999) in the chromosphere and transition region. The area occupied by the network is estimated to be $10 \%$ in the upper photosphere. For the transition region, it was found that the network occupies $40 \%-50 \%$ of the area and contributes about $70 \%$ of the total emission (Reeves, 1976, Gallagher et al., 1998).

In the network, the magnetic pressure dominates the thermal pressure, the magnetic fields are firstly constrained at the boundaries of supergranular cells, created by the convective motion of the photospheric material, but then expand quickly with height from the transition region into the lower corona and attain a funnel shape due to the changing equilibrium between the magnetic and thermal pressure in the gravitationally stratified solar atmosphere. Under the assumption of a static atmosphere, Gabriel (1976) constructed the first network model (see Figure 2.3). This model interprets the enhanced emission in the network as the main result of reduced temperature gradient there. Instead of Gabriel's 


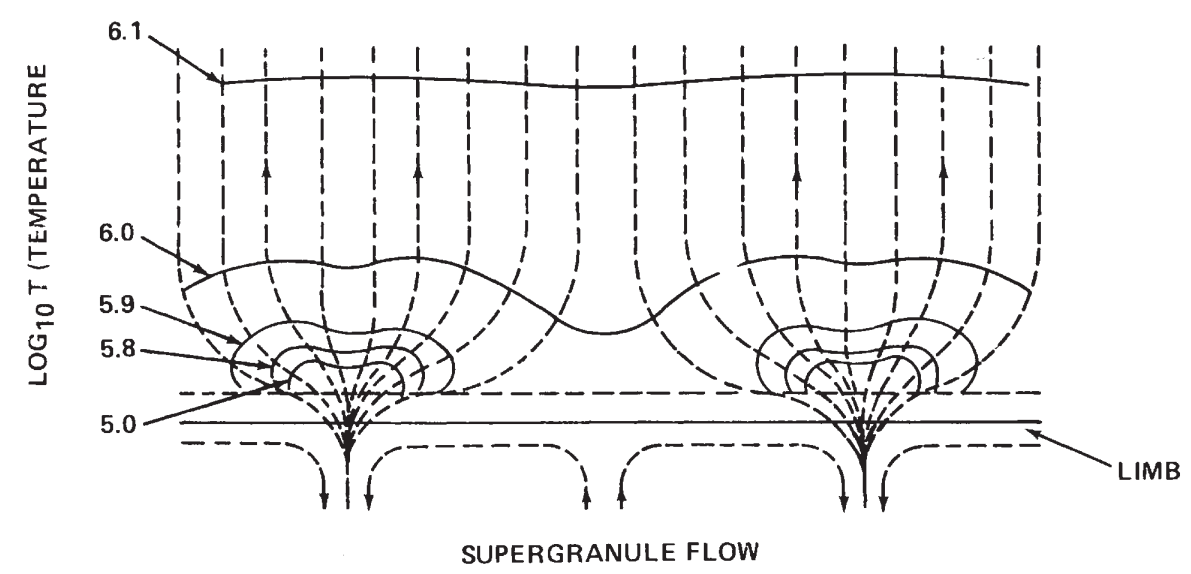

Figure 2.3: Gabriel's model: magnetic configuration and temperature contours. After Gabriel (1976)

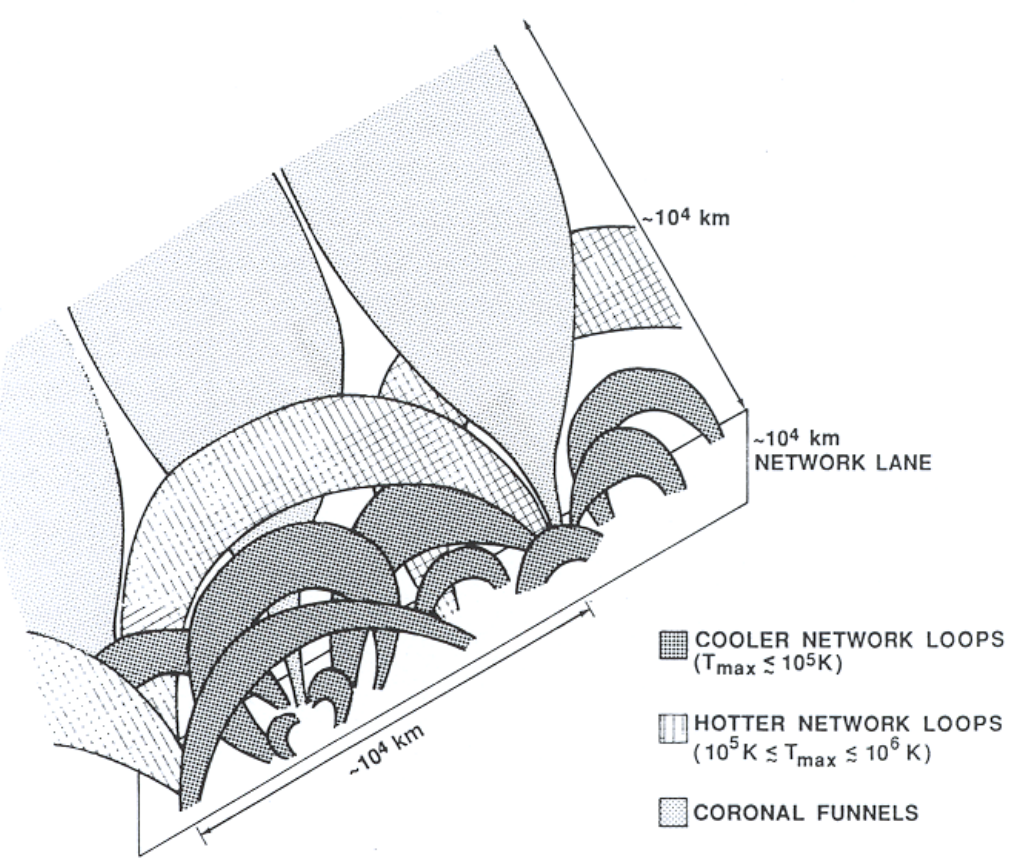

Figure 2.4: Dowdy's model: magnetic structures in the network lane. A 'magnetic junkyard' is collected into the network lanes by supergranulation flow. After Mariska (1992)

conduction driven model, a flow driven model can also attain a similar magnetic configuration (Pneuman and Kopp, 1978). Based on the fact that many fine-scale structures with mixed polarities are present in the photospheric magnetic network, Dowdy et al. (1986) suggested a modified model in which only a fraction of the network flux opens into the corona as a funnel shape. The rest of the network is occupied by a population of low-lying loops with lengths less than $10^{4} \mathrm{~km}$ (see Figure 2.4). 
In the coronal hole, the properties of the network are very similar to the quiet Sun, if observed below the middle transition region. Above the upper transition region, the network appears more hazy or nearly absent. The network has been suggested as the ultimate source of the solar wind by both theoretical ideas (Axford and McKenzie, 1992, 1997; Marsch and Tu, 1997) and observations (Hassler et al., 1999). This will be discussed in Sections 2.4.2 and 2.5.2.

\section{Bright points}

Bright points (BPs) are the common events in both coronal holes and quiet Sun regions. They were first observed in soft X-ray images and called X-ray bright points (XBPs) (Vaiana et al., 1970). Actually, they are visible at X-ray, EUV and radio wavelengths with enhanced coronal emission, and often associated with plumes in polar coronal holes. Bright points have a typical size of about $10^{\prime \prime}-40^{\prime \prime}$, often with a $5^{\prime \prime}-10^{\prime \prime}$ bright core; and a typical lifetime ranging from 2 to 48 hours (see, e.g., Golub et al., 1976, Habbal et al., 1990, Habbal, 1992). Bright points were found to appear on the magnetograms as bipolar features, except for very newly emerged or old and decayed ones. The total magnetic flux in a typical bright point living about 8 hours was estimated to be about $2 \times 10^{19} \mathrm{Mx}$ (Golub et al., 1977). In the cooler transition-region lines, bright points are spatially coinciding with network boundaries, but they are not easily distinguishable from the rest part of the network, since they all have enhanced emission. Furthermore, the properties of bright points are very similar in both $\mathrm{CH}$ and QS regions (Esser and Habbal, 1997). Non-thermal velocities derived from the C IV line width were found to be only marginally larger inside the $\mathrm{CH}$ than in the QS region (Dere et al., 1989b).

\section{Plumes}

Plumes are ray-like structures above the polar caps. They were first found in white light as polar rays (see, e.g., Saito, 1965; Koutchmy, 1977). Since the Skylab time, they were also investigated in EUV wavelengths (see, e.g., Bohlin et al., 1975) and in soft X-rays (see, e.g., Ahmad and Webb, 1978). The typical plumes have a lifetime of several hours to days. At the base of plumes, bright points are observed in EUV, soft X-rays and radio emission. Plumes are estimated to occupy about $10 \%$ of the polar hole volume and reveal superradial expansion to 30 solar radius, if seen in white light (Deforest et al., 2001). Various efforts have been made to measure the temperature and density of plumes at the base and above the limb (see, e.g., Ahmad and Withbroe, 1977; Walker et al., 1993; Deforest et al., 1997; Wilhelm et al., 1998b; Young et al., 1999). From these studies, plumes were identified as cooler and denser structures as compared to the surrounding atmosphere in coronal holes.

One question to be answered is what are the magnetic structures that plumes are associated with at the base. Some observations have shown that plumes are rooted in unipolar regions (see, e.g., Harvey, 1965; Newkirk and Harvey, 1968; Deforest et al., 1997). Deforest et al. (1997) reported observations from MDI/SOHO and CDS/SOHO data and 
showed that plumes are located over complex unipolar magnetic flux concentrations (with field strengths of about $100 \mathrm{G}$ at the photosphere) on the boundaries of chromospheric network cells. However, many people suggested that plumes are associated with mix-polarity magnetic features at the footpoints (see, e.g., Ahmad and Withbroe, 1977; Habbal, 1992; Wang and Sheeley, 1995; Wang et al., 1997; Wang, 1998), and formed by magnetic interaction between bipoles and background unipolar fields. Wang et al. (1997) argued that the previous conclusion by Deforest et al. (1997) may result from the loss of most of the minority flux. This loss is caused by a cutoff below $\left|B_{\text {los }}\right| \sim 20 \mathrm{G}$ on the line-of-sight polar magnetic fields in their study.

Another important question concerning plumes and interplume regions in polar coronal holes is what a role they play on the origin of the fast solar wind. While some authors suggested that plumes can provide nearly all the mass flux for the fast solar wind (see, e.g., Ahmad and Webb, 1978; Mullan and Ahmad, 1982; Walker et al., 1993), others show that they can not be the main source, based on modelling calculations (Wang, 1994) and Doppler shift measurements (Hassler et al., 1997; Wilhelm et al., 1998b; Wilhelm, 1999, Wilhelm et al., 2000).

\section{Spicules}

Spicules are traditionally seen on the limb in the $\mathrm{H}_{\alpha}$ line. They are jet-like structures (with sharp boundaries that extend from the chromosphere upward into the corona) with a velocity of about $25 \mathrm{~km} \mathrm{~s}^{-1}$. Their bases lie 1000 to $2000 \mathrm{~km}$ above the photosphere and their roots disconnect from the surface. After having reached their maximum of height, spicules are found either falling back into the chromosphere or fading out in chromospheric spectral lines. Typically, spicules have lengths ranging from 5000 to $10000 \mathrm{~km}$, widths from several hundred to $2000 \mathrm{~km}$ and lifetimes from 1 to 10 minutes, respectively. They are estimated to cover $1-2 \%$ of the Sun's surface. The temperature of spicules is inferred to be around $10^{4} \mathrm{~K}$, similar to that of the upper chromosphere. Their typical density is measured to be approximately $10^{11} \mathrm{~cm}^{-3}$.

On the solar disk, spicules are not yet well identified perhaps due to being either too small or too transparent (Kneer, 1992). However, many authors identify them as dark and bright mottles because most of their physical parameters are very similar to those of spicules, except for the relatively lower velocity in mottles (Grossmann-Doerth and Schmidt, 1992; Tsiropoula et al., 1993). They appear to cluster together as "bushes" (near the limb) or "rosettes" (on the disk) around the supergranular cell boundaries, and occur rarely in active regions where the magnetic fields are much stronger. Spicules are reported to be slightly taller and oriented more nearly vertical in the polar coronal holes, and somewhat more numerous in polar latitudes than in low latitudes. However, the exact physical conditions of spicules are not yet well defined from observations because of their small sizes.

Spicules can also be observed above the limb in EUV lines. According to the SUMER observations (Budnik et al., 1998; Wilhelm et al., 2000; Wilhelm, 2000), EUV spicules 
have a width of $10^{\prime \prime}-20^{\prime \prime}$ when seen in transition region lines, and disappear at a temperature above 0.5 MK. Spicules and macrospicules have also different orientations observed in $\mathrm{CH}$ regions (see the figures in Wilhelm, 2000; Wilhelm et al., 2000). The connection between the $\mathrm{H}_{\alpha}$ and EUV spicules has not yet established. However, it is suggested that the EUV spicules are very likely a hot sheath of the cooler $\mathrm{H}_{\alpha}$ spicules (Sterling, 2000).

The detailed properties of spicules have been reviewed by many authors (see, e.g., Beckers, 1968, 1972; Sterling, 2000; Wilhelm, 2000, and references therein). It is believed that spicules originate from the magnetic network. Some mechanisms for generating spicules have been suggested, for example, convective instabilities, magnetic reconnections, shock waves and Alfvén waves. The mass and energy flow carried by spicules may play a very important role on the overall mass and energy balance of the chromosphere and corona (Withbroe and Noyes, 1977). Averaged over the Sun's surface, spicules carry an upward mass flux of about $4 \times 10^{-9} \mathrm{~g} \mathrm{~cm}^{-2} \mathrm{~s}^{-1}$ into the corona, if $1 \%$ areal coverage of spicules is considered. Compared with that, the mass flux taken away by the solar wind is about $3 \times 10^{-11} \mathrm{~g} \mathrm{~cm}^{-2} \mathrm{~s}^{-1}$. Because the portion taken away by the solar wind is only $1 \%$, most of the spicular material must fall back into the chromosphere. It has been suggested that this falling material may likely be responsible for the systematic redshifts measured in transition region lines. This idea is consistent with the fact that the globally averaged downward hydrogen flux inferred from UV observations is comparable to the upward hydrogen flux in spicules (Withbroe, 1983).

\section{Explosive events}

Explosive events were first observed by NRL/HRTS instrument (Brueckner and Bartoe, 1983). They occur very frequently (a global birthrate of 600 events per second), with a small spatial scale of $1500 \mathrm{~km}$, a short lifetime of $60 \mathrm{~s}$ and a mean energy of $10^{24} \mathrm{ergs}$ per event. They can be seen in spectral lines with a formation temperature ranging from $2 \times 10^{4}-5 \times 10^{5} \mathrm{~K}$, with line profiles shown as highly broadened and/or shifted in both red and blue wings (non-Gaussian shape) (Brueckner and Bartoe, 1983; Dere et al., 1984, 1989a; Innes et al., 1997a). Explosive events tend to occur throughout the quiet-Sun network, where mixed-polarity magnetic features are present (Chae et al., 1998b). Dere et al. (1991) suggested that explosive events are formed by fast magnetic reconnection. Observations with SUMER showed that explosive events are not visible in emission lines of Si II, C I and O I, which are formed at a low temperature in the chromosphere. Moreover, explosive events produce apparently bi-directional flows with high velocities. This imply that they are indeed associated with magnetic reconnection (Innes et al., 1997a b). Recently, Winebarger et al. (2002) analyzed the energetics of explosive events observed by SUMER and found that these events globally release about $4 \times 10^{4} \mathrm{ergs} \mathrm{cm}^{-2} \mathrm{~s}^{-1}$ of energy flux, which indicates that they may not be significant for heating the solar atmosphere directly. However, their power-law spectral index for the energy may imply that there are currently undetectable events with a lower energy of about $10^{22}$ ergs, which would release enough energy to heat the solar atmosphere. 


\subsection{FUV/EUV observations of coronal holes}

Since the beginning of the 1970's, numerous observations with high spatial and spectral resolution in FUV/EUV have been carried out onboard spacecrafts and rockets. This has provided us with powerful means to probe the solar atmosphere. FUV/EUV spectral analysis can be used to derive plasma parameters, such as the electron density, temperature, bulk and non-thermal velocity. These parameters are very crucial for a good understanding of the detailed physical processes occurring in coronal holes, as well as for the modelling work. In the following some results from previous studies, in particular, recent results during SOHO mission, will be reviewed. The diagnostic methods will be discussed in Chapter 3.

\subsubsection{Observations of FUV/EUV radiance}

During the Skylab mission, Huber et al. (1974) analyzed the spectroheliograms obtained by Apollo Telescope Mount (ATM) near the center of the solar disk. The lines analyzed by them have formation temperatures ranging from $10^{4} \mathrm{~K}$ to $1 \mathrm{MK}$. Although the hole boundary was obvious only in Mg X, Ne VIII and Ne VII lines, a more thorough study of these spectroheliograms showed that all lines had weakened network boundaries within the coronal hole. Their data showed further that intensities of various lines were more uniform and weaker inside the hole than outside, depending on the formation temperatures. This trend is consistent with previous observations with the Harvard College Observatory (HCO) on OSO-4 (Munro and Withbroe, 1972). The intensity decrease of Mg X (625 $\AA$ ) is found to be a factor of about 5 , and the network structure seen in this line appears totally absent. Lines formed in the chromosphere and transition region have an intensity reduction of about $15-30 \%$ for the cell area, and $30-35 \%$ for the network. However, using the same instrument, Reeves (1976) found that intensities of the transition region lines have no obvious difference in the cell interiors between holes and quiet regions. Furthermore, measurements of the emission of various ions above a polar coronal hole showed an increase of the limb height in the holes, which gave a quantitative indication of the increased thickness of the transition region underlying coronal holes (Huber et al., 1974). This effect is particularly apparent for the Ne VII line (465 $\AA$ ), confirming the previous report by Tousey et al. (1973). These results led to the conclusion that the thickness of the transition region increases by a factor of about 5-10 (Munro and Withbroe, 1972; Huber et al., 1974). On the other hand, Doschek et al. (1975) argued that the apparent increase of the limb height may have been contributed by inhomogeneities (e.g., spicules).

During the SOHO mission, SUMER, CDS and EIT have been used to observe both polar holes and equatorial holes on the disk and/or above the limb. Coronal holes usually appear as dark features in the EIT full-disk images obtained in its coronal channels. The EIT fulldisk images are very useful for tracing the coronal holes, and can also be used to study the behaviours of the holes at a high temperature above $1 \mathrm{MK}$. 
In polar coronal holes, Stucki et al. $(2000 \mathrm{~b}, 2002)$ analyzed a large number of lines observed by SUMER and CDS. They found that the variations of average intensities between holes and quiet regions are generally in agreement with a previous study by Huber et al. (1974), except for the Ne VII line observed by CDS, which seems to be unaffected by the hole. Del Zanna and Bromage (1999) studied the "Elephant's Trunk" coronal hole occurring in 1996 using data obtained by CDS. Again, their data showed that the network seen in coronal lines is absent in the hole. Lemaire et al. (1999) reported a small coronal hole near the equator, observed by SUMER in several transition region lines in 1997. These lines have formation temperatures in the range of $1 \times 10^{5} \mathrm{~K}$ to $6.3 \times 10^{5} \mathrm{~K}$ (N III, N IV, S $\mathrm{V}, \mathrm{O}$ IV, O V and Ne VIII). They found that the average intensity of transition region lines is lower by $30 \%-40 \%$ in the coronal hole than in the quiet regions. Wilhelm et al. (2002) have analyzed several equatorial coronal holes observed by SUMER in 1999. They found that the characteristics of equatorial coronal holes are very similar to those of polar coronal holes observed during the sunspot minimum. The radiance ratios decrease with the formation temperature from $\mathrm{L}(\mathrm{CH}) / \mathrm{L}(\mathrm{QS})=1$ in the continuum to about 0.3 for $\mathrm{Mg}$ IX. All coronal holes studied by them show no difference for the brightness and the structure of the chromospheric network seen in the continua and the Si II and O II lines.

A detailed morphological study of both coronal holes and quiet Sun has been recently carried out by Feldman et al. (2000b). Some of their results may be summarized as follows: For the lines at temperatures between $2 \times 10^{4}-6 \times 10^{5} \mathrm{~K}$, most of the emission comes from elongated loop-like structures. These structures have typical visible lengths of $10^{\prime \prime}-20^{\prime \prime}$, widths of $2^{\prime \prime}$ at most and lifetimes of 5-10 min. Among them, the brightest structures are rooted along the network boundaries with orientations mainly perpendicular to the boundaries, and appear very similar at largely different temperatures. In images taken in these lines (except for He I and He II), coronal holes are almost indistinguishable from quiet Sun regions.

\subsubsection{Underlying chromosphere and transition region}

From the discussions above, we may summarize that the morphological properties of the chromosphere and transition region in coronal holes are quite similar to the ones of the normal quiet Sun, although the transition region lines exhibit some decrease in their intensities. For the transition region, the main difference between the quiet Sun and the coronal hole may be that coronal hole has a lower density, by a factor of about 2, and a higher limb extension than the quiet Sun (see, e.g., Munro and Withbroe, 1972; Huber et al. 1974). As we have mentioned above, this led them to propose that the temperature gradients in the transition region are somewhat lower in coronal holes than the quiet Sun, and consequently, that the pressure and the conductive flux may be reduced in coronal holes.

For the chromosphere in coronal holes, it should be mentioned that some authors also found a pronounced enhancement of the brightness relative to the quiet Sun in various 
chromospheric emission lines (Ly $\alpha$, Ly $\beta$, Ca II and Mg II, which are formed at temperatures less than 20,000 K) (Gopalswamy et al., 1999), as well as in microwaves at frequencies between 15 and $39 \mathrm{GHz}$ (Bocchialini and Vial, 1996).

\subsection{Plasma parameters deduced from observations}

\subsubsection{Plasma velocity inferred by Doppler shifts}

Given a sufficient spectral resolution, Doppler shifts can well be measured by determining the line central position. In a statistical sense, Doppler shifts are caused by the bulk motions or wave motions of plasmas. Measurements of Doppler shifts are very important to understand the dynamical nature of the source regions of the fast solar wind - coronal holes. Since the 1970's, numerous authors have measured Doppler shifts in various lines. In particular, during the SOHO mission, measurements of Doppler shifts were widely studied in various regions of the Sun with the SUMER spectrometer. Because the spectra observed by SUMER contain many lines in a wide range of wavelengths and have a high spectral resolution, new interesting results have been found in both $\mathrm{CH}$ and QS regions.

\section{Upper transition region and coronal lines $\left(\mathbf{T}_{e}>5 \times 10^{5} \mathbf{K}\right)$}

During the Skylab mission, Doppler shifts of three coronal lines were measured in an equatorial coronal hole ${ }^{1}$ with a rocket-borne grazing-incidence stigmatic spectrograph covering the spectral region 200-700 (Cushman and Rense, 1976). They found that these lines are on average blueshifted relative to outside a CH, for Si XI (303 $\AA$ ) about 13 $\mathrm{km} \mathrm{s}^{-1}$, for $\mathrm{Mg} \mathrm{X}(610 \AA)$ about $12 \mathrm{~km} \mathrm{~s}^{-1}$, for Mg IX (368 $\AA$ ) about $14 \mathrm{~km} \mathrm{~s}^{-1}$. Rottman et al. (1982) measured the line Mg X (624 $\AA$ ) with the rocket-borne EUV spectrometer LASP, and reported that the maximal measured shift corresponds to a velocity (relative to outside an $\mathrm{ECH}$ ) of about $12 \mathrm{~km} \mathrm{~s}^{-1}$ in the $\mathrm{Mg} \mathrm{X}$ line. For the polar hole, the mean relative blueshift of this same line was about $8 \mathrm{~km} \mathrm{~s}^{-1}$ (Orrall et al., 1983).

During SOHO mission, the Ne VIII resonance line at $770 \AA$, because of its high brightness, was frequently used to study Doppler shifts in coronal holes. With the updated rest wavelength ${ }^{2}$, it was found that this line is averagely blueshifted in coronal holes as well as in quiet and active regions, but the blueshift is larger in coronal holes (Hassler et al.,

\footnotetext{
${ }^{1}$ Later, Cushman and Rense (1977) pointed out that this coronal hole was not a X-ray coronal hole at that time when they measured it, but it was indeed a region of open magnetic field and became a X-ray coronal hole (Skylab CH 4) on the next solar rotation .

${ }^{2}$ New rest wavelengths of the $\mathrm{Mg} \mathrm{X}$ line at $624 \AA$, the Ne VIII line at $770 \AA$ and the Fe XII line at $1240 \AA$ were deduced by Peter and Judge (1999); Dammasch et al. (1999c|b), which seem to be the most accurate values obtained by using SUMER data. The new wavelengths suggested by them are $624.965 \AA$ for $\mathrm{Mg} \mathrm{X}$, $770.428 \AA$ for Ne VIII and $1241.990 \AA$ for Fe XII, respectively. They differ from the old values $624.943 \AA$, $770.409 \AA$ and $1240.00 \AA$ by $0.019 \AA, 0.022 \AA$ and $-0.01 \AA$, respectively.
} 
1999; Peter and Judge, 1999; Dammasch et al., 1999c; Wilhelm et al., 2000, Teriaca et al. . 1999). The radial outflow velocity, derived from the line-of-sight Doppler shift, ranged from 5 to $12 \mathrm{~km} \mathrm{~s}^{-1}$ for polar coronal holes (Hassler et al., 1999; Dammasch et al. 1999c; Wilhelm et al., 2000). It should be mentioned that in these measurements an absolute wavelength calibration has been made, by using either cold lines as reference lines or assuming zero shifts above the solar limb.

In equatorial coronal holes, Wilhelm et al. (2002) reported outflow velocities of about 5 $\mathrm{km} \mathrm{s}^{-1}$ for the Ne VIII line and $15 \mathrm{~km} \mathrm{~s}^{-1}$ for the Mg IX line (706 $\AA$ ) with respect to quiet regions.

\section{Transition region lines $\left(\mathbf{T}_{e}<5 \times 10^{5} \mathbf{K}\right)$}

In the quiet Sun, one of the most interesting discoveries is that the emission lines of the transition region are systematically redshifted (see, e.g., Brekke et al., 1997, and references therein). In Figure 2.5, all measured transition lines, with a formation temperature below $5.5 \times 10^{5} \mathrm{~K}$, show apparent redshifts of about 2 to $12 \mathrm{~km} \mathrm{~s}^{-1}$. For lines with a formation temperature above $5.5 \times 10^{5} \mathrm{~K}$ (e.g., Ne VIII, Mg X and Fe XII), the Doppler shifts measured during the SOHO mission are blue, which seems to be in contradiction to previous measurements. This difference is mainly due to the use of new rest wavelengths of these lines. In addition, the redshifts show apparently a temperature dependence, which have a peak velocity near $\log T=5.2$.

Interesting implications arise from these results. If the measured Doppler shift can be interpreted as a steady plasma flow, the persistent downflow would imply that plausible mechanisms should be given to interpret how this mass flux is injected/fed into the transition region. Several hypotheses have been proposed to explain this question: the return of spicular material (see, e.g., Pneuman and Kopp, 1978; Athay and Holzer, 1982; Athay, 1984; Cheng, 1992); siphon flows in coronal loops (Boris and Mariska, 1982; Mariska and Boris, 1983); asymmetric heating of coronal loops (McClymont and Craig, 1987; Mariska, 1988; McClymont, 1989; Spadaro et al., 1991); radiatively cooling condensations (Reale et al., 1996, 1997). On the other hand, some authors suggested that the persistent redshifts may be interpreted as effects of nanoflare-induced waves propagating through coronal loops (Hansteen, 1993; Wikst $\varnothing 1$ et al., 1997). However, this mysterious problem has not yet been solved well. Detailed comments on advantages and disadvantages of every theory can be found in Brekke et al. (1997).

Similar measurements have not yet been studied systematically in coronal holes. Up to now, observations show that some transition region lines are on average also redshifted, but tend to have smaller velocities than in quiet regions (Dere et al., 1989b; Doschek et al., 1976). More data need to be analyzed to study their behaviours in coronal holes, however.

With a rocket-borne spectrograph, Rottman et al. $(1981,1982)$ analyzed the O V line (629 $\AA$ ) in an ECH. They obtained an apparent average outflow of about $3 \mathrm{~km} \mathrm{~s}^{-1}$ relative to the 

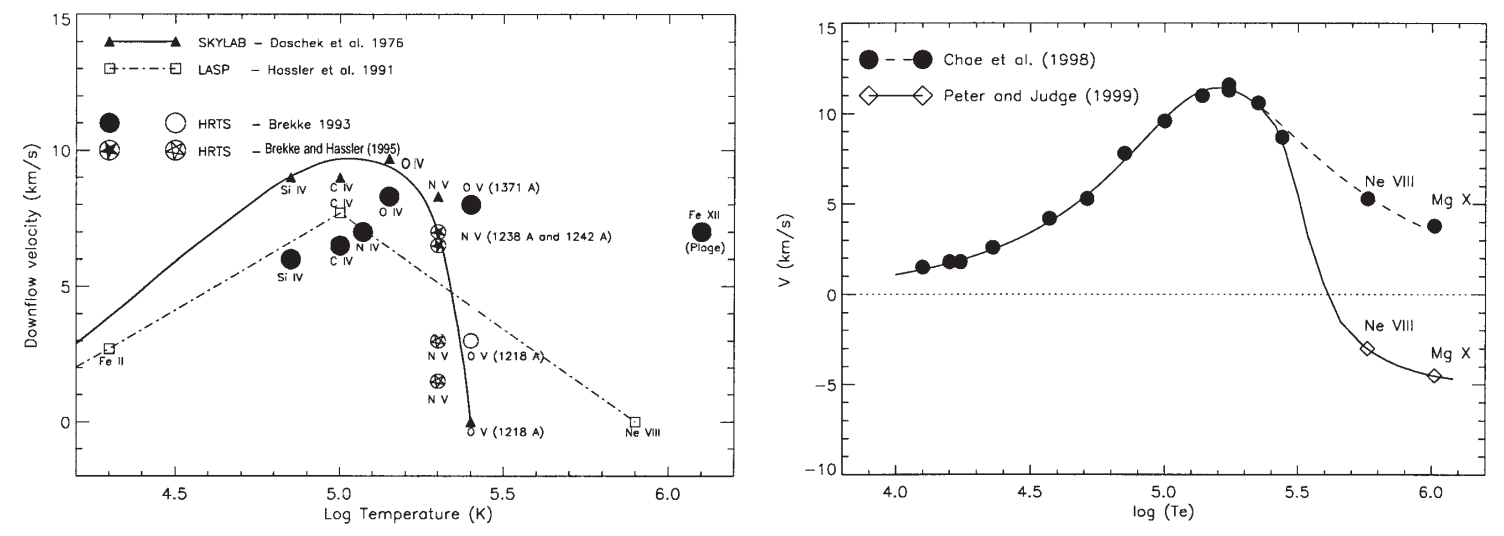

Figure 2.5: Observations of Doppler shift versus temperature in the quiet Sun. Left: measurements from pre-SOHO era; Right: measurements by SUMER during SOHO mission; After Brekke (1999)

quiet Sun, and the maximum shift corresponded to a velocity of about $7 \mathrm{~km} \mathrm{~s}^{-1}$. During the sixth rocket flight of NRL/HRTS (High Resolution Telescope and Spectrograph), Dere et al. (1989b) observed a coronal hole on the solar disk. With a nearly absolute calibration of the wavelength determined by the chromospheric line Si I, they obtained an average Doppler shift of the C IV resonance lines (1548 $\AA$ and $1550 \AA)$ that is a redshift of $2 \mathrm{~km}$ $\mathrm{s}^{-1}$ for the hole, and $10 \mathrm{~km} \mathrm{~s}^{-1}$ for the quiet region. Furthermore, they found that there is a larger proportion of outflowing plasma in the hole than in the quiet Sun. Inside the coronal hole $26 \%$ of the Doppler shifts are blueshifts, as compared with $7 \%$ in the quiet Sun. The average velocity in regions with blueshifts inside the hole is $5 \mathrm{~km} \mathrm{~s}^{-1}$.

During the SOHO mission, observations with SUMER and CDS were used to study Doppler shifts of transition region lines in polar coronal holes (Warren et al., 1997a; Peter and Judge, 1999; Stucki et al., 2000a b, 2002). Warren et al. (1997a) reported that the C II $(1037 \AA)$, N IV $(765 \AA)$ and O VI (1038 $\AA$ ) lines observed by SUMER are predominantly redshifted in the coronal hole. For N IV and O VI, however, there are more blueshifted profiles in the hole than in the quiet Sun. This latter tendency was also confirmed by Stucki et al. (2000b, 2002), who analyzed 26 spectral lines observed by SUMER and 14 lines by $\mathrm{CDS}$, and found that all lines formed above $10^{5} \mathrm{~K}$, as well as He I and He II lines formed below $10^{5} \mathrm{~K}$, are blueshifted inside the coronal hole compared to the normal quiet Sun. Moreover, this shift difference tends to increase with the temperature. Unfortunately, in the work by Stucki et al. (2000b, 2002), all Doppler shifts in the hole are compared to the quiet Sun, without absolute wavelength calibration.

In contrast to some authors (Dere et al., 1989b; Warren et al., 1997a), Peter and Judge (1999) and Peter (1999) reported that an evidence of a net outflow in polar holes was found in $\mathrm{C}$ IV and $\mathrm{O}$ VI lines. They suggested that this difference may result from the different filling factor of blueshifts between the $\mathrm{ECH}$ and $\mathrm{PCH}$, because they found $67 \%$ of the area in PCH is filled by blueshifts.

Net blueshifts were also measured in polar holes with a radial outflow velocity of about 
$8 \mathrm{~km} \mathrm{~s}^{-1}$ for the He I absorption line (10830 ̊) (Dupree et al., 1996), and with a line of sight velocity of about $3 \mathrm{~km} \mathrm{~s}^{-1}$ for the He I emission line (584 $\AA$ ) (Wilhelm et al., 2000).

\section{Doppler shifts related to line intensity and chromospheric network}

As we have discussed in Section 2.1.5, there are various structures within the coronal hole. Such structures can be distinguished by emission brightness or magnetic flux. One important question to be answered is what a role they play in the origin of the fast solar wind. Studying the relationships between Doppler shifts and line intensities may be used to probe the nature of the source regions of the fast solar wind.

In a low-latitude coronal hole, the Solar Maximum Mission (SMM) satellite has been used to observe Dopplergrams of the C IV line (Mullan and Waldron, 1987). In contrast with the results observed in other regions on the Sun (Gebbie et al., 1981; Athay et al., 1983a b; Peter, 1999), they found that scatter plots of C IV emission intensity versus velocity do not show a pronounced positive correlation. This has been confirmed also by Peter (1999).

In a polar coronal hole during the solar minimum, the relationship between the Doppler shift of the Ne VIII line (770 $\AA$ ) and chromospheric network has been studied by Hassler et al. (1999). They found strong evidence that the largest outflow velocities are closely as-

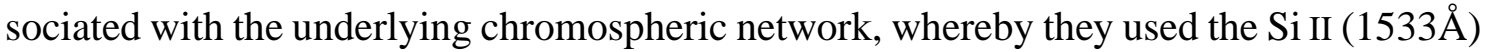
brightenings in the radiance image as an indication of the chromospheric magnetic network. An extended work, done by Stucki et al. (2000a), who did a statistical analysis, showed a positive correlation between the blue shift of the Ne VIII line and the intensity of the N IV line (765 $\AA$ ) (used also as an indicator of the network) in the polar coronal hole, and thus corroborated the results of Hassler et al. (1999).

Another interesting observational result is that the largest blue shifts of the Ne VIII line coincide spatially with very dark regions in the intensity image of the coronal hole when seen in the same line (Wilhelm et al., 2000). Bright points and polar plumes seen in $\mathrm{Ne}$ VIII do, however, not show signatures of outflow.

\subsubsection{Non-thermal velocity}

The non-thermal velocity is deduced from the measured Doppler width after subtraction of the portion contributed by instrumental broadening and thermal broadening (see Eq. 3.31 in Section 3.4.3). Previous measurements of the line width showed that spectral lines emitted by an optically thin plasma in the solar atmosphere are generally broadened in excess of their thermal width, under the assumption that they are formed in ionization equilibrium, and that the ion temperature equals to the electron temperature. It is suggested that this extra broadening can result either from the temperature of the ions being higher than that of electrons (non-thermal equilibrium) or from small-scale velocity fields induced by wave and turbulent motions. 


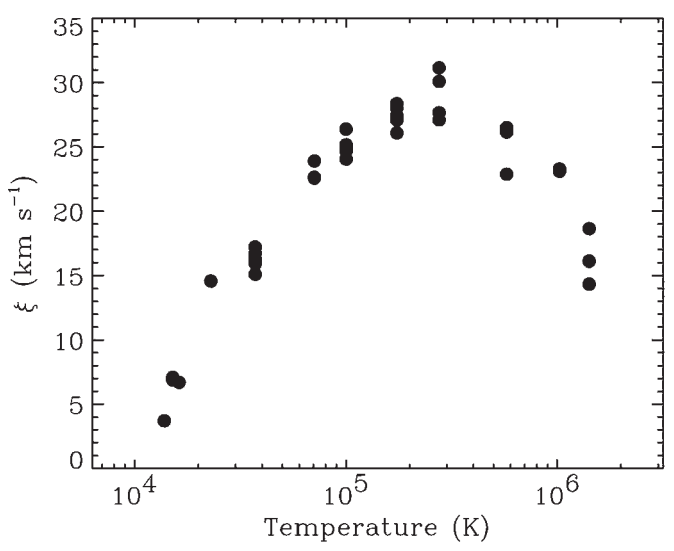

Figure 2.6: Observations of non-thermal velocity versus temperature in the quiet Sun. After Chae et al. (1998a)

In the quiet Sun, the non-thermal velocity was found to be about $5-10 \mathrm{~km} \mathrm{~s}^{-1}$ in the upper chromosphere and to increase further with the temperature (see,e.g., Mariska et al., 1978). At a temperature of about $3 \times 10^{5} \mathrm{~K}$, Warren et al. (1997b) measured an average non-thermal velocity of $34 \mathrm{~km} \mathrm{~s}^{-1}$ for O VI in the quiet Sun. In the upper transition region and corona, Mariska (1992) summarized previous observations and concluded that the non-thermal velocity must peak at some temperature in the upper transition region and then either remain roughly constant or begin to decrease in the corona. Moreover, using SUMER data, Chae et al. (1998a) found that line widths begin to decrease above some temperature at about $3 \times 10^{5} \mathrm{~K}$ (see Figure 2.6.

In the coronal hole, Dere et al. (1989b) deduced the line widths of C IV profiles from HRTS and showed that the non-thermal broadening is only marginally greater in the coronal hole than in the quiet Sun. The average most probable values are $24 \mathrm{~km} \mathrm{~s}^{-1}$ in the quiet Sun and $25 \mathrm{~km} \mathrm{~s}^{-1}$ in the coronal hole. Using SUMER data, similar results were also obtained by several authors (Lemaire et al., 1999; Stucki et al., 2000b; Wilhelm et al., 2002). Wilhelm et al. (2002) found that the Mg IX line has a Doppler width of $151 \mathrm{~m} \AA$ $\left(v_{1 / e}=64 \mathrm{~km} \mathrm{~s}^{-1}\right)$ in ECH compared to $143 \mathrm{~m} \AA\left(v_{1 / e}=61 \mathrm{~km} \mathrm{~s}^{-1}\right)$ in QS regions.

Above the limb in the polar coronal hole, SUMER observations (see, e.g., Marsch et al., 1997; Wilhelm et al., 1998b; Doschek et al., 2001) showed that some lines have widths increasing with height, and confirmed the previous study by Hassler et al. (1990). However, some lines, for example, the Mg IX and Ne VII have no such tendency. In addition, the widths of the Si VIII, Ne VIII and Mg VIII line grow up to a certain height and then become roughly constant.

\subsubsection{The density and temperature}

The major results for densities and temperatures, which were inferred from a number of observations in or above coronal holes of the pre-SOHO era, have been summarized by 
Habbal et al. (1993); Esser and Habbal (1997). From these studies, the derived electron density is about $3 \times 10^{8} \mathrm{~cm}^{-3}$ on the disk and about $2 \times 10^{8} \mathrm{~cm}^{-3}$ (with a wide range from $0.4-7 \times 10^{8} \mathrm{~cm}^{-3}$ ) just above the limb. The temperature in the coronal hole was concluded to be likely below than $10^{6} \mathrm{~K}$, lower than in surrounding quiet regions.

During the last solar minimum (August - September 1996), an equatorial coronal hole named "Elephant's Trunk", which extended from the north pole down to below the equator, was intensively studied by many authors. Del Zanna and Bromage (1999) analyzed spatially averaged spectra within the hole observed by CDS on SOHO and made use of line intensity ratios to obtain the density, temperature and element abundances inside and outside the coronal hole. It was shown that the coronal hole has coronal electron densities of about $2-3 \times 10^{8} \mathrm{~cm}^{-3}$, a factor of 2 lower than the quiet Sun areas near the hole. The underlying transition region has densities of about $1 \times 10^{10} \mathrm{~cm}^{-3}$ in the network and higher values in the cell center, as being deduced from the $\mathrm{O}$ IV ratio. The temperature of peak emission is found to be at about $8 \times 10^{5} \mathrm{~K}$, compared to about $9.7 \times 10^{5} \mathrm{~K}$ in the quiet Sun.

In addition, the same Elephant's Trunk coronal hole was also observed later in October by the CDS instrument and the Nancay Radioheliograph (France) (Drago et al., 1999). Using the Differential Emission Measure (DEM) derived from EUV line intensities in the radio transfer equation and comparing the results with the observed radio brightness temperatures, Drago et al. (1999) estimated the coronal temperature and electron density at the hole base of about $T_{c} \leq 9 \times 10^{5} \mathrm{~K}$ and $N_{e} \simeq 3 \times 10^{8} \mathrm{~cm}^{-3}$. During the same period, Young and Esser (1999) deduced significantly lower electron densities in the north polar hole. Using the line ratio Mg VIII $315 \AA / 317 \AA$ A obtained by CDS, their studies yield upper limits of $2.3 \times 10^{-7} \mathrm{~cm}^{-3}$ and $6.5 \times 10^{-7} \mathrm{~cm}^{-3}$ from two coronal hole spectra, respectively. It should mentioned that in the work of Young and Esser (1999) only dark regions in the hole were selected. This may be the reason why they got much lower densities.

Just above the limb of a polar coronal hole, Warren and Hassler (1999) measured electron densities using 5 line pairs (Si III, Ne VII, Mg VIII, Si VIII and Mg IX obtained with SUMER) with a formation temperature between $0.03 \mathrm{MK}$ and $1 \mathrm{MK}$. The electron densities were estimated to range from $5.9 \times 10^{8} \mathrm{~cm}^{-3}$ to $1.7 \times 10^{8} \mathrm{~cm}^{-3}$. More detailed measurements above the limb will be discussed in Section 2.4.1,

\subsection{Coronal holes and the fast solar wind}

\subsubsection{The fast solar wind}

\section{Properties of the fast solar wind at $1 \mathrm{AU}$}

According to a summary by Marsch (1999), the solar wind can be generally grouped into three types: the steady fast wind originating on open field regions in coronal holes, the 
unsteady slow wind coming probably from the temporarily open streamer belt and the transient wind caused by large coronal mass ejections.

The average properties at $1 \mathrm{AU}$ of two basic modes of the solar wind, namely, the fast and slow wind, are summarized in Table 2.1 (Axford and McKenzie, 1997). Their detailed morphological and kinetic properties have been reviewed after the Helios mission by Marsch (1991) and Schwenn (1990). According to the in situ observations, some quantities are on average nearly constant for both modes of the solar wind, for example the proton flux and magnetic flux. For instance, Marsch and Richter (1984) found that the proton and magnetic flux ranged from $2-4 \times 10^{8} \mathrm{~cm}^{-2} \mathrm{~s}^{-1}$ and $3.28 \pm 1.49 \mathrm{nT} \mathrm{AU}$ respectively deduced from Helios data. The values are also confirmed by Ulysses observations over the poles. However, the slow wind generally shows highly variable properties, while the fast wind is quite stable and has very constant properties.

We emphasize some observational properties that are of great importance for determining the acceleration mechanism of the fast wind. First, the protons are found to be generally hotter than the electrons, and are anisotropic, with the perpendicular temperature larger than the parallel one. Second, heavy ions, which have nearly mass-proportional temperatures, are hotter than the protons, and are also faster than the protons by about the local Alfvén speed. Third, the electron distribution function shows halo/core structure. In

Table 2.1: Two modes of solar wind flow (after Axford and McKenzie (1997))

\begin{tabular}{l|l|l}
\hline Property (1 AU) & Low Speed & High Speed \\
\hline Speed $(V)$ & $<400 \mathrm{~km} \mathrm{~s}^{-1}$ & $700-900 \mathrm{~km} \mathrm{~s}^{-1}$ \\
Density $(n)$ & $\sim 10 \mathrm{~cm}^{-3}$ & $\sim 3 \mathrm{~cm}^{-3}$ \\
Flux $(n V)$ & $\sim 3 \times 10^{8} \mathrm{~cm}^{-2} \mathrm{~s}^{-1}$ & $\sim 2 \times 10^{8} \mathrm{~cm}^{-2} \mathrm{~s}^{-1}$ \\
Magnetic field $\left(B_{r}\right)$ & $\sim 2.8 \mathrm{nT}$ & $\sim 2.8 \mathrm{nT}$ \\
Temperatures & $T_{p} \sim 4 \times 10^{4} \mathrm{~K}$ & $T_{p} \sim 2 \times 10^{5} \mathrm{~K}$ \\
& $T_{e} \sim 1.3 \times 10^{5} \mathrm{~K}$ & $T_{e} \sim 10^{5} \mathrm{~K}$ \\
Coulomb collisions & important & negligible \\
Anisotropy & $T_{p}$ isotropic & $T_{p}(\perp)>T_{p}(\|)$ \\
Beams & none & fast ion beams + \\
& & electron "strahl" \\
Structure & filamentary, highly variable & uniform, slow change \\
Composition & He/H $\sim 1-30 \%$ & He/H $\sim 5 \%$ \\
Waves & both directions & outwards propagating \\
Minor species & $n_{i} / n_{p}$ variable & $n_{i} / n_{p} \sim$ constant \\
& $T_{i} \sim T_{p}$ & $T_{i} \sim A T_{p}$ \\
& $V_{i} \sim V_{p}$ & $V_{i} \sim V_{p}+V_{A}$ \\
Associated with & streamers, transiently open & coronal holes \\
& field & \\
Sunspot minimum & $\pm 15^{\circ}$ from equator & $>30^{\circ}$ \\
Sunspot maximum & dominant at most latitudes & less frequent \\
\hline
\end{tabular}



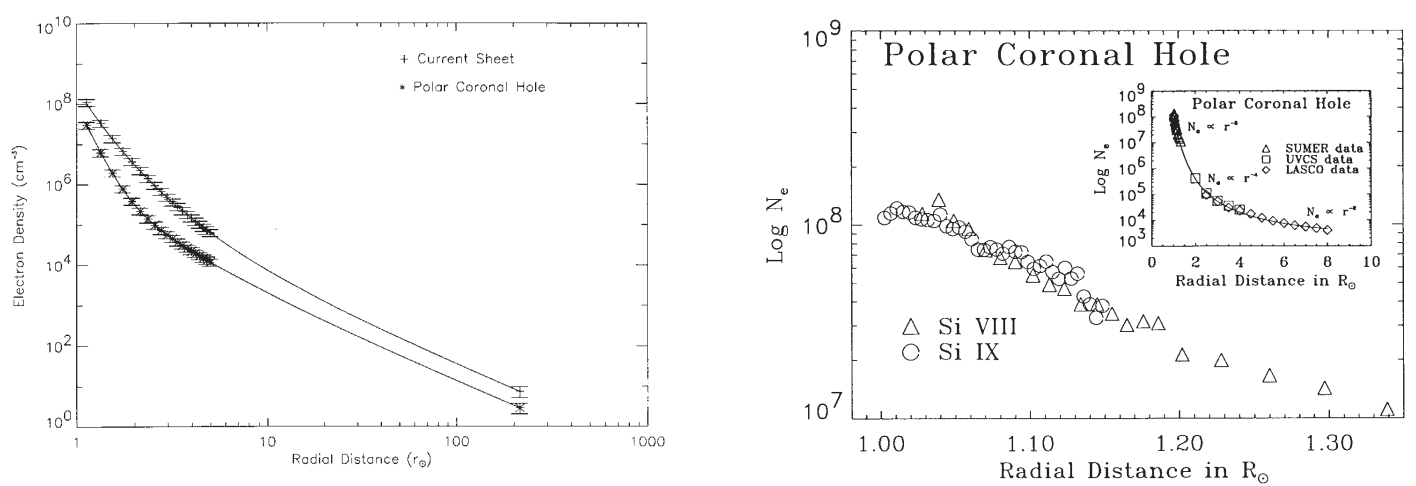

Figure 2.7: Density profile versus heliocentric distance. Left: measurements from Skylab heliograph and SWICS/Ulysses (Sittler and Guhathakurta, 1999); Right: measurements by CDS, SUMER and LASCO on SOHO (Doyle et al., 1999).

addition, Alfvén waves are the major micro-structures in the fast solar wind, with their propagating direction outward. This implies that their source lies on the Sun.

\section{Properties of the fast solar wind near the Sun}

The properties of the fast solar wind near the Sun deduced mainly from observations of CDS, UVCS and SUMER on SOHO have recently been reviewed by Marsch (1999) and Cranmer (2002).

Near the Sun, the electron density varying with the heliocentric distance has been constructed from remote-sensing combined with in-situ observations. Using the Si VIII line ratio (1445 $\mathrm{\AA} / 1440 \AA$ ) obtained by SUMER, Wilhelm et al. (1998b) deduced electron densities in interplume regions to be about $1 \times 10^{8} \mathrm{~cm}^{-3}$ at $1.02 \mathrm{R}_{\odot}$ and drop to about $8 \times 10^{6}$ $\mathrm{cm}^{-3}$ at $1.3 \mathrm{R}_{\odot}$. Their data also show that plume regions are denser than interplume regions. In Figure 2.7, two sets of density profiles are summarized by Doyle et al. (1999) measured with SUMER, CDS and LASCO (left panel) and by Sittler and Guhathakurta (1999) measured with Skylab and Ulysses (right panel), respectively. These two measurements are rather consistent with each other.

A summary of temperature and outflow-velocity measurements was recently given by Cranmer (2002), based on remote-sensing observations (SUMER, UVCS, CDS and Yohkoh), IPS observations and in situ observations (Helios, IMP, Ulysses and Voyager). In Figure 2.8, double sets of curves represent roughly the lower and upper values of each parameter.

From the SUMER and CDS observations, a quite surprising result is that the electrons are rather cold in coronal holes. The electron temperature $\left(T_{e}\right)$ derived by Wilhelm et al. (1998b), who used the line pair Mg IX $749 \AA$ /706 $\AA$ obtained by SUMER, was found to range from $0.75 \mathrm{MK}$ to $0.88 \mathrm{MK}$ at heights between $1.03-1.6 \mathrm{R}_{\odot}$. Using the line pair O VI $173 \AA / 1032 \AA$ obtained from CDS and SUMER, respectively, David et al. (1998) 


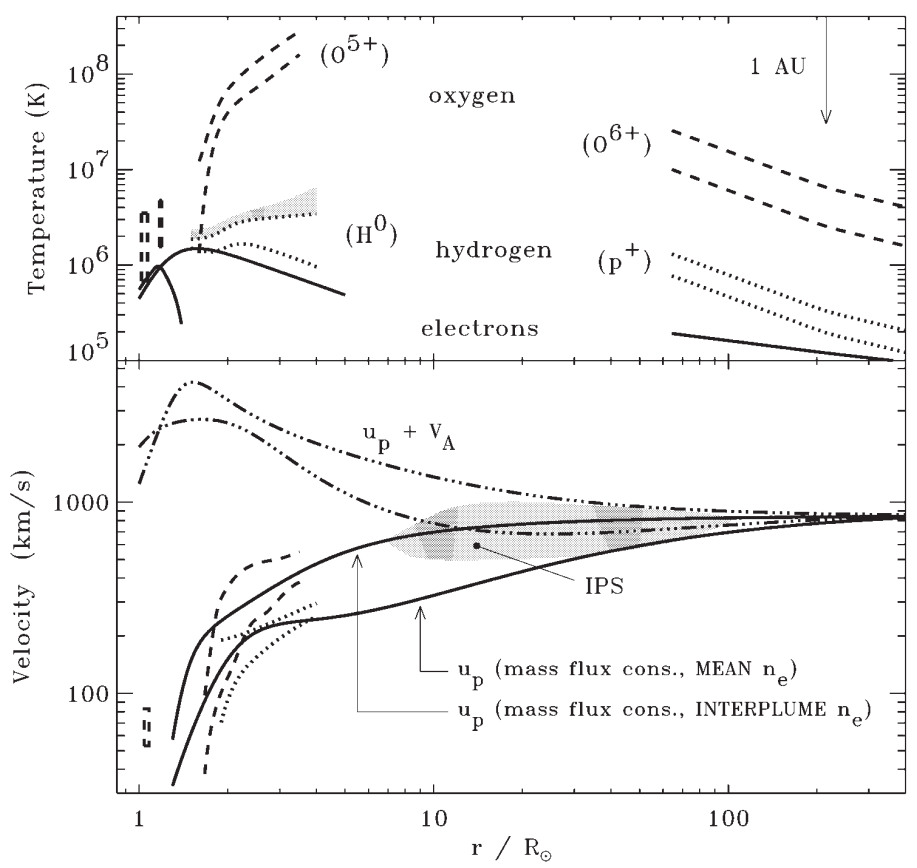

Figure 2.8: Properties of the fast solar wind near the Sun. Upper panel: Temperature profiles of electrons (solid), hydrogen (dotted) and oxygen (dashed); Bottom panel: Velocity profiles of protons (solid), hydrogen (dotted) and oxygen (dashed). After Cranmer (2002).

derived the electron temperature as a function of height $\left(1.0-1.33 \mathrm{R}_{\odot}\right)$ above the limb in a north polar hole (see the lower solid curve in the upper panel of Figure 2.8), as well as in a west equatorial streamer. Their data suggest that in the coronal hole $T_{e}$ has a maximum of less than $1 \mathrm{MK}$ at a height around $1.15 \mathrm{R}_{\odot}$, then falls off again and has a value of about $0.5 \mathrm{MK}$ at $1.3 \mathrm{R}_{\odot}$. However, this trend is somewhat contrary to results deduced from X-ray observation with Yohkoh (Aschwanden and Acton, 2001) and indicated by in situ measurements of ion fractions in the fast solar wind with SWICS/Ulysses (Geiss et al., 1995, Ko et al., 1997), which suggest that the electron temperature can reach a maximum of more than 1.3 MK at 1.5 $\mathrm{R}_{\odot}$ (see discussion in Esser and Edgar, 2000; Cranmer, 2002; Marsch et al., 2003).

SUMER measurements also showed evidence that the ions are much hotter than the electron even very near the Sun (below $1.1 \mathrm{R}_{\odot}$ ). Their temperatures exhibit a slight trend to increase with increasing mass-per-charge number (Tu et al., 1998, 1999).

At heliocentric distance between $1.5-4 \mathrm{R}_{\odot}$, UVCS observations of spectral lines such as $\mathrm{H}$ I Ly $\alpha$ and $\mathrm{O}$ VI can be used to deduce an equivalent thermal velocity by analyzing Doppler widths, and the outflow velocity by the Doppler dimming technique. Above polar coronal holes, these studies indicate that ions are hotter than electrons, and that their temperatures are anisotropic. In particular, the minor ion $\mathrm{O}^{5+}$ has a very high perpendicular kinetic temperature in excess of $100 \mathrm{MK}$ at $3 \mathrm{R}_{\odot}$ and a strong temperature anisotropy with $T_{\perp} / T_{\|} \approx 10-100$ (Kohl et al., 1997, 1998). Moreover, the $\mathrm{O}^{5+}$ and $\mathrm{Mg}^{9+}$ ions are 
also found to be heated more than in mass proportion (Kohl et al., 1999). On the other hand, the outflow velocity as deduced from $\mathrm{O}^{5+}$ is found to be larger than that from $\mathrm{H}^{0}$ (Kohl et al., 1998; Li et al., 1998, Cranmer et al., 1999). The higher outflow velocity and temperature of $\mathrm{O}^{5+}$ as compared to the protons indicate strong preferential heating and acceleration of this minor ion.

In equatorial coronal holes, the UVCS observations exhibited properties different from those in polar coronal holes, however. Outflow velocity and perpendicular thermal velocity observed in a large equatorial coronal hole in 1999 were found to be only $3-4$ and 2 times lower at similar heights in comparison with those observed in polar holes during 1996 and 1997 (Miralles et al., 2001b a). However, in situ observations with the ACE spacecraft indicate that the fast solar wind associated with this hole has a velocity of $600-700 \mathrm{~km} \mathrm{~s}^{-1}$, only about $15 \%$ less than that observed by Ulysses above the poles during the solar minimum.

\subsubsection{Coronal holes: sources of the fast solar wind}

The solar wind has been theoretically modelled and observed since more than four decades. However, its source regions were not known until the beginning of the 1970's. With the development of the space technology, detailed studies of the coronal holes became possible with X-rays and UV spectra. The direct connection of a recurrent, highspeed solar wind stream with a coronal hole was found by Krieger et al. (1973). This was in agreement with theoretical arguments by Pneuman and Kopp (1971) and Noci (1973), who studied the plasma-magnetic field interactions and the energy balance in the coronal hole, respectively. This association between coronal holes and high-speed solar wind streams was a little later confirmed further by a number of authors using increasingly extensive sets of observations (see, e.g., Neupert and Pizzo, 1974; Nolte et al., 1976; Sheeley et al., 1976). During the Skylab workshop, Hundhausen (1977) came to some basic conclusions attained by previous studies. First, large near-equatorial coronal holes can produce high-speed streams observed in the Earth's orbit, with the interplanetary magnetic polarity agreeing with the dominant polarity of the photospheric magnetic field underlying the hole. The observed velocities at $1 \mathrm{AU}$ appear to be closely related with the size of the coronal holes (Nolte et al., 1976). Second, some high-speed streams can be tracked back to regions on the Sun, without pronounced coronal holes near the equator observed in X-rays. Third, small coronal holes may not cause high-speed streams.

For polar coronal holes, direct in situ observation has been carried out by Ulysses, which is the first spacecraft flying over the Sun's poles (see, e.g., Geiss et al., 1995; Woch et al., 1997; McComas et al., 2000). The solar wind speed varying with heliographic latitude is shown in Figure 2.9. Fast solar wind with speeds of about $750 \mathrm{~km} \mathrm{~s}^{-1}$ emanating from the polar coronal holes were found to be dominant during the sunspot minimum. During the solar maximum around the year 2000, the solar wind speed above the south pole was reduced to be about $400 \mathrm{~km} \mathrm{~s}^{-1}$, indicating the absence of the polar coronal hole. All these results directly demonstrate that the fast solar wind comes from the coronal hole. 


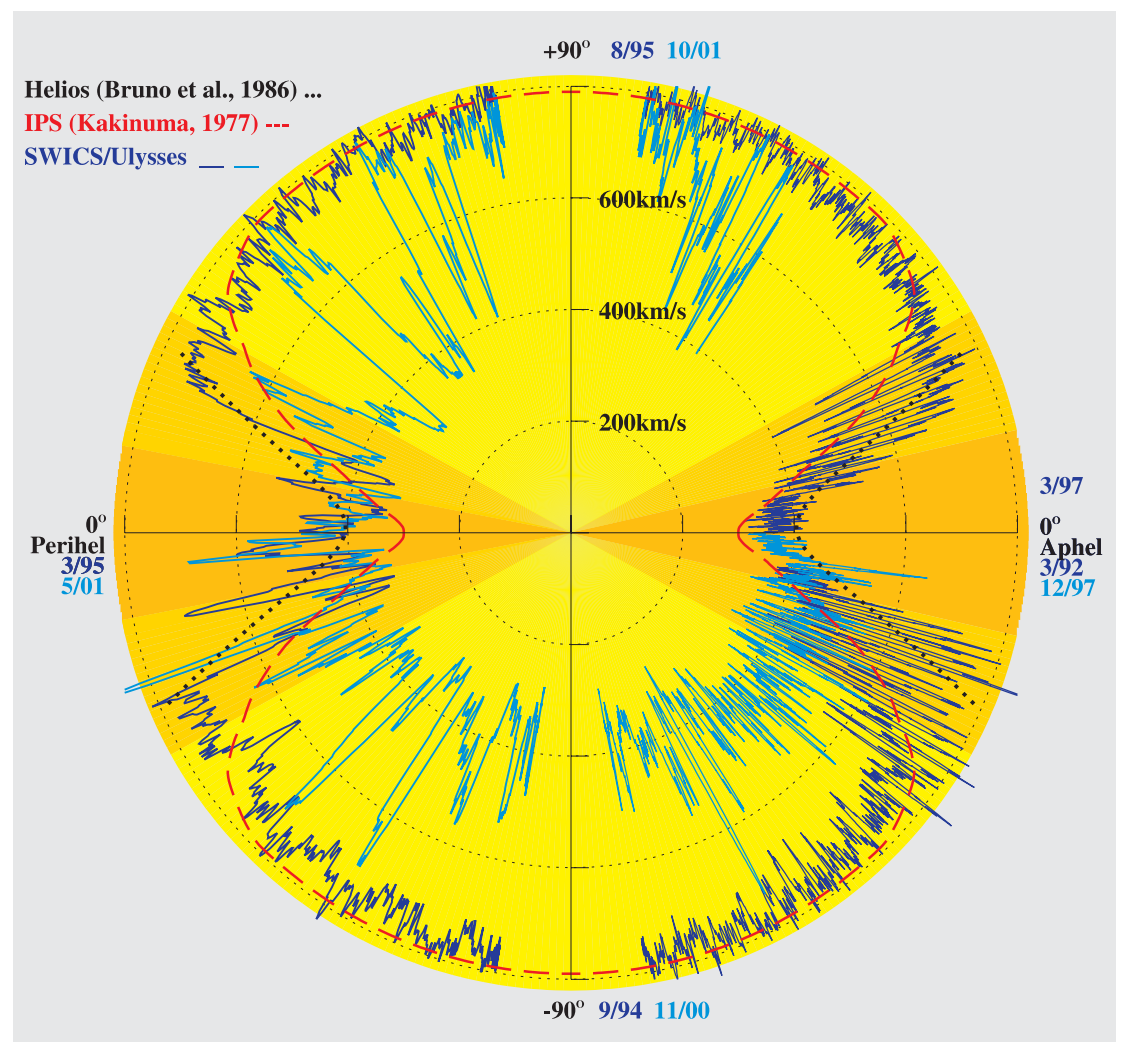

Figure 2.9: SWICS/Ulysses observations: This polar diagram shows that the 1-day average of the proton speed varies with heliographic latitude. The plot comprises SWICS data from March 1992 to October 2001. The speed profiles are also compared with fits to Helios and IPS observations. The highest latitude reached by Ulysses is $80^{\circ}$. After Woch et al. (1997), note that the plot with new data obtained from December 1997 to October 2001 was added later by the authors.

At the base of the coronal hole, a detailed picture concerning the origin of the fast solar wind was first obtained by SUMER observations in the polar coronal hole. The Dopplergram deduced from the line profile of Ne VIII showed strong evidence that the wind originates in the chromospheric network (Hassler et al., 1999). This is consistent with theoretical ideas that the solar wind is initially accelerated in the coronal funnels by dissipation of high-frequency waves, which we will discuss in the next section.

\subsection{Heating and acceleration mechanisms in coronal holes}

\subsubsection{The mass and energy balance}

No doubt, the ultimate source of the solar wind lies in the lower layers of the solar atmosphere. Above the photosphere, the outer solar atmosphere consists of the chromosphere 


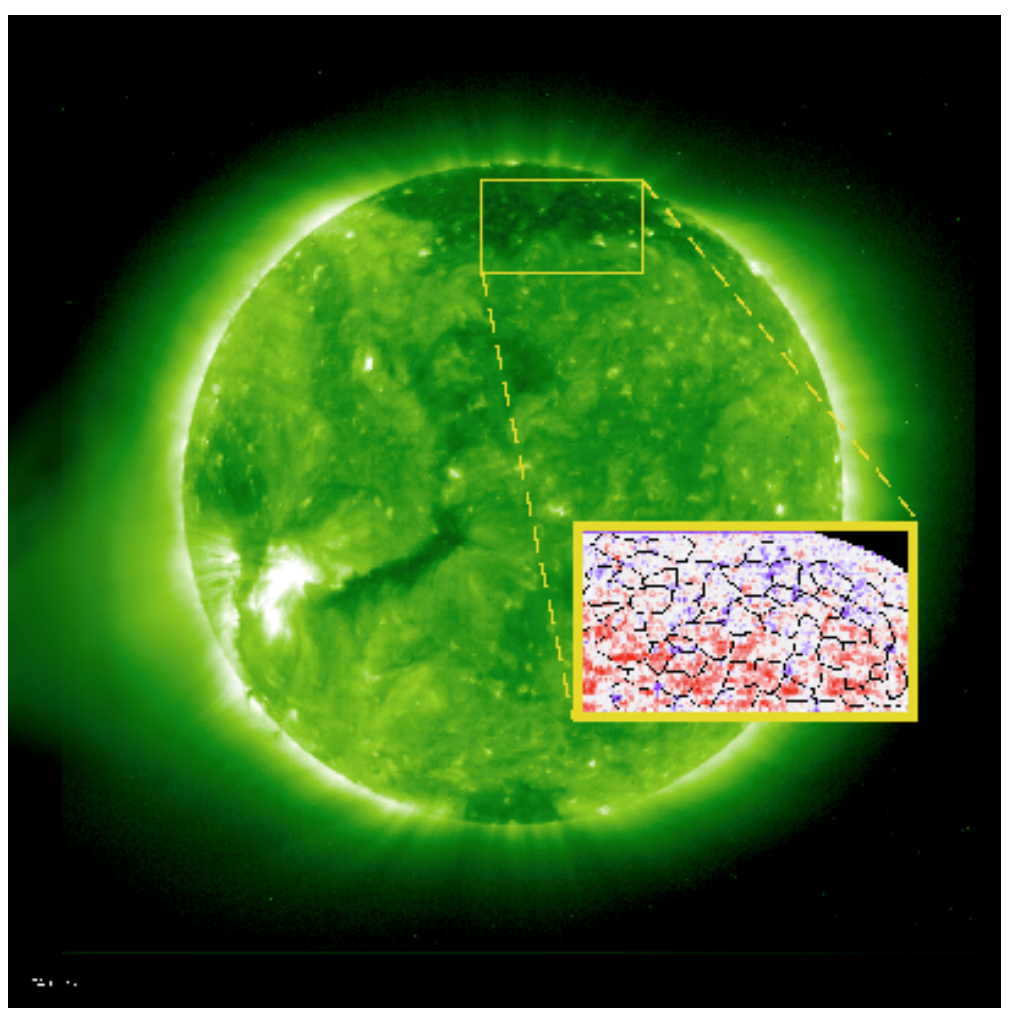

Figure 2.10: Source of the fast solar wind: EIT image at $195 \AA$ shows the corona at solar minimum. A portion of the polar coronal hole insert is shown as a Dopplergram of the Ne VIII line scanned by SUMER. The blue shifts from the chromospheric network are interpreted as outflow of the nascent fast solar wind (Hassler et al., 1999).

and corona, being separated by a very thin transition region. The mass and energy balance in these regions is particularly important to understand the origin of the solar wind. Substantial progress on this issue has been made, based on the measurements by various experiments in FUV/EUV and X-ray wavelengths.

Considering the energy balance from the chromosphere to the inner corona, one concludes that the primary mechanisms responsible for energy loss include radiation, thermal conduction and mass loss in form of the solar wind. Meanwhile, the primary mechanisms responsible for energy deposition are considered in the form of dissipation of waves and magnetic energy, thermal conduction and mass flow. In different layers, these mechanisms may have different effects on the energy balance. Withbroe and Noyes (1977) summarized the various energy losses (given in Table 2.2) for the chromosphere and corona of the quiet Sun, coronal hole and active region, respectively. This summary was based on empirical modelling studies by many authors (see Withbroe and Noyes, 1977, and references therein). Furthermore, the total energy required to heat the corona was suggested to be the same for both coronal holes and the quiet Sun by considering uncertainties of estimates. By using data from remote sensing and in situ observations as constraints, the empirical model indicates that the total non-radiative energy input is about $5 \times 10^{5} \mathrm{erg}$ 
Table 2.2: Chromospheric and coronal energy losses (after Withbroe (1977))

\begin{tabular}{|c|c|c|c|}
\hline Parameter & QS & $\mathrm{CH}$ & AR \\
\hline Transition layer pressure $\left(\mathrm{dyn} \mathrm{cm}^{-2}\right)$ & $2 \times 10^{-1}$ & $7 \times 10^{-2}$ & 2 \\
\hline Coronal temperature $\left(\mathrm{K}\right.$, at $\left.\sim 1.1 \mathrm{R}_{\odot}\right)$ & $1.1 \sim 1.6 \times 10^{6}$ & $10^{6}$ & $2.5 \times 10^{6}$ \\
\hline \multicolumn{4}{|l|}{ Coronal energy losses $\left(\mathrm{erg} \mathrm{cm}^{-2} \mathrm{~s}^{-1}\right)$} \\
\hline Conduction flux $F_{c}$ & $2 \times 10^{5}$ & $6 \times 10^{4}$ & $10^{5} \sim 10^{7}$ \\
\hline Radiative flux $F_{r}$ & $10^{5}$ & $10^{4}$ & $5 \times 10^{6}$ \\
\hline Solar wind flux $F_{w}$ & $\leq 5 \times 10^{4}$ & $7 \times 10^{5}$ & $\left(<10^{5}\right)$ \\
\hline Total corona loss $F_{c}+F_{r}+F_{w}$ & $3 \times 10^{5}$ & $8 \times 10^{5}$ & $10^{7}$ \\
\hline \multicolumn{4}{|l|}{$\begin{array}{l}\text { Chromospheric radiative losses } \\
\left(\mathrm{erg} \mathrm{cm}^{-2} \mathrm{~s}^{-1}\right)\end{array}$} \\
\hline Low chromosphere & $2 \times 10^{6}$ & $2 \times 10^{6}$ & $\geq 10^{7}$ \\
\hline Middle chromosphere & $2 \times 10^{6}$ & $2 \times 10^{6}$ & $10^{7}$ \\
\hline Upper chromosphere & $3 \times 10^{5}$ & $3 \times 10^{5}$ & $2 \times 10^{6}$ \\
\hline Total chromospheric loss & $4 \times 10^{6}$ & $4 \times 10^{6}$ & $2 \times 10^{7}$ \\
\hline Solar wind mass loss $\left(\mathrm{g} \mathrm{cm}^{-2} \mathrm{~s}^{-1}\right)$ & $\leq 2 \times 10^{-11}$ & $2 \times 10^{-10}$ & $\left(<4 \times 10^{-11}\right)$ \\
\hline
\end{tabular}

$\mathrm{cm}^{-2} \mathrm{~s}^{-1}$ and its major portion appears to be dissipated within $1-2 \mathrm{R}_{\odot}$ above the Sun's surface (Withbroe, 1988).

According to these studies, because the measured emission in the coronal hole is very similar to the quiet Sun, the physical properties of the chromosphere in both regions are assumed the same. The chromosphere has relatively low temperatures. Here the radiation losses are predominant and balance the energy input by mechanical heating. In the lower corona below about $2 \mathrm{R}_{\odot}$, electron collisions and high temperatures lead to high thermal conduction. This is particularly true for the quiet Sun, where the strong inward thermal conduction exceeds the combined outward losses by radiation and mass flux, and lead to the establishment of a sharp transition region. In the coronal hole, this inward conduction is reduced significantly, which may reduce the temperature gradient of the transition region. Therefore, here most of the heat source is required only to balance the large energy loss by the solar wind.

\subsubsection{Heating and acceleration mechanisms}

According to in situ observations of the solar wind in interplanetary space, it has been found since a long time that the properties of the fast solar wind cannot be adequately described by models based on classical heat conduction if not extended coronal heating in some form is added (Holzer and Axford, 1970). This extended energy, required to boost the thermal expansion velocity to $500-600 \mathrm{~km} \mathrm{~s}^{-1}$ at distances beyond the sonic point, is estimated to be approximately $1 \times 10^{5} \mathrm{erg} \mathrm{cm}^{-2} \mathrm{~s}^{-1}$ (see, Parker, 1991, and references therein). On the other hand, the energy flux that must be deposited for the compensation of the total loss of the corona is about $5 \times 10^{5} \mathrm{erg} \mathrm{cm}^{-2} \mathrm{~s}^{-1}$, and its major portion appears 

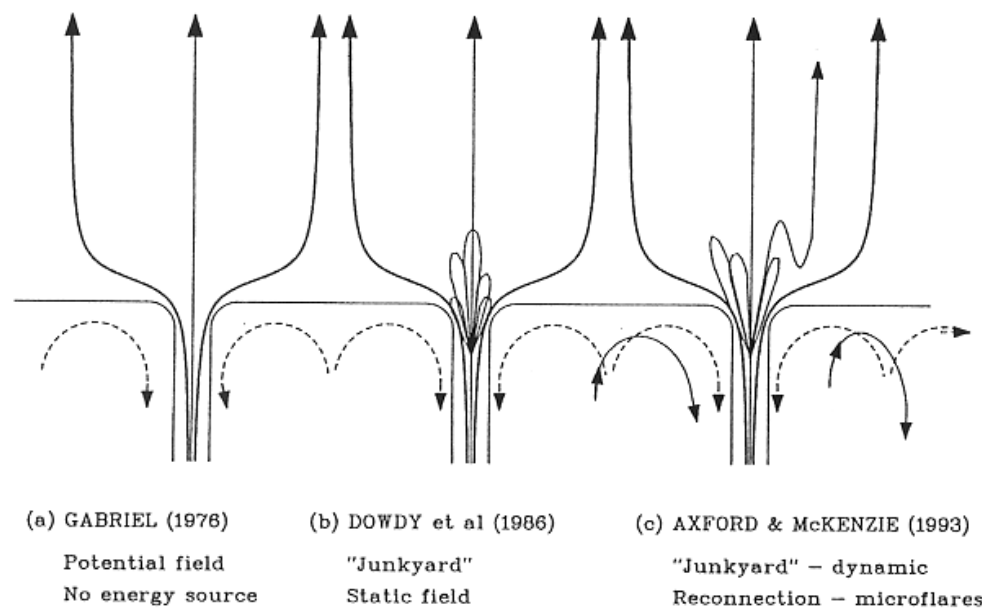
(c) AXFORD \& MCKENZIE (1993)
"Junkyard" - dynamic
Reconnection - microflares
Waves $\rightarrow$ out
Loops $\rightarrow$ down
New flux fed in at sides

Figure 2.11: (a) Gabriel's model; (b) Dowdy's model; (c) Magnetic furnace: magnetic reconnection occurs continuously in the funnels, releasing energy and plasma into the open field lines and also creating loops with hot trapped plasma. The destroyed magnetic flux is continuously replenished by flux advection through the supergranulation flow. After Axford et al. (1999).

to be dissipated within $1-2 \mathbf{R}_{\odot}$ above the Sun's surface, as discussed above (Withbroe, 1988).

The proposed physical processes responsible for heating the corona near the Sun can be basically grouped into two types: current dissipation and wave dissipation. Plasma may be heated by ohmic dissipation of the currents generated by magnetic reconnection. Plasma waves may dissipate via collisions, e.g., viscosity, thermal conduction and electrical resistivity, and/or via kinetic wave-particle interactions. Generally, acoustic waves and slowmode MHD waves are easily damped before they reach the corona, while Alfvén waves and fast-mode MHD waves are considered to be the only modes that can survive in the corona. Unfortunately, low-frequency Alfvén waves are not considered as heating source of the corona near the Sun because of their large dissipation lengths. However, dissipation of high-frequency Alfvén waves is suggested to be a candidate for heating this part of the corona (see, e.g., Marsch, 1992; Axford and McKenzie, 1992, 1997). The key point of this model is that the high-frequency Alfvén waves can be strongly absorbed at the cyclotron frequency of ions in a rapidly declining magnetic field (frequency sweeping), leading to fast heating near the Sun. This cyclotron-resonant heating process was demonstrated to work well since very long time for the distant solar wind (Marsch et al., 1982). Preferential heating and acceleration of the ions very near the Sun, which have been found by observations with UVCS and SUMER, show that this idea may also be applied to the corona.

Although the detailed processes concerned the form of energy dissipations are still un- 
known, there is a general agreement that the ultimate heating source is the activity of the network magnetic fields (network flares) (see, e.g., Parker, 1991; Axford and McKenzie, 1992, 1997; Marsch et al., 2003). It is suggested that these flares release energy (in the form of waves, shocks and energetic particles) via magnetic connection driven continually by photospheric magnetoconvection resulting from the granulation and supergranulation. Such a dynamic picture of the network is described in Figure 2.11, which was developed from the static models suggested by Gabriel (1976) and Dowdy et al. (1986). Different types of such flares have been named as "picoflares", "nanoflares" and "microflares". They are essentially similar concepts but have different characteristic scales, with typical energies ranging from $10^{21} \mathrm{erg}$ to $10^{26} \mathrm{erg}$, and sizes from about $10-100 \mathrm{~km}$ to $2000 \mathrm{~km}$. The "picoflare" with the lowest energy and smallest size, a concept recently suggested by Marsch et al. (2003), is expected to be able to produce waves with high frequencies of $1-10000 \mathrm{~Hz}$, which are required for heating by the cyclotron-resonant damping mechanism.

\subsubsection{Recent modelling studies of the coronal funnels}

Various models have been developed to study the heating and acceleration of the plasma associated with coronal holes and unipolar magnetic fields. Basic equations of the fast solar wind generally comprise the conservation equations for mass, momentum, energy and magnetic flux. The boundary conditions are usually chosen in accordance with remote sensing or in situ observations. However, additional pressure term in the momentum equation and additional heating term in the energy equation, as well as the choice of the boundary conditions, are treated differently by various authors. A detailed discussion about the various models can be found in recent reviews (Marsch, 1999, Marsch et al., 2003).

Models with simultaneous treatments of the chromosphere, transition region and corona associated with the nascent fast solar wind have been developed by various authors (see, e.g., Hammer, 1982, Withbroe, 1988, Hansteen and Leer, 1995, Lie-Svendsen et al., 2002). However, these models contained neither the detailed physical processes of the heating nor the detailed magnetic field configurations in the funnels.

Recently, some modelling work has been done to study the physical properties in a coronal funnel. Coronal heating and acceleration was considered as being due to cyclotron damping of Alfvén waves and thrusting by Alfvén wave pressure (see, e.g., Marsch and Tu, 1997; Hackenberg et al., 2000; Li, 2002; Vocks and Marsch, 2002). The resulting plasma properties depend largely on the geometry of the funnel and the details of the wave-energy transport and dissipation process. According to these studies, the plasma can be driven to a flow speed of several tens of $\mathrm{km} \mathrm{s}^{-1}$, essentially by a large thermal pressure gradient at the base of the funnel. This pressure gradient results from the quick expansion of the magnetic flux tube, causing rapid heating with height through the high-frequency wave sweeping (resonant absorption) mechanism. 



\section{Chapter 3}

\section{Instrumentation and Diagnostic Principles}

\subsection{Introduction}

\subsubsection{Overview of observations at FUV/EUV wavelengths}

Traditionally, the spectrum with wavelengths ranging from $100 \AA$ to $2000 \AA$ is part of the vacuum-ultraviolet (VUV). They can be further divided into extreme-ultraviolet (EUV) with wavelengths from $100 \AA$ to $1200 \AA$ and far-ultraviolet (FUV) with wavelengths from $1200 \AA$ to $2000 \AA$ (Wilhelm, 2003). Measurements of FUV/EUV spectra emitted by the Sun can provide us with a powerful diagnostic tool to study essential physical processes occurring in the upper solar atmosphere. An obvious advantage from the coronal point of view is that the radiation from the photosphere, due to its low temperature, dominates only in visible wavelengths, while the FUV/EUV emission originates mainly in the overlying layers including the chromosphere, transition region and low corona. Therefore, detailed observations of such regions become possible even against the solar disk. However, the traditional ground-based observations can not be made in FUV/EUV wavelengths, owing to their strong absorption by ozone and other molecules in the Earth's atmosphere. Thus, such measurements can only be made with instruments on board sounding rockets or spacecrafts flying at an altitude of at least $150 \mathrm{~km}$ (Noyes, 1982). Consequently, detailed studies of upper solar atmosphere with FUV/EUV spectra were highly restricted before the space era.

The first ultraviolet observations of the Sun from space were made, using a German V2 rocket, on 10 October 1946 by US Naval Research Laboratory (NRL) (Baum et al., 1946). Spectrograms with a wavelength range from $2200 \AA$ to $3400 \AA$ were obtained at various altitudes from 1 to $88 \mathrm{~km}$. Since then, many space programs, with instruments both on sounding rockets and spacecrafts, have been carried out. These programs included early missions, such as OSO, Skylab, SMM, Spacelab and a large number of rocket-borne experiments, and recent missions as SOHO and TRACE. With the development of the experimental technology, great progress has been made in this research field. Highlights of the previous achievements using various instruments at FUV/EUV wavelengths have been reviewed by some authors (see, e.g., Mariska, 1992; Mason and Monsignori Fossi, 1994; Wilhelm, 2003).

Many space-based and rocket-borne missions had been launched before the Skylab time, which include the Orbiting Solar Observatory (OSO) series. The instruments onboard generally had both low spectral resolutions (larger than $0.1 \AA$ ) and low spatial resolutions (larger than $5^{\prime \prime}$ ). For example, many spectral lines with wavelengths between $300 \AA$ and $1400 \AA$ were observed by the Harvard College Observatory (HCO) spectroheliometer 
S055 on OSO-4 and OSO-6 (cf. Huber et al., 1973), with a spectral resolution of $1.6 \AA$. These measurements were used to model the chromosphere, transition region and corona.

Since the Skylab time, many instruments, such as the S082A slitless spectrograph and S082B slit spectrograph on Skylab/ATM, UVS and MCS on OSO 8, UVSP on SMM, HRTS on board both Spacelab 2 and rockets, and so on, were operated with high spectral and/or spatial resolutions. With instruments on Skylab/ATM, coronal holes were intensively studied at EUV wavelengths, combined with observations of X-ray, white light and photospheric magnetic fields (Zirker, 1977). Moreover, systematic outflows in coronal holes were deduced from line profiles in the wavelength range 610-630 $\AA$, which were observed by an EUV sounding rocket spectrometer (Rottman et al., 1982; Hassler et al. 1990, 1991). Another instrument - the NRL/High Resolution Telescope and Spectrograph (HRTS) should be mentioned here, because it had both high spectral resolution $(0.05 \AA)$ and high spatial resolution $\left(1^{\prime \prime}\right)$. HRTS was operated at wavelengths longer than $1150 \AA$. Many important phenomena occurring in the transition region, such as systematic redshifts, explosive events and fine structures, were first studied with HRTS (see, e.g., Bartoe and Brueckner, 1975; Brueckner et al., 1986; Dere et al., 1987, 1991).

Although previous studies have provided us with a wealth of information about the outer solar atmosphere, there is no doubt that further observations with high spatial resolution, high time resolution and wide spectral coverage are needed because of the highly structured and dynamic nature of the solar atmosphere. The successful launch and operation of SOHO provides such a possibility. In particular, the SUMER instrument has a capacity combining high time, spectral and spatial resolutions, and moreover, wide spectral coverage.

\subsubsection{SOHO mission}

The Solar and Heliospheric Observatory (SOHO) is one of the space missions of the Solar Terrestial Science Programme (STSP) and was built and is operated together by the European Space Agency (ESA) and the National Aeronautics and Space Adminstration (NASA). SOHO was launched on 2 December 1995 and was then put into a halo orbit with a period of 180 days around the first Lagrangian point (L1) on 14 February 1996. This L1 point is located in an orbit between the Sun and Earth and about 1.5 million kilometers away from the Earth, where the centrifugal forces and gravitational forces of Sun and Earth balance. Unlike observatories in Earth's orbit, SOHO can continuously observe the Sun for the whole day without the limitations caused by day and night effects.

The general propose of the spacecraft SOHO is to study the Sun, from its inner core to the outer corona, and the solar wind by using both remote sensing and in situ observations. The three main scientific goals of the SOHO mission can be summarized as follows (Domingo et al., 1995):

(1) Study of the solar interior, using the techniques of helioseismology

(2) Study of the heating mechanisms of the solar corona, and 


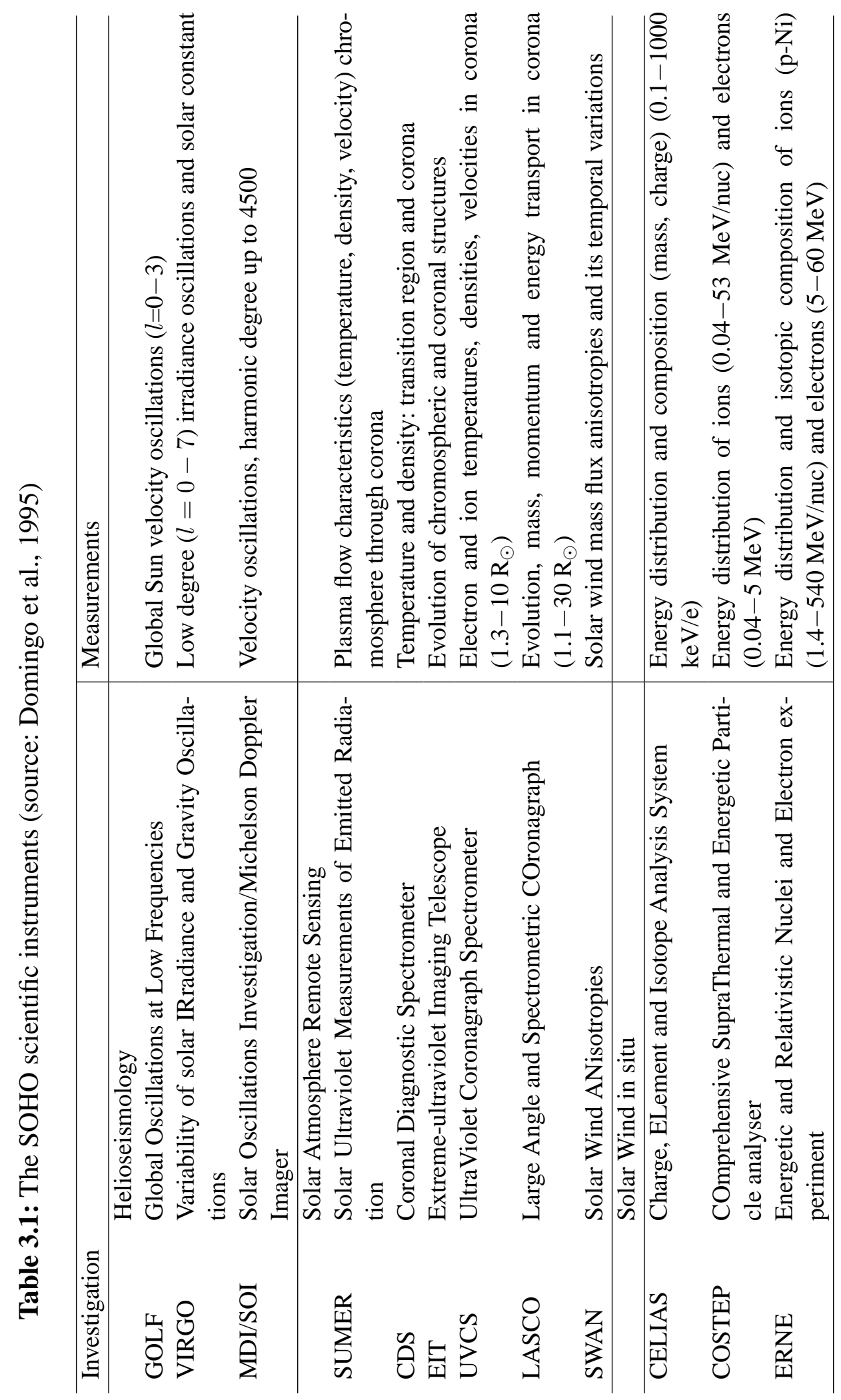


(3) Investigation of the solar wind and its acceleration processes.

Corresponding to these three scientific goals, 12 instruments on board SOHO can be divided into three main groups: helioseismology instruments, solar atmosphere remotesensing instruments, and solar wind in situ instruments. A summary of these instruments is given in Table 3.1 .

The three helioseismology instruments GOLF, VIRGO and MDI/SOI are responsible for measurements of solar oscillations with a high precision and accuracy. Among them, the MDI/SOI can provide high precision solar images $(1024 \times 1024)$ of the longitudinal magnetic field components, which can be used together with SUMER observations to study various phenomena on the solar disk.

The solar atmosphere remote-sensing instruments include CDS, EIT, SUMER, UVCS, LASCO and SWAN. All these instruments can be run together to study the physical processes that take place in the upper solar atmosphere.

The three solar wind in situ instruments are CELIAS, COSTEP and ERNE, which are designed to measure the key parameters of the solar wind as well as high-energy particles originating in the solar atmosphere.

\subsection{The SUMER instrument}

\subsubsection{Scientific goals of SUMER}

Solar Ultraviolet Measurements of Emitted Radiation (SUMER) is one of 12 instruments on SOHO. As mentioned in Section 3.1.2, two of the important goals of the SOHO mission are to understand the process of heating the corona and the mechanism of accelerating the solar wind. Previous FUV/EUV observations discussed in Section 3.1.1 usually had a spatial resolution of larger than $5^{\prime \prime}$. Such a resolution may be adequate to study the average properties of relatively large-scale features like active regions and coronal holes, but it is clearly inadequate for studies of the detailed structure and dynamics of the upper solar atmosphere. On the other hand, with regard to the spectral coverage, the wavelength region below $1100 \AA$ was only poorly observed before the SOHO mission (Wilhelm et al. 1997).

SUMER with its capabilities was designed to provide us with measurements at both high spatial resolution, high spectral resolution, and also a wide spectral coverage ranging from $500 \AA$ to $1600 \AA$. SUMER measures profiles and intensities of FUV/EUV lines emitted by the solar atmosphere from the upper chromosphere to the lower corona; determines line broadenings, spectral positions and Doppler shifts with high precision and accuracy; provides stigmatic images of selected areas of the Sun in the FUV/EUV with high spatial, temporal and spectral resolution and obtains full images of the Sun and the inner corona in selectable spectral lines, corresponding to a temperature from $10^{4} \mathrm{~K}$ to above $2 \times 10^{6} \mathrm{~K}$. Figure 3.1 shows important emission lines in a temperature versus wavelength plot. 


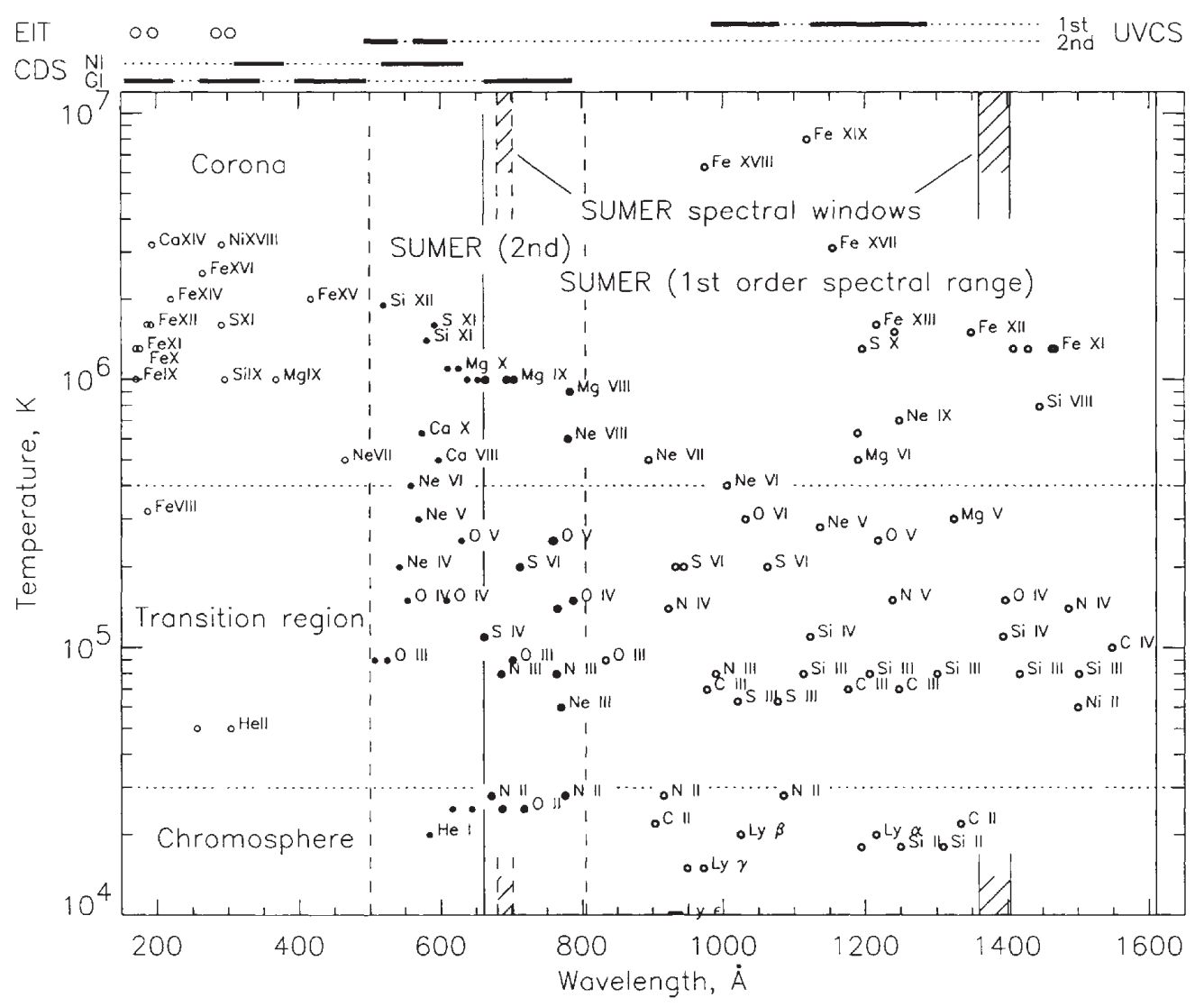

Figure 3.1: Important selected emission lines in the wavelength range from $150 \AA$ to $1610 \AA$ together with the corresponding wavelength coverage of SUMER and other instruments. From Wilhelm et al. (1995)

The SUMER observations permit detailed spectroscopic diagnostics of plasma densities, temperatures and abundances in many solar features, and support detailed studies of underlying physical processes occurring in the upper chromosphere, the transition region and the low corona, including plasma flows, turbulence and wave motions, structures and events associated with solar magnetic activity, atmospheric heating, and also the origin and acceleration of the solar wind in the inner corona.

\subsubsection{The SUMER spectrometer}

SUMER is a stigmatic normal-incidence spectrometer. Its main optical components consist of two parabolic mirrors, a plane mirror and a spherical concave grating, all made out of silicon carbide $(\mathrm{SiC})$ and mounted in an aluminium housing which serves as the optical bench. Figure 3.2 displays the optical layout of this instrument. The Sun can be imaged 


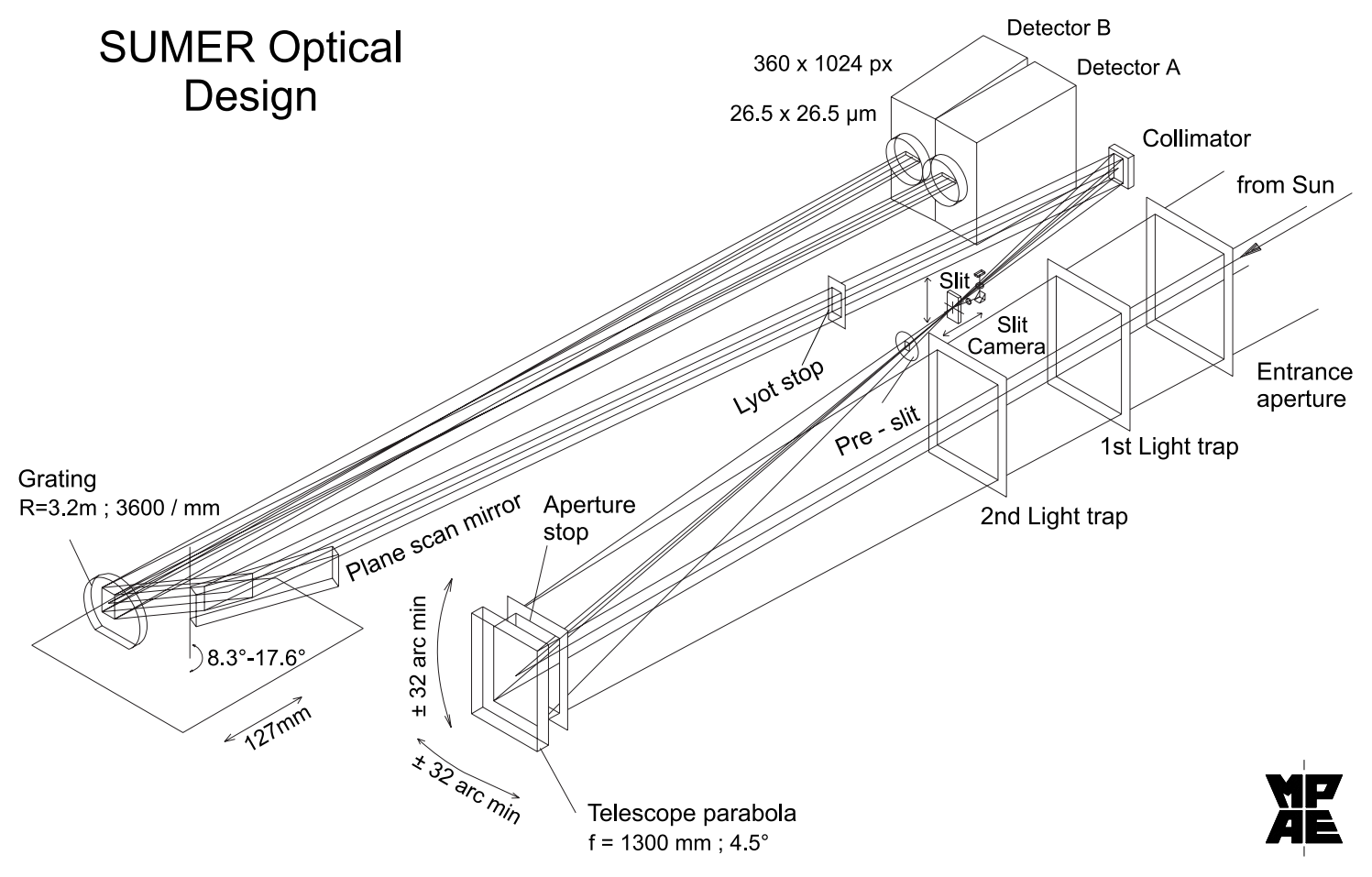

Figure 3.2: The optical layout of the SUMER instrument showing the extreme rays as they propagate through the system (Wilhelm et al., 1995).

into spectrometer entrance slit plane by the first off-axis telescope parabola. This telescope parabola has pointing and scan capabilities (the fastest rate is 300 steps per second with $0.375^{\prime \prime}$ per step). After the beam leaves the slit, it is collimated by the second offaxis parabola, and deflected by the plane mirror onto the grating. This plane mirror can be rotated and thereby changes the incidence angle from $16.74^{\circ}$ to $34.97^{\circ}$, corresponding to a rotation of the angle from $8.4^{\circ}$ to $17.5^{\circ}$ with respect to the incident beam. Thus the required spectral wavelength range of the instrument can be fully covered. In the focal plan of the grating, two two-dimensional detectors are placed to collect monochromatic stigmatic images of the entrance slit. The complete optical design of the instrument is also equipped with a baffle system consisting of an entrance aperture, light traps, an aperture stop, a pre-slit and a Lyot stop. In addition, a Rear Slit Camera (RSC), which serves as pointing verification, is designed to work at visible light wavelengths.

The SUMER spectrometer has imaging capability to build up spectroheliograms or maps of the Sun. One spatial dimension of the image is provided at one exposure by a long slit, whose image can be focused on the detector because the spectrometer is stigmatic. The other spatial dimension is produced by scanning perpendicular to the long extension of the slit. The SUMER spectrometer is equipped by a slit assembly at the focus of the telescope. There are four different slits, which are numbered in the SUMER software and documents. They are all narrow in the direction of the wavelength dispersion to ensure good wavelength resolution. Slit 1 with dimensions $4 \times 300 \operatorname{arcsec}^{2}$ can be selected for observations off the limb to improve counting statistics at the expense of spatial and spec- 


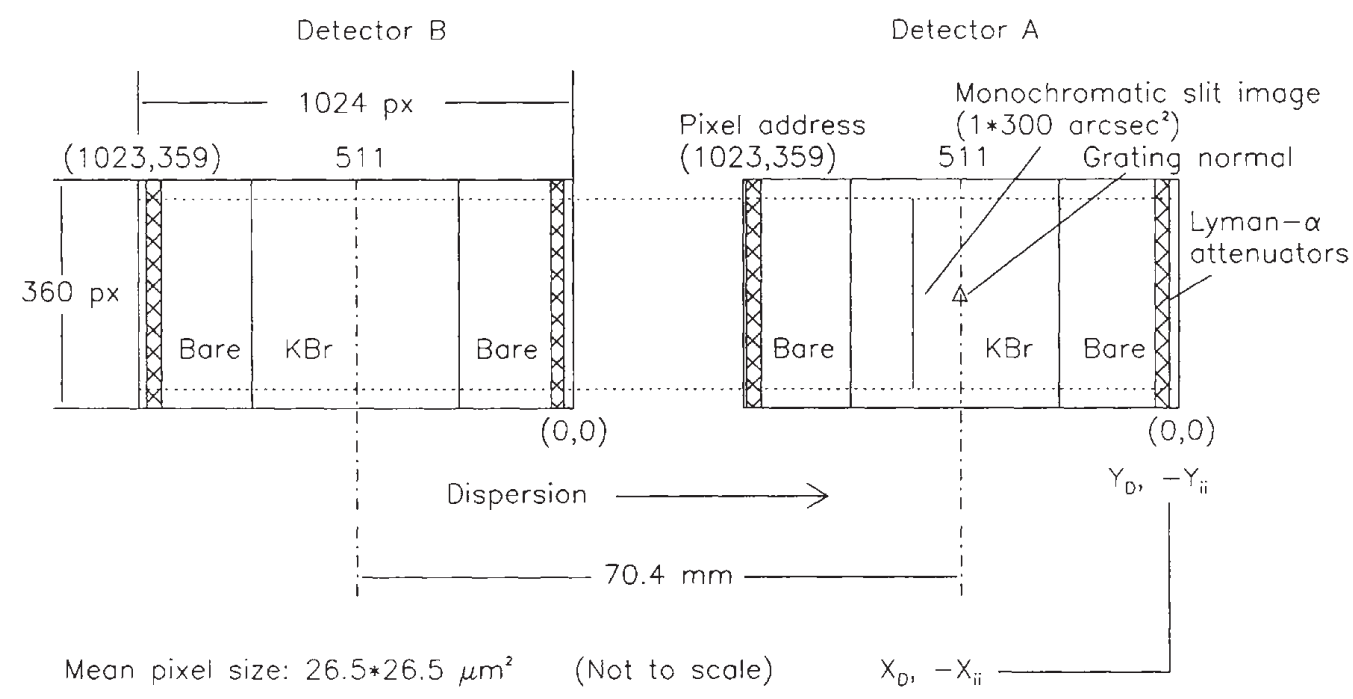

Figure 3.3: Detector arrays in the focal plane of the grating. They have very similar characteristics as far as pixel and photocathode arrangements are concerned, but only detector $A$ is centred on the grating normal with optimum optical performance in the Wardsworth mount. Detector $B$ is offset by $70.4 \mathrm{~mm}$. Only one detector can be switched on at a time. The Lyman- $\alpha$ attenuators consist of grids in front of the bare microchannel plates. Note that the slit image is shorter than the spatial extension of the detector. The "dark" pixels will be useful for scattered light measurements. From Wilhelm et al. (1995)

tral resolutions. However, this slit must not be used for on-the-disk observations. Slit 2 with $1 \times 300 \operatorname{arcsec}^{2}$ is the standard slit to obtain the best possible spectral and spatial resolution image. Slit 4 with $1 \times 120 \operatorname{arcsec}^{2}$ can be used for intense lines. Finally, Slit 7 with $0.3 \times 120 \operatorname{arcsec}^{2}$ is designed for very intense lines or high continuum regimes. The short slits with length of 120 arcsec can be positioned off the center of the detector, i.e., Slit 3 and 5 with a same size as Slit 4, and Slit 6 and 8 with a same size as Slit 7 can be positioned on the top and bottom part of the detector, respectively.

The detector arrangement in the focal plane of the SUMER grating is shown in Figure 3.3. Two detectors are placed there along the dispersion direction with the grating normal pointing to the center of the detector A. The detector B is offset by $70.4 \mathrm{~mm}$. Only one detector can be operated at a time. They are two-dimensional photo-counting devices with a low-resistance Z stack (three MCP wafers stacked on each other) of microchannel plates (MCP) providing detection and amplification, and with a multilayer, cross-delayline (XDL) anode accomplishing readout. Each of them has the size of $27.0 \mathrm{~mm}$ along the dispersion direction and $9.5 \mathrm{~mm}$ along the spatial direction, and is electronically digitized to 1024 (spectral) $\times 360$ (spatial) pixels each of approximately $26.5 \times 26.5 \mu \mathrm{m}^{2}$ in size. In order to increase the detection quantum efficiency (DQE), the central areas of both detectors are coated with $\mathrm{KBr}$ (potassium bromide), which extend from pixel 280 to 770 
for detector A and from pixel 270 to 758 for detector B. Bare MCP areas are arranged on either side of $\mathrm{KBr}$ photocathode from pixel 23 to 56 and from 982 to 1016 for detector A and from pixel 23 to 53 and from 970 to 1005 for detector B. In addition, Lyman $\alpha$ attenuators are arranged at both extreme ends of two detectors and provide a 1:10 attenuation for H I Lyman- $\alpha$ observations. Such a design can satisfy the requirements for various scientific studies, e.g., the $\mathrm{KBr}$ portion will be used for most studies due to its highest efficiency, the bare MCP portion will be used to avoid saturation for very intense lines, and ratio of the intensity as measured with $\mathrm{KBr}$ and with the bare MCP parts can be used to determine whether an unidentified spectral line is being seen in 1st or 2nd order. Nevertheless, the detecting efficiency varies greatly with different portions, i.e., $\mathrm{KBr}$, bare MCP and Lyman- $\alpha$, on the detector and also with different wavelengths. For example, the $\mathrm{KBr} \mathrm{QDE}$ is $\sim 30 \%$ at $584 \AA, \sim 28 \%$ at $1066 \AA$, and $\sim 10 \%$ at $1360 \AA$, while the bare MCP QDE is $\sim 8 \%$ at $584 \AA, \sim 5 \%$ at $1024 \AA$, and $<1 \%$ at $1215 \AA$. The total sensitivity of SUMER will be discussed in Section 3.2.3.

Table 3.2 shows the basic optical characteristics of the SUMER telescope and spectrometer. Note that $1^{\prime \prime}$ at L1 corresponds to on average $715 \mathrm{~km}$ on the Sun (from the Earth $1^{\prime \prime}$ corresponds to on average $726 \mathrm{~km}$ on the Sun). In addition, the magnification factor is the ratio of the effective grating focal length and the collimator focal length.

Theoretically, we can deduce the characteristics of the SUMER spectrometer from the grating equation and the geometric configuration. The grating equation can be written as

$$
m \lambda=d(\sin \theta+\sin \alpha)
$$

where $\theta$ is the angle of incidence on the grating, $\alpha$ is the angle of reflection off the grating, $m$ is the order of diffraction with an always positive value for SUMER, $d$ is the grating spacing which equals to $2777.45 \AA$ corresponding to 3600.42 lines per mm of grating ruling. In order to express the pixel position of a particular wavelength $\lambda$ in the detector plane, we need to know the effective focal length of the grating $f_{\lambda}$, which depends on the angle of incidence. It is given by

$$
f_{\lambda}=\frac{r_{a}}{1+\cos \theta}
$$

where $r_{a}$ is the actual radius of the spherical concave grating, for SUMER, $r_{a}=3200.78$ $\mathrm{mm}$. Thus, the relationship between the pixel position on the detector plane and the angle of reflection can be written as

$$
\tan \alpha=-\frac{\left(n_{p x}-511\right) \Delta_{p x}+\Delta_{d e t}}{f_{\lambda}},
$$

where $n_{p x}$ is the pixel address $\left(0 \leq n_{p x} \leq 1023\right), \Delta_{p x}$ is the size of pixel (about 0.0265 $\mathrm{mm}$ ), $\Delta_{\text {det }}$ equals $0 \mathrm{~mm}$ for detector A and $70.4 \mathrm{~mm}$ for detector B. Therefore, given an incidence angle $\theta$, it is convenient to calculate the wavelength coverage on both two detectors in terms of Eq. 3.1 and 3.3. When the incidence angle $\theta$ varies from $16.74^{\circ}$ to 
Table 3.2: The performance of SUMER instrument (source: SUMER team)

\begin{tabular}{|c|c|}
\hline $\begin{array}{l}\text { The Telescope: } \\
\text { Focal length } \\
\text { Plate scale in slit plane } \\
\text { Total dynamic field-of-view } \\
\text { Smallest step size } \\
\text { (N-S and E-W) }\end{array}$ & $\begin{array}{l}1302.77 \mathrm{~mm} \text { at } 75^{\circ} \mathrm{C} \\
6.316 \mu \mathrm{m} / \operatorname{arcsec} \\
64 \times 64 \operatorname{arcmin}^{2} \\
0.38 \operatorname{arcsec}\end{array}$ \\
\hline The Slits: & $1 \times 300,1 \times 120,0.3 \times 120,4 \times 300 \operatorname{arcsec}^{2}$ \\
\hline \multicolumn{2}{|l|}{ The Spectrometer: } \\
\hline Detector A & $\begin{array}{l}390-805 \AA \text { (2nd order }) \\
780-1610 \AA \text { (1st order }) \\
330-750 \AA \text { (2nd order }) \\
660-1500 \AA \text { (1st order })\end{array}$ \\
\hline Collimator focal length & $399.60 \mathrm{~mm}$ \\
\hline Off-axis angle & $7^{\circ}$ \\
\hline Grating radius & $3200.78 \mathrm{~mm}$ \\
\hline Grating ruling & 3600.42 lines $/ \mathrm{mm}$ \\
\hline $\begin{array}{l}\text { Magnification factor } \\
\text { in detector plane }\end{array}$ & 4.092 at $800 \AA, 4.407$ at $1600 \AA$ \\
\hline \multicolumn{2}{|l|}{ The Detectors: } \\
\hline Array size & $1024($ spectral $) \times 360($ spatial $)$ pixels \\
\hline Pixel size (mean value) & $26.5 \times 26.5 \mu \mathrm{m}^{2}$ \\
\hline Angular scale & $1.03 \mathrm{arcsec} / \mathrm{px}$ at $800 \AA$ \\
\hline \multirow{5}{*}{ Spectral scale } & $0.95 \mathrm{arcsec} / \mathrm{px}$ at $1600 \AA$ \\
\hline & $22.3 \mathrm{m \AA} / \mathrm{px}$ at $500 \AA$ (2nd order $)^{1}$ \\
\hline & $21.0 \mathrm{~m} \AA / \mathrm{px}$ at $800 \AA$ \\
\hline & $45.2 \mathrm{~m} \AA / \mathrm{px}$ at $800 \AA$ (1st order $)$ \\
\hline & $41.9 \mathrm{~m} \AA / \mathrm{px}$ at $1600 \AA$ \\
\hline
\end{tabular}

$34.97^{\circ}$, the wavelength range thus extends from $777 \AA$ to $1613 \AA$ in the 1 st order and from $389 \AA$ to $807 \AA$ in the 2 nd order for the detector A, while from $658 \AA$ to $1502 \AA$ in the 1 st order and from $329 \AA$ to $751 \AA$ in the 2 nd order for the detector B. However, the use of the full range is severely restricted on the short wavelength side by the falloff of the reflectivity of $\mathrm{SiC}$ below $500 \AA$. At the long wavelength end, the range might be restricted by the total count-rate limitations of the detectors. It should be noted that both detectors will be illuminated simultaneously by the grating spectrum in 1st and 2nd orders. A change of detectors thus requires only detector power switching and no optical rearrangement.

At a constant $\theta$, the resolving power of the instrument related to the wavelength can be 
derived by differentiating Eq. 3.1 and combining it with Eq. 3.2.

$$
\frac{\lambda}{\delta \lambda}=\frac{m f_{\lambda} \lambda}{d \cos ^{3} \alpha \Delta_{p x}} .
$$

Hence, the resolving power can be determined at any wavelength. It ranges from 17700 to 38300 for the SUMER grating.

Special attention must be payed to the differences in the definition between the SUMER coordinate system and SOHO inter-instrument coordinate system. ESA defined the SOHO coordinate system $\left(X_{i i}, Y_{i i}\right)$ with $+X_{i i}$ directed towards West of the Sun and $+Y_{i i}$ towards to North of the Sun. In the case of the SUMER instrument, the $+Y$ is defined towards the West and the $+Z$ towards the South (SUMER is isostatically mounted on SOHO with head down). Moreover, the SUMER detectors require an additional coordinate system. The spatial direction will be defined by $Y_{D}=-Y_{i i}$ and the spectral direction by $X_{D}=$ $-X_{i i}$, i.e., in Figure 3.3 , the upper side of the detector with high pixel address is pointing to the South of the Sun.

\subsubsection{Calibrations and corrections}

All SUMER raw data are neither calibrated nor converted to physical units. Various shortcomings caused by the hardware of the instrument need to be corrected. The SUMER team has written various software to make such calibrations and corrections. Here we describe briefly such procedures.

\section{Decompression and reversion}

In order to reduce the telemetry load, the intensities of the image data array have been compressed on-board with different compression methods (usually Method 5 is used). A decompression is thus necessary for the IDL-restore files. Moreover, on the detector, the highest wavelength is on pixel 0 and the lowest on pixel 1023 (cf., Figure 3.3). Therefore, the wavelengths are stored in SUMER image data by descending from left to right. The spectral dimension of the image data must first be reversed for compatibility with the SOHO conventions and other correction routines. In addition, SUMER is fixed "head down" on SOHO, so what we see "up" on our screen is south on the Sun. The spatial dimension of the image data should be also reversed. It should be mentioned that these three procedures should not be applied to the FITS files, which have been decompressed and reversed to "normal" order during their creation.

\section{Flat-field correction}

Non-uniformities in the sensitivity of the detector are caused by the non-linearities of the photocathodes and the microchannel plate. Such non-uniformities are on scale of about 20 pixels or less. Moreover, an odd-even variation (odd-even pattern) in the spatial dimension is caused by a non-linearity of the analogue-to-digital converter (ADC). A flat-field correction should be applied either on board or on the ground for intensity measurements. 
The flat-field data are being accumulated during a three-hour exposure in the H I Lyman continuum between $860 \AA-900 \AA$ while the spectrometer grating is defocused. This provides a deep, although not entirely 'flat', exposure from which the SUMER processor extracts all those features which are smaller than 20 pixels. The small-scale variations amount to as much as $50 \%$. The flat-field pattern changes slightly with time due to the change of the channel plate gain while being used. Therefore the flat-field data need to be updated quite frequently.

\section{Geometrical correction}

The images produced by SUMER is geometrically distorted due to the electronic design of the detector. Moreover, the spectral lines may be inclined with respect to the detector vertical lines, which is caused by the deviation in the orientation of the grating and the detector. Therefore, a geometrical correction must be applied in order to remove the curvature of the lines and put the position of the line profile on a correct spectral pixel. A standard routine for the correction of such geometrical distortion of the image and inclination of lines was developed by T. Moran. The geometrical correction works as follows (Peter and Judge, 1999). The chromospheric O I $1152 \AA$ line was placed at 60 different spectral positions with an interval of about 15 pixels. The telescope was defocused, and thus all spatial structures were smeared out. The resulting variations of the line position should be essentially instrumental. As an ideal case, the central position of the O I line should be at the same spectral pixel along the spatial direction, i.e., it is straight. One can thus correct for the image distortion using the observed $\mathrm{O}$ I displacements interpolated in spectral and spatial direction.

\section{Displacement correction}

The spectrum may be inclined with respect to the detector horizontal lines, which is caused by the deviation in the orientation of the grating and the detector. In this case, the spectral lines may be displaced higher or lower on the detector, varying with the wavelength. On the other hand, the non-linearity of the grating focus mechanism, which is adjusted simultaneously with the wavelength scan, causes an additional shift of the slit image on the detector. A correction for such a vertical displacement of the slit image is necessary for co-registration of images obtained in different reference wavelengths.

\section{Dead time correction}

A correction for the electronic dead-time effect must be applied when the total count rate on the detector during one exposure is above 50000 counts per second. The total count rate can be evaluated through the readout of the X-event and Y-event parameters from the header of the IDL-restore files.

\section{Local gain correction}

For some very strong lines, the local count rate may be so high that the reduced detecting efficiency causes a local gain depression of the detector channel plates. Therefore, a performance of the gain depression correction is necessary in this case.

\section{Line broadening correction}


The instrumental broadening has an additional contribution to the width of spectral lines. This instrumental broadening is slit-dependent and can be taken out by using a deconvolution matrix calculated by P. Lemaire, which gives the deconvolved values for the different slits at different wavelengths for both detectors.

\section{Radiometric calibration}

As we have discussed in Section 3.2.2, the detecting efficiency will vary greatly with different portions, i.e., $\mathrm{KBr}$, bare MCP and Lyman- $\alpha$ attenuators, on the detector and also with different wavelengths. The final output of the solar radiation will also be affected when it travels through the entrance aperture, the primary mirror, the slit, etc. The total spectral responsivities of both detectors as a function of wavelengths and for different portions of the detector are shown in Figure 3.4. In addition, the vertical extension of the long slit image on the detector corresponds to $300^{\prime \prime}$ on the Sun, but is generally not 300 pixels long. It rather is a function of wavelength given by the focal position of the grating which determines the magnification factor of the spectrometer. This is also considered by the radiometric calibration procedure. On the other hand, the detected intensity is given in counts per pixel per sampling interval. A radiometric calibration converts these counts to physical units.

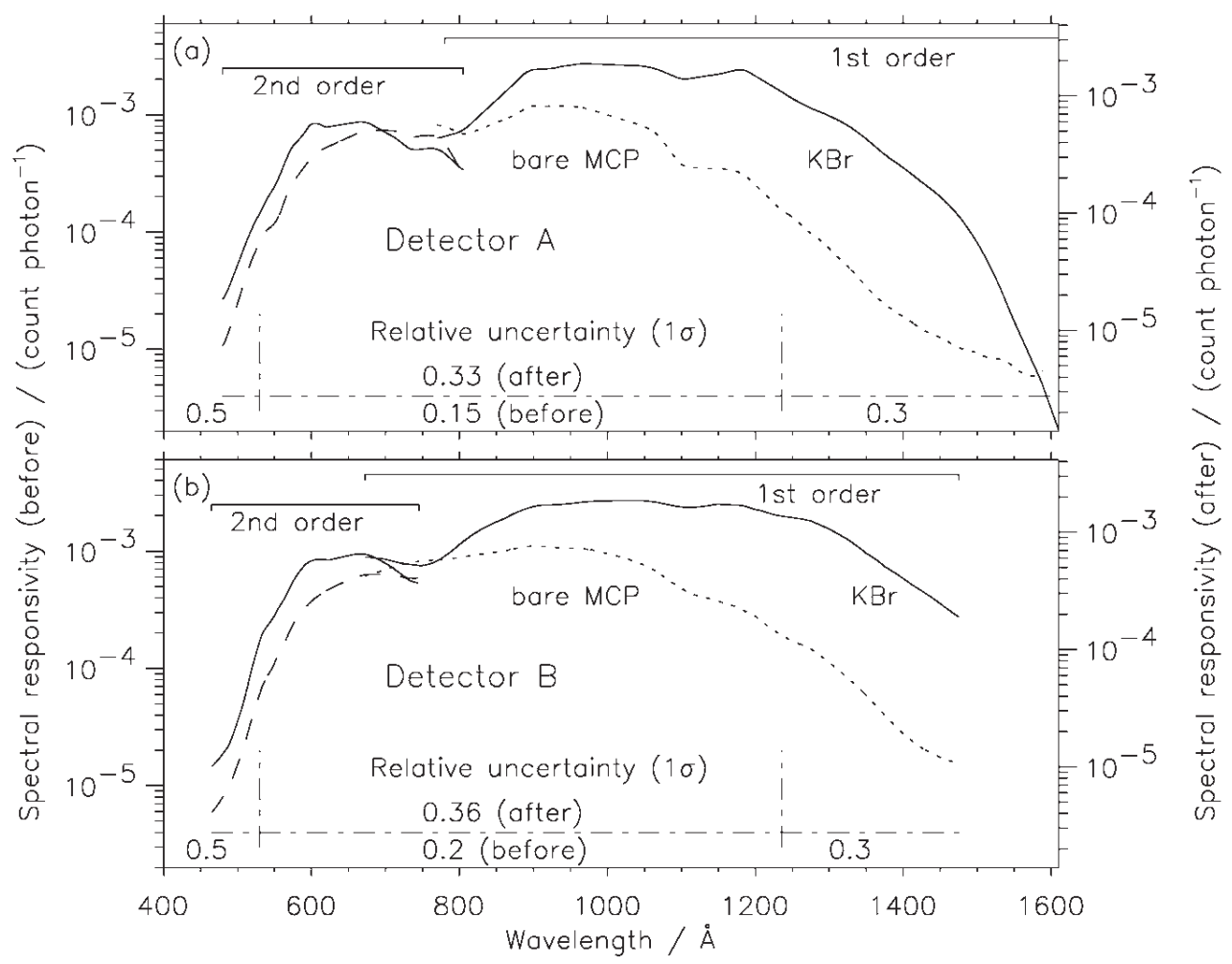

Figure 3.4: The sensitivities of SUMER as obtained during the radiometric calibration. From Wilhelm (2003) 


\subsection{Additional instruments}

\subsubsection{EIT instrument}

The Extreme-ultraviolet Imaging Telescope (EIT) obtains high resolution solar images in different narrow EUV wavelength bands with widths of $6 \AA-10 \AA$. The emission lines are Fe IX $171 \AA$, Fe XII $195 \AA$, Fe XV $284 \AA$ and He II $304 \AA$. Multilayer reflecting coatings deposited on the four quadrants of the telescope mirrors are designed for these 4 different bands. A rotatable mask can be used to select the quadrant illuminated by the Sun. EIT is equipped with a $1024 \times 1024$ CCD camera. Each pixel size corresponds to $2.6^{\prime \prime}$. The EIT full-disk images can thus provide the morphological context of the spectral observations by SUMER and CDS. The detailed description of this instrument was given by Delaboudinière et al. (1995).

\subsubsection{MDI instrument}

The Michelson Doppler Imager (MDI) obtains high precision solar images $(1024 \times 1024$ pixels) of the line-of-sight velocity, line intensity, continuum intensity and longitudinal magnetic field components by sampling the Ni I $6768 \AA$ line with a wide-field tunable Michelson interferometer. It can be operated in a full-disk mode ( 2 " equivalent pixel size) to resolve modes in the range of harmonic degree $3 \leq l \leq 1500$ or in a high-resolution mode $\left(0.65^{\prime \prime}\right.$ per pixel size) to resolve modes as high as $l=4500$. The high-resolution fieldof-view is roughly 650 " squared and is centred about 160 " north of the equator on the central meridian. We will use the magnetic field data, which were processed to "Level 1.5 " (calibrated) by the MDI team (http://soi.stanford.edu/sssc). The detailed description of this instrument was given by Scherrer et al. (1995).

\subsubsection{NASA/NSO Spectromagnetograph}

The NASA/NSO spectromagnetograph is a new focal plane instrument built in early 1990's for use at the National Solar Observatory/Kitt Peak (NSO/KP) Vacuum Telescope. The widths, depths and wavelength positions of line profiles can be deduced directly with spectral and spatial resolutions. The magnetograms are computed as realtime processes from the raw data. The rms noise per pixel is estimated to be $4 \mathrm{G}$ at the disk center, by making an observation with the polarized light modulator switched off. However, the accuracy of measured fields is usually limited by atmospheric seeing rather than instrument system noise. This might cause an uncertainty of order $10 \%$ of the measured field strength (J. Harvey, 2002, private communication). A detailed description of the measurement has been given in Jones et al. (1992). In this thesis, the photospheric magnetogram was obtained by anonymous ftp. The used Fe I ( $8688 \AA$ ) magnetograms have a spatial resolution element of $1.14 \times 1.14 \operatorname{arcsec}^{2}$. Following the documentation of the field data, we have 
multiplied the instrumental values by a factor of 1.46 , thus converting them to magnetic field strength given in Gauss.

\subsubsection{SWE instrument}

The Solar Wind Experiment (SWE) instrument on WIND spacecraft was designed to study outstanding problems in solar wind physics (Ogilvie et al., 1995). The "key parameters", such as velocity, density, and temperature of the solar wind are measured by Faraday cup sensors. The proton velocity and number density, which will be used in this thesis, are measured with precisions of about $\pm 3 \%$ and $\pm 10 \%$, respectively.

\subsubsection{MFI instrument}

The Magnetic Field Investigation (MFI) instrument is also on board WIND spacecraft and provides 3-component measurements of the interplanetary magnetic field (IMF) (Lepping et al. (1995). This instrument is based on a boom-mounted dual tri-axial fluxgate magnetometer system. The instrument has a measurement accuracy of less than $0.08 \mathrm{nT}$ and a precision of about $\pm 0.025 \%$.

\subsection{Diagnostic methods with FUV/EUV lines}

Spectroscopic diagnostics in FUV/EUV are powerful tools used to probe the upper solar atmosphere (upper chromosphere, transition region and corona). A detailed discussion of diagnostic methods has been given in the reviews contributed by Mariska (1992) and Mason and Monsignori Fossi (1994). Here, a brief description is given to introduce the basic knowledge that is useful for this thesis.

\subsubsection{Atomic processes in the upper solar atmosphere}

As we mentioned in the introduction, the upper solar atmosphere contains very hot (between ten thousand $\mathrm{K}$ and a few million $\mathrm{K}$ ) and low-density (lower than $10^{11} \mathrm{~cm}^{-3}$ ) plasma. Most atoms are fully ionized under this condition. Atomic processes that should be considered are excitation and de-excitation, that change the energy state of each ion, as well as ionization and recombination, that change the charge state of each atom. Essentially, these processes all result from electronic transitions between different energy states. From atomic physics, we know that there are three types of radiative transitions, namely, bound-bound, free-bound and free-free. The bound-bound transition is responsible for the formation of emission and absorption lines; the free-bound transition results in the process of ionization and recombination, as well as the formation of continuum emission. Concerning the characteristic times involved, the process of excitation balance is much 
faster than that of ionization balance in the low-density plasma (see detailed discussion in Mariska, 1992) of the solar atmosphere.

\section{Excitation and de-excitation}

In the upper atmosphere of the quiet Sun, the main cause of producing excited states is collisional process. By colliding with the electron, an atom or ion with a charge state $X^{m+}$ can be excited from a lower energy state $i$ to a higher one $j$ :

$$
X_{i}^{m+}+e \Rightarrow X_{j}^{m+}+e^{\prime}
$$

During the de-excitation process, the spontaneous radiative decay is very efficient for allowed transitions. However, the collisional de-excitation becomes very important for forbidden and intercombination transitions from metastable levels, in which case the spontaneous transition probability is smaller. This is very useful for electron density diagnostics, which we will discuss in a later section. These processes of de-excitation are:

$$
\begin{gathered}
X_{j}^{m+} \Rightarrow X_{i}^{m+}+h \nu_{j i}, \\
X_{j}^{m+}+e \Rightarrow X_{i}^{m+}+e^{\prime},
\end{gathered}
$$

where $h \nu_{j i}$ is the energy of the photon emitted during the transition.

Collisional excitation and de-excitation by protons may also be important. The wavelengths of the emission lines that are produced by this process range in the visible and infrared portions of the spectrum.

If only the above processes of excitation and de-excitation are considered, the rate equation concerning the balance of these two processes (for each level denoted as $i$ ) is given by

$$
\sum_{j \neq i} n_{j} n_{e} C_{j i}-n_{i} \sum_{j \neq i} n_{e} C_{i j}+\sum_{j>i} n_{j} A_{j i}-n_{i} \sum_{j<i} A_{i j}=0
$$

where $C_{i j}$ and $C_{j i}$ represent collisional rate coefficients, and $A_{i j}$ and $A_{j i}$ spontaneous radiation transition probabilities. Here a steady state is assumed because of the short characteristic time of these processes compared with the processes of ionization and recombination.

\section{Ionization and recombination}

In the upper solar atmosphere, the process of ionization is dominated by collisional interactions, which is induced either by direct electron impact or by autoionization following a collisional excitation. Photoionization is negligible because the radiation field is too weak there. The important processes of recombination include radiative recombination and dielectronic recombination. At low temperatures $\left(10^{4} \mathrm{~K}-10^{5} \mathrm{~K}\right)$, a charge exchange with 
$\mathrm{H}$ or He may also be important for the recombination process. These processes can be summarized as follows:

$$
\begin{gathered}
X_{i}^{m+}+e \Rightarrow X_{j}^{(m+1)+}+e+e^{\prime} \text { (direct impact ionization), } \\
X_{i}^{(m+1)+}+e \Rightarrow\left(X_{j}^{m+}\right)^{* *} \Rightarrow X_{k}^{(m+1)+}+e^{\prime} \text { (autoionization), } \\
X_{i}^{(m+1)+}+e \Rightarrow X_{j}^{m+}+h \nu \text { (radiative recombination), } \\
X_{i}^{(m+1)+}+e \Rightarrow\left(X_{j}^{m+}\right)^{* *} \Rightarrow X_{k}^{m+}+h \nu \text { (dielectronic recombination), } \\
X_{i}^{(m+1)+}+Y \Rightarrow X_{j}^{m+}+Y^{+}+\Delta E \text { (charge exchange), }
\end{gathered}
$$

where $\left(X_{j}^{m+}\right)^{* *}$ indicates a doubly excited state of the ion $X_{j}^{m+}$, which is formed when a free electron is captured by the ion with charge $(m+1)+$. This newly formed ion can then either autoionize back to the next degree of ionization by interacting with the adjacent continuum state or simply decay radiatively to another bound state below the ionization threshold.

\subsubsection{Formation of line and continuum emission}

\section{Emission lines}

The process that produces emission lines is called bound-bound transition. The resulting spectral lines can be grouped into three categories according to the different transition process: allowed, intercombination and forbidden lines. Allowed lines are produced by electric dipole transitions without change of the electronic spin. In this case, collisional excitations from the ground level or a metastable level are immediately followed by spontaneous radiative decays having large transition probabilities. Intercombination and forbidden lines involve spin changes and originate from metastable levels, for which the transition probabilities are much smaller than for allowed transitions. These two types of lines can only be observed in a low-density plasma (e.g., in the corona), where the collisional effect is so weak that metastable levels will not be depopulated by collisions before they decay radiatively.

\section{Continuum emission}

Continuum emission in FUV/EUV is produced by free-free, free-bound and two-photon transitions. A free-free transition takes place when a free electron interacts with a charged particle. In this process, the electron jumps down from a higher energy state to a lower one and a photon is simultaneously released. For a Maxwellian velocity distribution of the electrons, this process is also called thermal bremsstrahlung. A free-bound transition is also known as radiative recombination, which is induced when a free electron is captured by an ion into a bound state. The charge state of the element is simultaneously changed 
in this process. The two-photon continuum is produced by simultaneous emission of two photons from $\mathrm{H}$ - and He-like ions in the metastable 2s level, and may be important when $T \leq 3 \times 10^{4} \mathrm{~K}$ (see, Mason and Monsignori Fossi, 1994, and references therein).

\subsubsection{Diagnostics with FUV/EUV lines}

\section{Emissivity calculation}

According to the above discussion, an atom or ion in an excited state can be deexcited from an upper energy level to a lower one by a spontaneous radiative decay, and emit a photon of energy $h \nu_{j i}$. The volume emissivity of the plasma in the $j$ to $i$ transition is:

$$
\varepsilon_{j i}=h \nu_{j i} A_{j i} n_{j}=\frac{h c}{\lambda_{j i}} A_{j i} n_{j}\left(\operatorname{erg~cm}^{-3} \mathrm{~s}^{-1}\right)
$$

where $n_{j}$ is number density of the level $j$ of the ion; $A_{j i}$ is the Einstein spontaneous emission coefficient.

The assumption that the observed FUV/EUV spectral lines are emitted in the optically thin plasma is valid in the upper solar atmosphere, so that absorption can be neglected. Then the radiative flux observed at a certain distance $R$ from the Sun for an emission line with wavelength $\lambda_{j i}$ is given by

$$
F_{j i}=\frac{1}{4 \pi R^{2}} \int_{\Delta V} \varepsilon_{j i} d V\left(\operatorname{erg~cm}^{-2} \mathrm{~s}^{-1}\right)
$$

where $\Delta V$ is the volume of plasma along the path defined between the observer and object on the Sun.

For the multi-component solar atmosphere, the number density of ions can be expressed in terms of ratios of other parameters of the plasma:

$$
n_{j}=\frac{n_{j}}{n_{\text {ion }}} \frac{n_{\text {ion }}}{n_{\text {el }}} \frac{n_{e l}}{n_{H}} \frac{n_{H}}{n_{e}} n_{e},
$$

where $n_{i} / n_{\text {ion }}$ is the population of excited level $j$ relative to the total number density of an ion; $n_{i o n} / n_{e l}$ is the ionization ratio of the ion; $n_{e l} / n_{H}$ is the abundance of the element relative to hydrogen, which is usually denoted as $A_{e l} ; n_{H} / n_{e}$ is the number density of hydrogen relative to the number density of the electron. Thus, the equation 3.14 can be re-written as

$$
\varepsilon_{j i}=\frac{h c}{\lambda_{j i}} A_{j i} \frac{n_{j}}{n_{\text {ion }}} \frac{n_{\text {ion }}}{n_{e l}} A_{e l} \frac{n_{H}}{n_{e}} n_{e}
$$

In a simple two-level approximation for each transition, in which collisional excitations from the ground level balance spontaneous radiative decays from the excited levels, the 
population of an upper level $j$ is negligible compared with that of the ground level $g$, i.e., $n_{g} \approx n_{\text {ion }}$. Thus the statistical equilibrium equation 3.8 can be written as

$$
n_{e} n_{g} C_{g j}=n_{j} \sum_{k<j} A_{j k} .
$$

The volume emissivity of the plasma in the $j$ to $g$ transition is

$$
\varepsilon_{j g}=\frac{h c}{\lambda_{j g}} \frac{A_{j g}}{\sum_{k<j} A_{j k}} C_{g j} \frac{n_{i o n}}{n_{e l}} A_{e l} \frac{n_{H}}{n_{e}} n_{e}^{2} .
$$

A contribution function $G(T)$ is defined by

$$
G(T)=\frac{A_{j g}}{\sum_{k<j} A_{j k}} C_{g j} \frac{n_{i o n}}{n_{e l}},
$$

which is strongly dependent on temperature $T$. Then the volume emissivity can be expressed in the form

$$
\varepsilon_{j g}=\frac{h c}{\lambda_{j g}} A_{e l} \frac{n_{H}}{n_{e}} G(T) n_{e}^{2} .
$$

We may assume that the abundance of the element relative to hydrogen $A_{e l}$ and the abundance of hydrogen relative to the electron density $\frac{n_{H}}{n_{e}}$ are independent of the plasma volume. The flux equation 3.15 can thus be rewritten as

$$
F_{j g}=\frac{1}{4 \pi R^{2}} \frac{h c}{\lambda_{j g}} A_{e l} \frac{n_{H}}{n_{e}} \int_{\Delta V} G(T) n_{e}^{2} d V
$$

where the volume integral is referred to as differential emission measure (DEM), quantifying the amount of material emitting at temperature $T$.

\section{Line profiles}

If the ions are in local thermal equilibrium (LTE), they have a Maxwellian velocity distribution. Therefore, the frequency (wavelength) of the emitted photons has a corresponding distribution because of the Doppler effect. Consequently, the spectral line will be broadened. In addition, the bulk velocity of the ions will result in deviation of the line central position from the one expected at rest, and possible wave and turbulent motions will lead to an additional broadening of the spectral line. Analysis of the line profile becomes very important for deducing the detailed physical properties of the plasma.

Considering an ion has a velocity component $v$ along the line of sight (LOS) (towards the observer is positive), the observed frequency of the emitted photon will be

$$
\nu=\nu_{0} \frac{c}{c-v}
$$


where $\nu_{0}$ is the rest frequency of the photon. If $v \ll c$, the relation between $v$ and other parameters is given by

$$
\begin{gathered}
v \simeq c \frac{\nu-\nu_{0}}{\nu_{0}}, \\
d v=\frac{c}{\nu_{0}} d \nu .
\end{gathered}
$$

According to the Maxwellian velocity distribution, the number of ions in a velocity range from $v$ to $v+d v$ relative to the total number of ions is

$$
\frac{d n}{n}=\sqrt{\frac{m}{2 \pi k T}} \exp \left(-\frac{m v^{2}}{2 k T}\right) d v
$$

Here $m$ is the mass of the ion; $k$ is Boltzman's constant; $T$ is the temperature. Thus the frequency profile function $\psi_{\nu}$ can be obtained, by integrating the equation 3.26 , to be

$$
\psi_{\nu}=\frac{1}{\sqrt{\pi} \Delta \nu_{D}} \exp \left(-\frac{\left(\nu-\nu_{0}\right)^{2}}{\Delta \nu_{D}^{2}}\right) .
$$

It shows that the profile has a shape with Gaussian form. Here $\Delta \nu_{D}$ is the Doppler width, which is expressed by

$$
\Delta \nu_{D}=\frac{\nu_{0}}{c} \sqrt{\frac{2 k T}{m}}
$$

or in terms of wavelength as

$$
\Delta \lambda_{D}=\frac{\lambda_{0}}{c} \sqrt{\frac{2 k T}{m}} .
$$

Another frequently used definition of the line width is the full width at half maximum (FWHM), which is obtained as

$$
\Delta \lambda_{1 / 2}=2 \sqrt{\ln 2} \Delta \lambda_{D}
$$

Actually, some other mechanisms may contribute additional broadenings to the line profile observed in the upper solar atmosphere, among which are instrumental broadening and non-thermal motions. The latter may possibly result from wave and turbulent motions. Including them, the Doppler width is re-written as

$$
\Delta \nu_{D}=\frac{\nu_{0}}{c} \sqrt{\frac{2 k T}{m}+v_{u}^{2}+\sigma_{I}^{2}} .
$$

Here $v_{u}$ is the most probable non-thermal speed, and $\sigma_{I}$ is the equivalent speed contributed by instrumental broadening. 


\section{Diagnostic of electron density}

The existence of metastable levels for an atom can be used to measure the electron density. According to the previous discussion, the spontaneous transition probability is very small for intercombination and forbidden transitions from metastable levels, so that the collisional de-excitation should be taken into account in the process of population and depopulation of such levels if the electron density is large enough. Moreover, the depopulation rate caused by collisions will increase with increasing electron density, and at the same time, reduce the radiative rate by spontaneous transitions.

A three-level atomic configuration is given here as an example to show how the electron density can be deduced from the intensity ratio between a forbidden line and an allowed line. We denote $g$ as the ground level, 1 as the first excited level and 2 as the second excited level. We assume the level 1 is allowed, and thus it will always be populated by collisions and depopulated by the radiative decay. Level 2 is metastable and populated only by collisions and depopulated by both the radiative decay and collisional de-excitation. Therefore we write the excitation rate equation 3.8 for levels 1 and 2 as

$$
n_{g} n_{e} C_{g 2}-n_{2} n_{e} C_{2 g}-n_{2} n_{e} C_{21}-n_{2} A_{21}-n_{2} A_{2 g}=0
$$

and

$$
n_{g} n_{e} C_{g 1}+n_{2} n_{e} C_{21}+n_{2} A_{21}-n_{1} A_{1 g}=0 .
$$

The line ratio $R$ of the forbidden transition from 2 to $g$ and the allowed transition from 1 to $g$ can be obtained by combining these two equations

$$
R=\frac{n_{2} A_{2 g}}{n_{1} A_{1 g}}=\frac{A_{2 g}}{\frac{C_{g 1}}{C_{g 2}}\left(A_{2 g}+n_{e} C_{2 g}\right)+\frac{C_{g 1}+C_{g 2}}{C_{g 2}}\left(A_{21}+n_{e} C_{21}\right)} .
$$

In case that the electron density is very low, collisional effects will be very weak and can be neglected $\left(A \gg n_{e} C\right)$. Then this equation can be simplified to

$$
R=\frac{A_{2 g}}{\frac{C_{g 1}}{C_{g 2}} A_{2 g}+\frac{C_{g 1}+C_{g 2}}{C_{g 2}} A_{21}} .
$$

Since we have $A_{21} \ll A_{2 g} \ll A_{1 g}$, the equation can be simplified further to read

$$
R=\frac{C_{g 2}}{C_{g 1}}
$$

If the electron density becomes so high that the collisional depopulation dominates $(A \ll$ $\left.n_{e} C\right)$, then we have

$$
R=\frac{A_{2 g}}{n_{e}\left[\frac{C_{g 1}}{C_{g 2}} C_{2 g}+\frac{C_{g 1}+C_{g 2}}{C_{g 2}} C_{21}\right]} \propto \frac{1}{n_{e}} .
$$

The line ratio is now density sensitive. It can be used to measure the electron density if the intensities of the two lines can be determined by observations. It should be noted that this line ratio is weakly dependent on the temperature, because the collision rate is a function of the temperature. 


\section{Diagnostic of electron temperature}

If we assume that the plasma is in ionization equilibrium and isothermal, then the plasma temperature can simply be deduced by the assumption that the temperature corresponds to the temperature of the maximum value of the contribution function $G(T)$. In practice, the usage of ions that are formed over broad temperature ranges, such as Li-like, He-like and H-like ions, should be avoided.

Another method that may be more accurate for determining the electron temperature is to use the intensity ratio of two allowed lines. The two lines are excited from and decay to the ground level, but may have significantly different excitation energy $\left(\left(\Delta E_{g 1}-\Delta E_{g 2}\right) / k T \gg 1\right)$. Considering a three-level system with the ground level $g$ and two excited levels 1 and 2, the intensity ratio is given by

$$
R=\frac{\Delta E_{g 2}}{\Delta E_{g 1}} \frac{C_{g 2}}{C_{g 1}}
$$

Here the collision rate coefficient $C_{i j}$ has the form (Mariska, 1992):

$$
C_{i j}=\frac{8.63 \times 10^{-6} \Omega_{i j}}{\omega_{i} T^{1 / 2}} \exp \left(-\frac{\Delta E_{i j}}{k T}\right)
$$

where $\Omega_{i j}$ is the collision strength and $\omega_{i}$ is the statistical weight of level $i$. Then we have

$$
R=\frac{\Delta E_{g 2}}{\Delta E_{g 1}} \frac{\Omega_{g 2}}{\Omega_{g 1}} \exp \left(\frac{\Delta E_{g 1}-\Delta E_{g 2}}{k T}\right)
$$

Under the condition of $\left(\Delta E_{g 1}-\Delta E_{g 2}\right) / k T \gg 1$, this ratio will be a sensitive function of the electron temperature. 



\section{Chapter 4}

\section{Observations and Methods of Data Analysis}

\subsection{Description of observations}

Shortly after the launch of SOHO, the Sun reached its minimum of activity in 1996. Then the rising phase of the next solar cycle began. During the past sunspot minimum, two persistent large coronal holes of opposite magnetic polarity cover each pole of the Sun, while the low-latitude coronal holes were very rare features. Recent study showed that the first non-polar coronal hole was observed during January and March 1996 (Belenko, 2001). However, with increasing solar activity in the new sunspot cycle, smaller coronal holes formed more and more at lower latitudes.

Compared with the large number of observations by SUMER within polar coronal holes, the data sets obtained in the equatorial coronal holes are relatively few. We searched in the data catalogue of SUMER and found the data sets which may suitable to investigate the morphological or physical properties of ECHs. The collection of these data is listed in Table 4.1. Please note that the collection may not be complete. Among them, those marked by "a" will be analyzed in this thesis. The observed ECHs include two types of low-latitude coronal holes, i.e., the small isolated coronal holes and large "Elephant's Trunk" type coronal hole, which was part of the extension of the polar coronal hole. During the SOHO mission, a well known "Elephant's Trunk" coronal hole was observed during August and October 1996 (CH3), which has been intensively investigated by many authors using different instruments.

In Table 4.2, we also list the data observed with SUMER in a quiet-Sun (QS) region near the disk center. These QS data were obtained during the days 8 and 9 March, 1999, very near the dates when observing the coronal hole ( $\mathrm{CH} 6$ ). Moreover, SUMER was operated under the same instrumental conditions. Therefore, the data can be used for a comparison of the two regions. In addition, the QS data can also be used to make additional geometrical calibration for deducing Dopplergrams (see discussion in the following).

SUMER has the capability of imaging the Sun by moving the spectrometer slit perpendicular to the slit direction. Generally, there are three modes of observing sequence to map the Sun depending on how the slit moves. One mode refers here to "raster scan", where the slit moves with a given step size. With this mode one can map a large area of the Sun. The second mode refers here to "solar rotation tracking", where the compensation of the solar rotation is turned on. With this mode a structure can be traced on the disk following solar rotation. The third mode refers here to "fixed slit", where the slit does not move and the scan is completed by exploiting solar rotation. In our data, all three modes have been applied in different observations. 


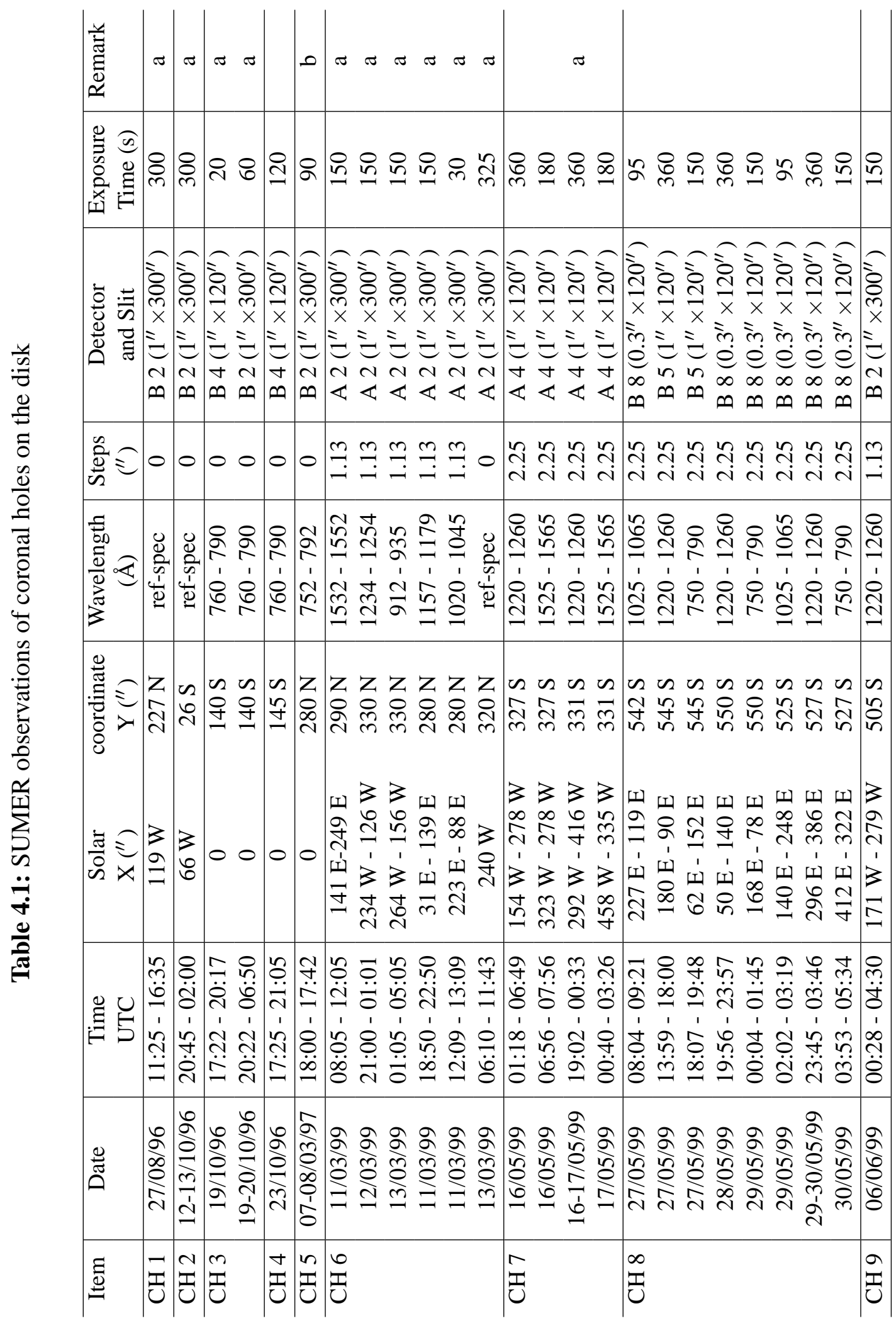




\begin{tabular}{|c|c|c|c|c|}
\hline 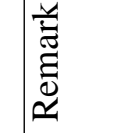 & 0 & $\begin{array}{llllll}0 & 0 & \pi & \pi\end{array}$ & $\sigma$ & 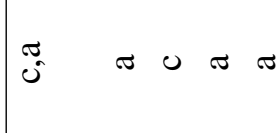 \\
\hline 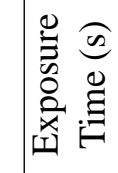 & 요 & 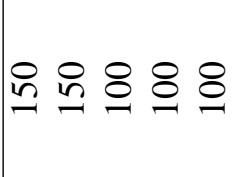 & 8 & 은 요 요 \\
\hline 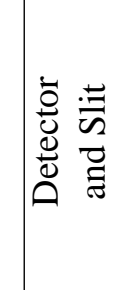 & 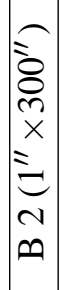 & 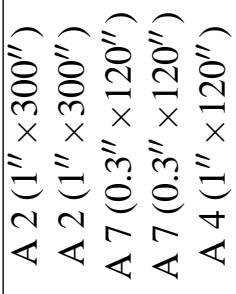 & 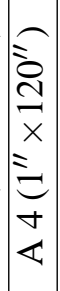 & 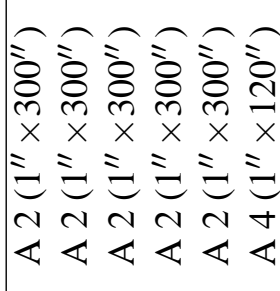 \\
\hline$\stackrel{0}{\frac{n}{\omega}} \cong$ & $\begin{array}{l}\infty \\
\infty \\
- \\
-i\end{array}$ & 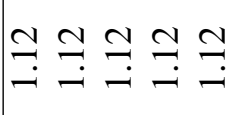 & $\stackrel{\simeq}{\beth}$ & 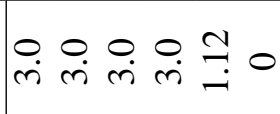 \\
\hline 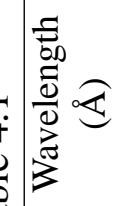 & 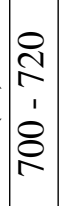 & 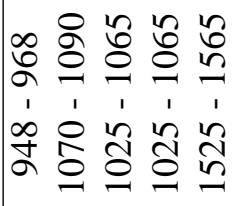 & $\begin{array}{l}n \\
n \\
n \\
1 \\
2 \\
0 \\
n \\
n\end{array}$ & 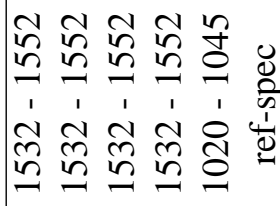 \\
\hline 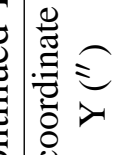 & $\begin{array}{l}n \\
0 \\
\mathbb{N}\end{array}$ & 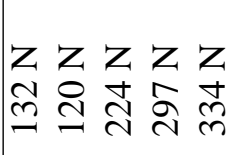 & $\begin{array}{l}Z \\
n \\
\infty \\
+\end{array}$ & 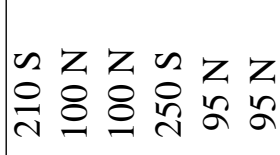 \\
\hline 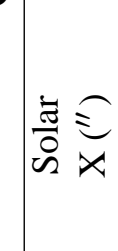 & $\begin{array}{l}3 \\
\overline{2} \\
2 \\
1 \\
\pm \\
= \\
=\end{array}$ & 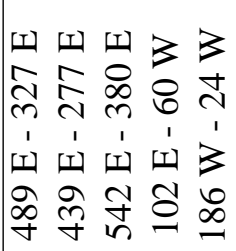 & \begin{tabular}{l|}
3 \\
\\
\pm \\
1 \\
3 \\
0 \\
0 \\
0
\end{tabular} & 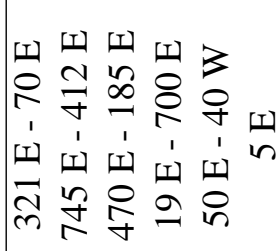 \\
\hline 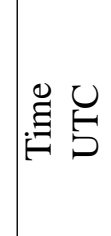 & $\begin{array}{c}\dot{r} \\
\ddot{n} \\
\sim \\
1 \\
\dot{0} \\
\dot{\infty} \\
0\end{array}$ & 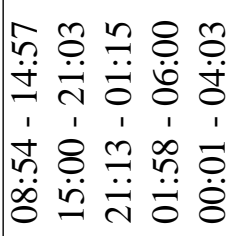 & $\begin{array}{c}0 \\
\dot{\leftrightarrow} \\
0 \\
1 \\
\dot{0} \\
\dot{0}\end{array}$ & 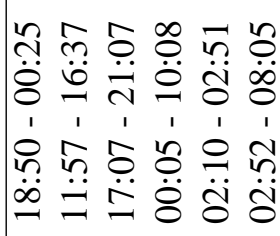 \\
\hline 节 & 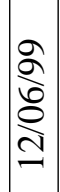 & 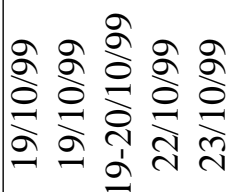 & 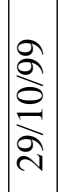 & 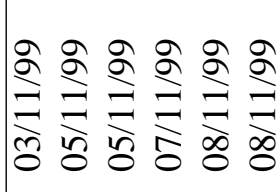 \\
\hline 胥 & $\begin{array}{l}0 \\
\underline{\pi} \\
\underline{U}\end{array}$ & 全 & 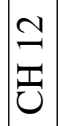 & $\frac{n}{\tilde{I}}$ \\
\hline
\end{tabular}

\begin{tabular}{|c|c|}
\hline 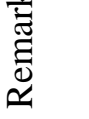 & $\theta \sigma \theta \sigma \sigma \theta$ \\
\hline 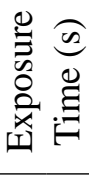 & 은은으요 요 \\
\hline 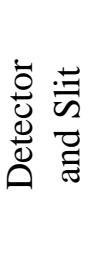 & 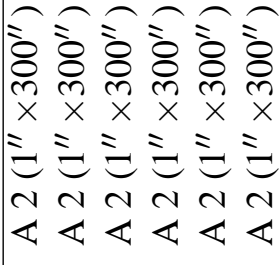 \\
\hline$\stackrel{0}{\frac{0}{2}}=$ & 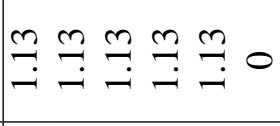 \\
\hline $\begin{array}{l}\frac{5}{5} \\
\frac{0}{0} \\
\frac{0}{0}\end{array}$ & 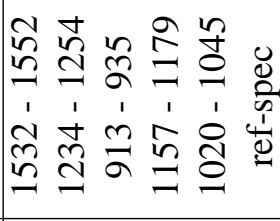 \\
\hline 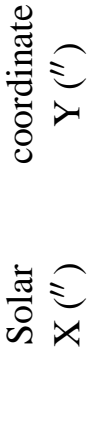 & 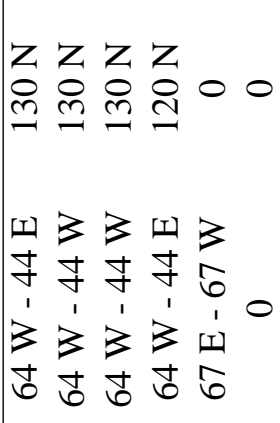 \\
\hline$\stackrel{\Xi}{\Xi}$ & 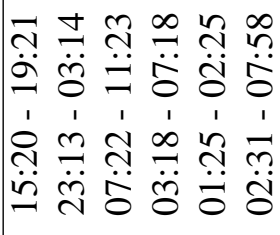 \\
\hline $\begin{array}{l}\stackrel{\mathscr{\pi}}{\pi} \\
\stackrel{0}{\pi}\end{array}$ & 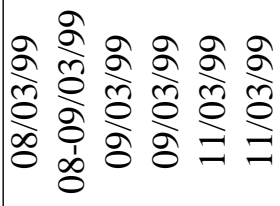 \\
\hline$\underset{\Xi}{\Xi}$ & $\overline{2}$ \\
\hline
\end{tabular}


In Table 4.1 and 4.2, the observational parameters of the data sets are given. The date and time are given at the start and the end of the observation. The pointing in $\mathrm{X}$ direction (West-East) refers to the slit position at the start and the end of the observation for the raster scan sequence, but only the start position is given for the solar-rotation-tracking and fixed slit sequences. The pointing in Y direction (South-North) refers to the slit center at the start of the observation. It is noted that the "reference spectrum" is a standard observing sequence of SUMER. It consists of a series of full detector readouts at different wavelengths.

\subsection{Identification of coronal holes}

According to the characteristics described in Chapter 2, coronal holes are regions with reduced EUV and X-ray emission in the solar corona. Previous studies have found that the appearances of the coronal hole seen at different wavelengths were significantly different in shape and area (see, e.g., Wilhelm et al., 2002; Bromage et al., 2000). Usually, coronal holes seen in X-ray have a wider and stable shape than in EUV lines. They appear also different in shape seen in different EUV lines with different formation temperatures, e.g., sometimes they are seen prominently in the Fe XII $\left(1.6 \times 10^{6} \mathrm{~K}\right)$ and Fe XV $\left(2 \times 10^{6} \mathrm{~K}\right)$ channels of EIT, but not clearly in the Ne VIII line $\left(6.3 \times 10^{5} \mathrm{~K}\right)$ (Wilhelm et al., 2002). This difference indicates possibly the increasing size of the coronal hole with increasing temperature or height. However, most holes we have observed appear very dark in all these lines. Coronal holes can also be seen in the He I line (10830 $\AA$ ) formed at a temperature of less than 20,000 K (Harvey et al., 1975). The National Solar Observatory at Kitt Peak (NSO/KP) provides coronal hole maps in this line every day.

In our study, because lines with relatively low formation temperatures are analysed and also the field of view (FOV) of SUMER is small, it is difficult to identify coronal holes unambiguously by SUMER data alone. We thus identify coronal holes by using EIT images obtained in the Fe XII channel. This is rather feasible because we can always obtain the EIT images that were observed almost simultaneously as the SUMER images. In addition, coronal hole maps in the He I (10830 ̊̊) line provided by NSO/KP, and magnetic field data observed by MDI and NSO/KP can be used as a verification.

In determining the boundaries of the coronal holes, we first plot the histogram of the Fe XII intensity taken from the dark region in the hole, and thus obtain a peak intensity from this plot. Then we search for a quiet area far from the active region on the disk, repeat the first step, and obtain a second peak intensity. A threshold of the intensity is then determined by taking the mean of the two peak intensities, by help of which the boundary of a coronal hole can be marked by plotting the corresponding contour.

As was discussed above, the boundaries of the coronal holes inferred by different wavelengths are somehow different in shape. However, this should not be a problem because we only focus on studying the main part of the coronal holes rather than their boundaries. 

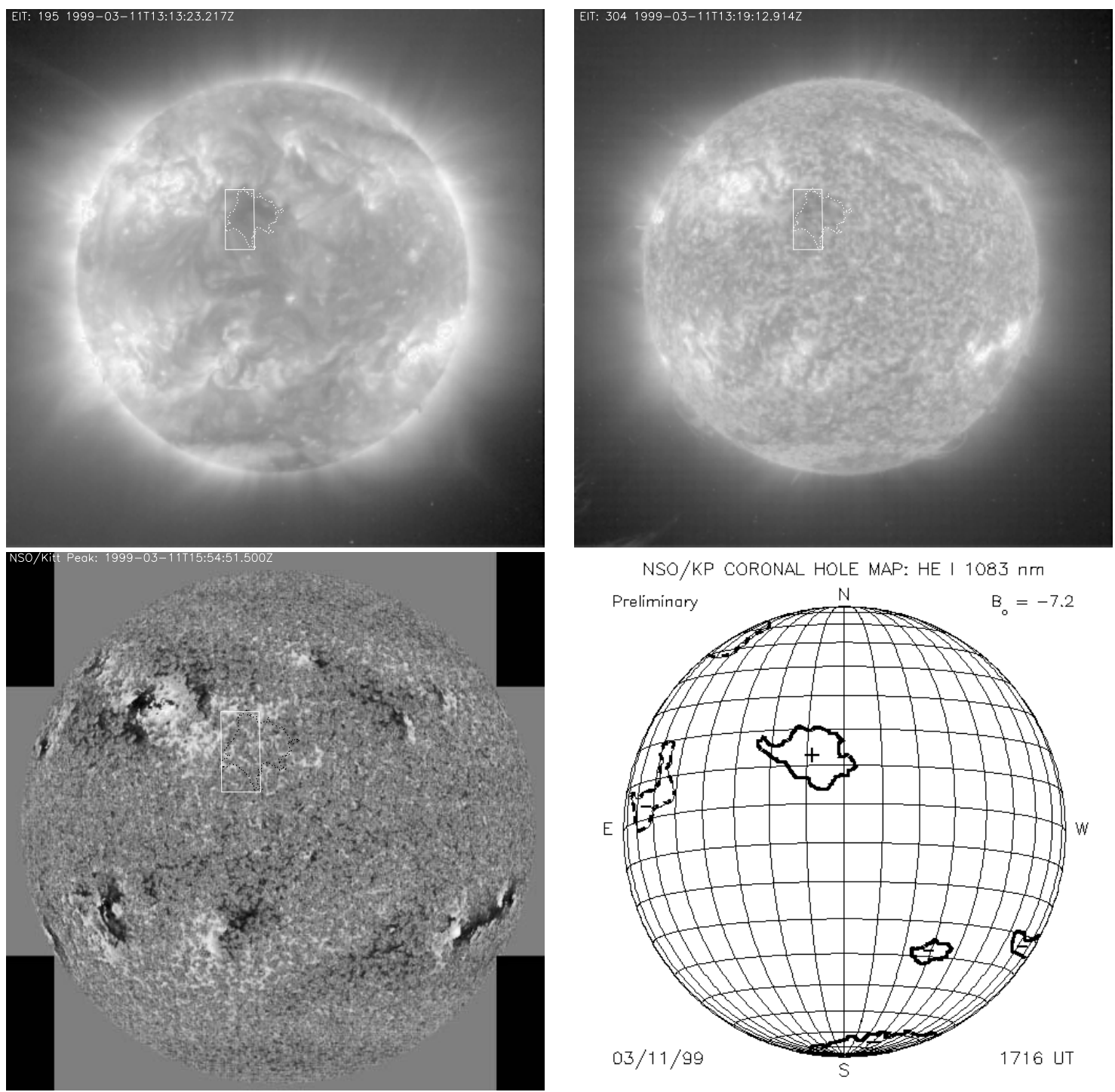

NSO/KP CORONAL HOLE MAP: HE I $1083 \mathrm{~nm}$

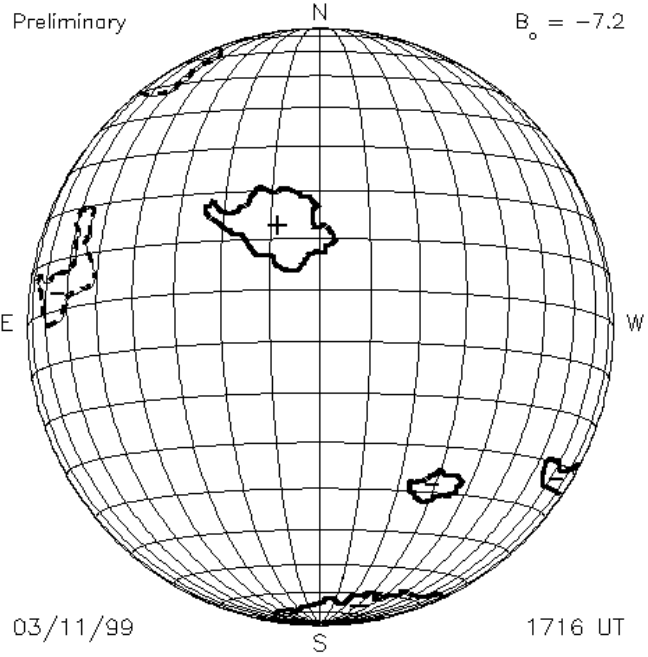

Figure 4.1: Context of the SUMER observation of a coronal hole on the disk on 11 March, 1999 (CH 6 ), superimposed on (a) Fe XII (195 A) (upper left); (b) He II (304 $⿱$ ) (upper right) images taken by EIT at the same day; and (c) the magnetic field image obtained by NSO/KP at the same day. Note that the average magnetic field is about $7.5 \mathrm{G}$ in the coronal hole (bottom left). (d) The Kitt Peak coronal hole map for this ECH (bottom right).

In Figure 4.1, as an example, we show a small ECH observed on the disk by SUMER on 11 March, 1999 (CH6). The FOV of SUMER marked by white box is superimposed on images taken by EIT at Fe XII (195 $\AA$ ) (upper left) and He II (304 $\AA$ ) (upper right) on the same day. These two channels both show as a signature of the coronal hole reduced emission in a small area. The magnetic field image obtained by NSO/KP on the same day is also displayed, again, with a superposition of the FOV of SUMER (bottom left). The magnetic field averaged across the coronal hole is about $7.5 \mathrm{G}$, which indicates that this area is dominated by a unipolar magnetic field. In addition, the NSO/KP coronal hole 

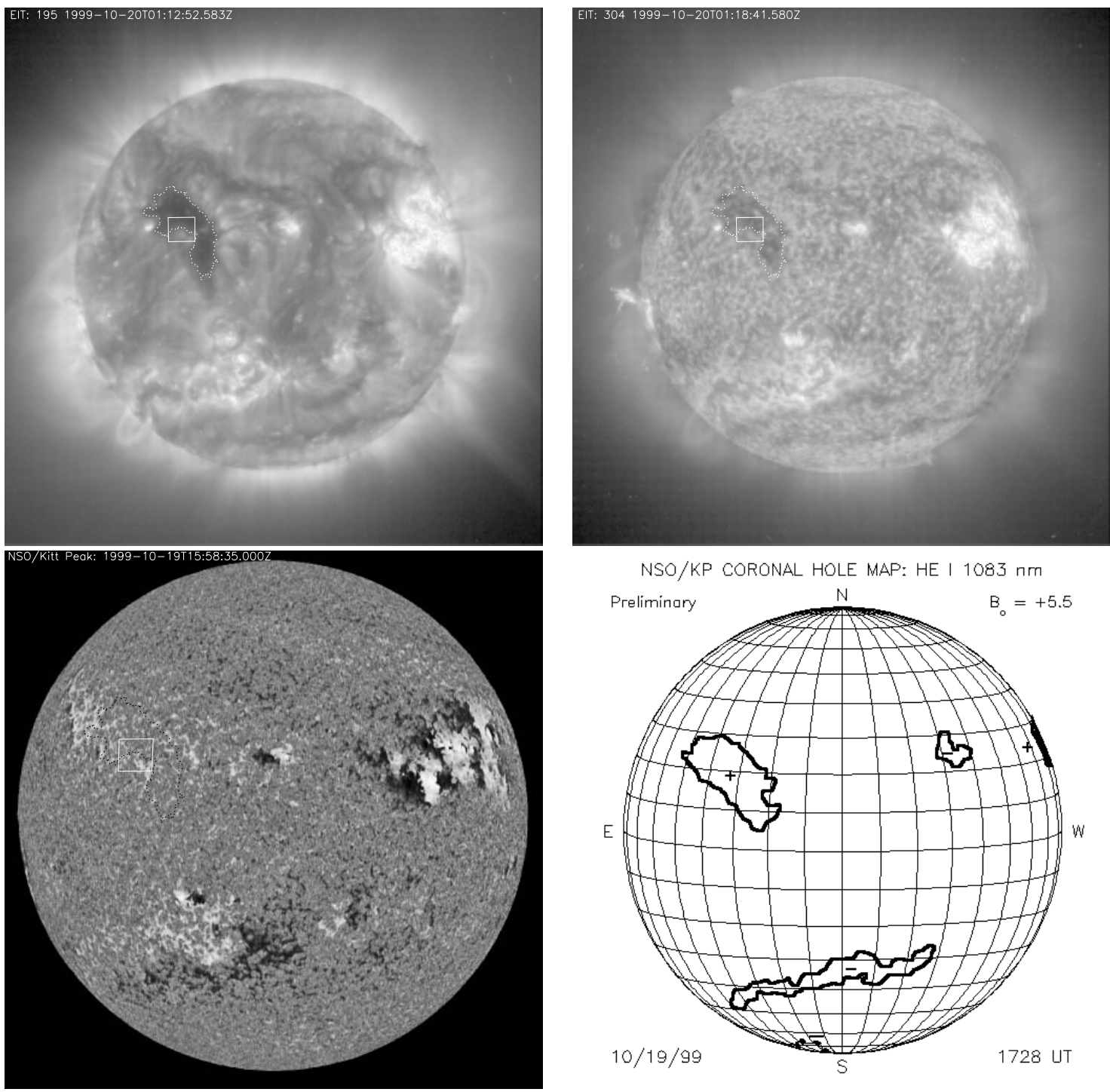

NSO/KP CORONAL HOLE MAP: HE I $1083 \mathrm{~nm}$

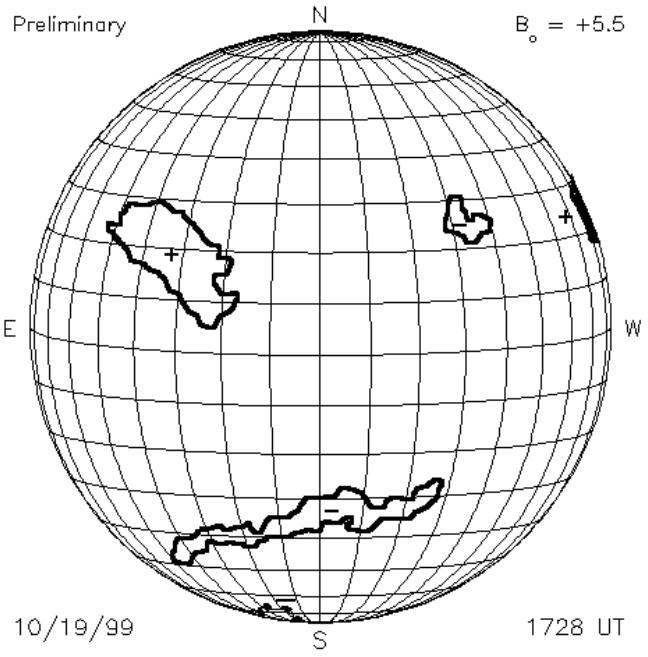

Figure 4.2: Context of the SUMER observation of a coronal hole on the disk on 19 October,

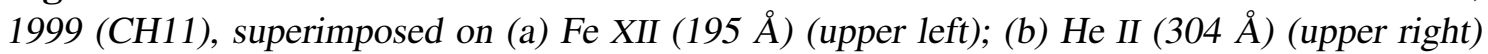
images taken by EIT at the same day; and (c) the magnetic field image obtained by NSO/KP at the same day. Note that the average magnetic field is about $6 \mathrm{G}$ in the coronal hole (bottom left). (d) The Kitt Peak coronal hole map for this ECH (bottom right).

map for this ECH is shown in bottom right panel. Another ECH observed on 19 October, 1999 (CH11) shown in Figure 4.2 gives similar results, but with an average magnetic field of $6 \mathrm{G}$ in the hole. It is essentially not same in the quiet Sun region. For a comparison, Figure 4.3 shows an example of the QS region observed at the disk center on 8 March, 1999 (QS1). The FOV of SUMER is superimposed on the EUV image taken by EIT at Fe XII (195 $\AA$ ) (left panel) and on the magnetogram taken by NSO/KP (right panel) on the same day. The magnetic field averaged across the FOV of SUMER is about $-0.3 \mathrm{G}$, which is close zero and indicates that this region has a nearly balanced flux of the magnetic field. 

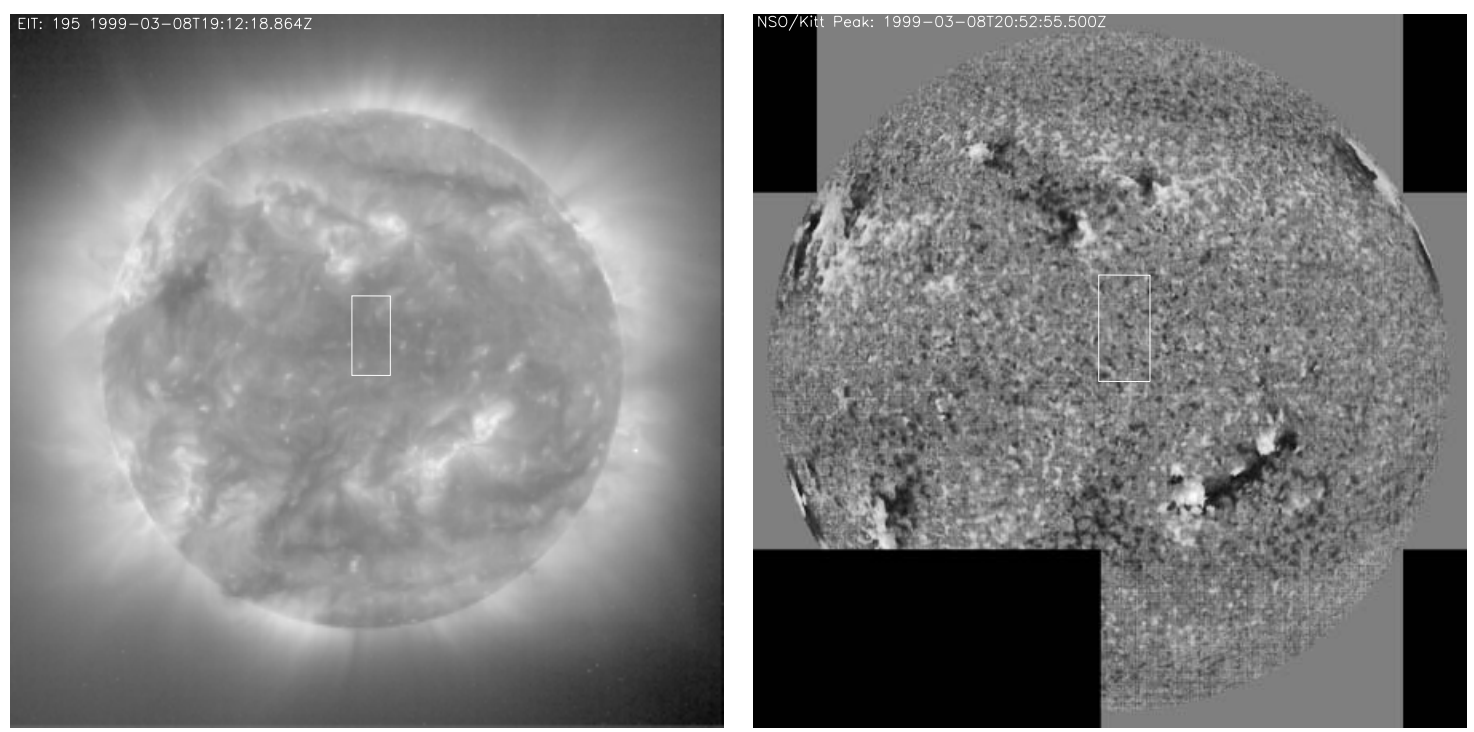

Figure 4.3: Context of the SUMER observation of a quiet Sun region at the disk center on 8 March, 1999 (QS1), superimposed on (a) Fe XII (195 A) taken by EIT (left), and (b) the magnetic field image obtained by NSO/KP (right) at the same day. Note that the average magnetic field is about $-0.3 \mathrm{G}$ in the field of view of the SUMER.

\subsection{Identification of lines}

As we have discussed in Section 3.2.2. SUMER can measure any FUV/EUV line in the wavelength range $500 \AA-805 \AA$ (2nd order) and $660 \AA-1610 \AA$ (1st order). The identification of FUV/EUV lines observed by SUMER has been done by some authors (Curdt et al., 1997, 2001; Feldman et al., 2000a) in various regions, such as the quiet Sun, coronal hole and above the limb. More than 1100 emission lines are available in the SUMER spectral range.

Unfortunately, the lines formed at high coronal temperatures are very small in number, owing to their low emission in coronal holes and blending with strong continua and cooler lines on the disk. The maximal formation temperature of the lines occurs for $\mathrm{Mg} \mathrm{X}$ $\left(1.1 \times 10^{6} \mathrm{~K}\right)$. This line is observed in 2 nd order of diffraction and has high amplification if detected on the bare portion of the SUMER detectors. Therefore, it can not be used in some holes, due to too low signal-to-noise ratio, if detected on the $\mathrm{KBr}$ portion of the detectors.

In Table 4.3 we list all FUV/EUV lines, formed at different temperatures between $1 \times 10^{4}$ $\mathrm{K}(\mathrm{C} \mathrm{I})$ and $1.1 \times 10^{6} \mathrm{~K}(\mathrm{Mg} \mathrm{X})$, which we are interested in and will analyse to deduce the maps of intensity, line-of-sight (LOS) velocity and line width. The rest wavelengths are taken from the literature listed in the table. The line formation temperatures are the same as those used by Teriaca et al. (1999), which were obtained using the data base of the Arcetri Spectral Code (Landini and Monsignori Fossi, 1990; Landi and landini, 2002). 
Table 4.3: Emission lines selected for this study

\begin{tabular}{c|c|c|c|c}
\hline Ion & Wavelength & Order & $\log _{10} T$ & Reference \\
\hline C I & & 1 & 4.11 & $\mathrm{~K}$ \\
Fe II & 1550.274 & 1 & 4.23 & $\mathrm{~K}$ \\
Si II & 1533.432 & 1 & 4.26 & $\mathrm{~K}$ \\
H I & & 1 & 4.30 & $\mathrm{~K}$ \\
O I & & 1 & 4.31 & $\mathrm{~K}$ \\
He I & 584.34 & 2 & 4.51 & $\mathrm{~K}$ \\
C II & $1036.3367 / 1037.0182$ & 1 & 4.71 & $\mathrm{KE}$ \\
Si III & $1296.726 / 1301.174$ & 1 & 4.78 & $\mathrm{~K}$ \\
Si IV & $1393.755 / 1402.770$ & 1 & 4.84 & $\mathrm{~K}$ \\
C IV & $1548.204 / 1550.781$ & 1 & 5.01 & $\mathrm{GK}$ \\
N IV & 765.148 & 2 & 5.15 & $\mathrm{~K}$ \\
O IV & $1401.156 / 787.711$ & $1 / 2$ & 5.23 & $\mathrm{~K}$ \\
N V & $1238.800 / 1242.778$ & 1 & 5.25 & $\mathrm{E}$ \\
S V & 786.480 & 2 & 5.26 & $\mathrm{~K}$ \\
S VI & 933.380 & 1 & 5.31 & $\mathrm{KM} 2$ \\
O V & 629.730 & 2 & 5.35 & $\mathrm{~K}$ \\
O VI & $1031.9261 / 1037.6167$ & 1 & 5.47 & $\mathrm{KM} 1$ \\
Ne VIII & 770.428 & 1,2 & 5.80 & SUMER \\
Mg IX & $694.004 / 706.058$ & 1 & 6.00 & $\mathrm{~K}$ \\
Mg X & 624.968 & 2 & 6.04 & SUMER \\
\hline
\end{tabular}

References - E: Edlén (1934); GK: Griesmann and Kling (2000); K: Kelly (1987); KE: Kaufman and Edlén (1974); KM1: Kaufman and Martin (1989); KM2: Kaufman and Martin (1993); SUMER: Dammasch et al. (1999c), Dammasch et al. (1999b) and Peter and Judge (1999).

\subsection{Determination of line parameters: intensity, position and width}

An important step in the analysis of emission line spectra is to measure the intensity, position and width of a line from its observed profile. In general, we assume that the spectral lines can be fitted with Gaussian profiles. This assumption is suitable for optically thin spectral lines emitted in the upper solar atmosphere. If a line is severely blended by other lines, multi-Gaussian fitting can be used. The form of a multi-Gaussian fit can be represented as

$$
I(x)=a+b x+c x^{2}+\sum_{i} I_{0 i} \exp \left[-\frac{\left(x-x_{0 i}\right)^{2}}{2 \sigma_{i}^{2}}\right],
$$

where $a, b, c, I_{0 i}, x_{0 i}$ and $\sigma_{i}$ are the parameters to be fitted to the observed line profile. $I_{0 i}, x_{0 i}$ and $\sigma_{i}$ are peak intensity, line center position and line width of the $i$ th line, respectively. Here we consider the case where the fitted profile has a considerable background 
level, which is considered by addition of a polynomial of order $n$ (we taken $n=1$ or 2). The integrated intensity $I_{i}$ of a line can be derived from the fit parameters, according to the property of a Gaussian function, and can be written as

$$
I_{i}=\int_{\lambda} I(\lambda) \mathrm{d} \lambda=I_{0 i} \sigma_{i} \sqrt{2 \pi}
$$

Several fitting algorithms have been discussed in the literatures and applied in order to obtain the best possible scientific results from the spectra (e.g., Judge et al., 1997; Chae et al., 1998c, Peter and Judge, 1999; Dammasch et al., 1999c). Generally, if the line is not, or only weakly, blended, the standard algorithm, i.e., fitting to a single Gaussian profile, can be chosen.

The standard fitting algorithm for the spectral decomposition unfortunately proved to be unstable, when applied to data with a low signal-to-noise ratio or faced with line profiles blended with each other. We therefore use a robust Genetic Algorithm (GA) with global optimization method, which was described in detail by Charbonneau (1995). An application in analysing CDS and SUMER spectra has been discussed in detail by McIntosh et al. (1998). However, a superposition by two or more lines with about the same wavelengths may result in a great uncertainty of the fitted parameters.

In our analysis of the SUMER spectra, we first applied the standard procedures for correcting and calibrating the data. Two methods were then applied to get the central position of a given line. First, we fitted the average profile to a single Gaussian or multi-Gaussians (hereafter "method 1"), thereby using the PIKAIA software (Charbonneau, 1995). Second, in order to deduce the Dopplergrams we calculated the central position for every pixel, by integrating the line intensity across a certain spectral window and determining subsequently the location of the 50\% level with sub-pixel accuracy (hereafter "method 2 "). This procedure was frequently used to obtain SUMER Dopplergrams (see for the details Dammasch et al., 1999c) and dramatically reduced the computing time for a large number of data. However, a systematic deviation of deduced central positions could occur if the selected spectral window included other lines (blends). This deviation can be corrected by comparing the average position deduced by this method with that obtained by the multi-Gaussian fitting.

A comparison of these two methods is studied, in which intensities, central positions and FWHM-widths of the C I line (1542.177 $\AA$ ) are determined by both methods. The data were observed in $\mathrm{CH}$. In Figure 4.4, the upper-left panel shows the average profile of this line, and other panels show its integrated intensities $(I)$, central positions $(p)$ and FWHMwidths $(w)$ varying with the spatial direction of the SUMER slit, where the dotted line represents results deduced by method 1 (denoted by 1 ) and the solid line deduced by method 2 (denoted by 2). The statistical results are listed in Table 4.4, in which the units of line parameters are the same as in Figure 4.4, and the quantity $\sigma$ denotes standard deviation. It should be mentioned that the $\mathrm{C}$ I line selected here is a weak one. If we compare the results deduced from a strong line, the difference should be even smaller. We therefore conclude that the results deduced by two methods are statistically consistent with each other. 

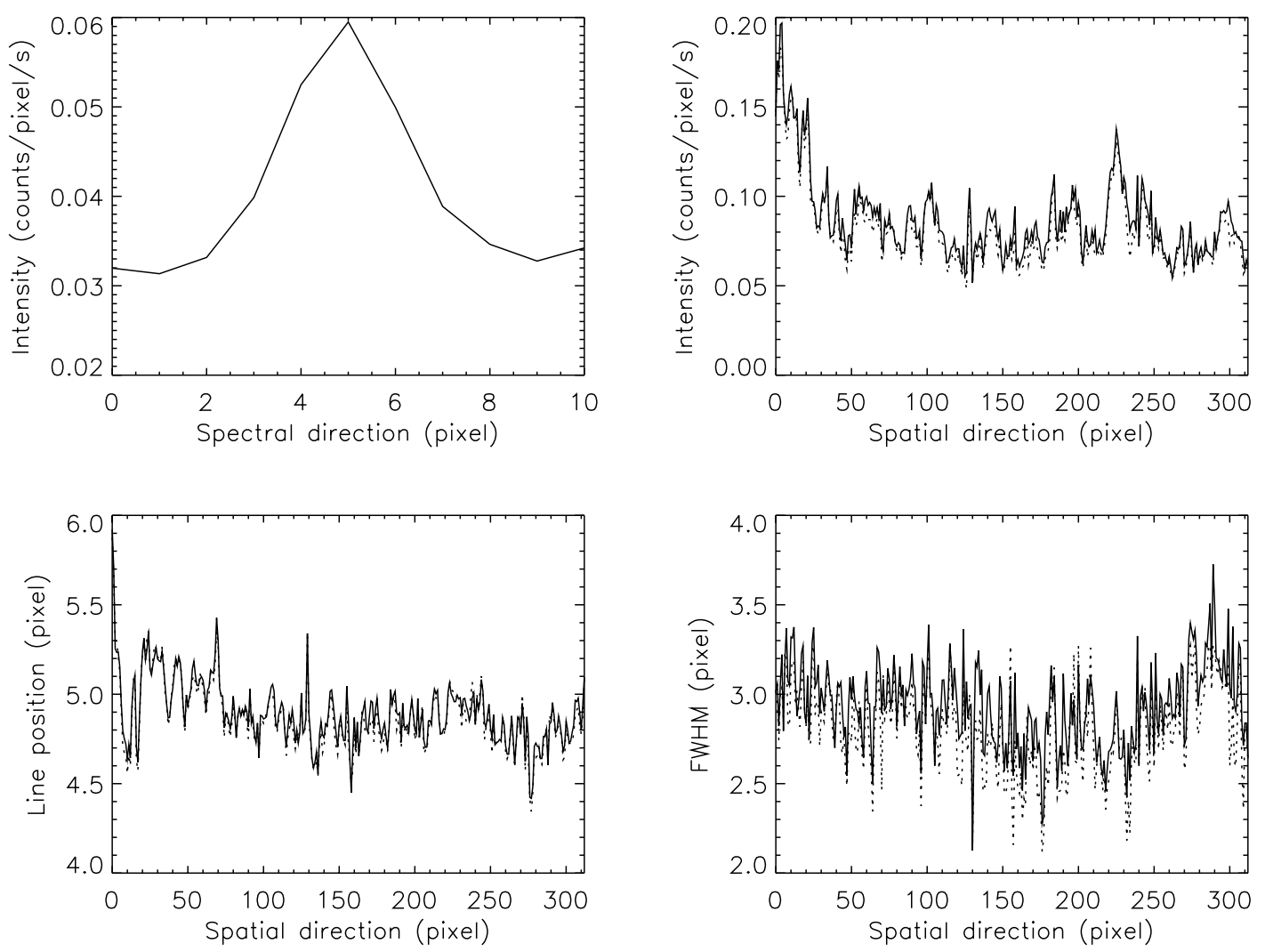

Figure 4.4: Comparison of two methods for deducing the line parameters. Top-left panel: average profile of the $C I$ line. Top-right panel: Intensity along the spatial pixels; Bottom-left panel: central position along the spatial pixels; Bottom-right panel: line width (FWHM) along the spatial pixels. Note that the dotted line represents the result deduced by method 1 and the solid line deduced by method 2.

Table 4.4: Comparison of two methods for deducing the line parameters

\begin{tabular}{c|c|c|c|c|c|c|c}
\hline $\bar{I}_{2}-\bar{I}_{1}$ & $\frac{\left|\bar{I}_{2}-\bar{I}_{1}\right|}{\bar{I}_{1}}$ & $\sigma_{\left(I_{2}-I_{1}\right)}$ & $\bar{p}_{2}-\bar{p}_{1}$ & $\sigma_{\left(p_{2}-p_{1}\right)}$ & $\bar{w}_{2}-\bar{w}_{1}$ & $\frac{\left|\bar{w}_{2}-\bar{w}_{1}\right|}{\bar{w}_{1}}$ & $\sigma_{\left(w_{2}-w_{1}\right)}$ \\
\hline 0.005 & $6 \%$ & 0.004 & 0.02 & 0.04 & 0.13 & $4 \%$ & 0.12 \\
\hline
\end{tabular}

\subsubsection{Discussion of estimating errors}

In order to evaluate the fitting errors for each line parameter, a so called "bootstrap" method has been applied. The application of this method to estimate the measurement uncertainties in astronomy was first discussed by Barrow et al. (1984). The method begins with a given data set obtained from the observation, and generates a series of pseudo data sets with a same number of the element as that of the original data set. The pseudo data samples will contain duplicates of some original data points, while some of original data points will thus not be contained. The same procedure as being used to fit the original data set will repeatedly be applied for all new data sets, and attain a set of resulting fit 

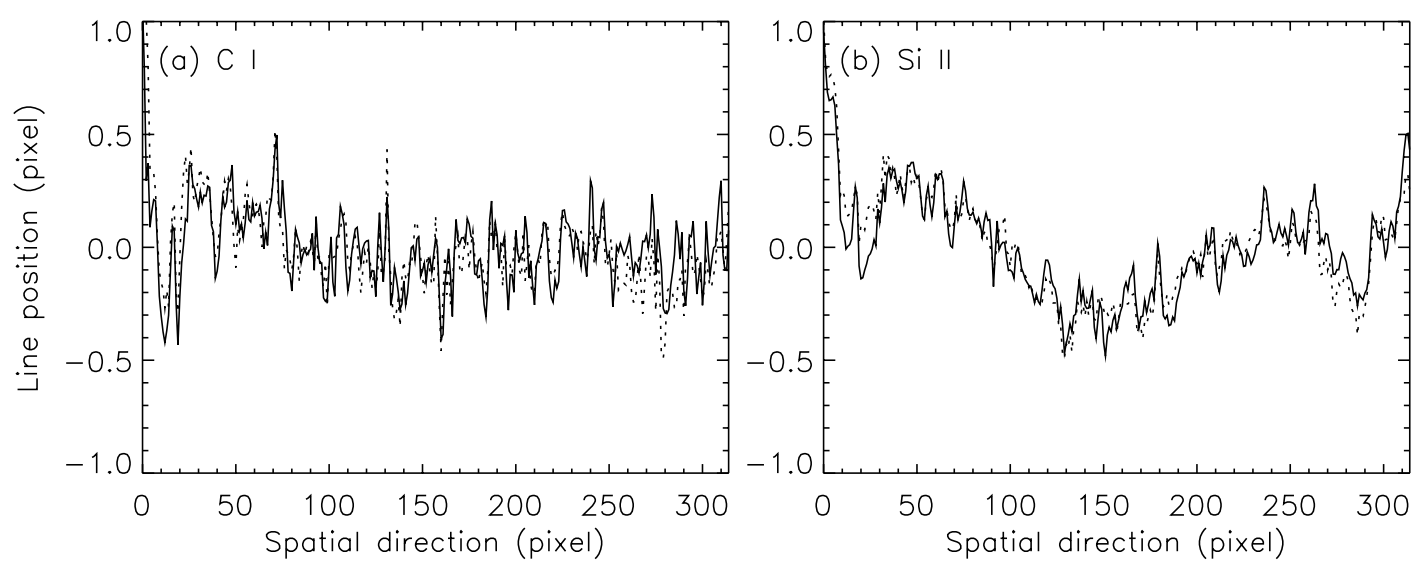

Figure 4.5: Residual errors of the line position after the geometrical correction using the standard software. (a) and (b) showing the central positions (relative to the mean position of each line, and averaged over 96 exposures) of the C I and Si II lines versus the spatial pixels. Note the solid line represents the result deduced from the $\mathrm{QS}$ data, while the dotted line from the $\mathrm{CH}$ data.

parameters, from which we can thus obtain the mean value and the standard deviation of each line parameter.

The errors given for Doppler shifts are calculated by the relation, $\sigma=\sqrt{\sigma_{f t}^{2}+\sigma_{r f}^{2}+\sigma_{g c}^{2}}$, where $\sigma_{f t}, \sigma_{r f}$ and $\sigma_{g c}$ denote the fitting error (as discussed above), the error of dispersion relation derived from the reference lines and the error of geometric calibration, respectively. We adopt a value of $1.5 \mathrm{~km} \mathrm{~s}^{-1}$ as the error resulting from the geometric calibration. The resulting total errors are found to be around $2 \mathrm{~km} \mathrm{~s}^{-1}$ for the most lines, as many authors have also estimated (see, e.g., Brekke et al., 1997; Chae et al., 1998c; Peter and Judge, 1999; Dammasch et al., 1999c).

\subsection{Additional geometrical correction}

The standard procedure for the correction of the geometrical distortion of the image and inclination of lines has been discussed in Chapter 4. Although this procedure can provide a good first-order correction to the geometric distortions, residual errors of line central positions are still present. According to the SUMER archive, the standard deviation of the residual errors was estimated to be 0.1 pixels (equivalent to about $0.8 \mathrm{~km} \mathrm{~s}^{-1}$ at $1540 \AA$ ) for spatial pixels 20 to 340, with a maximum deviation from the mean of about 0.25 pixels. As pointed out by Peter and Judge (1999), however, such residual errors are dependent of the pixel address on the detector, and may also change with the time. In addition, a spurious spectral line-shifts may also caused by thermal deformations of the instrument, when a time duration of an observation is long enough (see, e.g., Dammasch et al., 1999c). In order to deduce the Dopplergram more reliably, an additional line-position correction was performed, to remove spurious spectral line shifts caused by thermal deformations of the instrument, and to eliminate residual errors (systematically varying along the slit), 
remaining after the geometric correction using the standard software. The procedure is described in the following.

Before applying the procedure of additional line-position corrections, we show in Figure 4.5 the central positions (relative to the mean position of each line, and averaged over 96 exposures for each pixel address) of the C I and Si II lines varying with the spatial pixels. The data used here were obtained in a $\mathrm{CH}$ region $(\mathrm{CH} 6)$ and a QS region (QS1), respectively. SUMER was operated under the same conditions during the observations. From Figure 4.5, one finds that two data sets attained a rather similar tendency of the variation with the spatial pixels. The standard deviation of the residual errors from the mean is around 0.2 pixels. Large deviations can be found on the both brim parts of the slit. Because they were obtained in different regions, the similar tendency of the variation indicates that a physical effect caused by the Sun could be excluded (the physical effect of the Sun has been eliminated because the line profiles were obtained by averaging 96 exposures for each pixel address). Similar curves as shown in Figure 4.5 can be deduced for all lines, and used to make an additional geometric correction by subtracting them from the deduced Dopplergram for each line. However, such a procedure should be used very carefully, if different large structures are contained in the same FOV of the SUMER. Because large structures, such as active regions, $\mathrm{CH}$ regions and QS regions, may have a very different large-scale velocity field, the real Doppler shifts may not cancel even averaging over many exposures. In this case, a more reliable way is to use the QS data to correct for the $\mathrm{CH}$ data, but the precondition is that they are observed under the same instrumental condition, for example, in $\mathrm{CH} 6$ and QS1.

A similar method described in the paper of Dammasch et al. (1999c) can be used to correct for the systematic line-shifts caused by thermal deformations of the instrument. This spurious line-shifts are a function of the time, which can be deduced from the line positions averaged over the whole slit, and should be same for all lines in the same spectral window. We can thus use a strong cold line to deduce a standard curve (line position versus the time) and then applied to all lines in the same window.

\subsection{Wavelength calibration}

Wavelength calibration is a key step to determine the precise Doppler shift of a spectral line. In brief, we need to know how much a measured line deviates from its rest wavelength. After the shifted amount of the wavelength, $\Delta \lambda$, has been measured, the Doppler shift represented by velocity can thus be calculated by using the standard equation

$$
v=c \frac{\Delta \lambda}{\lambda_{0}}
$$

where $c$ is the speed of light, and $\lambda_{0}$ is rest wavelength. Usually, three methods have been applied for determining the quantity $\Delta \lambda=\lambda-\lambda_{0}$. 


\section{Lamp on board}

The most reliable method is using an onboard calibration lamp (e.g., Pt lamp), which has been applied in some rocket experiments (see, e.g., Rottman et al., 1990; Hassler et al., 1991). Unfortunately, the SUMER spectrometer has no such additional equipment.

\section{Zero shifts at the limb}

This method is based upon the physical assumption that above the limb all nonradial velocities should on average cancel each other out, i.e., the average Doppler shift must be zero after the solar rotation is corrected for. Thus we can determine the real Doppler shifts by calculating the shifted amount relative to the limb, and thus we need not know the rest wavelength. Previous studies have proved that such a method was indeed valid (see, e.g., Doschek et al., 1976;, Peter and Judge, 1999).

\section{Cold lines as reference lines}

This method is very useful for observations on the solar disk. Previous studies have shown that chromospheric lines have on average very small systematic line shifts. For example, Hassler et al. (1991) found the absolute shift of the Si II line (1533 $\AA$ ) to be $0 \pm 1.2 \mathrm{~km}$ $\mathrm{s}^{-1}$, by comparing the solar lines with the lines of an on-board calibration lamp. For the C I line (Chae et al., 1998c) only a small average red shift of about $1.5 \mathrm{~km} \mathrm{~s}^{-1}$ has been estimated. These chromospheric lines have a relatively low formation temperatures, they are therefore called cold lines. Such lines can be used as reference lines to establish a wavelength reference for observations on the disk. In this coordinate, the wavelength of a line can be determined relative to the chromospheric lines. Then its shift can be calculated by comparison with its rest wavelength. The estimated accuracy should be less than about $2 \mathrm{~km} \mathrm{~s}^{-1}$, as many authors have discussed (see, e.g., Feldman et al., 1982; Brekke et al., 1997; Chae et al., 1998c; Peter and Judge, 1999; Dammasch et al., 1999c). Lines with known, accurate laboratory wavelengths, such as Si I, S I, C I and O I, can be used in our study.

To apply this method, we synthesize the profiles of cold lines such as Si I, C I and O $\mathrm{I}$ in a spectral window based on their laboratory wavelengths and theoretical intensities under solar condition (Kelly, 1987). Then we compare our solar observations with this synthetic spectrum, and deduce the spectral dispersion ( $\mathrm{m} \AA /$ pixel). Using this dispersion, the Doppler shift of a "hotter" line can be then deduced relative to its neighbouring cold lines. 



\section{Chapter 5}

\section{Morphology of the Equatorial Coronal Holes}

\subsection{Introduction}

Spectroscopic and morphological studies of the equatorial coronal holes (ECHs) by means of measuring ultraviolet emission have been made since the Skylab time (see, e.g., Munro and Withbroe, 1972; Huber et al., 1974; Reeves, 1976). During the SOHO mission, SUMER and CDS have been used to observe both polar holes and equatorial holes. The SOHO mission began near the last solar minimum. At that time, equatorial coronal holes were very rare features due to a lack of active regions. During the solar maximum in 1999 , isolated equatorial coronal holes were frequently present on the solar disk. Therefore, most of the observations listed in Table 4.1 were carried out during that year. Some of them have been analyzed to study the morphology and statistical properties of equatorial coronal holes (see, e.g., Lemaire et al., 1999; Wilhelm et al., 2002; Feldman et al., 2000b). The results obtained by these authors have been described in Chapter 2. However, most of the data sets observed by SUMER in ECHs have not yet been analyzed. The main purpose of this work is to study the morphology of equatorial coronal holes, by using these data together with those obtained by EIT, MDI and NSO/KP. SUMER has both high spatial and spectral resolutions and a wide wavelength coverage, therefore our attention will be focused on the small structures because of their importance for understanding the physical processes concerning coronal heating and solar wind acceleration.

In this chapter, a brief description of the data selection is given in the next section; In section 3, the general properties of ECHs as seen in spectroheliograms of various lines and continua formed at different temperatures are described. The properties of Doppler shifts observed in different lines are presented in section 4; The various structures seen in spectroheliograms, Dopplergrams and their related underlying photospheric magnetic fields are discussed in sections 5, 6 and 7; In sections 8 and 9, we describe in brief temporal variations and a large transient event; Finally, the summary and discussion are given in the Section 10.

\subsection{Data selection}

In this study, we selected coronal holes that are among the largest ones. The field of view (FOV) of SUMER during these observations usually covered a disk area larger than about $100 \times 100 \operatorname{arcsec}^{2}$. Actually, the images obtained by the SUMER telescope were scanned slit by slit along the X-direction (East-West) of the Sun (raster scan mode), therefore they are not normal "pictures" that are taken simultaneously. The raster step size determines 
the spatial resolution along the X-direction, while that along the slit in Y-direction (NorthSouth) is approximately $1^{\prime \prime}$ for SUMER. Most of the observations for this study had high spatial resolutions with a raster step size of $1.13^{\prime \prime}$ along the X-direction of the solar disk. By help of this high spatial resolution, the small structures in the coronal holes can be investigated in details. Some of the observations have a raster step of $3^{\prime \prime}$ and thus cover a large area on the solar disk ( $\mathrm{CH} 13)$, which can be used to study the large-scale velocity field in ECHs. It should be mentioned that SUMER rastered along either the East-West direction or West-East direction, which is in the sense of the Sun's rotation, and therefore the effective field of view in this direction becomes either smaller or larger.

The exposure times of these observations were between $100 \mathrm{~s}$ and $300 \mathrm{~s}$ for most rasters. We could thus get a high enough signal-to-noise ratio of the signatures for deriving intensities, Doppler shifts and line widths reliably. For some spectral windows containing strong emission lines, for example, $760 \AA-790 \AA$ and $1020 \AA-1040 \AA$, short exposure times between $20 \mathrm{~s}$ to $60 \mathrm{~s}$ were also applied. With such a short exposure time and a fixed slit position (without raster step), rapid variations can be investigated.

The selected spectral windows include a number of lines (see line list in Table 4.3), whose formation temperatures span from less than $1 \times 10^{4} \mathrm{~K}$ to $1.1 \times 10^{6} \mathrm{~K}$. They are mainly emitted in the upper chromosphere, transition region and at the coronal base, respectively. Among these lines, we pay special attention to some lines emitted by Li-like ions of $\mathrm{O}^{5+}$ and $\mathrm{Ne}^{7+}$. They have a formation temperature of $0.3 \mathrm{MK}$ and $0.63 \mathrm{MK}$, respectively, a range which contains plasma information about a layer between the transition region and

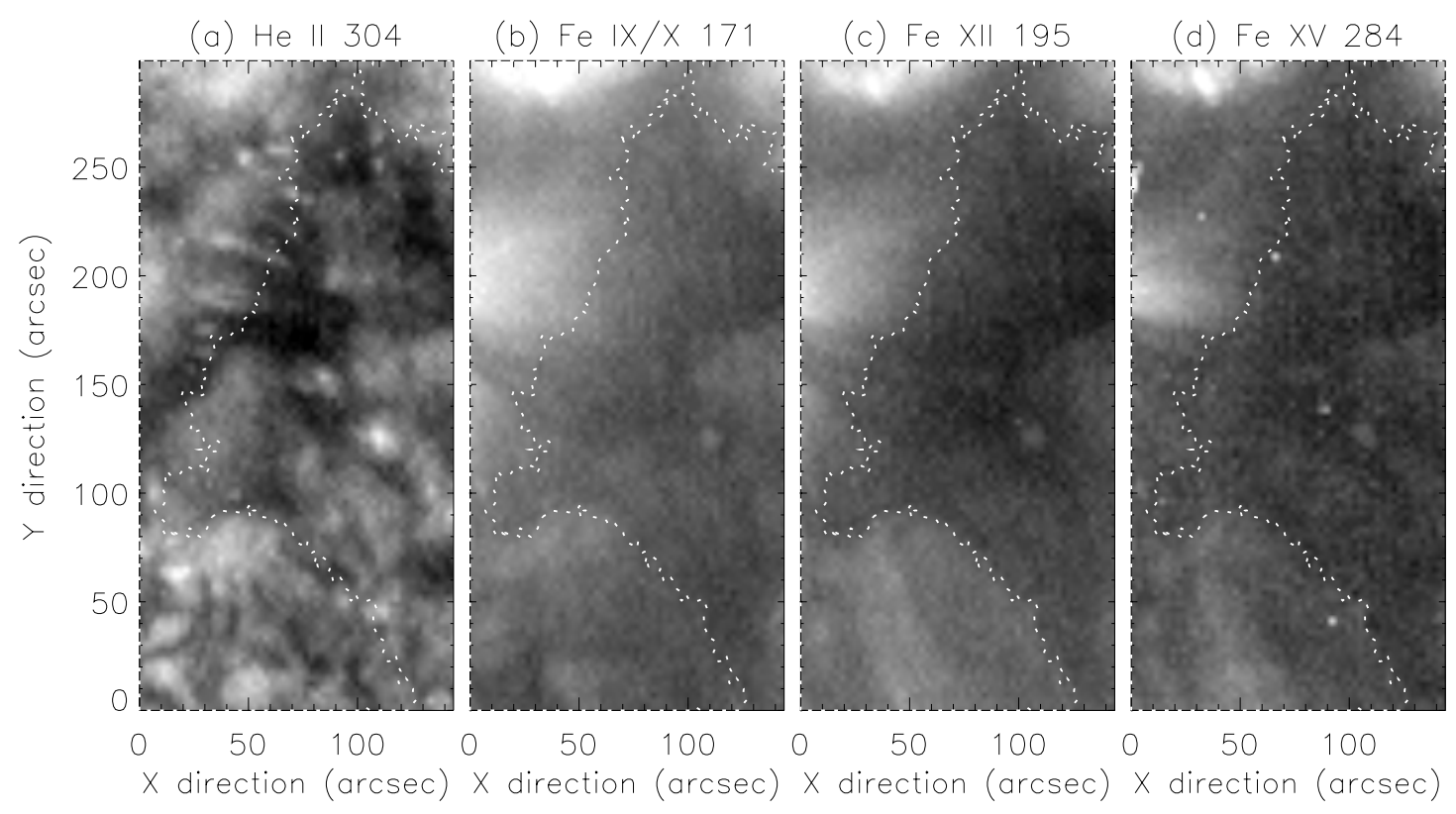

Figure 5.1: $\mathrm{CH} 6$, as seen in the EIT $304 \AA, 171 \AA, 195 \AA$ and $284 \AA$ channels, was observed on 11 March, 1999. Note that the regions inside and outside the coronal hole are separated by dotted lines, which are determined by the $\mathrm{CH}$ boundary as inferred from the image obtained in the Fe XII channel. 
corona. Previous studies have shown that, for example, a transition of averaged Doppler shifts from red to blue occurs at these temperatures. Another interesting line observed in the same spectral window as the $\mathrm{O}$ VI lines is the $\mathrm{H} \mathrm{I} \mathrm{L} \beta$. Like the other members of the Lyman series of hydrogen, this line is optically thick. Theoretical models suggest that the center part (core) of its line profile is formed at a temperature of about $20000 \mathrm{~K}$ in the upper chromosphere and low transition region, while the wings originate in the lower chromosphere (Vernazza et al., 1973; Gouttebroze et al., 1978). Therefore, its profile may contain information about the chromosphere over a wide temperature range.

An important objective of this study is to compare the morphology of coronal holes with that of the quiet Sun. The data observed in the quiet Sun during the days 8 and 9 March, 1999 were also selected (QS1). During these observations, SUMER was operated under the same conditions as when observing the coronal hole $(\mathrm{CH} 6)$ during the days 11 to 13 March 1999. In previous studies, people usually compared the coronal holes with their surrounding areas considered as quiet-Sun region. However, the formation of coronal holes is closely associated with active regions, so that their surrounding areas often contain the signatures originating from the active regions.

\subsection{Spectroheliograms}

Spectroheliograms (intensity ${ }^{1}$ maps) of various lines and continua ranging in wavelengths between $920 \AA$ and $1560 \AA$ are shown in Figures 5.2 to 5.7, as well as in Figures 5.11, 5.14, 5.20 and 5.21 in sections 5.5 and 5.7. Most data were obtained in $\mathrm{CH} 6$, which included 5 spectral windows and relatively complete line sets, although this coronal hole was not the largest one. In Figure 5.1, images obtained in the four channels of EIT in this hole are plotted. These images have the same FOV as the SUMER images. For a comparison, the corresponding spectroheliograms obtained in QS1 1 are plotted together with the ones obtained in $\mathrm{CH} 6$. The same scale of intensities is used to plot spectroheliograms for a given line. The regions inside and outside coronal holes are separated by dotted lines, which are determined by the $\mathrm{CH}$ boundaries as inferred from the images obtained in the Fe XII channel of EIT. The method has been described in Chapter 4. It should be noted that a logarithmic scale is used for all such maps to improve the image contrast.

\subsubsection{Chromospheric lines and continua}

The formation temperatures of chromospheric lines and continua shown here range from less than $1 \times 10^{4} \mathrm{~K}$ to $1.8 \times 10^{4} \mathrm{~K}$. Generally, the chromospheric network is well defined in all lines and the continuum emitted by this layer, and has similar properties in both inside and outside $\mathrm{CH}$, and QS regions.

\footnotetext{
${ }^{1}$ Although the word "intensity" is commonly used in astrophysical community, it should be mentioned that the use of "radiance" is standard in the International System of Units (SI).
} 
In the high-contrast images, the most prominent features seen in the $\mathrm{C} I$ line and the continuum around different wavelengths above $1100 \AA$ are small bright patches with enhanced emission in the network as well as in cell interiors (see Figures 5.2, 5.3, 5.4, 5.6 and 5.7, as well as in Figures 5.11 and 5.14 in section 4). These features have spatial sizes of about $2^{\prime \prime}-10^{\prime \prime}$, are larger and brighter in the network, and usually elongated in the slit direction. Similar results have been also found in QS regions by other authors (see, e.g., Landi et al., 2000; Feldman et al., 2001). The phenomenon that the patterns are longer in the slit direction has been interpreted as being caused by the fact that the lifetimes of these bright points are shorter than the total integration time. For instance, if a bright point has a size of $5^{\prime \prime}$ in diameter, the total time for the slit scanning across this feature is about 10 min, thereby assuming that the exposure time is $2.5 \mathrm{~min}$ and the raster step size is $1.13^{\prime \prime}$, so that we can only get a size of about $2^{\prime \prime}$ along the raster direction (solar X-direction) if the lifetime of the feature is less than $5 \mathrm{~min}$.

These features are not clear in cell interiors when seen in the continuum with wavelengths between $930 \AA$ and $1100 \AA$ (see Figures 5.5.5.20 and Figure 5.21). One possible reason is that emissions at these wavelengths are weak. In the cases the lower photon flux from cell interiors may reduce the signal-noise ratio of the signatures and thus lead to disappearance of such small structures.

For the Fe II, Si II and O I lines, which have formation temperatures between $1.5 \times 10^{4} \mathrm{~K}$ and $1.8 \times 10^{4} \mathrm{~K}$, the bright points discussed above become less pronounced (see Figures 5.5, 5.6, and 5.7, as well as in Figures 5.21, 5.11 and 5.14).

However, the appearance of the chromospheric features seen in lines of hydrogen and helium is somehow different from those found in other lines and continua. In Figures 5.4 , 5.5 and 5.21, spectroheliograms of the He I (584 $\AA)$, H I L $\beta$ (1025 $\AA$ ) and H I L-11 (918 $)$ lines show that the chromospheric network is wider and more diffuse. Loop-like structures are often found like in transition region lines that we will discuss in the following. Similar result has also been found in the continuum at $710 \AA$ (Wilhelm et al., 2002).

\subsubsection{Transition region lines $\left(T_{e}<0.5 \mathrm{MK}\right)$}

All transition region ions selected for this study have very strong emission lines, which thus can be used to deduce line parameters reliably. The formation temperatures of these lines range from $5 \times 10^{4} \mathrm{~K}$ to $3 \times 10^{5} \mathrm{~K}$. Some of them are emitted by Li-like ions such as $\mathrm{O}^{5+}, \mathrm{N}^{4+}$ and $\mathrm{C}^{3+}$, which have extended contribution functions versus the temperature.

In general, the chromospheric network is well defined in all transition region lines. The locations of enhanced emission in these lines coincide with those in chromospheric lines, but appear more extended into the cell interiors if seen in high-contrast images. The network width, as seen in all transition region lines, tends to be broader in the $\mathrm{CH}$ than in the QS. Loop-like structures are the most prominent features in the transition region for both the $\mathrm{CH}$ and QS regions. They have visible widths of about $10^{\prime \prime}$ and lengths of about $10^{\prime \prime}-30^{\prime \prime}$, and can be seen more clearly in lines emitted by Li-like ions, but also in the $\mathrm{H}$ 
I, He I and C III lines. The lifetime of some loops can be estimated to be longer than 30 min for a loop with a length of $20^{\prime \prime}$. From the intensity images, a difference between the $\mathrm{CH}$ and QS regions is not obvious, except for the He I line, which appears to be weaker in the hole.

Using data with the high spatial resolution provided by SUMER, such loop-like structures have been found to be the most striking features in the quiet Sun when seen in transition region lines (see, e.g., Warren and Winebarger, 2000, Wilhelm et al., 2002, Landi et al., 2000; Feldman et al. 2000b, 2001). Feldman et al. (2001) found that most of such loops straddle the chromospheric network and have no visible connections with the bright points seen in chromospheric lines. Co-aligning the MDI magnetogram with the SUMER image, Warren and Winebarger (2000) found that most of such structures had no connection with the intranetwork magnetic field with mixed polarities as found by Dowdy (1993). They suggested that these fine features may be related to spicules with highly dynamic properties.

In our case for coronal holes, this topic will be further discussed in Section 5.7, by comparing the O VI spectroheliograms and Dopplergrams with the chromospheric network and MDI magnetograms.

\subsubsection{Upper transition region and coronal lines $\left(T_{e}>0.5 \mathbf{M K}\right)$}

The two lines, which are emitted by Li-like ions of $\mathrm{Ne}^{7+}$ at $770 \AA$ and $\mathrm{Mg}^{9+}$ at $625 \AA$, are obtained in the 2nd order (see Figures 5.2 and 5.3). On the disk, emissions in these two lines stem mainly from the upper transition region and coronal base. The majority of loop-like structures, which can be seen in transition region lines, are invisible in the $\mathrm{CH}$ region except for some large ones. In the QS region, the chromospheric network is still distinguishable in the Ne VIII line, but appears more diffuse than in other lines. In the $\mathrm{CH}$ region, the network is in this line almost absent and only small remnants still exist. A further study of this line will be presented in section 5.5, in which a more complicated morphology will be discussed.

The $\mathrm{Mg}$ X coronal line is very dark and uniform in coronal hole, while in the QS region it is more complicated. Here the network disappears, but some bight structures can still be seen. Actually, the $\mathrm{Mg} \mathrm{X}$ line is the only coronal line that we can use to deduce images of the coronal hole from our data. In addition, a similar property can be found in the $\mathrm{Mg}$ IX line obtained in ECH (Wilhelm et al., 2002). 

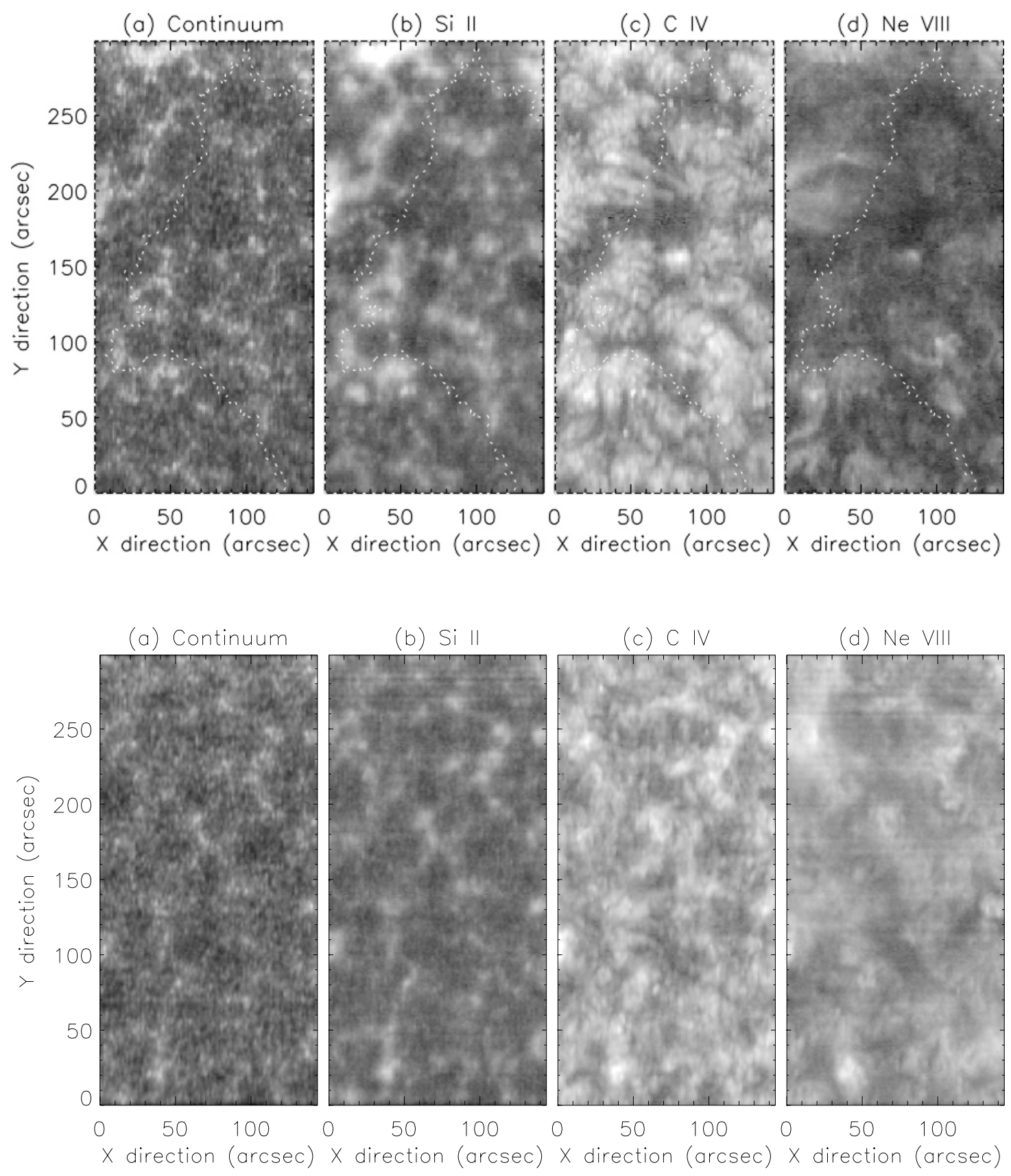

Figure 5.2: Intensity maps (logarithmic scale) obtained in the spectral window 1532 A to 1552 $\AA$ : (a) continuum (around $1539 \AA$ ); (b) Si II (1533 A); (c) C IV (1548 $)$ ); and (d) Ne VIII (770 in 2nd order). Upper panel: CH6 observed on 11 March, 1999; Bottom panel: Q\$1 observed on 8 March, 1999 at the center of the Sun. 

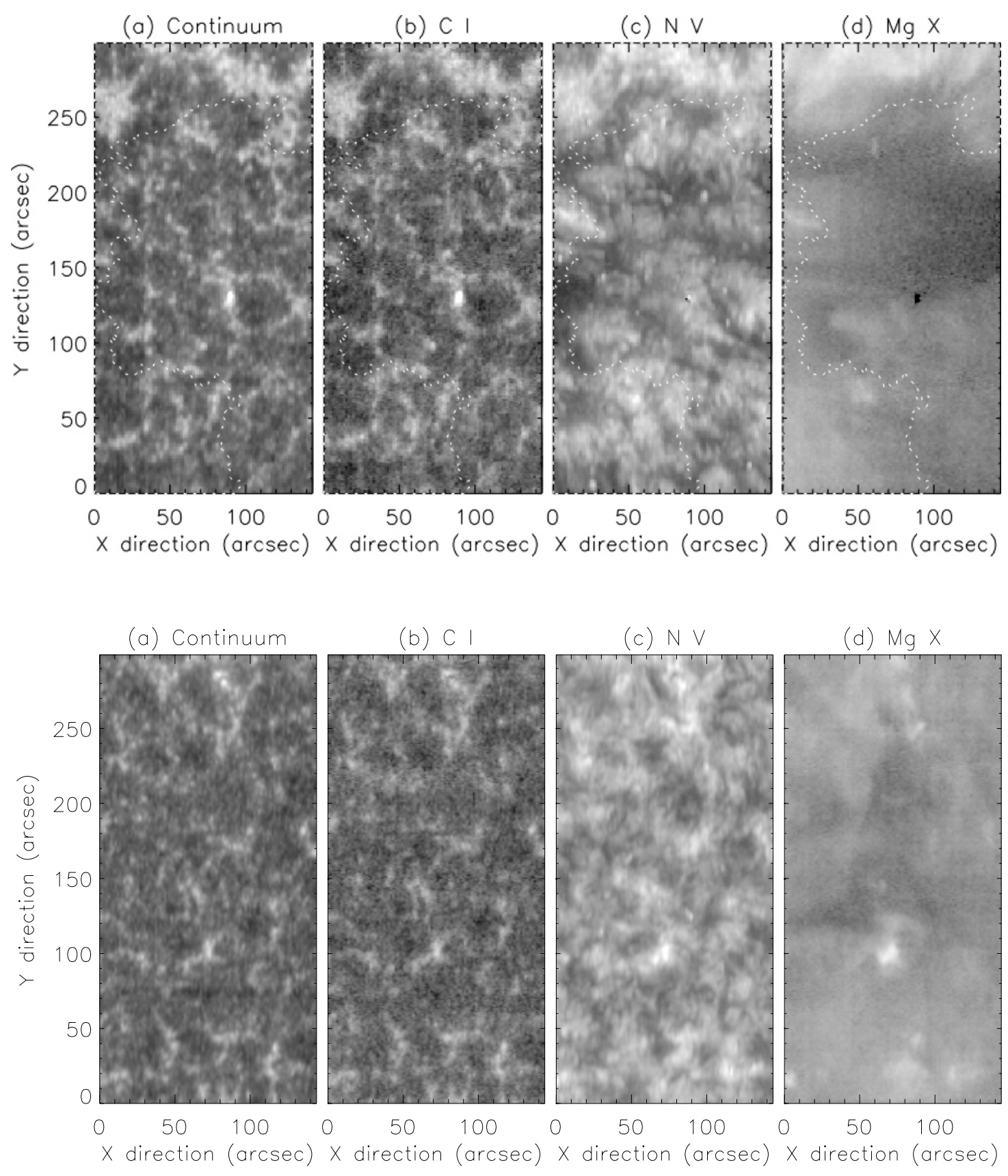

Figure 5.3: Intensity maps (logarithmic scale) obtained in the spectral window $1233 \AA$ to 1253

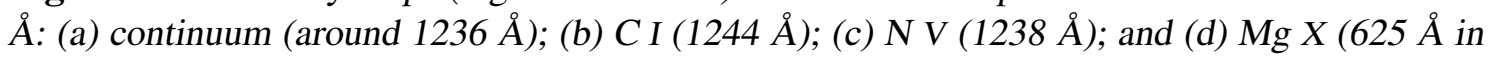
2nd order). Upper panel: CH6 observed on 12 March, 1999; Bottom panel: QS1 observed on 8 March, 1999 at the center of the Sun. 

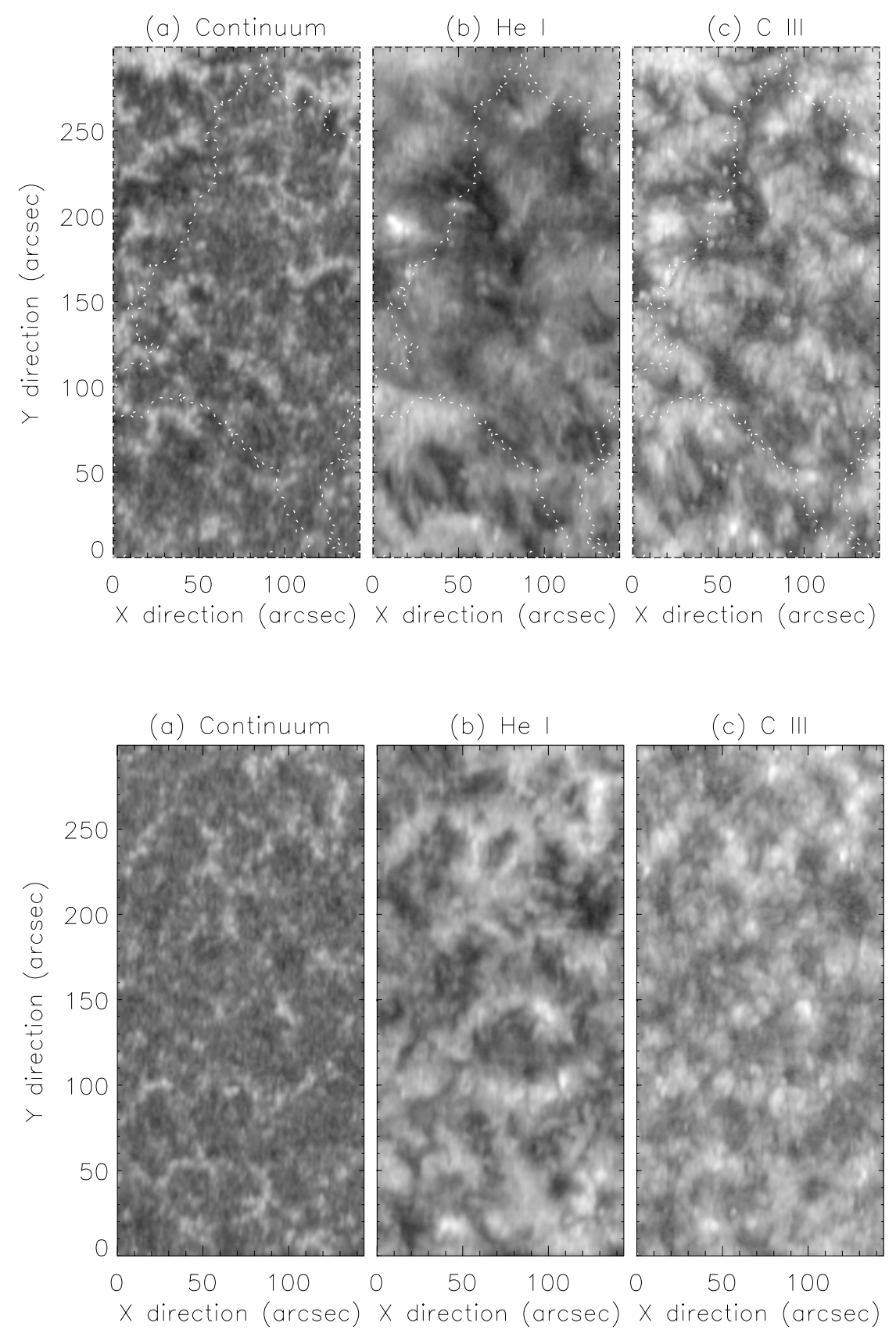

Figure 5.4: Intensity images (logarithmic scale) obtained in the spectral window $1160 \AA$ to 1180 $\AA$ : (a) continuum (around $1170 \AA$ ); (b) He I (584 $\AA$ in 2nd order); and (c) C III (1175 $)$ ). Upper panel: $\mathrm{CH}$ [ observed on 8 March, 1999; Bottom panel: QS1 observed on 8 March, 1999 at the center of the Sun. 

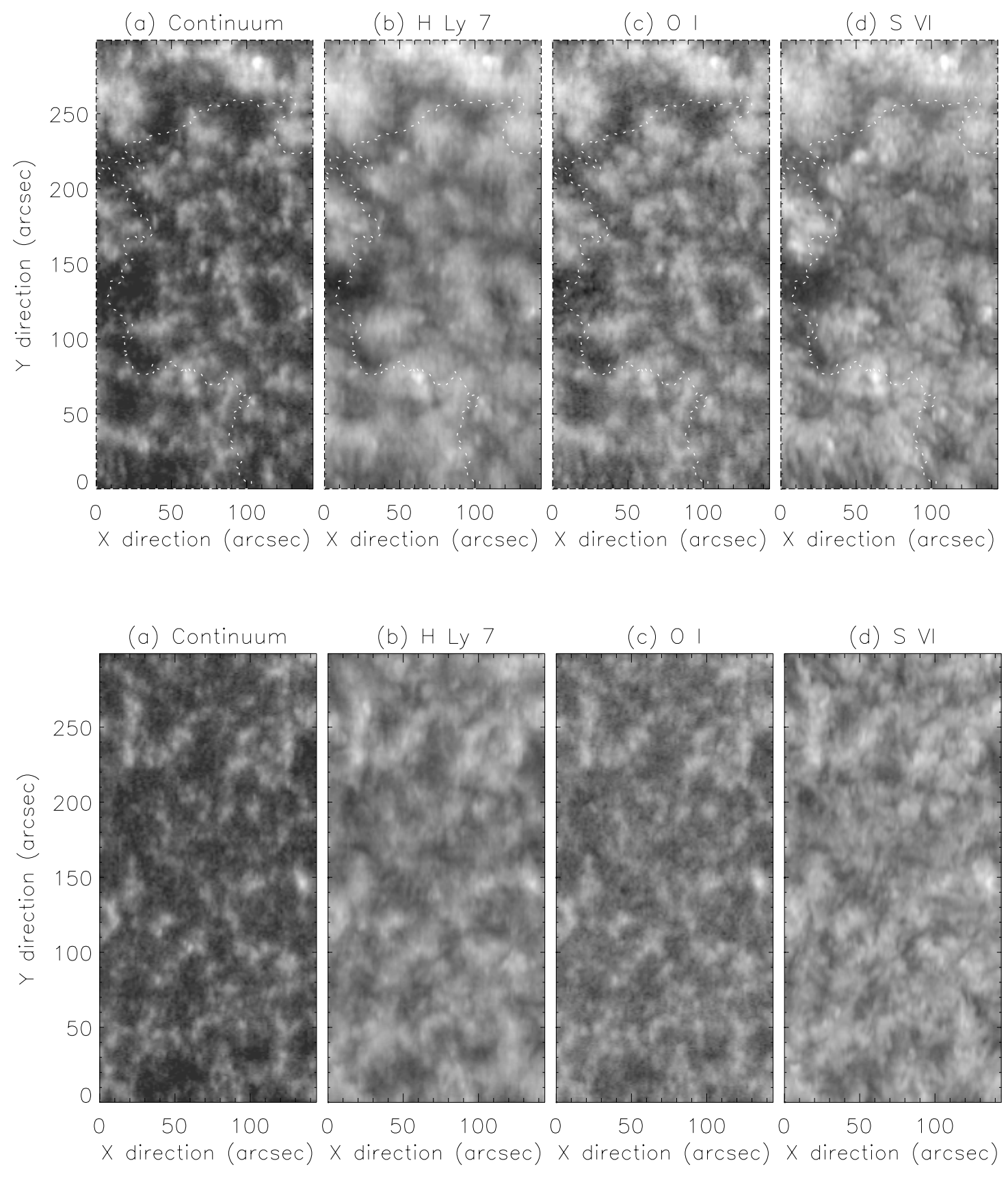

Figure 5.5: Intensity maps (logarithmic scale) obtained in the spectral window $915 \AA$ to $935 \AA$ : (a) continuum (around $932 \AA$ ); (b) H I L-7 (926 A); (c) O I (929 $)$ ); and (d) S VI (933 Å). Upper panel: CH6 observed on 13 March, 1999; Bottom panel: Q\$1 observed on 8 March, 1999 at the center of the Sun. 


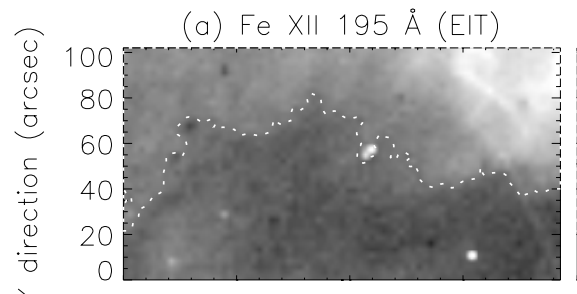

(d) N IV

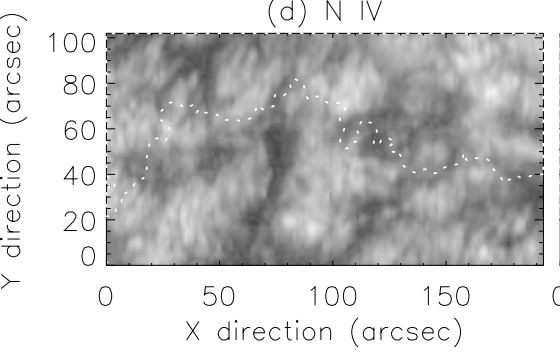

(b) Continuum

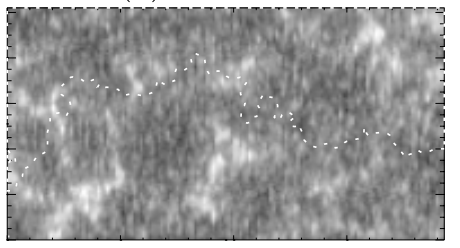

(e) C IV

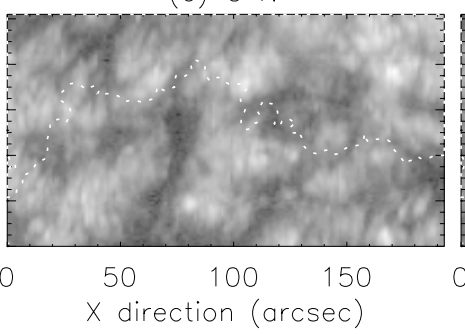

(c) $\mathrm{Fe} \mathrm{II}$

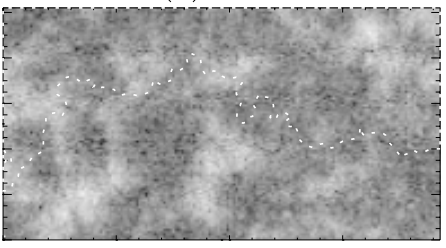

(f) $\mathrm{Ne} \mathrm{VIII}$

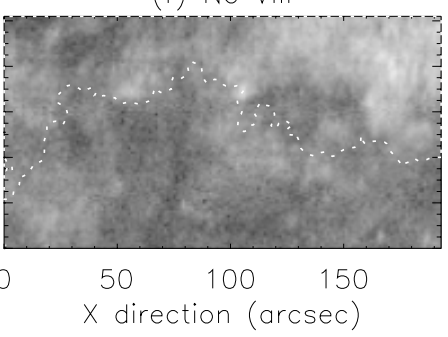

Figure 5.6: Intensity maps (logarithmic scale) obtained in the spectral window $1525 \AA$ to 1565 $\AA$ : (a) CH11 seen in the EIT $195 \AA$ channel; (b) continuum (around $1540 \AA$ ); (c) Fe II (1563 $\AA$ ); (d) NIV (765 $\AA$ in 2nd order); (e) C IV (1548 $)$ ); and Ne VIII (770 $\AA$ in 2nd order). All lines were simultaneously obtained by SUMER in CH[11 on 23 October, 1999.

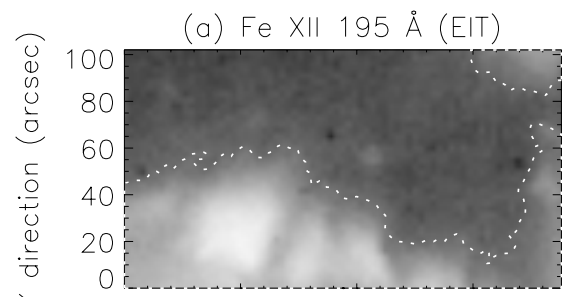

(d) N IV

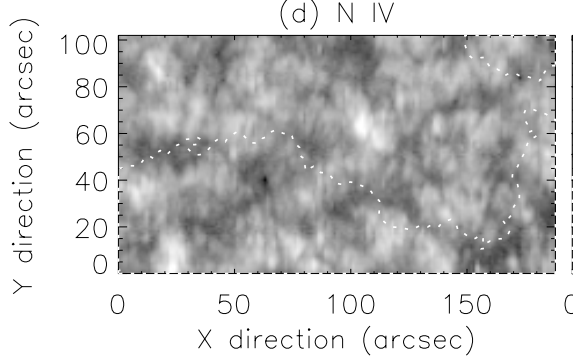

(b) Continuum (1522 $)$

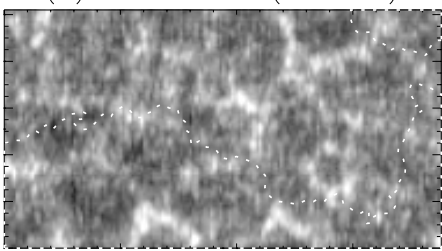

(e) $\bigcirc \mathrm{V}$

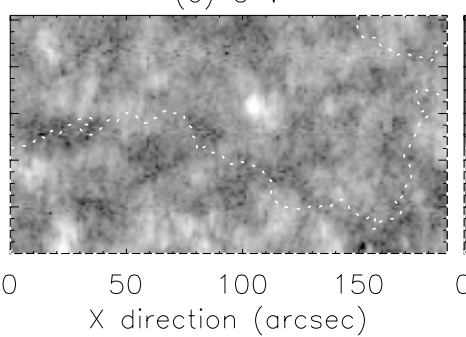

(c) Si 11

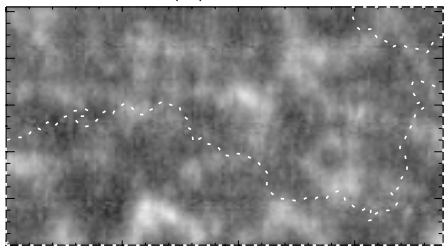

(f) $\mathrm{Ne} \mathrm{VIII}$

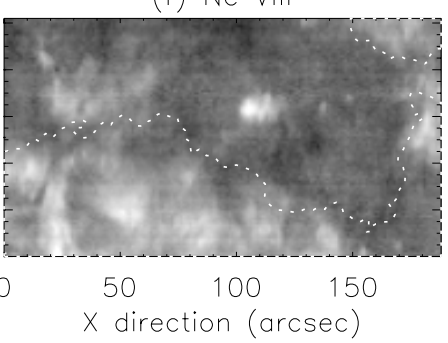

Figure 5.7: Intensity maps (logarithmic scale) obtained in the spectral window $1525 \AA$ to 1545 $\AA$ : (a) CH12 seen in the EIT $195 \AA$ channel; (b) continuum (around $1522 \AA$ ); (c) Si II (1533 $)$ ); (d) N IV (765 $\AA$ in 2nd order); (e) O V (760 $\AA$ in 2nd order); and Ne VIII (770 $\AA$ in 2nd order). All lines were simultaneously obtained by SUMER in CH[12 on 29 October, 1999. 


\subsection{Doppler shifts}

The velocity field in the line-of-sight is derived at different wavelengths shown in Figures 5.8 and 5.9. Four chromospheric lines are O I, Si II, H I L-11 and He I. The C IV and $\mathrm{N} v$ lines represent typical properties of transition region lines, while the $\mathrm{Ne}$ VIII and $\mathrm{Mg}$ $\mathrm{X}$ lines reveal the flow behaviour of plasma coming from the coronal base. We describe some general properties of Doppler shifts in coronal holes deduced from these lines. A more detailed discussion about this topic will be given in the following sections, where the data of magnetic fields are analyzed together with the SUMER data. The data used here were observed on 11, 12 and 13 March, 1999 in the same equatorial hole (CH6). The blue color represents the outflow velocities (blue shifts), while the red color displays the inflow (red shifts). The color bar above each figure indicates the full-velocity scale for each line. The boundaries of the hole region have been marked by dotted lines, and the hole areas can be identified as the dark region in Figure 5.1. For transition-region and coronal lines, an absolute wavelength calibration has been made related to chromospheric lines, which will be discussed in Chapter 6. For the chromospheric lines, we have simply set their average Doppler shift to $0 \mathrm{~km} \mathrm{~s}^{-1}$ (no absolute wavelength calibration) to deduce their Dopplergrams in lack of cooler lines relative to their formation temperature. However, it is generally believed that they have only small shifts on average.

\subsubsection{Chromospheric and transition region lines $\left(T_{e}<0.5 \mathbf{M K}\right)$}

By inspection of Figure 5.8, a first impression may be that the Dopplergram obtained in the $\mathrm{O}$ I and $\mathrm{Si}$ II lines is quite different from that deduced from the $\mathrm{H} \mathrm{I} \mathrm{L-11} \mathrm{and} \mathrm{He} \mathrm{I}$ lines. These lines are all formed between $15000 \mathrm{~K}$ and $20000 \mathrm{~K}$, but the latter two have a complicated formation mechanism. The difference in the blue and red shifts of the $\mathrm{H} \mathrm{I}$ and $\mathrm{He} \mathrm{I}$ lines is apparently larger than that of the $\mathrm{O}$ I and Si II lines. A similar result can also be found in other coronal holes. An even larger contrast of the Doppler shifts can be deduced from another member of Lyman series, H I L $\beta$, which we will discuss in the section 5.7, where we show how they are related to the opacity in the network.

Compared with the intensity maps of these lines, it appears that the Doppler shifts of the O I and Si II lines have a close relation with the network structure. The redder shifts are mainly from the intranetwork, while the bluer ones mainly from internetwork (cell interiors). It is interesting to find out whether such a relationship is associated with the underlying convective motions or caused by local magnetic activity in the cell interiors and "red shifts" of transition region lines in the network.

As was described in chapter 2, transition region lines of $\mathrm{C}$ IV and $\mathrm{N} V$ have been found to be generally redshifted except for some small-scale areas. This can be confirmed again in our Dopplergrams (see Figure 5.9). In fact, the average Doppler shift of the $\mathrm{C}$ IV line deduced from different observations is different. In our data obtained in $\mathrm{CH} 6$, the average red shift is $5.9 \mathrm{~km} \mathrm{~s}^{-1}$ and only about $6 \%$ are blue shifted. A value of $2 \mathrm{~km} \mathrm{~s}^{-1}$ has been deduced by Dere et al. (1989b), with a wavelength calibration determined by the 


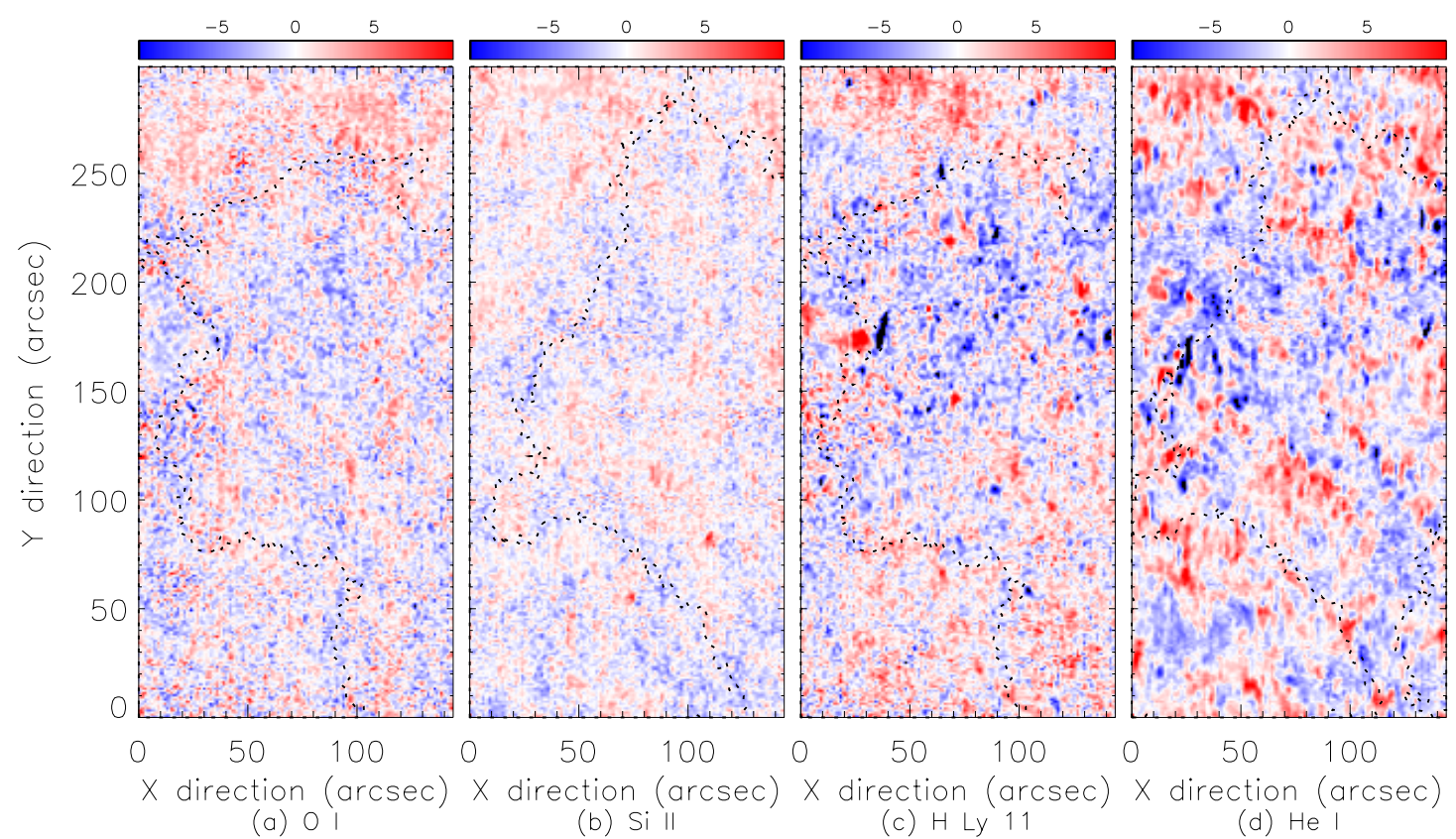

Figure 5.8: Dopplergrams of chromospheric lines for $\mathrm{CH} 6$ (full scale: $\pm 10 \mathrm{~km} \mathrm{~s}^{-1}$ ): (a) $\mathrm{O}$ I (929 $\AA)$; (b) Si II (1533 $\AA$ ); (c) H I L-11 (918 $)$ ); and (d) He I (584 $\AA$ in 2nd order). This CH was observed on 11, 12 and 13 March, 1999 (CH6).

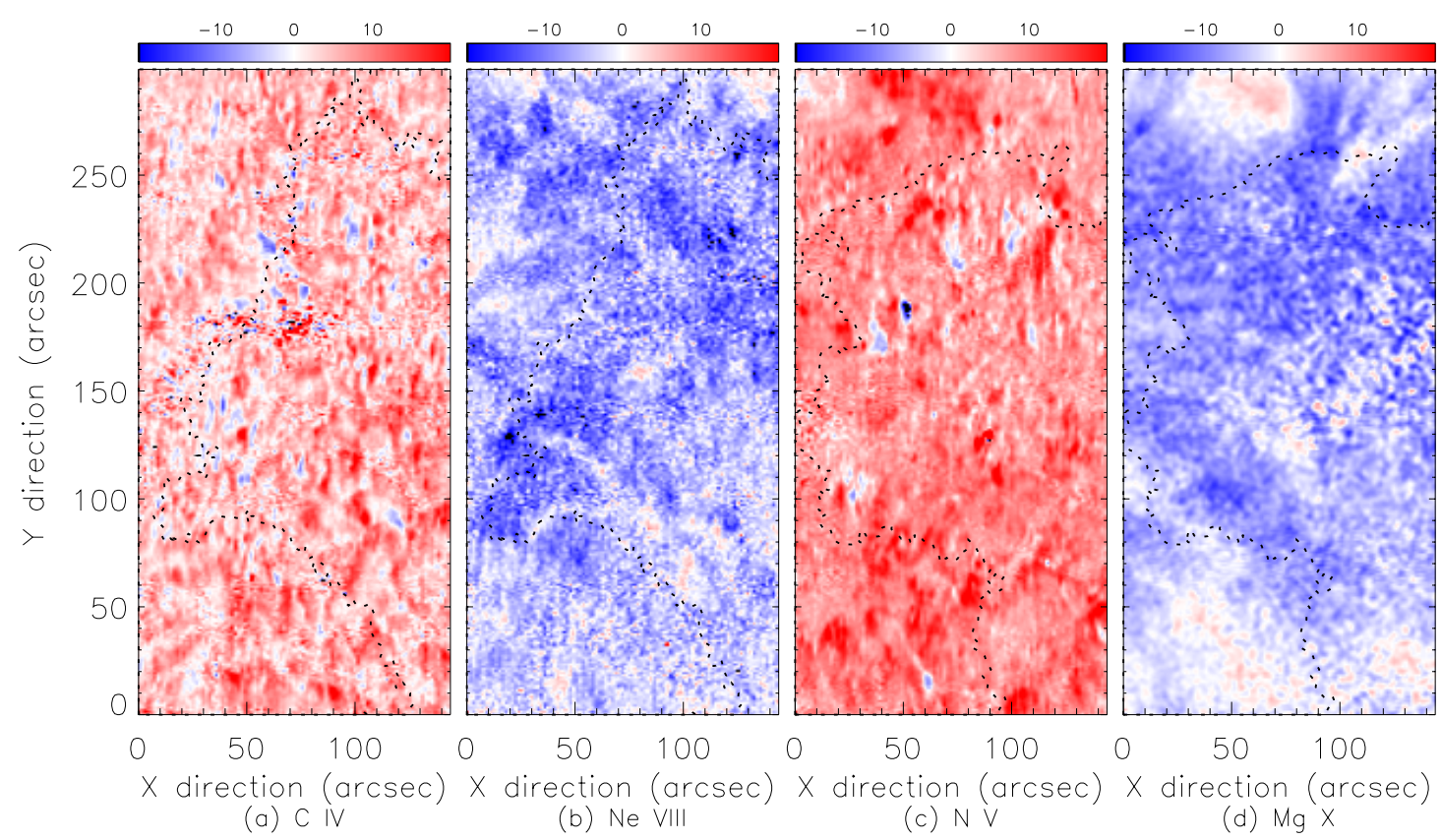

Figure 5.9: Dopplergrams of transition region and coronal lines for $C H 6$ (full scale: $\pm 20 \mathrm{~km}$ $\left.s^{-1}\right)$ : (a) C IV (1548 $\AA$ ); (b) Ne VIII (770 $\AA$ in 2nd order); (c) N V (1238 $)$ ); and (d) Mg X (625 in 2nd order). The $C H$ was observed on 11 and 12 March, 1999 (CH 6 ). 
chromospheric line Si I. They found that there is a proportion of $26 \%$ that are blue shifts, as compared with 7\% in the quiet Sun. On the other hand, Peter and Judge (1999) and Peter (1999) deduced an average red shift in a polar hole and found 67\% of the area is filled by blue shifts. It is possible that a measurement uncertainty may affect the ratio of blue to red shifts. On the other hand, such a difference may also result from the different magnetic geometry prevailing in the coronal hole, a presumption which will be discussed in the following sections.

\subsubsection{Upper transition region and coronal lines $\left(T_{e}>0.5 \mathrm{MK}\right)$}

From Figure 5.9, it can be seen that the Ne VIII and $\mathrm{Mg}$ X lines are on average blue shifted in the coronal hole, and have a very different behaviour in Doppler shifts if compared with other chromospheric and transition region lines. The red shifts are present only in some loop-like structures. Moreover, more structures can be seen in Ne VIII than in Mg X, like it is seen in their intensity maps. Except for some bright regions, the Dopplergram of the $\mathrm{Mg}$ $\mathrm{X}$ line is more homogeneous in the hole area. It seems that this result is in agreement with a geometry of the coronal funnel, namely, the corona must be more uniform magnetically at higher altitude. It is worth mentioning that large blueshifts may come also from regions outside the hole, indicating that open field lines also originate there. This should be not surprising because previous studies have demonstrated that the boundary of a coronal hole could be rather different when defined in different wavelengths (Kahler et al., 1983; Neugebauer et al., 1998; Bromage et al., 2000).

The velocity field deduced from the Ne VIII line will be discussed further in section 5.6, in which we find that it is obviously associated with the underlying chromospheric network.

\subsection{Network, bright structures and underlying magnetic fields}

During the days 3-8 November, 1999, the SUMER telescope was pointed to a large equatorial coronal hole and traced the darkest parts with raster scans. The selected spectral window was centered around $1540 \AA$, which includes lines of Si II (1533 $),$ C IV (1548 and $1550 \AA$ ) and Ne VIII (770 $\AA$ in 2 nd order). The formation temperatures of these lines span from about $1.8 \times 10^{4} \mathrm{~K}$ to $6.3 \times 10^{5} \mathrm{~K}$. The lines are emitted in the upper chromosphere, transition region and at the coronal base, respectively. The SUMER data selected for this study were taken on 3 and 5 November $(\mathrm{CH} / 3$ in Table 4.1). As defined in the Fe XII channel of the EIT image, the two data sets were indeed obtained from the same hole, but in different areas. This coronal hole is shown in Figure 5.10. Its boundary defined by the EIT Fe XII (195 A) channel is outlined by a white dotted line. The overlaid box-shaped window indicates the field of view (FOV) of SUMER. 
For this study, the photospheric magnetogram was obtained by anonymous ftp from the NSO (National Solar Observatory) at Kitt Peak. The used Fe I (8688 $)$ ) magnetogram was taken between 17:48 UTC and 18:43 UTC on November 5 and 23:09 UTC and 00:03 UTC on 3 and 4 November, with a spatial resolution element of $1.14^{\prime \prime} \times 1.14^{\prime \prime}$. Following the documentation on the field data, we have multiplied the instrumental values by a factor of 1.46, thus converting them to magnetic field strength given in Gauss. A detailed description of the measurement has been given in Jones et al. (1992). We finally co-aligned the magnetogram and the SUMER image in the Si II line by computing the cross correlation between the two images. This procedure is also applied to co-align the SUMER and EIT image.
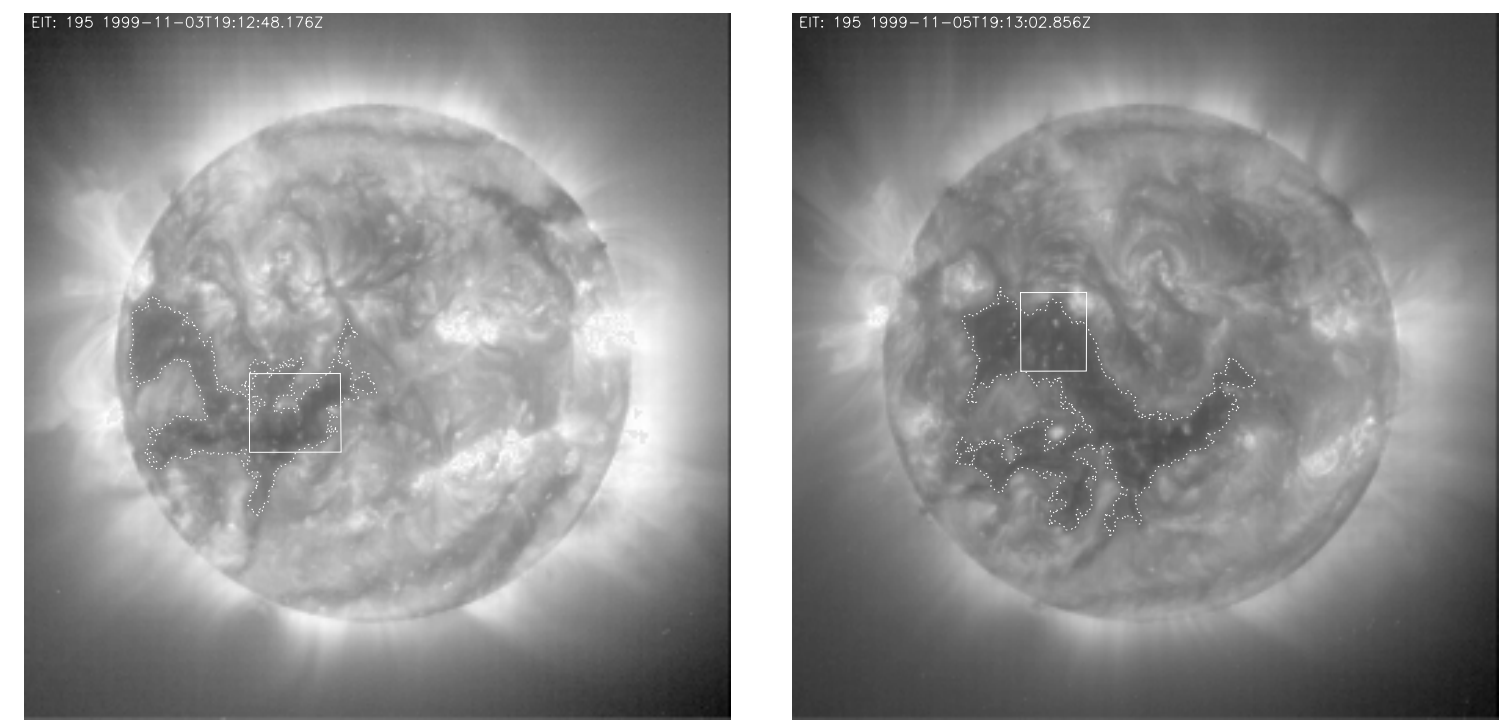

Figure 5.10: The equatorial coronal hole $(\mathrm{CH} / 13)$ as seen in the $195 \AA$ channel of EIT, with the field-of-view of SUMER being superimposed as a white box. Left panel: 3 November, 1999; Right panel: 5 November, 1999.

\section{Case 1: CH13 observed on 5 November, 1999}

The correspondence between the measured magnetogram and EUV images is shown in Figure 5.11, in which we plot together the spectral intensity maps obtained by SUMER and EIT in different lines and magnetic field contours (with levels of $-20 \mathrm{G},-10 \mathrm{G}, 10$ $\mathrm{G}$ and $20 \mathrm{G}$ ) measured by NSO/Kitt Peak. In Figure 5.11, the network structure (seen as a bright pattern in the Si II line and the continuum) corresponds to the concentrations of magnetic flux. The magnetic network structure in this coronal hole is very stable during the SUMER observation, and the image of the Si II and continuum brightness and the magnetic network are well coinciding spatially. The magnetic map indicates that this hole is occupied predominantly by white patches indicating positive polarity of the field. The average net magnetic flux density (signed) is about $7 \mathrm{G}$ inside the hole, with an uncertainty 

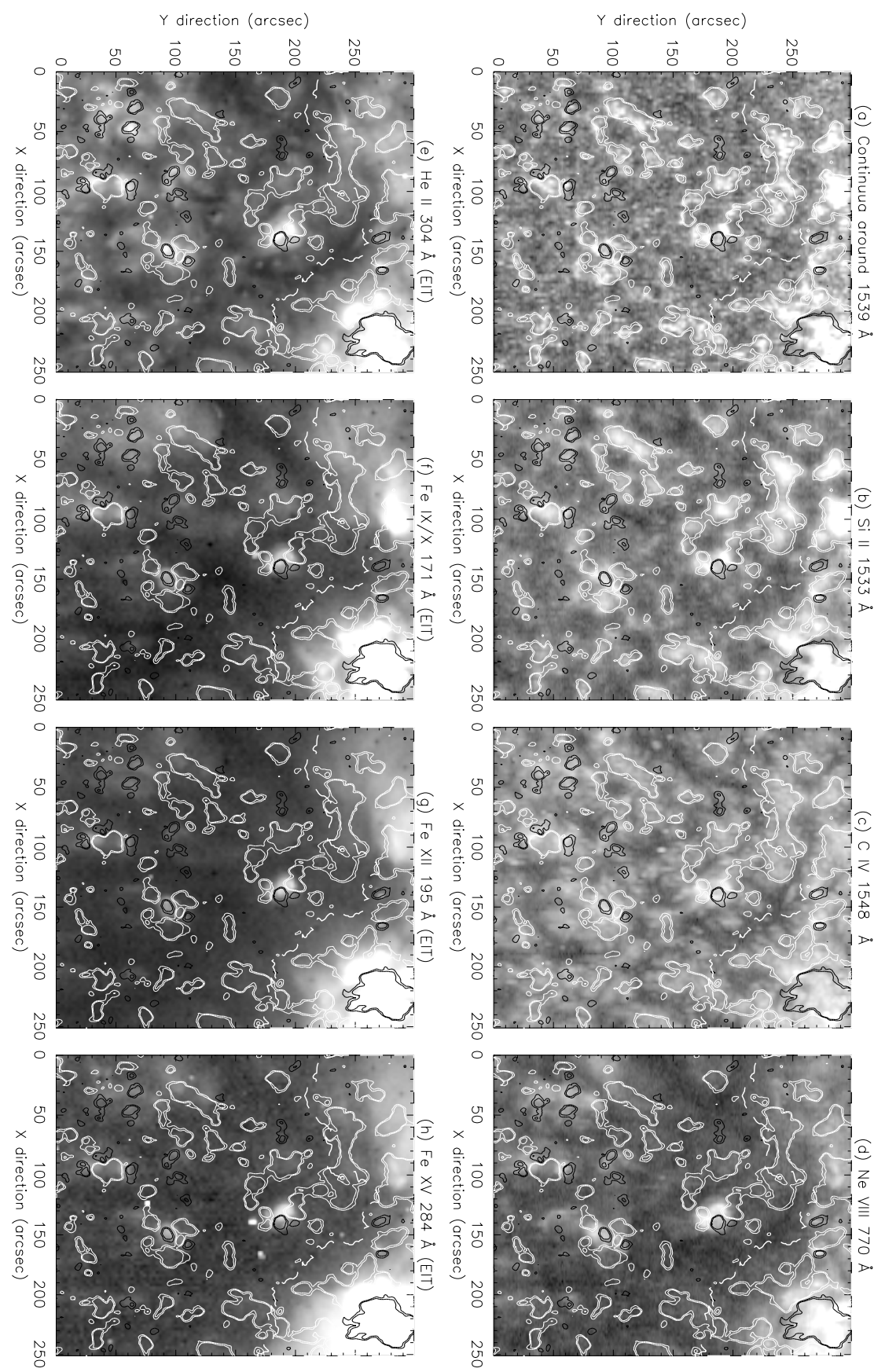

Figure 5.11: Network and bright points seen in different lines observed SUMER, EIT and NSO/KP on 5 November, 1999. Left panel: $C H 13$ seen in the EIT $304 \AA, 171 \AA, 195 \AA$ and $284 \AA$ channels. Right panel: Image scanned by SUMER covering a part of this hole as seen in the continuum, Si II (1533 $\AA), C$ IV (1548 $\AA$ ) and Ne VIII (770 $\AA$ in 2nd order) lines, respectively. Contour plots in both panels represent magnetograms of the same area observed by NSO/Kitt Peak, with the white lines representing the positive polarity of the line-of-sight field component and the black line the negative polarity (contour levels: $-20 \mathrm{G},-10 \mathrm{G}, 10 \mathrm{G}$ and $20 \mathrm{G}$ ). The chromospheric network is indicated by the intensity of the cooler line of Si II and by the magnetic field. Both data sets are consistent with each other. 


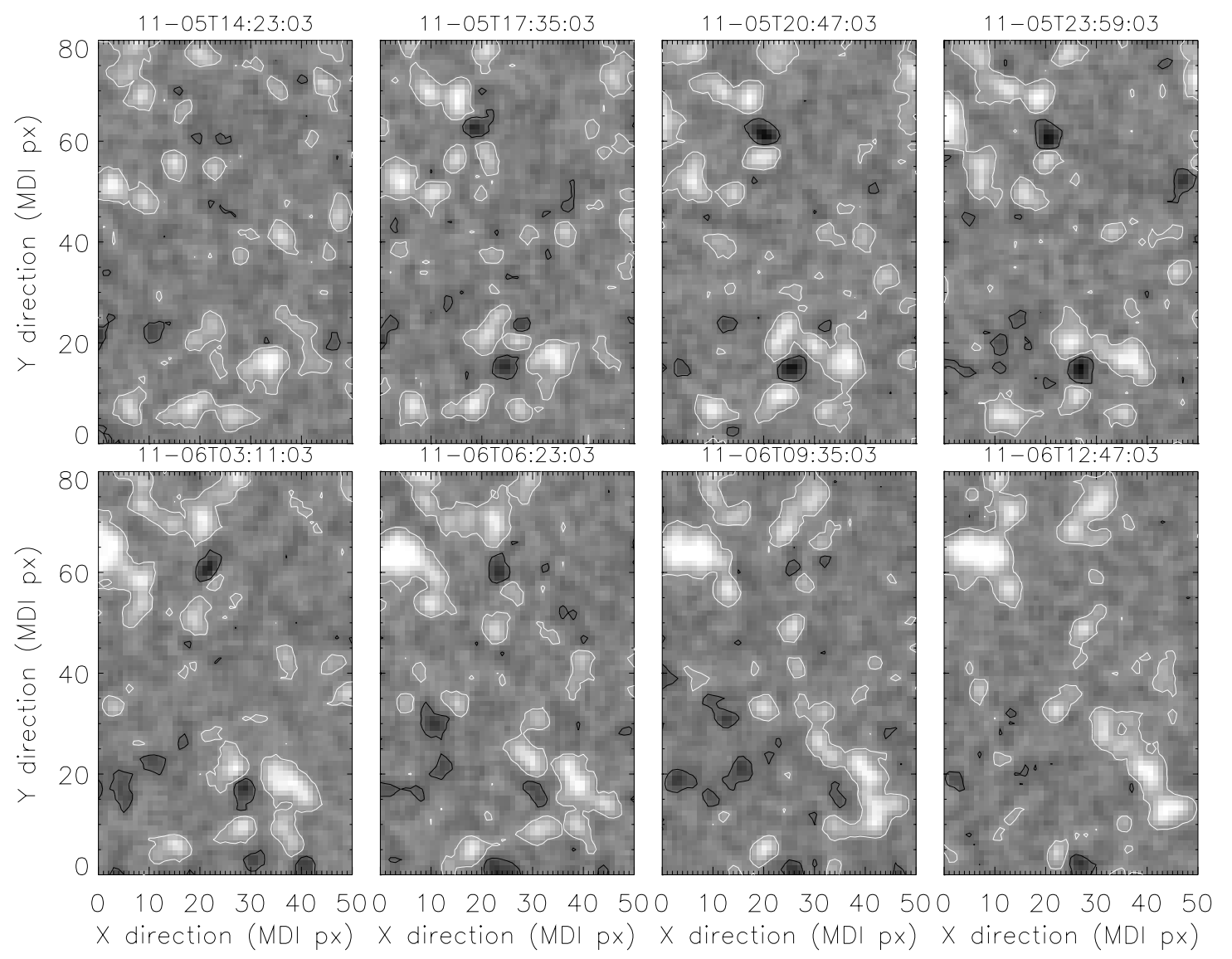

Figure 5.12: The evolution of the bright points seen in magnetograms observed by MDI. Contour levels: $-10 \mathrm{G}$ (black lines) and $10 \mathrm{G}$ (white lines). The frames are by about 3 hours separated in time.

of less than $1 \mathrm{G}$ (J. Harvey, 2002, private communication). There were also some mixedpolarity magnetic features in this $\mathrm{CH}$ (see also the left panel of Figure 5.15). The negative flux occupied about $25 \%$ of the total unsigned flux in the $\mathrm{CH}$ area.

From Figure 5.11, it can be found that the coronal hole is well defined as dark area in the Ne VIII line observed by SUMER and coronal emission observed by EIT, where the network structure is almost invisible inside the $\mathrm{CH}$. However, the hole is not uniformly dark even seen in the intensity map obtained at the highest temperature of about $2 \mathrm{MK}$, which is the formation temperature of the Fe XV line (284 $\AA$ ). The isolated bright structures inside the hole can be identified as bright points (BPs), as discussed in Section 2.1.5. Such bright points are associated spatially with regions where mixed-polarity magnetic features are present (Figure 5.11). Moreover, if seen in the cooler lines (Si II and C IV) and continuum, bright points appear as part of the network and are not easily distinguished from the normal part of the network due to the low contrast between their brightnesses. The intensity ratio between the bright point and the average normal network is estimated to be 0.9 for Si II and 1.3 for C IV. Therefore, by means of the Si II and continuum brightness alone, we are not able to distinguish the polarity of the field. Both polarities are present, 
but they cannot be discriminated, since both types of fields appear to be associated with similar, bright plasma features when seen in this chromospheric line. The relative area occupied by such bright points is estimated to be about $14 \%$. The intensity ratio between bright points and dark regions is on average about 3.3 for the Ne VIII line.

With the 96-minutes magnetic field data obtained by MDI, we can trace the evolution of the bright points inside this hole. In Figure 5.12, two of them, which can be found in the FOV of SUMER at $\mathrm{X}=140^{\prime \prime}, \mathrm{Y}=190^{\prime \prime}$ and $\mathrm{X}=150^{\prime \prime}, \mathrm{Y}=100^{\prime \prime}$, respectively, are plotted with a time interval of about $3 \mathrm{~h} 12 \mathrm{~m}$ from 14:23 UTC on 5 November to 12:47 UTC on 6 November. In the FOV of MDI, they are located (in pixel coordinates) at $X=20, Y=60$ and $\mathrm{X}=30, \mathrm{Y}=15$ ( $1 \mathrm{MDI}$ pixel equals about $2^{\prime \prime}$ ), respectively. Figure 5.12 clearly shows the formation and decay process of these two BPs. It can be inferred that their lifetimes are both about 20 hours, falling in the typical range for bright points. On the other hand, the
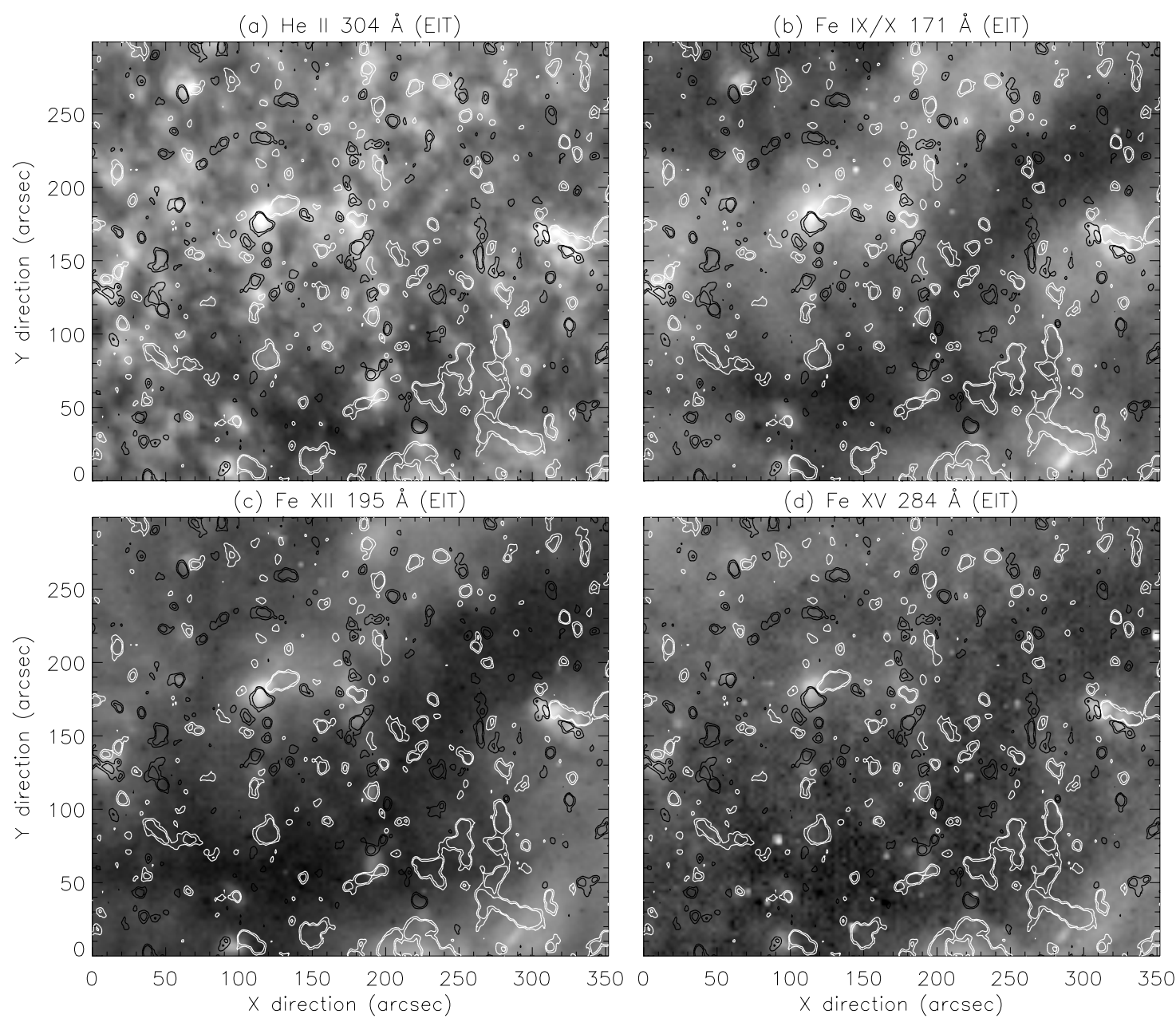

Figure 5.13: $C H 13$ seen in the EIT $304 \AA, 171 \AA, 195 \AA$ and $284 \AA$ channels. Contour plots of magnetograms observed by NSO/Kitt Peak in the same area are presented in the same way as in Figure 5.11, but as obtained on 3 November. 


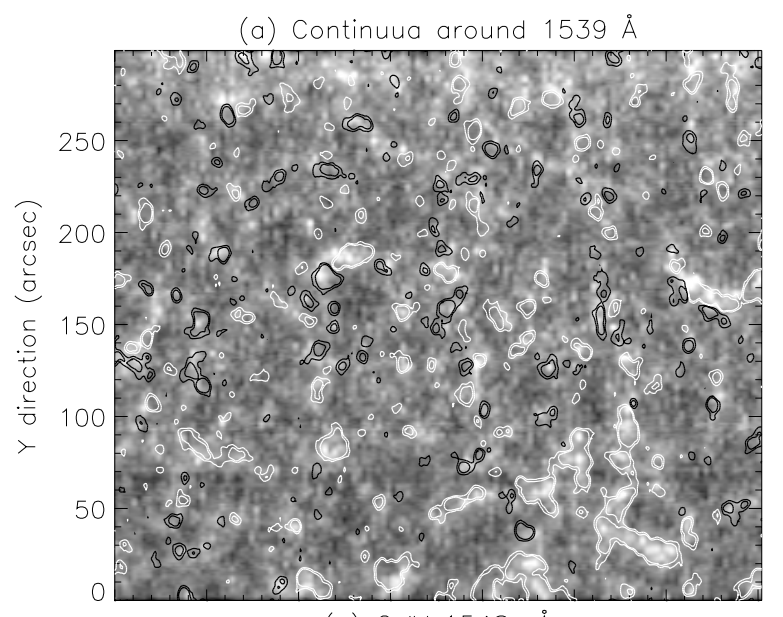

(c) C IV $1548 \AA$

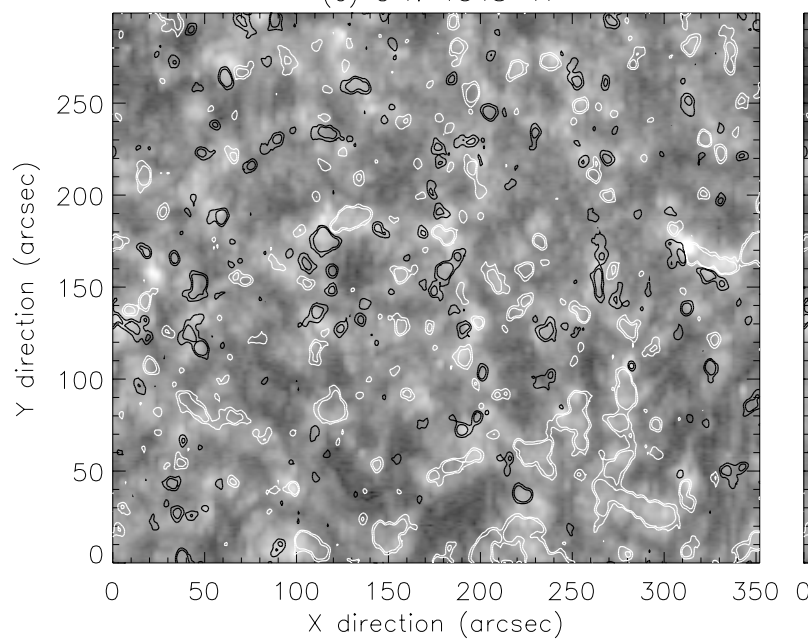

(b) Si II $1533 \AA$

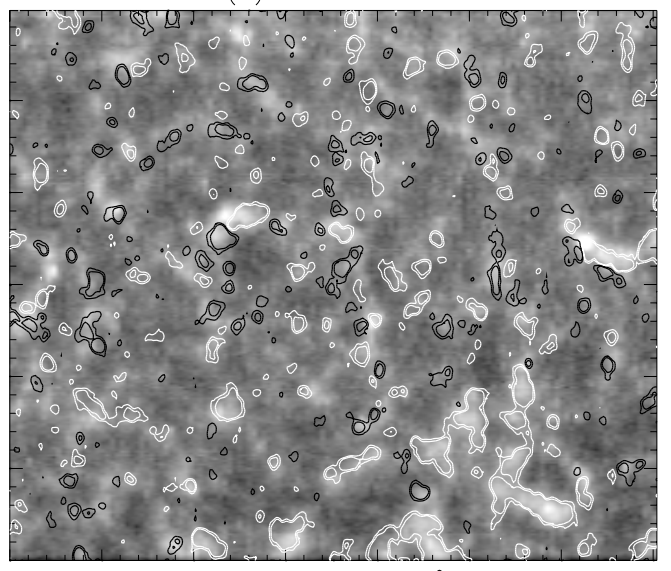

(d) Ne VIII $770 \AA$

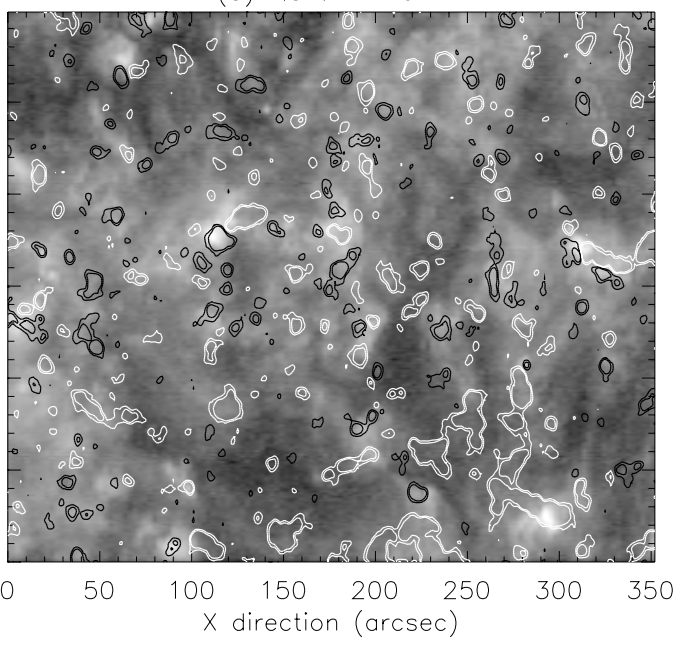

Figure 5.14: $C H 13$ seen in the continuum, Si II (1533 $\AA$ ), C IV (1548 $\AA$ ) and Ne VIII (770 $\AA$ in 2nd order) lines, respectively. Contour plots of magnetograms observed by NSO/Kitt Peak in the same area are presented in the same way as in Figure 5.11, but as obtained on 3 November.

network structure was obviously also changing in shape during this period, but the main features remained unchanged.

\section{Case 2: CH13 observed on 3 November, 1999}

In a previous morphological study of equatorial coronal holes, Wilhelm et al. (2002) found that all ECHs were very clearly defined in the hot lines (Fe XII at $195 \AA$ and Fe $\mathrm{XV}$ at $284 \AA$ ). But some of them, for example, $\mathrm{CH} 13$ observed on 3 November and 7 November, 1999, have much smaller areas if defined in the He II and Fe IX/X channels of EIT and the Ne VIII line observed by SUMER. We discuss here the hole obtained on 3 November, 1999 by inspection of the magnetic field data observed by NSO/KP and ultraviolet images obtained by EIT and SUMER. 
In Figures 5.13 and 5.14, ultraviolet images and magnetic field contours for $\mathrm{CH} 13$ are plotted like in Figure 5.11, but the data were obtained on 3 November. The position of the hole on the full disk EIT image can be seen in Figure 5.10 (left panel), in which the criterion used to determine the boundary of this hole is the same as one for the 5 November hole.

A first impression may be that this hole displays a very different appearance when one compares EIT images in Figure 5.13 with SUMER images in Figure 5.14. However, if they are inspected more carefully, it can be found that the appearance of the hole is very similar as seen in the Ne VIII line and the EIT He II channel. This seems reasonable because they both contain the emission from the transition region.

The contour plots indicate that this hole is occupied predominantly by positive-polarity magnetic field, just like the 5 November hole region. The average net magnetic flux density (signed) is about $1.2 \mathrm{G}$ in FOV of SUMER, much less than that observed on 5 November. There were many small mixed-polarity magnetic features in this $\mathrm{CH}$ area. The negative flux occupied about $40 \%$ of the total unsigned flux in the $\mathrm{CH}$ area, larger than that observed on 5 November.

We may suggest the reasons why the $\mathrm{CH}$ observed on 3 November is very different from that observed on 5 November as follows: First, the average magnetic flux density (signed) observed on 5 November is small. Second, there are many small bipolar magnetic structures present, leading to formation of many small bright structures that dominate the emission observed in lines with lower formation temperatures. But such bright structures eventually disappear with increasing temperature, and have no correspondent emission in the coronal channels of EIT.

\subsection{Outflow at the coronal base}

In this section, we present new observations concerning the source of the fast solar wind, by a direct comparison of the deduced velocity field with the measured photospheric magnetic field in an equatorial coronal hole. The motivation for this work is twofold. First, the previous studies were carried out in polar coronal holes. In this case the line-of-sight geometry is restricted, which may lead to an underestimation of the plasma velocity and greater uncertainty of the magnetic field measurements than at the solar equator. Second, only data from spectral measurements were used in the previous studies. We show here that an analysis combining SUMER spectroscopic data with magnetic field maps can give us a clearer physical picture of the plasma conditions and flow pattern prevailing at the coronal hole base.

In Figure 5.15, we plot the magnetogram together with contours of the Doppler shift of the Ne VIII line (left panel), and the Doppler-shift map together with contours of the intensity of the Ne VIII line (right panel). The velocity field is quite inhomogeneous over the whole field of view (see right panel). Therefore, we smoothed the deduced velocity map over six 

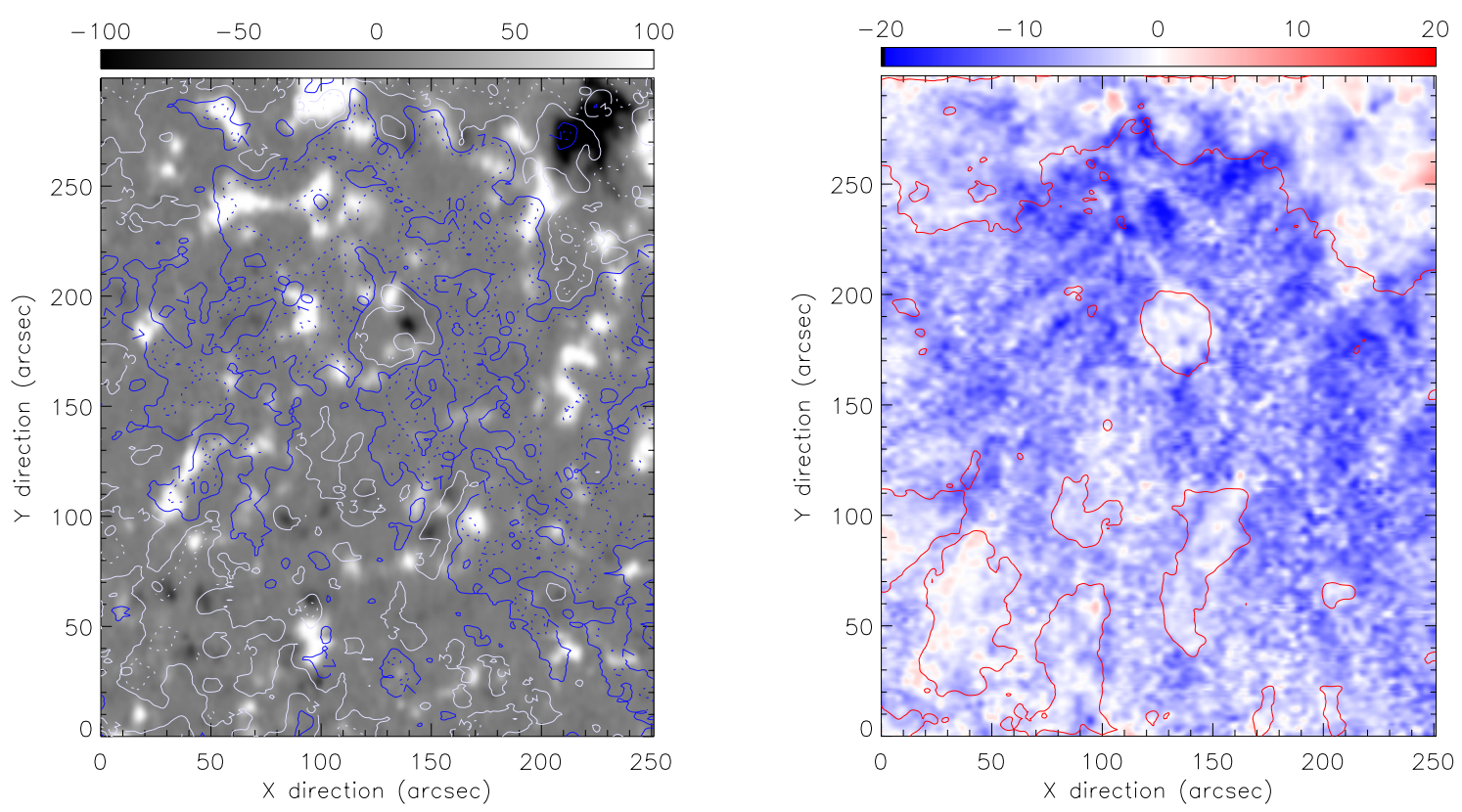

Figure 5.15: Magnetogram and Doppler shift of the Ne VIII line observed on 5 November, 1999. Left panel: Magnetogram (white: positive polarity; black: negative polarity; full scale: $\pm 100 \mathrm{G}$ ) with overlaid contour plots of Doppler shifts $\left(\mathrm{km} \mathrm{s}^{-1}\right.$, with the cool line $C$ I (1542 $\left.\AA\right)$ as the reference line for the Ne VIII line (blue dotted: 10, blue solid: 7, white solid: 3, and white dotted: $0 \mathrm{~km} \mathrm{~s}^{-1}$ ). Right panel: Doppler shifts (full scale: $\pm 20 \mathrm{~km} \mathrm{~s}^{-1}$ ) of the Ne VIII line with overlaid contour plots of its intensity. Note that the upper part of the image is outside of the coronal hole.

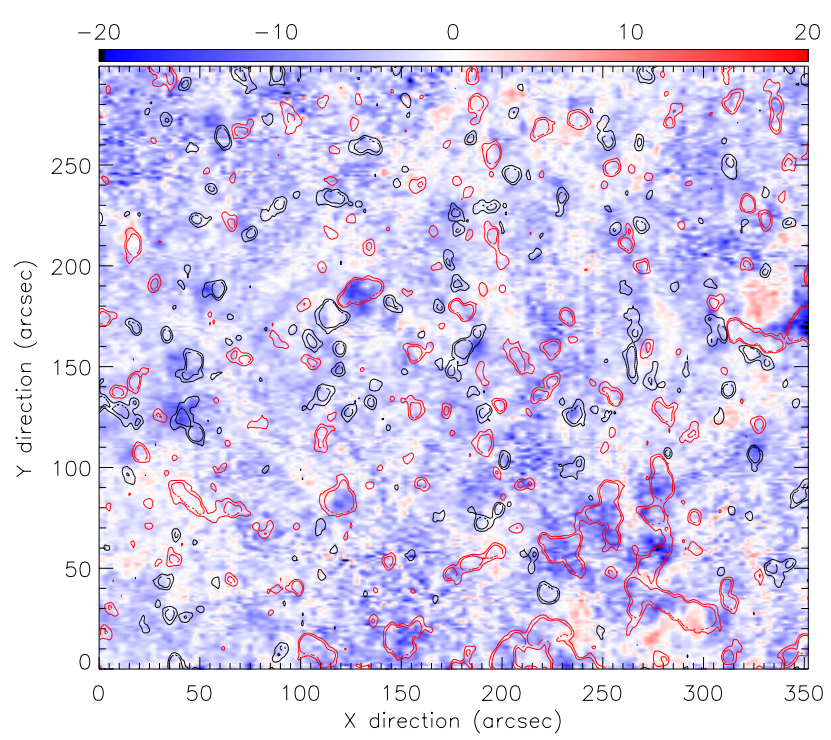

Figure 5.16: Doppler shifts (full scale: $\pm 20 \mathrm{~km} \mathrm{~s}^{-1}$ ) of the Ne VIII line observed on 3 November, 1999 ( $C H 13$ ), overlaid by contour plots of the photospheric magnetic field observed by NSO/KP with contour levels of $-20 \mathrm{G},-10 \mathrm{G}, 10 \mathrm{G}$ and $20 \mathrm{G}$ (red: positive polarity; black: negative polarity). 
adjacent pixels, before the contours of the Doppler shift were actually plotted, in order to show its distribution more clearly. The observed features of this $\mathrm{CH}$ can be divided into three groups: small active regions outside the hole (upper part of the image), and bright and dark regions inside the hole. In order to separate these three regions, we first deduced the frequency distribution of the intensity of the Ne VIII line across the whole image (spectroheliogram). This distribution can be considered to be roughly composed of two components: one contributed by dark regions and another with high intensities by bright regions inside and outside the hole. A threshold of the intensity was then determined, by help of which the boundaries of the three regions are marked by plotting contours (see right panel). The line is predominantly blue shifted in the observed area, and its average blue shift in relation to the $\mathrm{C}$ I line $\left(1542.177 \AA\right.$ ) is found to be $3 \mathrm{~km} \mathrm{~s}^{-1}$ outside the hole, $4 \mathrm{~km} \mathrm{~s}^{-1}$ in the bright region and $7.5 \mathrm{~km} \mathrm{~s}^{-1}$ in the dark region.

The contours of the Doppler shift show a close relationship with the magnetic network structure inside the hole. Larger blue shifts with speed above $10 \mathrm{~km} \mathrm{~s}^{-1}$ (blue dotted line) are mainly associated with regions where the magnetic field is concentrated in a single polarity (positive). As a simple statistical result, the net signed flux density of the underlying field is estimated to be about $11 \mathrm{G}, 8 \mathrm{G}$ and $4 \mathrm{G}$ for blue shifts above $10 \mathrm{~km}$ $\mathrm{s}^{-1}$, between 10 and $7 \mathrm{~km} \mathrm{~s}^{-1}$ and between 7 and $3 \mathrm{~km} \mathrm{~s}^{-1}$, respectively. On the other hand, smaller shifts with speeds below $3 \mathrm{~km} \mathrm{~s}^{-1}$ (white solid line) occur mainly in the dispersed mixed-polarity network, which is also associated with the bright points seen in the Ne VIII line.

The observational results are in agreement with the notion that the fast solar wind is initially accelerated in open magnetic funnels. The measured outflow velocity in the central region of the network is comparable with the model prediction. Some modelling work has been done to study the physical properties of a coronal funnel. Coronal heating and acceleration was considered as being due to cyclotron damping of Alfvén waves and thrusting by Alfvén wave pressure (e.g., Marsch and Tu, 1997; Hackenberg et al., 2000; Li, 2002; Vocks and Marsch, 2002). The resulting plasma properties depend largely on the geometry of the funnel and the details of the wave energy transport and dissipation process. According to these studies, the plasma can be driven to a flow speed of several tens of $\mathrm{km}$ $\mathrm{s}^{-1}$, essentially by a large thermal pressure gradient at the base of the funnel. This pressure gradient results from the quick expansion of the magnetic flux tube, causing rapid heating with height through the high-frequency wave sweeping (resonant absorption).

In section 5.5, the difference between this coronal hole observed on 5 November and on 3 November has been discussed. We show here the Doppler shifts of the Ne VIII line of the 3 November hole area in Figure 5.16. Again, we confirm the results obtained from the 5 November data, i.e., the large blue shifts come from the locations of the unipolar magnetic network. In fact, if only the dark regions are considered, the blue shifts are similar for both datasets. However, large blue shifts seen in Figure 5.16 are more confined in the unipolar magnetic network. The possible reason is that the emission in the open funnels are partly contaminated by the low-lying bright structures. It is also possible that the expansion factor of the coronal funnel is smaller at the height where the Ne VIII line 
is formed, if bipolar magnetic structures are present nearby.

Finally, the results deduced from the 5 November data can be used to estimate the possible contribution of bright points to the total outward mass flux, under the assumption that the deduced Doppler shift of the Ne VIII line represents the real outflow velocity of the $\mathrm{Ne}^{7+}$ ions which are markers of the proton flow. Then we have,

$$
f_{t o t}=f_{b p}+f_{d r}=N_{e, b p} v_{b p} A_{b p}+N_{e, d r} v_{d r} A_{d r},
$$

where $f, v$ and $A$ are the mass flux, deduced outflow velocity and flux tube area. $N_{e, b p}$ and $N_{e, d r}$ denote the electron density in bright points and dark regions. Their ratio can be estimated by the line intensity ratio, if we simply assume the emission volume (having the same bottom area) is the same for a given line in bright points and dark regions of the hole. Thus, we find that

$$
\frac{N_{e, b p}}{N_{e, d r}} \approx \sqrt{\frac{I_{b p}}{I_{d r}}} \approx 1.8 .
$$

The mass flux contributed by bright points to the total outflow within the measured hole area, $f_{b p} / f_{t o t}$, is then estimated to be about $11 \%$. It should be mentioned that we have subtracted a value of $1.5 \mathrm{~km} \mathrm{~s}^{-1}$ from the deduced velocity due to the average red shift of the C I line itself. This estimate of the mass flux by measuring the Doppler shift is consistent with the previous estimate obtained for polar plumes by Wang (1994), and suggests that the portion of the mass flux contributed by bright points to the fast solar wind is at most comparable to the areal ratio of the BPs to the $\mathrm{CH}$ and thus negligible.

\subsection{Coronal holes seen in the $\mathrm{O}_{\mathrm{vI}}$ and $\mathrm{H}_{\mathrm{I}} \mathrm{L} \beta$ lines}

In this section, we further report some interesting results observed in the SUMER spectral window around $1030 \AA$. This spectral window includes very strong emission lines such as the H I L $\beta$ (1025 $\AA$ ), two C II lines (1036 $\AA$ and $1037 \AA$ ) and two O VI lines (1032 $\AA$ and $1038 \AA$ ). They can thus be used to study the detailed morphological properties of the coronal hole, by deducing the line profile parameters reliably in every spatial pixel.

\subsubsection{Analysis of the $\mathrm{H}_{\mathrm{I}} \mathrm{L} \beta$ line}

The general properties in Doppler shifts of various lines have been discussed in Section 5.4.1, in which it has been found that the differences between the very blue and very red shifts of the H I L-11 and He I lines are apparently larger than those of the $\mathrm{O}$ I and $\mathrm{Si}$ II lines. Although these lines reveal effects by opacity, as can be seen by checking their averaged profiles, they show less opacity and exhibit no self-reversal. For an individual spatial pixel, they are found to have a near-Gaussian shape most of the time. Therefore, the Doppler shift deduced from them can be considered statistically reliable. Unlike the H I L-11, the line profile of the H I L $\beta$ appears to have a non-Gaussian shape with a much 

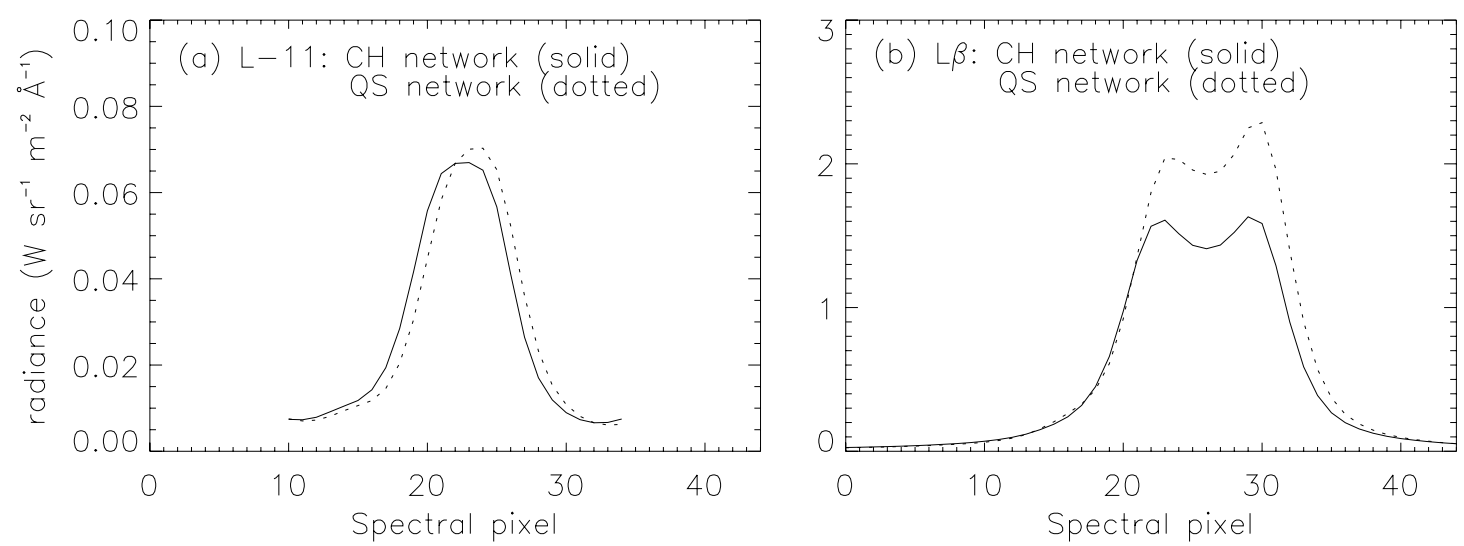

Figure 5.17: Line profiles of the $H$ I $L-11$ and $H$ I $L \beta$ lines in $C H$ and $Q S$ regions. (a) $L-11$; (b) $L \beta$. The data were obtained in $\mathrm{CH} 6$ and QS1.
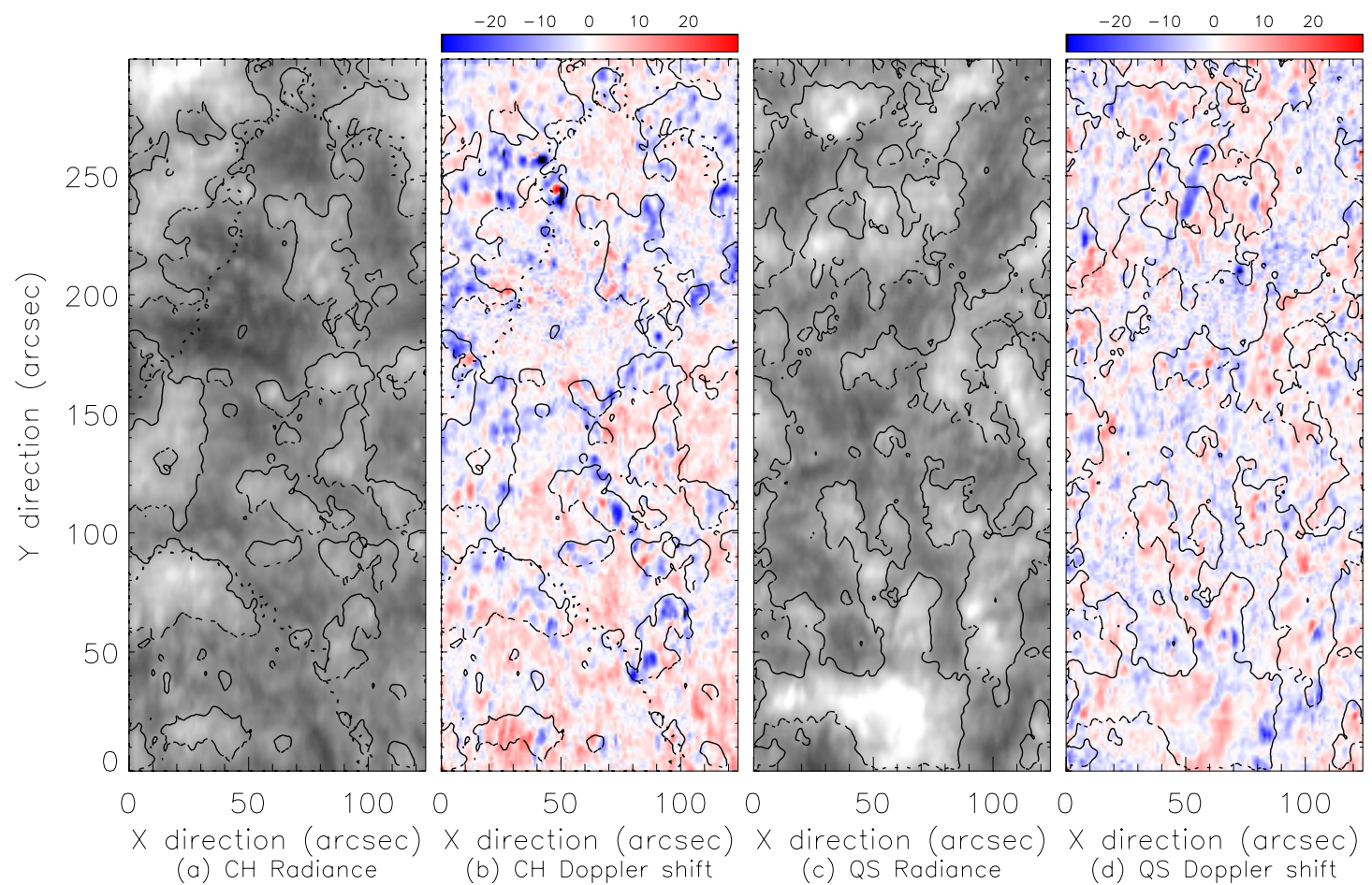

Figure 5.18: Images in intensity and Doppler shift of the $H$ I $L \beta$ line in $C H$ and QS regions. (a) Intensity in $\mathrm{CH}$ (b) Doppler shift in $\mathrm{CH}$; (c) Intensity in QS; (d) Doppler shift in QS. Overlaid contour plots indicate the network determined by the continuum observed simultaneously. Both regions were observed on 11 March, 1999 (CH[ and QS1).

deeper self-absorption reversal around the line center (see Figure 5.17). In Figure 5.17, the profiles of these two lines, which are averaged over the network region, are plotted for both the coronal hole and quiet Sun. It can be found that $\mathrm{L} \beta$ has a slight red asymmetry (with the red peak higher than the blue one) for the quiet Sun, but the asymmetry is less prominent for the coronal hole. 

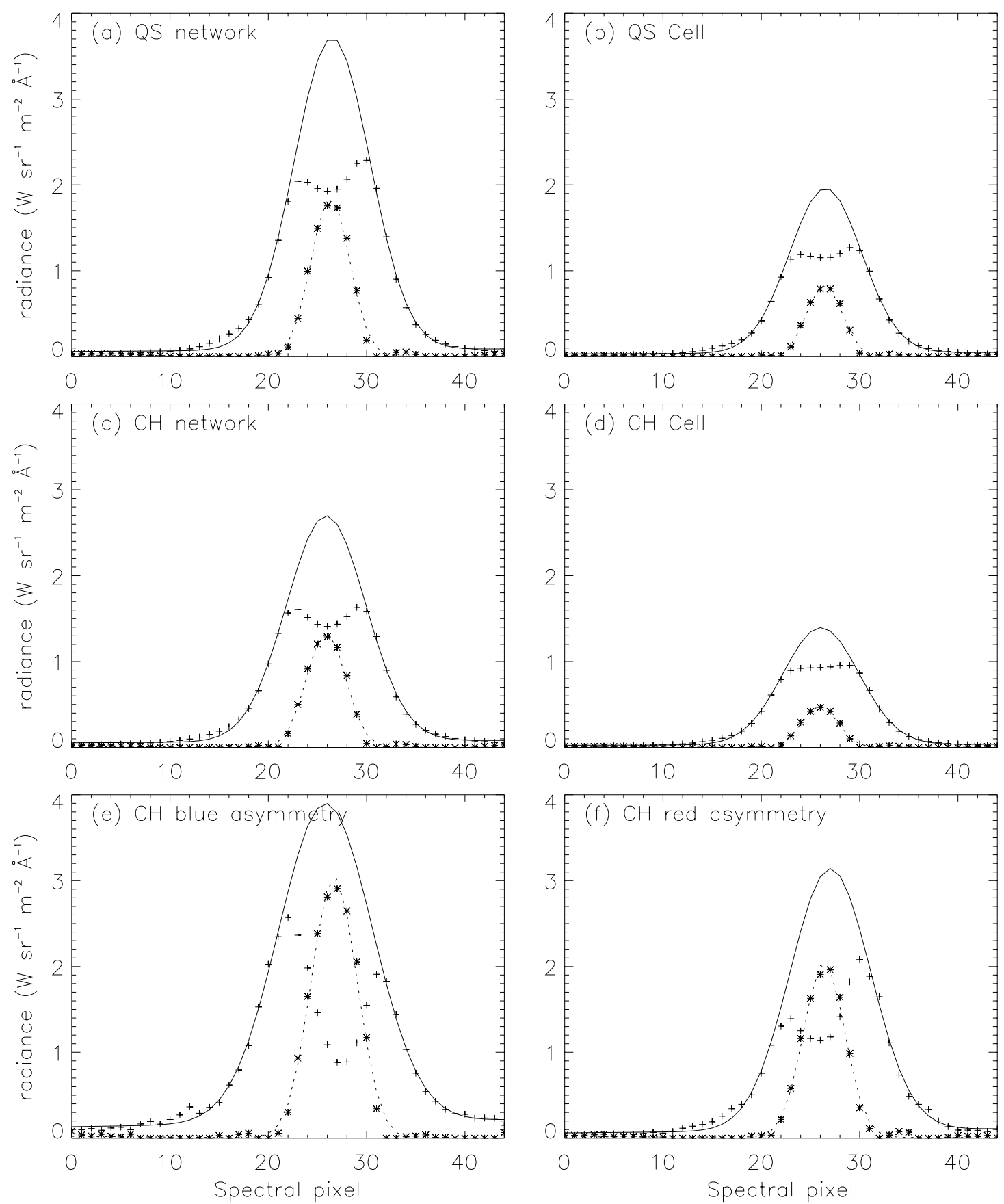

Figure 5.19: Line profiles of the $H$ I $L \beta$ in $C H$ and QS regions. (a) Averaged in QS network; (b) Averaged in QS cell; (c) Averaged in CH network; (d) Averaged in CH cell; (e) Blue asymmetry in $\mathrm{CH}$ network; and (f) Red asymmetry in $\mathrm{CH}$ network. The symbol "+" denotes the observed profiles, and solid lines indicate the profiles fitted from the line wings. The deduced profiles of selfreversal are plotted as symbol "**", and the dotted lines represents such profiles fitted by Gaussian. Both regions were observed on 11 March, 1999 (CH6) and Q\$1). 
It is not straightforward to derive the line shift of $\mathrm{L} \beta$ due to its non-Gaussian shape. In this study, we first define the central position of the line, as the position of the $50 \%$ value of integrated intensity over the whole line (see discussion in 4.4), and then deduce its Dopplergrams, which are shown in Figure 5.18. From Figure 5.18, our data obtained in coronal holes show a striking feature: there are many very blue patches in the Dopplergram of this line (often with a shift of more than $20 \mathrm{~km} \mathrm{~s}^{-1}$ relative to the average line position), while in the quiet Sun this feature is less prominent. These blue patches are located mostly between the boundary of the network and the cell interior. A question that should be naturally asked is whether the very large blue shifts represent real outflow of the plasma.

Inspection of the detailed line profiles from the very blue to very red regions shows that a strong asymmetry of the line profile can be always found. A large blue shift always corresponds to a blue asymmetry (skewed towards the blue side), while a large red shift arises from a red asymmetry. In Figure 5.19 (e) and (f), we show two examples with respect to such situations (symbol "+").

As was mentioned in Section 5.2, theoretical models suggest that the reversals (core) of the $\mathrm{L} \beta$ line are formed in the upper chromosphere and low transition region, while the wings in the lower chromosphere. Because the chromosphere is highly dynamic, it should not be surprising that there exist bulk motions of the plasma where this line is formed, and that such motions may have a gradient in velocity over the line formation height. In this context, an asymmetry of the Lyman lines of hydrogen would be related to large-scale differential motions of the atmosphere (Gouttebroze et al., 1978; Barsi et al., 1979; Schmieder et al., 1998). For example, Schmieder et al. (1998) studied the filament observations with SUMER and CDS. In their work, they found the relationship between the Doppler shift of the He I line and the asymmetry of the H I L-5 line, which are observed by CDS in filaments. The results reveal that in the region of He I blue shifts, the L-5 has red asymmetry, and vice versa. Moreover, two methods were used to estimate the line position of Lyman lines: first by averaging the positions of two peaks, and second by comparing the position of the individual line relative to the center of the profile averaged over the whole observed area. However, they pointed out that a quantitative understanding of the shifts of the Lyman lines in an optically thick plasma can only be achieved by studying the non-LTE (local thermodynamic equilibrium) formation of the line core, where so called partial-redistribution (PRD) scattering physics must be used.

However, previous studies only considered the relative motions between different height of the formation layer. If there is a large-scale motion at the height where the line wings are formed, a shift of the global line profile would also exist. In this study, we will derive respectively the shifts of the global profile and the reversal core by simply using Gaussian fits for an approximate approach. In the fitting procedure, we first set a weight of zero to the central part of the line profile (reversal part), so that the line parameters are essentially determined by the line wings outside of the reversal part. After getting the fitted profile (solid lines in Figure 5.19), which is defined here as the global profile, the reversal part is derived by subtracting the observed profile from the fitted one (dotted lines in 
Figure 5.19). The resulting line fit parameters are listed in Table 5.1.

Table 5.1: Derived line parameters for the H I L $\beta$ line

\begin{tabular}{llccc}
\hline Region & Component & $\begin{array}{c}\text { Peak Intensity } \\
\left(\mathrm{W} \mathrm{sr}^{-1} \mathrm{~m}^{-2} \AA^{-1}\right)\end{array}$ & $\begin{array}{c}\text { FWHM } \\
(\text { pixel })\end{array}$ & $\begin{array}{c}\text { Relative shift* } \\
\left(\mathrm{km} \mathrm{s}^{-1}\right)\end{array}$ \\
\hline QS1 & global & 3.6 & 9.1 & -0.6 \\
network & reversal & 1.9 & 4.6 & -2.9 \\
\hline QS1 & global & 1.9 & 8.7 & 0 \\
cell & reversal & 0.8 & 4.2 & -1.5 \\
\hline CH6 & global & 2.6 & 9.5 & -2.5 \\
network & reversal & 1.3 & 4.6 & -2.4 \\
\hline CH6 & global & 1.4 & 9.1 & 0 \\
cell & reversal & 0.5 & 4.2 & -1.6 \\
\hline CH6 blue & global & 3.7 & 11.2 & -3.2 \\
asymmetry & reversal & 3.0 & 5.5 & 8.1 \\
\hline CH6 red & global & 3.0 & 9.6 & 12.3 \\
asymmetry & reversal & 2.0 & 4.9 & 4.2 \\
\hline
\end{tabular}

Remark "*": line shift relative to the cell average of the global profile for each region. Note that a positive shift is red.

From Figure 5.19, it is found that both line wings and reversal core can be fitted approximately by a Gaussian shape, in particular, for the averaged profiles. Deviations can be found mainly in their blue wing possibly due to a blend of the He II line at 1025.25 $\AA$. Some results have been compiled in Table 5.1.

For the averaged profile, a ratio of the peak intensity between the derived global profile and the reversal ranges from 0.35 to 0.55 , while that of the line width (FWHM) is generally around 2 . Thus the ratio of the integrated intensity between the two profiles is estimated to be around $0.15-0.25$. Their line shift is found to be slightly blue relative to the average value of the global profile obtained in cell regions. It is very interesting that the global profile has a blue shift of $2.5 \mathrm{~km} \mathrm{~s}^{-1}$ in the $\mathrm{CH}$ network, while in the QS network this value is only $0.6 \mathrm{~km} \mathrm{~s}^{-1}$. Considering the network may be the source of the nascent fast solar wind, this result is indeed expected by us. However, this conclusion should be confirmed by analyzing more data in the future.

For the strongly asymmetric profiles, a larger departure of the line position between the derived global and reversal profile can be found, with a value of about $\pm 10 \mathrm{~km} \mathrm{~s}^{-1}$. Compared with the average line position of the global profile in the cell region, the one deduced from the asymmetric profiles are found to be blue shifted by $3.2 \mathrm{~km} \mathrm{~s}^{-1}$ for the blue asymmetry and red shifted by $12.3 \mathrm{~km} \mathrm{~s}^{-1}$ for the red asymmetry, while the values of the reversal profile are $8.1 \mathrm{~km} \mathrm{~s}^{-1}$ and $4.2 \mathrm{~km} \mathrm{~s}^{-1}$, respectively, and both are red shifts. On the other hand, the blue asymmetry results in a blue shift of about $27 \mathrm{~km} \mathrm{~s}^{-1}$ in the 
old Dopplergram as shown in Figure 5.18, while the red asymmetry corresponds to 24 $\mathrm{km} \mathrm{s}^{-1}$ red shift. These previous values are much larger than those deduced by using the current method. We may conclude that the very blue patches, for the $\mathrm{CH}$ data shown in Figure 5.18, indicate strong blue asymmetries of the $\mathrm{L} \beta$ line, but not real line shifts.

However, the reasons why more blue asymmetries are observed in $\mathrm{CHs}$ are not clear. They are possibly associated with the concentration of the open magnetic field in the $\mathrm{CH}$ network. More observational and theoretical studies seem to be necessary in the future. Hydrogen is the main component of the solar wind, and its behaviour at the base of CHs is still unclear.

Using the current method, we deduce again the Dopplergrams determined by the line wings of the $\mathrm{L} \beta$. The very blue patches disappear, and the range of line shifts is now comparable with those of the L-11 and He I lines. They are used in the following discussion, together with other lines in the same spectral window and the MDI magnetograms.

\subsubsection{Loop-like structures seen in the $O$ vi line}

In order to analyze more carefully the relationship between the transition region structures and the underlying chromosphere, we continue to study the morphology of the coronal holes observed in the spectral window around $1030 \AA$. The images in intensity and Doppler shift, including the H I L $\beta, \mathrm{C}$ II and O VI lines, are plotted in Figures 5.20 and 5.21, which were obtained in $\mathrm{CH} 13$ and $\mathrm{CH} 6$, respectively. Meanwhile, the photospheric magnetic field observed by MDI at the same time is also given in each figure. All figures are overlaid by contours of the continuum emission observed simultaneously in order to outline the network region ( $\approx 33 \%$ of the $\mathrm{CH}$ area is occupied by the network). The spectroheliograms shown in Figures 5.20 and 5.21 have been processed with an increased contrast of the intensity in order to display the loops more clearly. The Doppler shifts of the $\mathrm{C}$ II and $\mathrm{O}$ VI lines have been calibrated relative to the chromospheric lines of $\mathrm{O}$ I, while the $\mathrm{L} \beta$ line is referenced to its average position.

From the upper panels of Figures 5.20 and 5.21, we confirm again that the network is dominated by loop-like structures in the transition region, as found in the quiet Sun (Warren and Winebarger, 2000; Feldman et al., 2001). Such structures are very clear when seen in the emission of the Li-like ion $\mathrm{O}^{5+}$. However, they can also be identified in other lines, such as $\mathrm{C}$ II and $\mathrm{H} \mathrm{I} \mathrm{L} \beta$, but with lower contrast in brightness compared with the surrounding area. In addition, all the structures seen in these lines are very different from those seen in the continuum, which appears as small bright patches outlining the cell boundaries.

Comparing the network structure outlined by continuum emission with the magnetogram, one can readily find that the network seen in ultraviolet images spatially coincides with the locations, where the photospheric magnetic field flux is concentrated. The network in the two holes shown in Figures 5.20 and 5.21 was dominated by the positive polarity (red color) of the magnetic field. There were also some mixed-polarity features present. As 

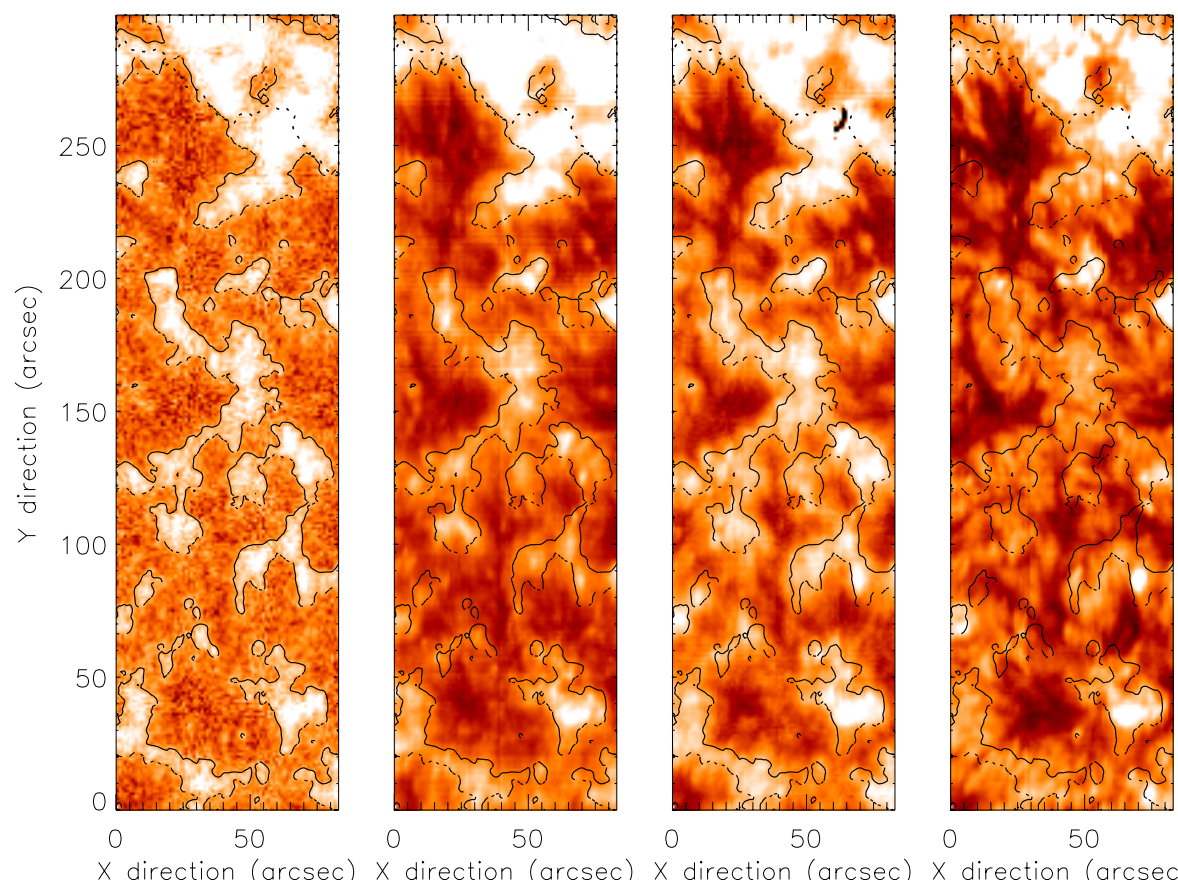

(a) Continuum

(b) $H \perp\llcorner\beta$

(c) $\mathrm{C} \|$

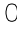

50
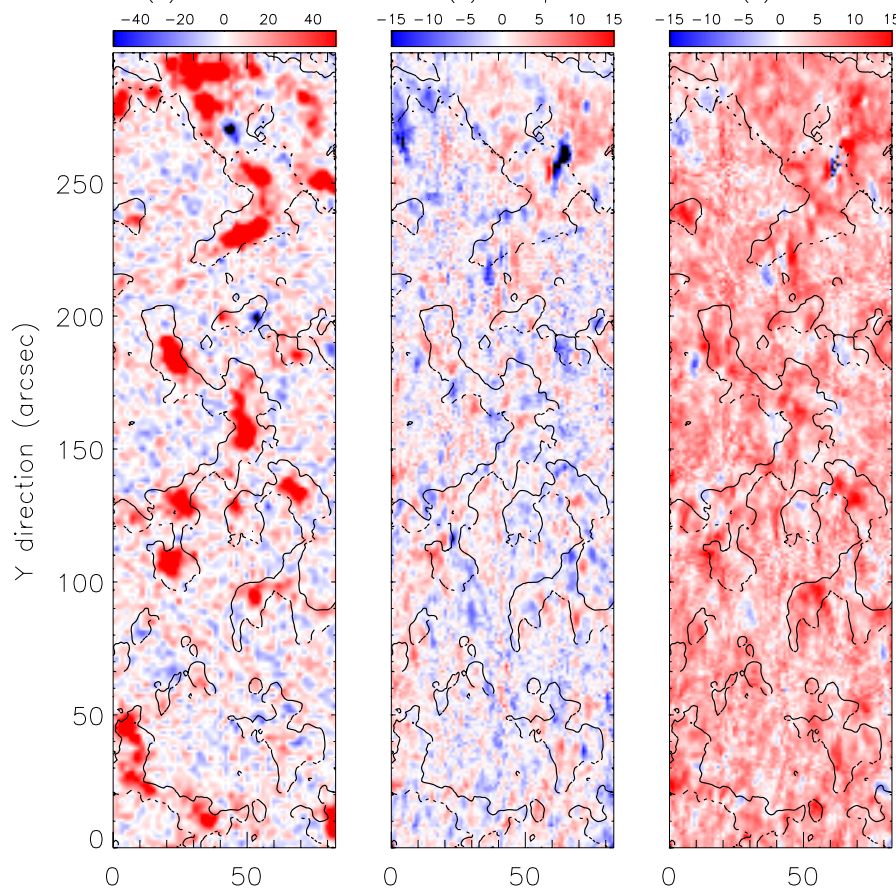

(d) $O \mathrm{VI}$

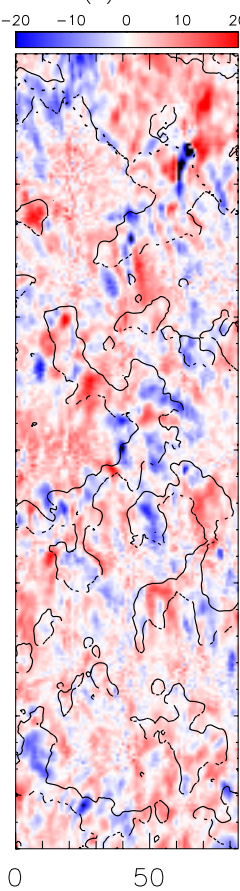

$X$ direction (arcsec)

(e) MDI magnetogram

(f) $H \mid L \beta$

(g) $\mathrm{C} \|$

(h) O VI

Figure 5.20: $\mathrm{CH}$ images in intensity and Doppler shift in the spectral window $1020 \AA$ to 1040 $\AA$. Upper panels: Intensity of (a) continuum; (b) H I L $\beta$ (1025 $\AA$ ); (c) C II (1037 $)$ ); and (d) $O$ VI (1031 A). Bottom panels: (e) MDI magnetogram; and Doppler shift of (f) H I L $\beta$, (g) C II and (h) $\mathrm{O}(\mathrm{VI})$. The $\mathrm{CH}$ was observed on 8 November, 1999 (CH13). 


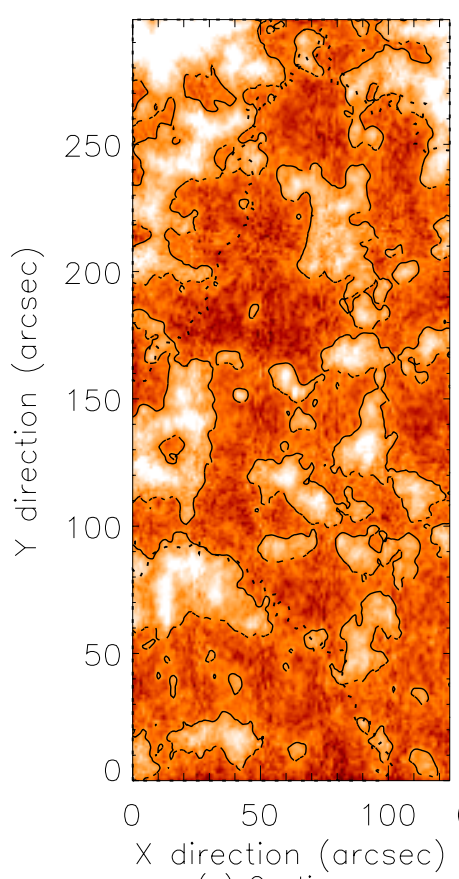

(a) Continuum

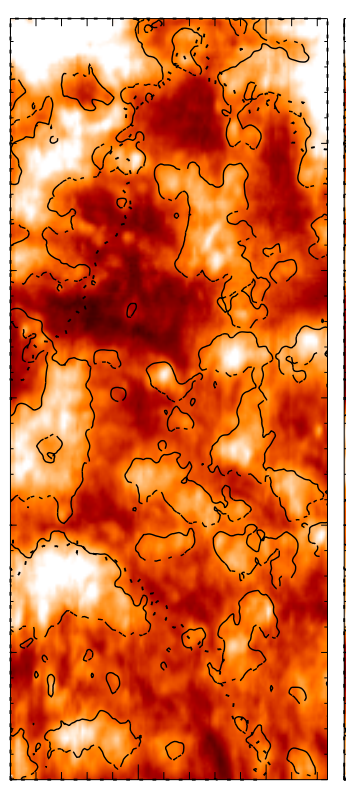

50

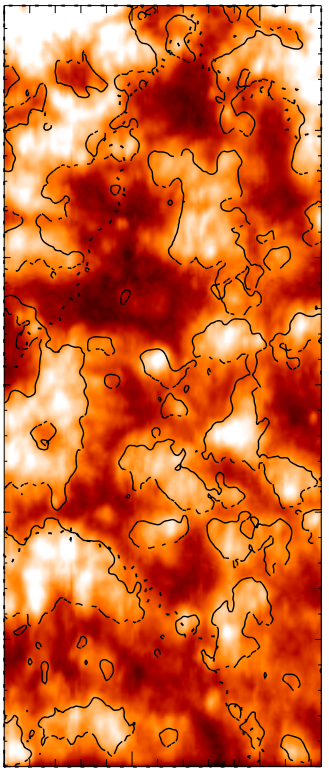

$\begin{array}{lll}50 & 100 & 0\end{array}$

(c) C II

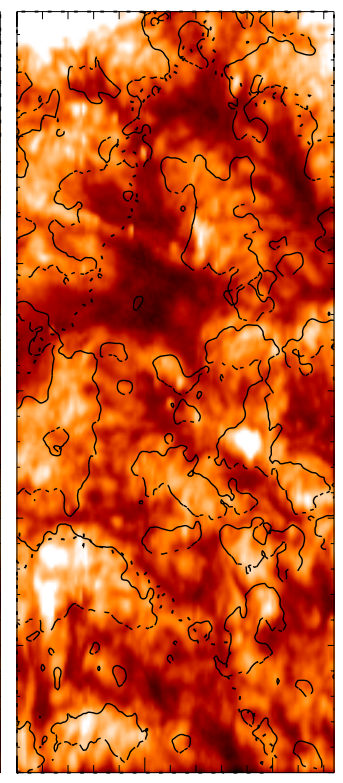

$50 \quad 100$

(d) 0 VI

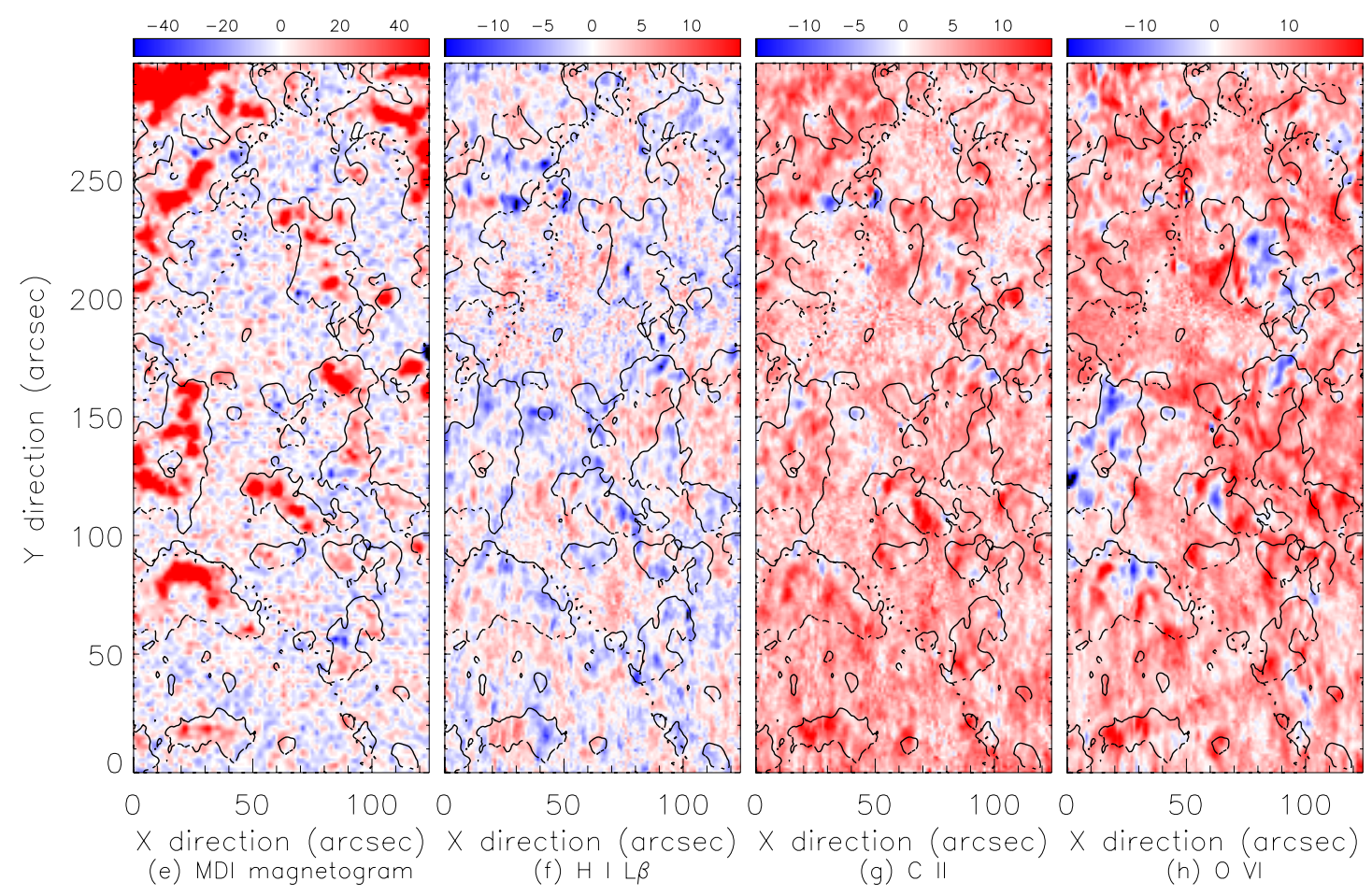

Figure 5.21: $C H$ images in intensity and Doppler shift in the spectral window $1020 \AA$ to 1040 $\AA$. Upper panels: Intensity of (a) continuum; (b) H I L $\beta$ (1025 A); (c) C II (1037 A); and (d) $O$ VI (1031 A). Bottom panels: (e) MDI magnetogram; and Doppler shift of (f) H I L $\beta$, (g) C II and (h) $\mathrm{O}$ (VI). The $\mathrm{CH}$ was observed on 11 March, 1999 (CH6). 
was discussed in the section 5.5. mixed-polarity magnetic features usually correspond to locations with bright points seen in transition region and coronal lines. In these figures, they indeed appear as brighter cores seen in the O VI line. Moreover, the locations of these bright cores spatially coincide with those seen in the continuum and the $\mathrm{H} \mathrm{I} \mathrm{L} \beta$ and C II lines. This implies that such an enhanced network emission is very likely caused by the interaction of the closed magnetic loops with the unipolar fields in the coronal hole.

For most bright network structures in the hole, however, one finds that there is no visible stronger magnetic flux with the opposite polarity nearby. By inspection of the spectroheliograms, many loop-like structures seem to have a footpoint rooted in the intranetwork
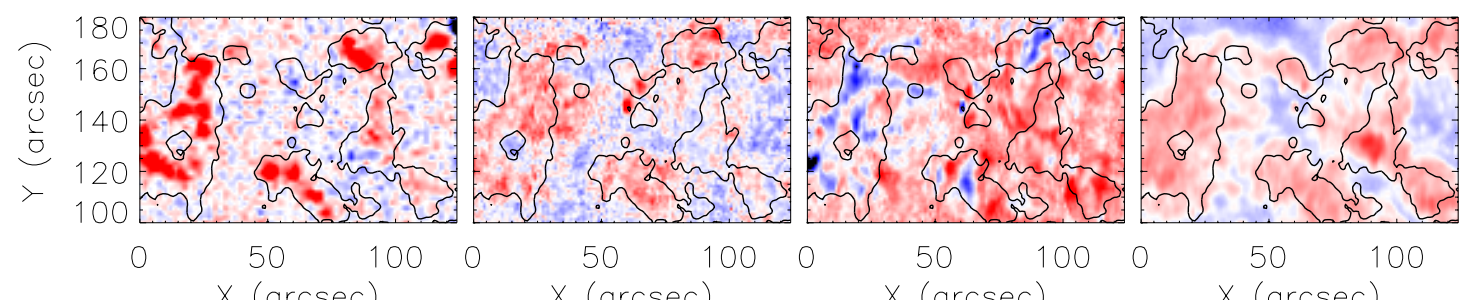

$X(\operatorname{arcsec})$
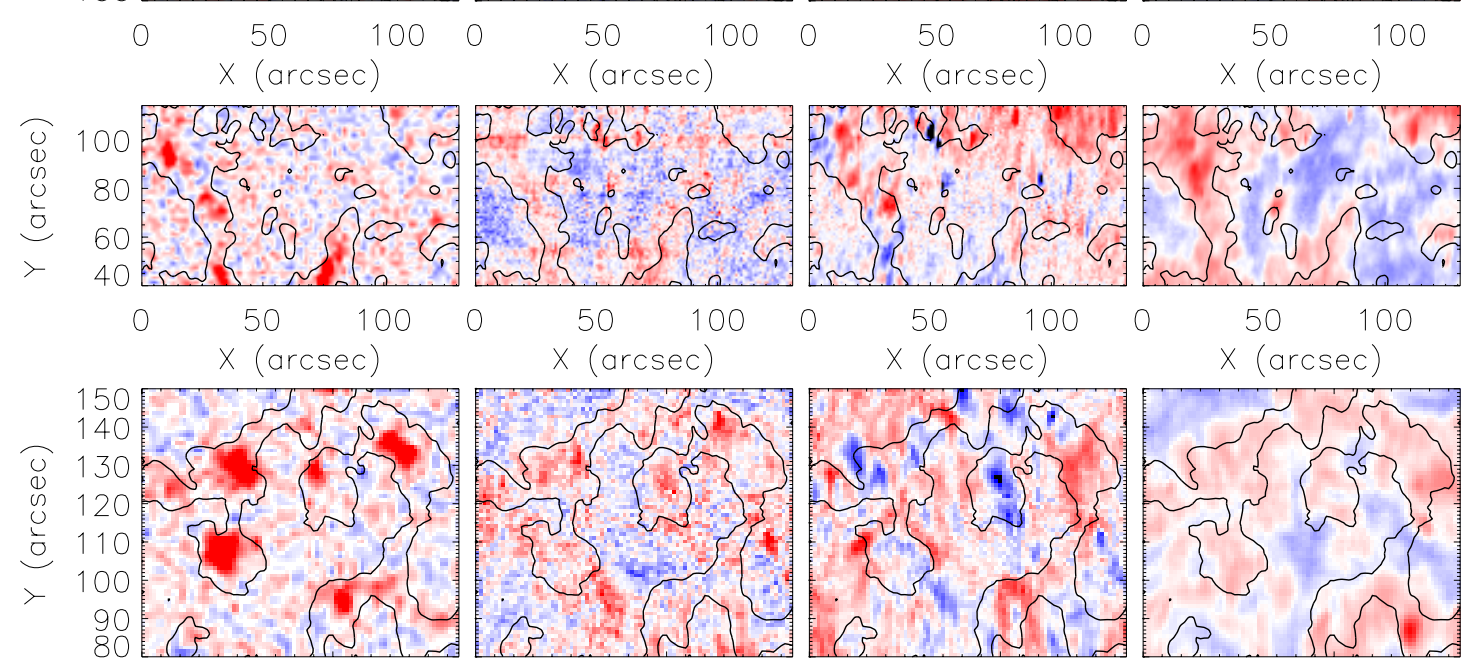

100

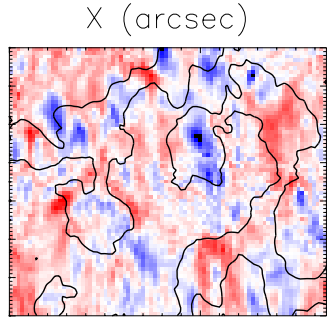

$x(\operatorname{arcsec})$

$0 \quad 50$

$0 \quad 50$
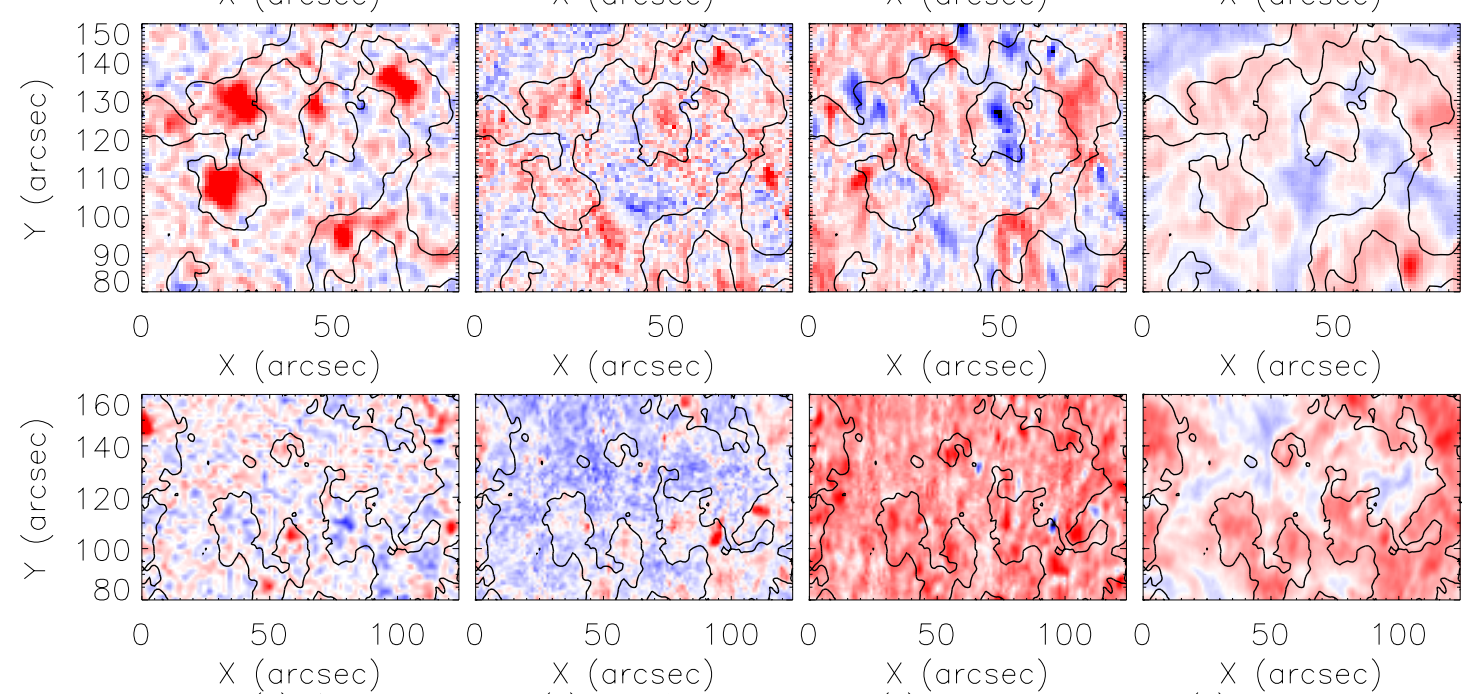

$x(\operatorname{arcsec})$
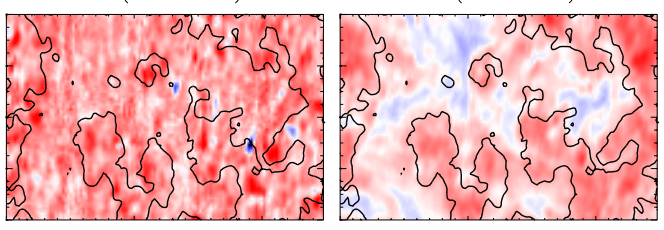

(a) MDI

(b) O VI Width
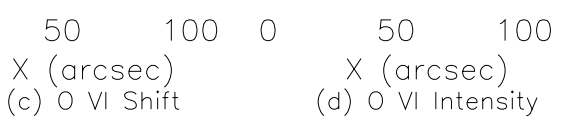

(d) O VI Intensity

Figure 5.22: Fine structures in $C H$ and $Q S$ regions seen in the $O$ VI line. (a) MDI magnetogram; (b) Line width; (c) Doppler shift; and (d) Intensity. From top to bottom: CH6 on 11 March, 1999; CH11 on 19 October, 1999; CH13 on 8 November, 1999; and QS1 on 11 March, 1999. The positive magnetic field is plotted in red color, while the negative in blue. For the intensity and line width, red color represents larger values. Note that the images are cuts from the SUMER full images obtained by slit scanning. 
and extend into the cell interiors. Some of them appear as clusters with a star-like shape (see further in Figure 5.22). Moreover, their visible footpoints apparently correspond to the bright patches seen in chromospheric emissions. This result seems to be different from that observed in the quiet Sun by Feldman et al. (2001), as was mentioned above. They found that most of the loops straddle the chromospheric network and have no visible connections with bright patches seen in chromospheric lines. The possible interpretation is that such a difference may be due to the different geometry of the magnetic field in the two regions. Unfortunately, for the data obtained from the QS region in our study, we are unable to distinguish the footpoints of the loop-like structures due to the low contrast in intensity, though such structures are also present. However, more data need to be analyzed to confirm both results.

In the QS region, Warren and Winebarger (2000) also found that most of such structures had no connection with the intranetwork magnetic field having mixed polarities as found by Dowdy (1993). Hence, our inspection of heliograms of the O VI line, by co-aligning the MDI magnetogram with the SUMER image, confirms again their results. As a possible explanation, they suggested that these fine features may be related to small loops connected to the internetwork (cell interiors) magnetic field or highly dynamic structures, such as spicules, which are not really loops. As was pointed out by them, however, the visible structures observed above the limb are dominated by EUV spicules, which appear nearly straight, but not as loops (also see, Wilhelm, 2000). The possible solution of this difficulty may be to consider that both structures (loops and spicules) are present, both anchored in the network. On the one hand, the small loops are less extended in height (less then $10^{\prime \prime}$ ) and can not be distinguished if observed above the limb due to considerable overlapping of such loops (Warren and Winebarger, 2000). On the other hand, EUV spicules extend much higher (above $10^{\prime \prime}$ ), but can not readily be distinguished from the loops on the disk, due to either their projection effect or their overlapping with low-lying magnetic structures. As we will discuss in the following, such a situation may really be true in the coronal holes.

According to previous empirical studies of the network (Dowdy et al., 1986, Dowdy, 1993 ), it is believed to consist of both low-lying loops (less then $10^{4} \mathrm{~km}$ ) and largescale magnetic funnels being open into the corona. Very recently, a model sketch of the transition region structure has been suggested by Peter (2000, 2001), who studied several components of the transition region lines observed by SUMER in the quiet Sun, and found that a presence of the second broader spectral component is a general feature of transition region lines. This second component, with a broader and weaker profile, is suggested to originate from the large loops in the quiet Sun or open funnels in the coronal hole, while the core (first component) is from the smaller loops, and has a narrower line width and stronger intensity. For the O VI line, he found this second component contributed about $14 \%$ to the total line emission and was more redshifted by $3 \mathrm{~km} \mathrm{~s}^{-1}$ with respect to the second one.

Let us now return to our observations. In Figure 5.22, we show segments of the full images obtained from observations of $\mathrm{CH} 6$ on 11 March, 1999; $\mathrm{CH} 11$ on 19 October, 1999; 
$\mathrm{CH} 13$ on 8 November, 1999; and QS1 on 11 March, 1999. The MDI magnetograms and the intensity, line width and Doppler shift of the O VI line are plotted. From the Dopplergrams, one finds that fine structures with large blue shifts are also present. Some of these structures appear as loop-like and spatially coincide with the locations of the loop-like ones seen in the intensity image, while some appear as blue points with less extension and the locations corresponding to the footpoints of the intensity loop-like structures. The blue structures usually have a broader line width. Like the Ne VIII line discussed in section 5.6, they tend to be present above the concentration of a large unipolar magnetic field without obvious bipolar magnetic features nearby.

One the other hand, structures with a red Doppler shift are always present in the coronal hole seen in this line (actually, the average Doppler shift is still red in this line). Red shifts come from both the cell regions and the network, with a mixed polarity of the magnetic field being present when seen in the MDI magnetogram. Such red-shift structures usually have a low intensity and small line widths, if located in the cell interiors, and have intermediate line widths, if located in the network.

Hence, it can be concluded that blue shifts can be also found in transition region lines (although on average the lines are red shifted) and tend to occur in the network where a large unipolar magnetic field is concentrated. A reasonable inference may thus be made, i.e., that such blue shifts occur mainly in areal sections of the magnetic network, in which the magnetic field is really open to the corona, and are also time dependent as will be discussed in the next section. This conclusion may answer the question why a different blue-red-shift ratio of the C IV line is obtained in different observations, a result which has been discussed in the section 5.4.1.

Furthermore, by inspection of the Dopplergrams of the $\mathrm{H} \mathrm{I} \mathrm{L} \beta, \mathrm{C}$ II and O VI lines, one finds that at those locations where blue shifts are seen in the $\mathrm{O}$ VI line blue shifts are usually also seen in the $\mathrm{L} \beta$ line. For the $\mathrm{C}$ II line, blue shifts appear seldom in Dopplergrams, but if visible, they are of smaller magnitude, the corresponding regions of smaller spatial size.

Finally, the Dopplergrams observed in the QS region reveal very different properties from those of the $\mathrm{CH}$ regions (see bottom panels in Fig. 5.22). It seems that in the $\mathrm{CH}$ regions there is no similar relationship between the structures seen in Dopplergrams and intensity images. However, the line width shows a positive correlation with the intensity, i.e., large line widths are mainly found in the network.

If we conjecture the loop-like structures seen in $\mathrm{CH}$, with a broader line width and blue shift, correspond to a magnetic flux tube that is open to the corona, and that those with a narrower line width and a red shift to cooler magnetic loops that are closed locally. These results are in agreement with those obtained for the quiet Sun by Peter (2000, 2001). Moreover, if it is true that such structures with a blue shift are open to the corona, it is possible that some of them will appear as visible EUV spicules or macrospicules if seen above the limb. According to the SUMER observations by Budnik et al. (1998), EUV spicules have a width of $10^{\prime \prime}-20^{\prime \prime}$ when seen in transition region lines, and disappear at a temperature above $0.5 \mathrm{MK}$, in agreement with observations of the fine structures on the 
disk as found in our study. Spicules and macrospicules have also different orientations observed in $\mathrm{CH}$ regions (see the figures in Wilhelm, 2000; Wilhelm et al., 2000); thus they may have projection on the disk with different lengths.

\subsection{Rapid time variations}

During the solar minimum in October 1996, rapid-time-cadence observations of the equatorial coronal hole $(\mathrm{CH} 3)$ were carried out with exposure times of $20 \mathrm{~s}$ (DS1) and $60 \mathrm{~s}$ (DS2) in the wavelength range between $760 \AA$ and $795 \AA$. Four spectral windows, centred on the N IV (765 ̊), Ne VIII (770 ̊), S V (786 ̊) and O IV (790 ̊) lines, were telemetred. During these observations, no solar rotation compensation was applied and the slit was kept in a fixed position (no raster step), and therefore SUMER "scanned" the Sun with the speed of the solar rotation (about $10^{\prime \prime}$ per hour) along the West-East direction. Slit 4 and 2, both with widths of $1^{\prime \prime}$, were used in DS1 and DS2, respectively. Hence, SUMER needed about 6 min (18 exposures for DS1 and 6 exposures for DS2) to scan a 2" -wide region in West-East direction on the disk. The durations in time of these observations are $2 \mathrm{~h} 55 \mathrm{~m}$ for DS1 and $10 \mathrm{~h} 32 \mathrm{~m}$ for DS2. Therefore, the corresponding effective areas on the disk covered by SUMER during the observations are about $27^{\prime \prime} \times 105^{\prime \prime}(\mathrm{DS} 1)$ and $97^{\prime \prime} \times 300^{\prime \prime}(\mathrm{DS} 2)$, respectively. The intensity and Doppler shift along the slit (Y-direction) versus time (X-direction) are shown in Figures 5.24 and 5.25. Note that only a part of each dataset is plotted (DS1: whole slit and the first 84 min; DS2: 170 px -295 px along the

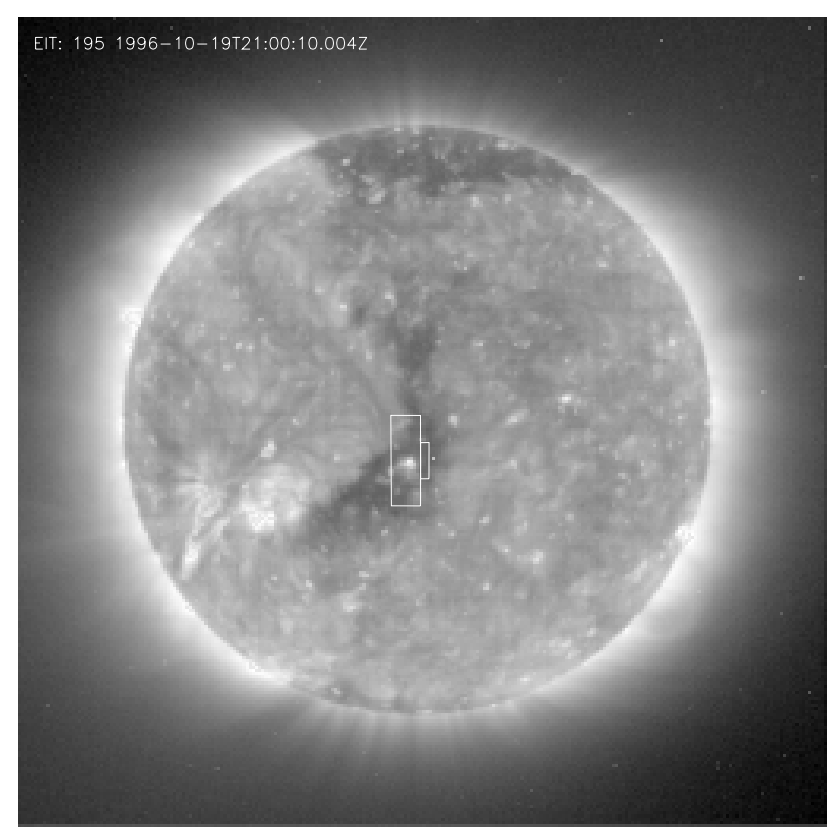

Figure 5.23: An equatorial coronal hole as seen in the $195 \AA$ channel of EIT observed on 19 October, 1996, with the field-of-view of SUMER being superimposed as a white box. 
slit and the first $200 \mathrm{~min}$ ), which was obtained from a deep region in the hole (see Figure 5.23. left half in the right frame window for DS1; upper-right corner in left frame window for DS2).

As will be discussed in the next chapter, the transition region lines such as $\mathrm{N}$ IV and $\mathrm{O}$ IV have on average a red shift of around $5 \mathrm{~km} \mathrm{~s}^{-1}$, while the Ne VIII has a blue shift of around $5 \mathrm{~km} \mathrm{~s}^{-1}$. We can thus make a wavelength calibration with respect to these values for each line. The data gap indicated by white vertical lines in Figure 5.25 is due to a long exposure time of $200 \mathrm{~s}$ used by SUMER during these periods, which are not considered here.

In the quiet Sun, such observations have been made with SUMER to study the oscillatory natures of the solar chromosphere and transition region (see, e.g., Carlsson et al., 1997; Judge et al., 1997; Curdt and Heinzel, 1998; Wikst $\varnothing l$ et al., 2000; Hansteen et al., 2000). In this study, we make no attempt to do such work quantitatively. The main purpose here is to to give a qualitative description of the rapid variations, in both intensity and line shift, as obtained from these high-time-cadence observations in equatorial coronal holes.

Figures 5.24 and 5.25 show that the two transition region lines have a very similar appearance when seen in both intensity and line shift images, which should be not surprising because they have a similar formation temperature. The intensity images shown in these two figures (left panel) indicate the selected hole area was mainly occupied by the network. At a first sight one can find that the two transition region lines display a very dynamic behaviours, while the Ne VIII line appears much smoother and less structured, except for some bright structures. Both temporal and spatial variations in brightness can be clearly seen for the N IV and O IV lines. The temporal variation is here referred to a change of brightness within a period of $6 \mathrm{~min}$. The visible bright structure has a typical size of $5^{\prime \prime}$ to $20^{\prime \prime}$ along the slit, which is indeed falling in the range of the network width.

A clearer pattern of such a dynamic behaviour can be seen in the line shift maps (right panels in Figure 5.24 and Figure 5.25. All three lines analyzed here show a very structured pattern. The two transition region lines have generally red shifts, but blue patches with a small area can always be found, particularly for DS1, which has an exposure time of 20 s. On the other hand, the Ne VIII line is on average blue shifted, but the Dopplergram is still highly structured. The areas with larger blue shifts appear also as blue patches. Such blue patches seen in the Ne VIII line spatially coincide with those seen in the N IV and O IV lines, but have apparently a larger areal extension. In addition, their size along the slit and the location of the structures seen in line shift usually correspond to similar fractures seen in intensity maps. Their durations in time are different. A rapid variation can be recognized to last only for one exposure time (20 s for DS1 and $60 \mathrm{~s}$ for DS2).

The main result from this study that should be emphasized here is that the transition region and coronal base are not "quiet" but very dynamic when observed at high time resolution. Although the Ne VIII line reveals on average a blue shift in the hole, the velocity field of the flow indicated by this measurement shows sizable variations, perhaps indicating very unstable properties of the nascent solar wind when detected at this height. This result is 

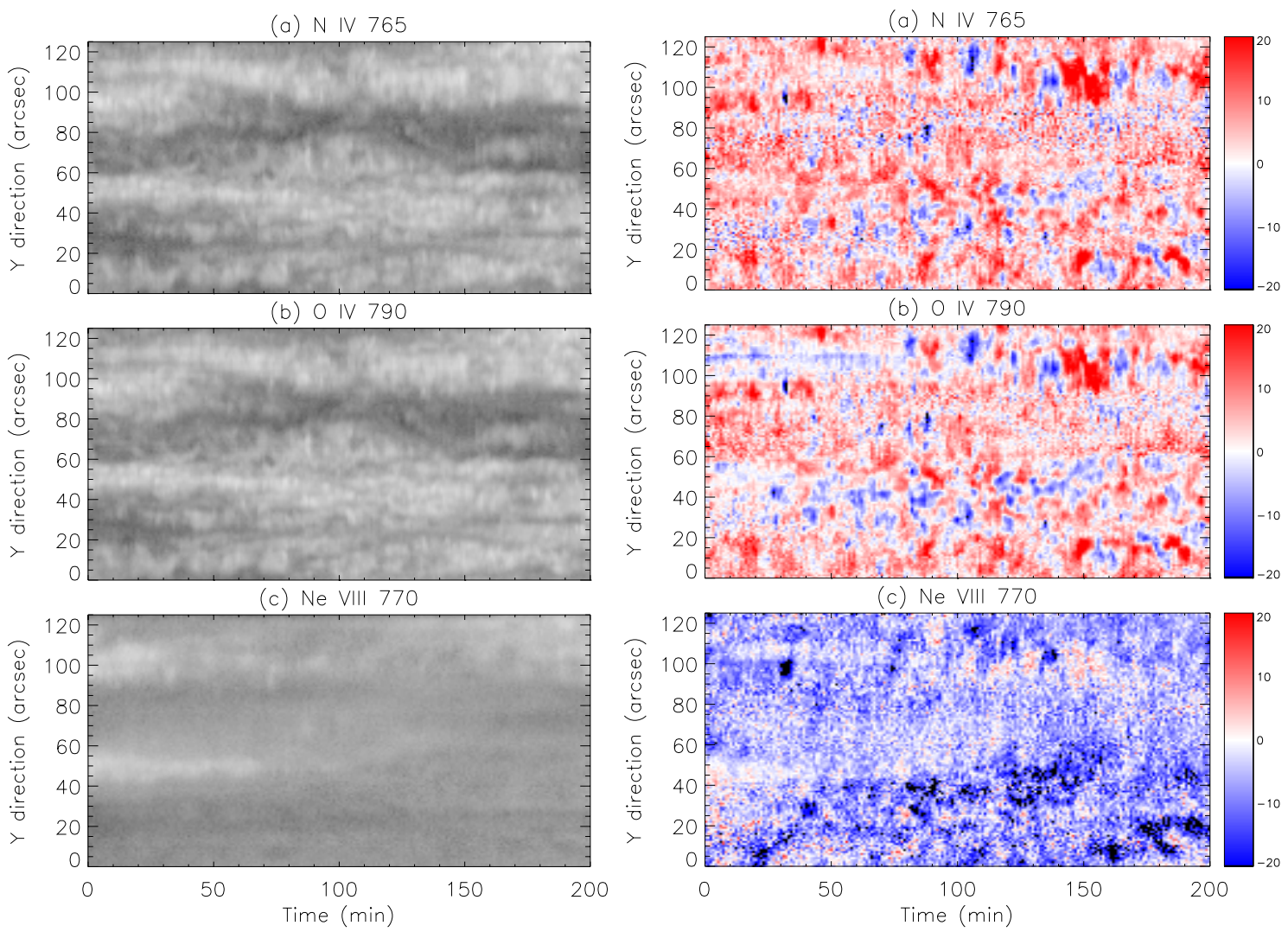

Figure 5.24: Images in intensity (logarithmic scale) and line shift (right frames) in the spectral window from $760 \AA$ to $795 \AA$. (a) N IV (765 $)$ ); (b) O IV (790 ̊) and (c) Ne VIII (770 ̊). An exposure time of $60 \mathrm{~s}$ has been applied.

consistent with the widely accepted conception that very-small-scale magnetic activities may be the ultimate source for heating the corona and accelerating the solar wind.

\subsection{Case study of a transient event}

During the observation of the coronal hole on 12 March, 1999, SUMER captured an interesting transient event in $\mathrm{CH} 6$. This event can be readily distinguished in Figure 5.3 as well as in Figure 5.26 in details, in which there is a prominent bight point in the heliograms of the continuum and the $\mathrm{C}$ I line. In the heliograms of the $\mathrm{N} \mathrm{V}$ line, this location is marked as a black data gap, because this line was broadened in excess of the small spectral window selected for determining the line position. For the $\mathrm{Mg} \mathrm{X}$ line, we are unable to determine the line parameters, due to its weak signature relative to the largely enhanced continuum and other chromospheric lines. The spectra of this event between $1234 \AA$ and $1254 \AA$, together with the normal network spectra in this hole, are given in Figure 5.27 (bottom panel). The upper panel shows the intensity ratio between this event and the normal network of the coronal hole. 

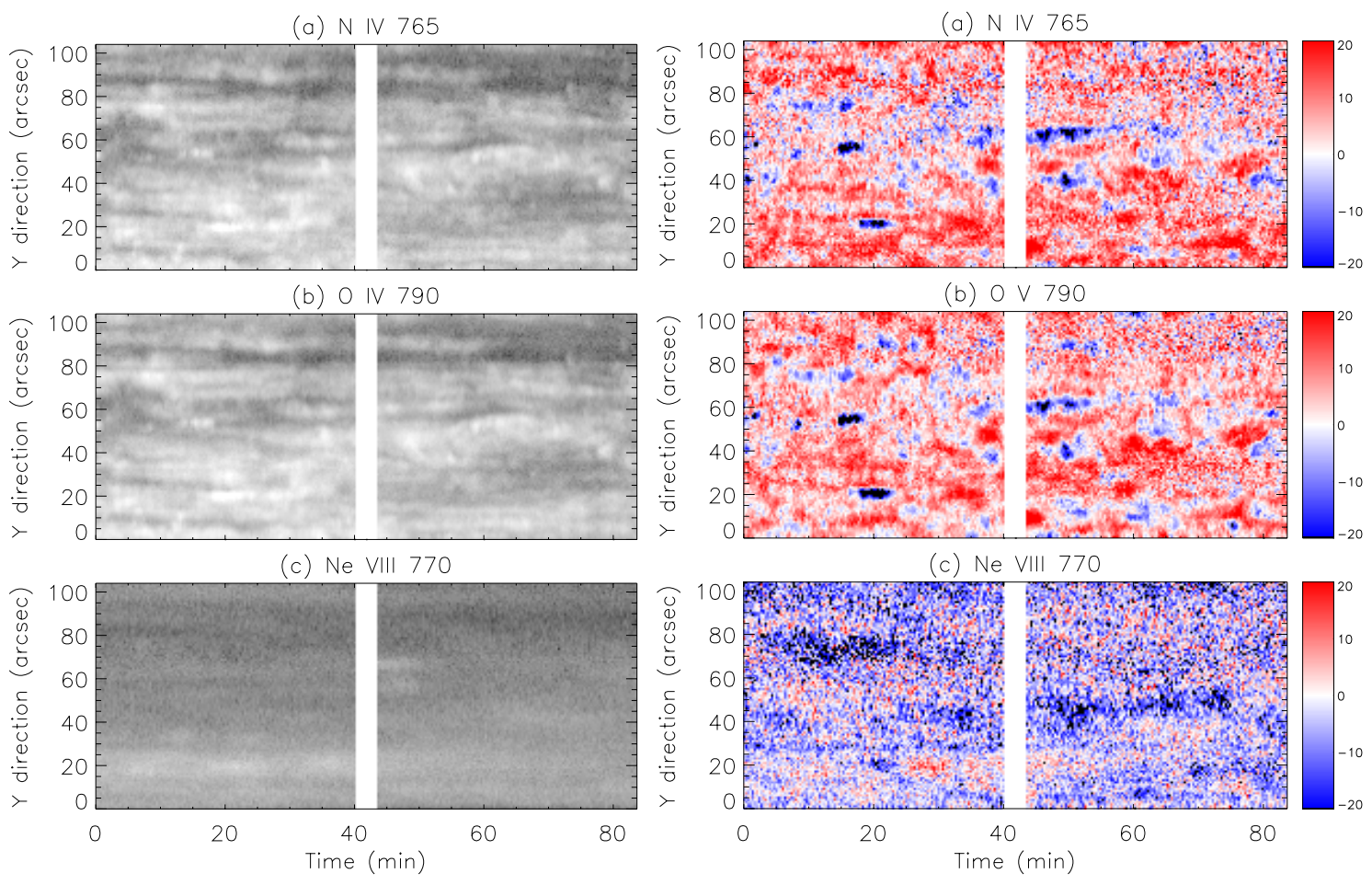

Figure 5.25: Images in intensity (logarithmic scale) and line shift (right frames) in the spectral window from $760 \AA$ to $795 \AA$. (a) N IV (765 ^); (b) O IV (790 ̊) and (c) Ne VIII (770 ̊). An exposure time of $20 \mathrm{~s}$ has been applied.

From Figure 5.27, this event had a visible spatial size of about $8^{\prime \prime}$ along the slit direction and lasted 3 raster steps, which equals about $4.5^{\prime \prime}$ along the raster direction, if the effect of the solar rotation is included. The lifetime can thus be determined to be longer than 7.5 min, according to the total time that the SUMER slit has scanned across the bright area.

Furthermore, it can be seen that the N v lines was largely enhanced and broadened, and attained non-Gaussian profiles (see Figure 5.27). The central intensities increased to about 40 times the normal network value. The full width at half maximum (FWHM) reached about $1.5 \AA\left(360 \mathrm{~km} \mathrm{~s}^{-1}\right)$. The continua and $\mathrm{C}$ I lines were enhanced by a factor of about 10 through the entire spectral window. The $\mathrm{Mg}$ X line can be no longer identified in the spectra. This means that this line has no prominent enhancement in emission during the event. Moreover, the integrated intensity of the two $\mathrm{N} V$ lines increased by a factor of about 300. They can be separated roughly by two Gaussians, whose central positions deviated by about $\pm 95 \mathrm{~km} \mathrm{~s}^{-1}$ from the normal central position, respectively.

We suggest that this transient event (network flare) has properties similar to an explosive event, but with a larger size and a longer lifetime as compared with a typical explosive event (lifetime of about $1 \mathrm{~min}$ and size of about $2^{\prime \prime}$ ). It is interesting that this event has also a large enhancement in the continuum and $\mathrm{C}$ I emission, which is not obvious and hardly observed in normal explosive events. 


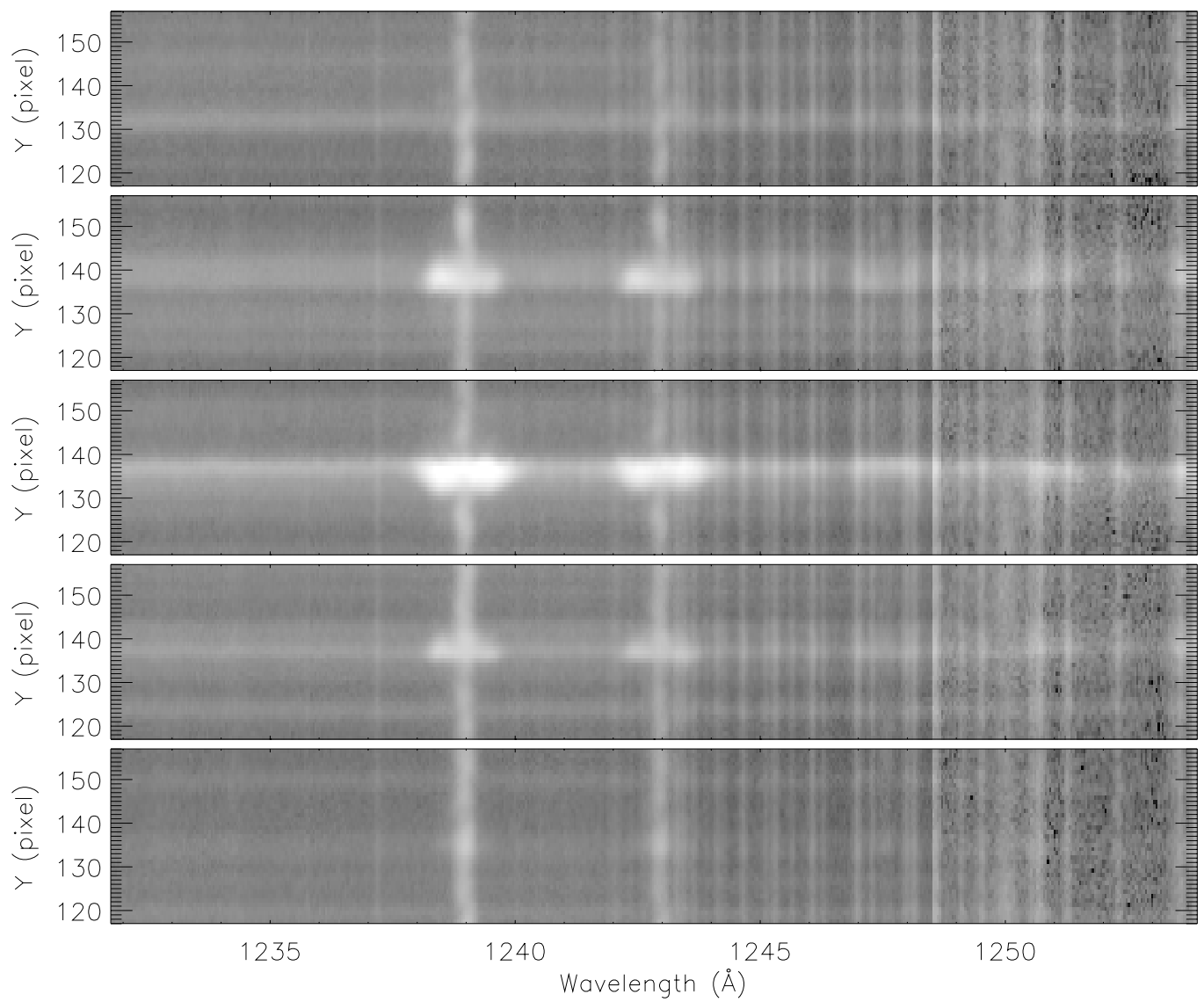

Figure 5.26: SUMER spectral window from $1234 \AA$ to $1254 \AA$ which includes the $C$ I lines, two $N \mathrm{~V}$ lines and a $\mathrm{Mg} X$ line (in 2nd order). Only part of the detector readout is shown around the transient event located from spatial pixels 130 to 140. The 4 rasters were taken sequentially with an interval in time of $150 \mathrm{~s}$ and a raster step of $1.13^{\prime \prime}$. Note the dramatic broadening of the $N \mathrm{~V}$ lines and the enhancement of emission along the whole spectral window.

\subsection{Summary and discussion}

In this chapter, the general morphological properties of the equatorial coronal holes have been discussed. By combining the observations of SUMER, EIT, MDI and NSO/KP, various structures in the coronal holes and the relationships between the line parameters (intensity, Doppler shift and width) as well as the underlying magnetic field have been studied. At the same time, a comparison of the $\mathrm{CH}$ and QS regions has also been made. We summarize some results and conclude as follows:

- The bases of equatorial coronal holes as seen in chromospheric lines have generally similar properties as normal QS regions. Small bright points with a size of about $2^{\prime \prime}-10^{\prime \prime}$ are the predominant features in the network as well as in cell interiors. With the increase of the temperature, these features become less pronounced. An obvious difference has been found in the shape of the $\mathrm{H} \mathrm{I} \mathrm{L} \beta$ line, which has much asymmetric (towards 


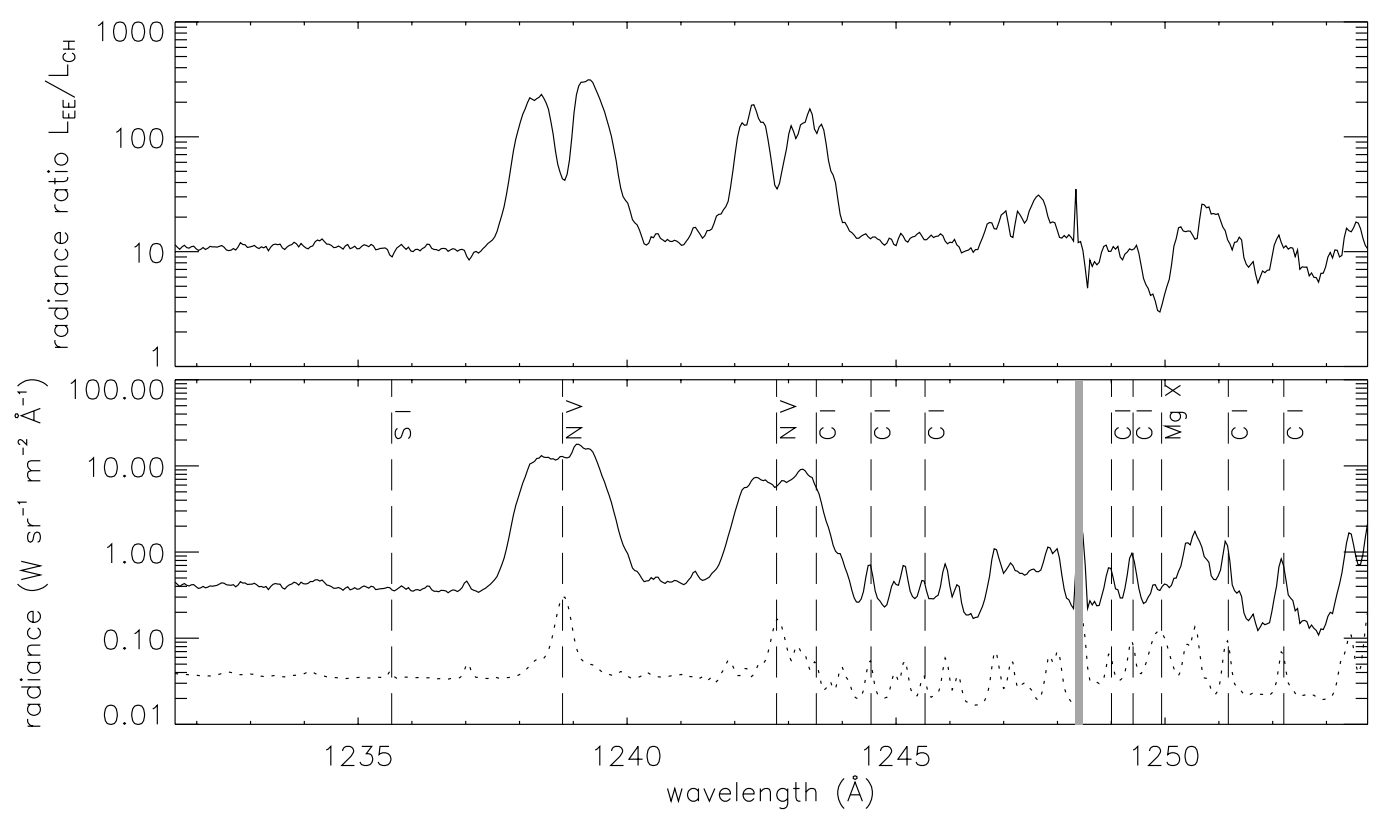

Figure 5.27: Spectral window from $1234 \AA$ to $1254 \AA$ which includes the $C$ I lines, two $N V$ lines and a $M g X$ line (in 2nd order) showing a transient event. The solid line represents the spectrum of the transient event, while the dotted line for averaged profile obtained from the normal network in the $\mathrm{CH}$ observed on 12 March, 1999.

the blue side) profiles in the coronal hole.

- The network width as seen in all transition region lines appears to be broader in $\mathrm{CHs}$ than in the QS. Loop-like structures are the most prominent features in the transition region, in both $\mathrm{CH}$ and QS regions. They have visible widths of about $10^{\prime \prime}$ and lengths of about $10^{\prime \prime}-30^{\prime \prime}$, and can be seen more clearly in lines emitted by Li-like ions and also in the H I, He I and C III lines. In coronal holes, we found that many of such structures seem to have one footpoint rooted in the intranetwork and to extend into the cell interiors. Some of them appear as star-shape clusters.

- In Dopplergrams of the O VI line, there are also fine structures with apparent blue shifts, although they are on average associated with red shifted. Many structures appear as loop-like and spatially coincide with the locations of the loop-like structures seen in the intensity image, while some appear as blue points, which have less extension and are located at the same footpoints of the loop-like structures seen in the intensity image. The blue structures usually have a broader line width. They seem to represent plasma above the concentration of a large unipolar magnetic field, without obvious bipolar magnetic features nearby. Moreover, the locations with blue shifts seen in the O VI line are usually also blue shifted if seen in the $\mathrm{L} \beta$ line. For the $\mathrm{C}$ II line, blue shifts appear seldom in Dopplergrams, but if visible, they are of smaller magnitude, the corresponding regions of smaller spatial size.

- Some coronal holes have a very different appearance as seen in the Ne VIII line in comparison with as observed in the Fe XII and Fe XV EIT channels. We suggest that this 
difference may have two reason: First, the average magnetic flux density (signed) is small in such coronal holes. Second, there are many small bipolar magnetic structures present there, leading to formation of small bright structures that dominate the emission observed in lines with lower formation temperatures, but such bright structures eventually disappear with the increase of temperature, and thus are not observed in the coronal channels of EIT.

- The Doppler shifts derived from the Ne VIII and Mg X lines are on average blue in the coronal holes. Red shifts are present only in bright structures, with mixed-polarity magnetic structures underlying. Conversely, the larger blue shifts are mainly associated with the dark regions, where the strong magnetic flux of a single polarity is concentrated. On the other hand, the Dopplergram of the $\mathrm{Mg} \mathrm{X}$ line is more homogeneous in the hole area. It seems that this is in agreement with a geometry of the hole consisting of coronal funnels, namely, the corona must be more uniform at a higher altitude. The results are in agreement with the notion that the fast solar wind is initially accelerated in such open magnetic funnels. The measured outflow velocity in the central region of the network is comparable with the values predicted by the models.

The results given here are obtained mainly from our qualitative studies. Some of them will be confirmed again by a more quantitative analysis, which will be described in details in the next chapter. 



\section{Chapter 6}

\section{Statistical and Quantitative Analysis}

\subsection{Introduction}

It has long been known that the coronal holes are the source of the fast solar wind (see, e.g., Krieger et al., 1973; Nolte et al., 1976)). Since the Skylab time, various efforts have been made to investigate the signatures of the fast solar wind, by both morphological and statistical studies and by comparison of the coronal holes and the quiet Sun. One of the most interesting topics is the velocity field in the coronal hole. As was discussed in Chapter 2, early work has been done by measuring the Doppler shifts of several coronal lines such as the Mg IX, Mg X and Si XI lines. During the SOHO mission, one gained advantage from the observations with the high spectral resolution of the SUMER. Such work has been done by many authors for both the quiet Sun and polar coronal holes (PCHs) (see,e.g., Brekke et al., 1997; Hassler et al., 1999; Peter and Judge, 1999; Wilhelm et al., 2000). In polar coronal holes, the average Doppler shift of the Ne VIII (770 $\AA$ ) line and the relationship between Doppler shift and chromospheric network has been studied by Hassler et al. (1999). They found observational evidence that the largest outflow velocity is closely associated with the underlying chromospheric network, whereby they used the Si II (1533 A) brightenings in the radiance image as an indication of the chromospheric magnetic network. An extended work, done by Stucki et al. (2000a), who did a statistical analysis, showed a positive correlation between the blue shift of the Ne VIII line and the intensity of the N IV line (765 $\AA$ ) (used also as an indicator of the network) in the polar coronal hole, and thus corroborated the results of Hassler et al. (1999).

Another interesting observational result is that the largest blue shifts of the Ne VIII line coincide spatially with very dark regions in the intensity image of the coronal hole when seen in the same line (Wilhelm et al., 2000). This has been confirmed in Section 5.6 by inspection of the Dopplergram of the Ne VIII line together with the magnetogram observed by NSO/KP, in which it was found that bright points seen in this line have usually smaller blue shifts.

By systematically analyzing various lines obtained by SUMER in the quiet Sun, the temperature dependence of the average Doppler shift and line width has been studied by some authors (Chae et al., 1998c a; Peter and Judge, 1999; Teriaca et al., 1999) However, a systematic investigation in the equatorial coronal hole by using SUMER data has not yet been carried out. A similar analysis of the SUMER data in ECHs is of great interest, because the viewing conditions near the disk center are suitable for an investigation of the Doppler shift, in particular for the upper transition region and coronal lines. Moreover, the magnetic field measurements are more reliable near the disk center than on the disk far from the center. We are thus able to make an analysis, combining SUMER spectroscopic data with magnetic field maps, as presented before in Chapter 5. Such a study can give 
us a clearer physical picture of the plasma conditions and flow pattern prevailing at the coronal hole base.

The chapter is organized as follows: we first give a brief description of the selection and analysis of data in the next section; The statistical study of the line parameters are presented in Section 3; In Section 4 we discuss the average properties of line parameters in both $\mathrm{CH}$ and QS regions; A temperature-dependence of the average line parameters will be summarized in Section 5; Then, a relationship between deduced line parameters of the coronal line of $\mathrm{Mg} \mathrm{X}$ and the underlying magnetic field will be investigated in Section 6; Finally, the summary and discussion are given in Section 7.

\subsection{Data selection and analysis}

In the following section we will discuss the average properties deduced from line parameters in coronal holes, i.e., the average intensity, Doppler shift and line width. The data are selected from 5 coronal holes and a QS region. Among them, $\mathrm{CH} 1$ and $\mathrm{CH} 2$ were observed during the solar minimum in 1996, while $\mathrm{CH} 6, \mathrm{CH} 7$ and $\mathrm{CH} 13$ were observed during the solar maximum in 1999. The instrumental parameters used by SUMER during these observations are given in Table 4.1. Since CH6 and QS1 were observed with both "raster scan" and "reference spectrum" modes and included a large number of lines emitted from different layers of the solar atmosphere, they will be used for a statistical study and comparison of their different properties between the $\mathrm{CH}$ and QS regions. For $\mathrm{CH} 1$, $\mathrm{CH} 2$ and $\mathrm{CH}$ 7, only the coronal line of $\mathrm{Mg} \mathrm{X}$ is analyzed to investigate the relationship between the hole corona and the underlying magnetic field.

The general description of the method of data analysis has been given in Chapter 4 . Here we give some additional explanations about the data reduction. There are three methods to deduce an average line parameters: one is simply to bin the individual profile over an observed hole area that we are interested in, and to fit afterward the averaged line profile; a second method is to deduce line parameters pixel by pixel, then calculate the average values; or one can first normalize the individual profile and then get an averaged profile for a further fitting to deduce the Doppler shift and line width. The reason to apply the last two methods is to avoid the intense profiles dominating the weak ones. This may occur if we study the difference between different structures with high contrast of the intensity, for example, a network and cell region or a bright point and dark region. In fact, previous studies (see, e.g., Brynildsen et al., 1998; Stucki et al., 2000a) have shown the correlation between the observed line shift and intensity. A discussion of this question can be found in previous papers (Chae et al., $1998 \mathrm{c}$; Teriaca et al., 1999$)$. In the quiet Sun, Teriaca et al. (1999) found no relevant difference between the first and second method. In our study, we also make such a comparison using the data observed on November 8, 1999 in $\mathrm{CH} 13$. The slit 4 was used. Three lines (O I at $1027 \AA, \mathrm{C}$ II at $1036 \AA$ and O VI at $1032 \AA$ ) with high signal-to-noise ratios have been analyzed. Between the two methods, a maximal difference of $0.05 \mathrm{px}\left(0.6 \mathrm{~km} \mathrm{~s}^{-1}\right)$ is found for the line shift, and $0.02 \mathrm{px}(0.3$ 
$\mathrm{km} \mathrm{s}^{-1}$ ) for the Doppler width, smaller than the uncertainty resulting from other analyzing procedures. Hence, we confirm the result obtained by Teriaca et al. (1999) that there is no essential deviation between the first two methods. According to these studies, we adopt the first method to deduce the line parameters for studying the average properties of the coronal hole in Section 6.4

\subsection{Statistical studies of line parameters}

In this section, statistical methods are applied to study the distribution of the line parameters, the relationship between the line intensity, Doppler shift and line width, as well as the difference between the $\mathrm{CH}$ and QS regions. By using the continuum as an indicator of the chromospheric network, the relationship between line parameters and the network is also discussed. The most data were obtained in March 1999 (CH6 and QS1), in which a large number of data samples were obtained.

\subsubsection{Distribution of line intensity, Doppler shift and width}

Histograms of the continuum, integrated line intensity, Doppler shift and line width are plotted in Figures 6.1, 6.2, 6.3, 6.4 and 6.5 for both $\mathrm{CH}$ and QS regions. Among them, all Doppler shifts are related to their average value for each region, and the line width is defined as $v_{1 / e}=c \Delta \lambda_{D} / \lambda$ (see Eq 3.29), from which the instrumental broadening has not been subtracted.

\section{Distribution of the intensity}

The distribution of the intensity has been investigated in equatorial coronal holes by several authors (see, e.g., Huber et al., 1974, Lemaire et al., 1999), as well as in polar coronal holes (see, e.g., Dammasch et al., 1999a; Stucki et al., 2000b, 2002). From the histogram of the intensity obtained in the QS, a low-intensity peak and a high-intensity tail, a pronounced characteristic related to the network and cell, can always be found for the chromospheric and transition region lines. By comparison of the $\mathrm{CH}$ and QS region, previous observations revealed that such a distribution is also present in the $\mathrm{CH}$ spectrum, but more sharply peaked in transition region lines (Stucki et al., 2002). In addition, the distribution of the intensity, if shown on a logarithmic-linear scale, was found to have a Gaussian shape in both regions (Dammasch et al., 1999a; Wilhelm et al., 1998a; Griffiths et al., 1999).

In this study, we start with describing such an intensity distribution for the chromospheric continuum. Figure 6.1 shows the intensity distribution of the continuum in three ranges of the wavelength (around $1539 \AA, 1235 \AA$ and $912 \AA$ ), which should be statistically reliable because they are obtained by averaging over $25-45$ spectral pixels. From this figure, 


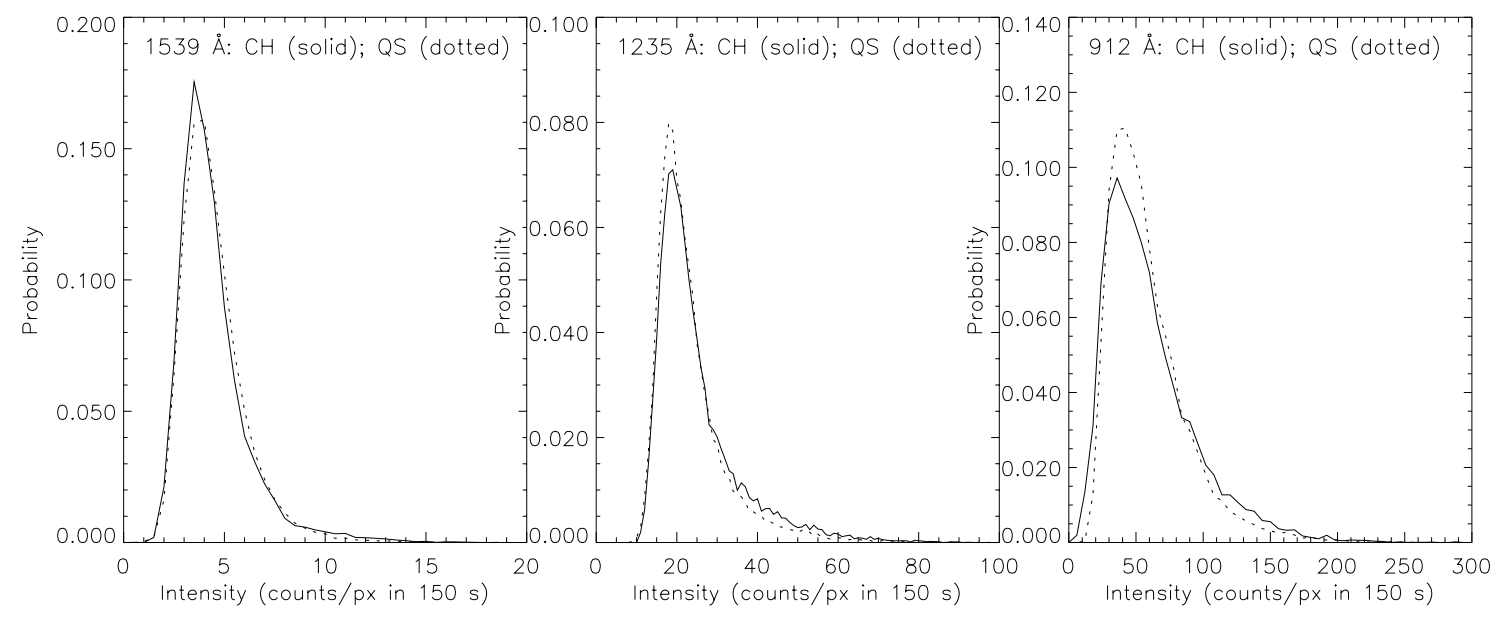

Figure 6.1: Histograms of chromospheric continuum at $1539 \AA, 1235 \AA$ and $933 / 912 \AA$, with the solid line for the $\mathrm{CH}$ and dotted one for the QS.

one can find that a prominent change of the profile obtained in different wavelengths. For the continuum at $1539 \AA$, the distribution is less different between the $\mathrm{CH}$ and QS region, except for a slightly higher peak for the $\mathrm{CH}$ data. This may indicate that the network and cell structure has only a slight difference between the two regions. On the other hand, a slightly higher peak and lower tail can be found for the QS data in the other two wavelengths. The latter situation can be also found in the Si II line (see Figure 6.2).

In Figure 6.2, the two chromospheric lines ( $\mathrm{Si}$ II and $\mathrm{He}$ I) reveal very different properties. The Si II line shows a similar property as the continuum at $1235 \AA$ and $912 \AA$. However, the $\mathrm{He} \mathrm{I}$ line observed in the $\mathrm{CH}$ region is more sharply peaked at the lower intensity, with a much smaller tail of the higher intensity, which indicates that the network becomes much weaker in the $\mathrm{CH}$ when seen in this line. This line behaves indeed like the transition region lines of $\mathrm{N} \mathrm{V}$ and $\mathrm{O}$ VI, which will be discussed in the following.

Three transition region lines (C II, C IV and S VI) exhibit similar distributions of their intensities in each region (see Figures 6.3 and 6.4). The $\mathrm{CH}$ data have a lower intensity of the peak contributed by the cell region, but with a higher probability, while the tails contributed by the network show less difference. For the other two transition region lines ( $\mathrm{N} \mathrm{V}$ and $\mathrm{O} \mathrm{VI}$ ), the difference between the $\mathrm{CH}$ and QS regions becomes more apparent. Like the He I, they have a much sharper distribution of intensities in the $\mathrm{CH}$ region, and their tails are weaker and less extended towards the high intensity. Clearly, this reflects the reduced contrast of the intensity between the network and cell regions. Such a tendency becomes much clearer for the Ne VIII and Mg X lines, in which the weakening tails for both regions may indicate the eventual absence of the network structure (see Figure 6.5), as was discussed in Chapter 2. Moreover, the $\mathrm{CH}$ data reveal a much higher peak, but at much lower intensity. Thus the $\mathrm{CH}$ region appears much darker and more uniform than the QS region.

From the distribution of intensities, there is evidence that a slight difference between the $\mathrm{CH}$ and QS regions has already been present in the upper chromosphere. The apparent dif- 

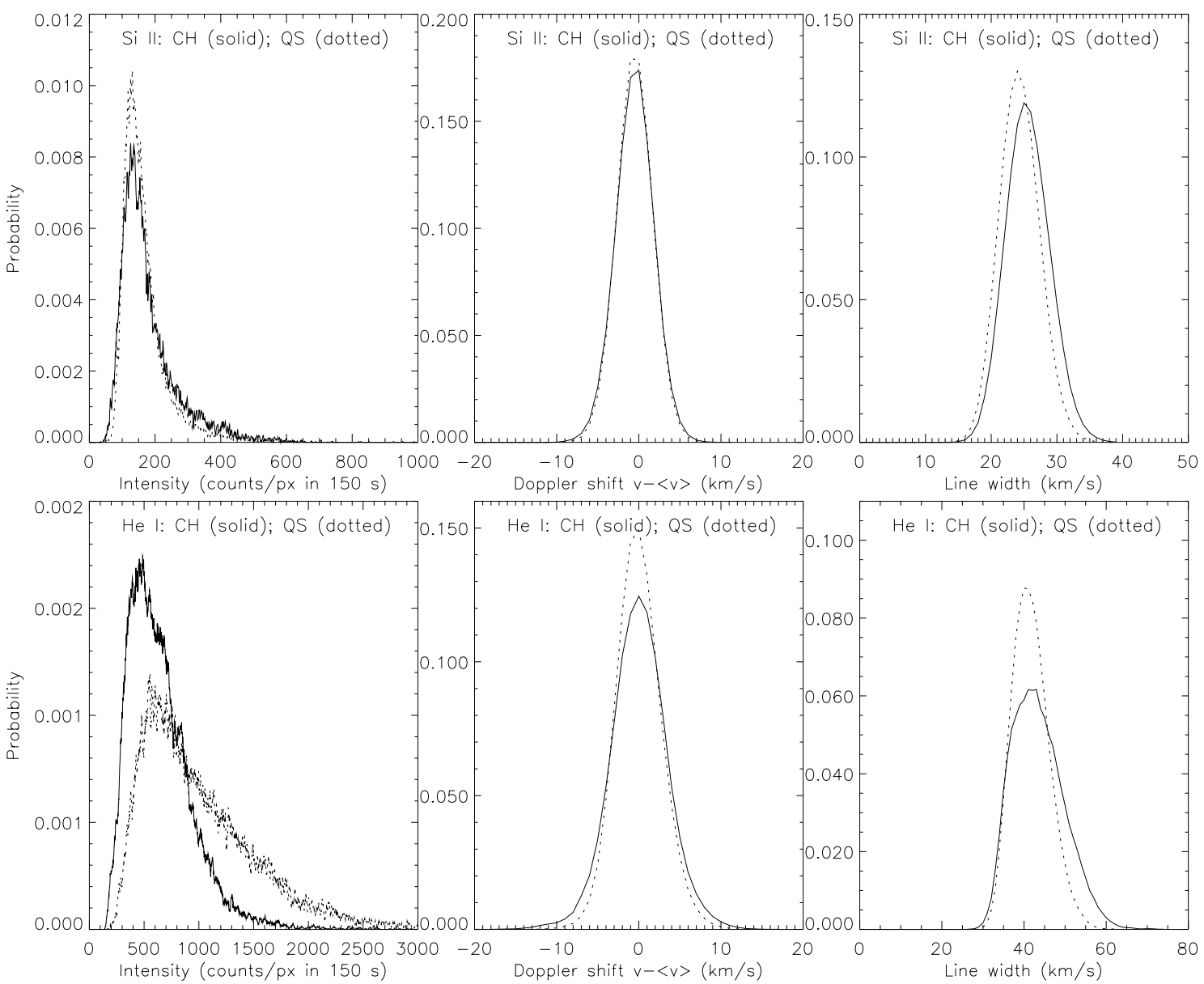

Figure 6.2: Histograms of integrated line intensity, Doppler shift and line width, with the solid line for the CH and dotted one for the QS. Upper panels: Si II; Lower panels: He I.

ference between the two regions, when seen in the He lines, has been found since a long time (see, e.g., Andretta and Jones, 1997). Previous SUMER observations also showed that the He I resonance line at $584 \AA$ is depleted in the polar coronal holes (Peter, 1999; Wilhelm et al. 2000). This helium line originates in plasma where helium is essentially partially ionized below the lower transition region, and thus it has a very complicated behaviour due to being sensitive to the line formation mechanism, particle diffusion and mass flow (see, e.g., Andretta and Jones, 1997; Fontenla et al., 2002). On the other hand, the obvious discrepancy being present in the $\mathrm{CH}$ and QS region, when seen in transition region lines emitted by Li-like ions of $\mathrm{N}^{4+}$ and $\mathrm{O}^{5+}$, may be directly determined by their wider formation temperatures, which means that they are emitted from a larger height range between the transition region and lower corona. The apparently high counts in the high-intensity tail are consistent with the morphological results that the loop-like structures seen in these lines are more extended into the cell interiors than those of other lines. 

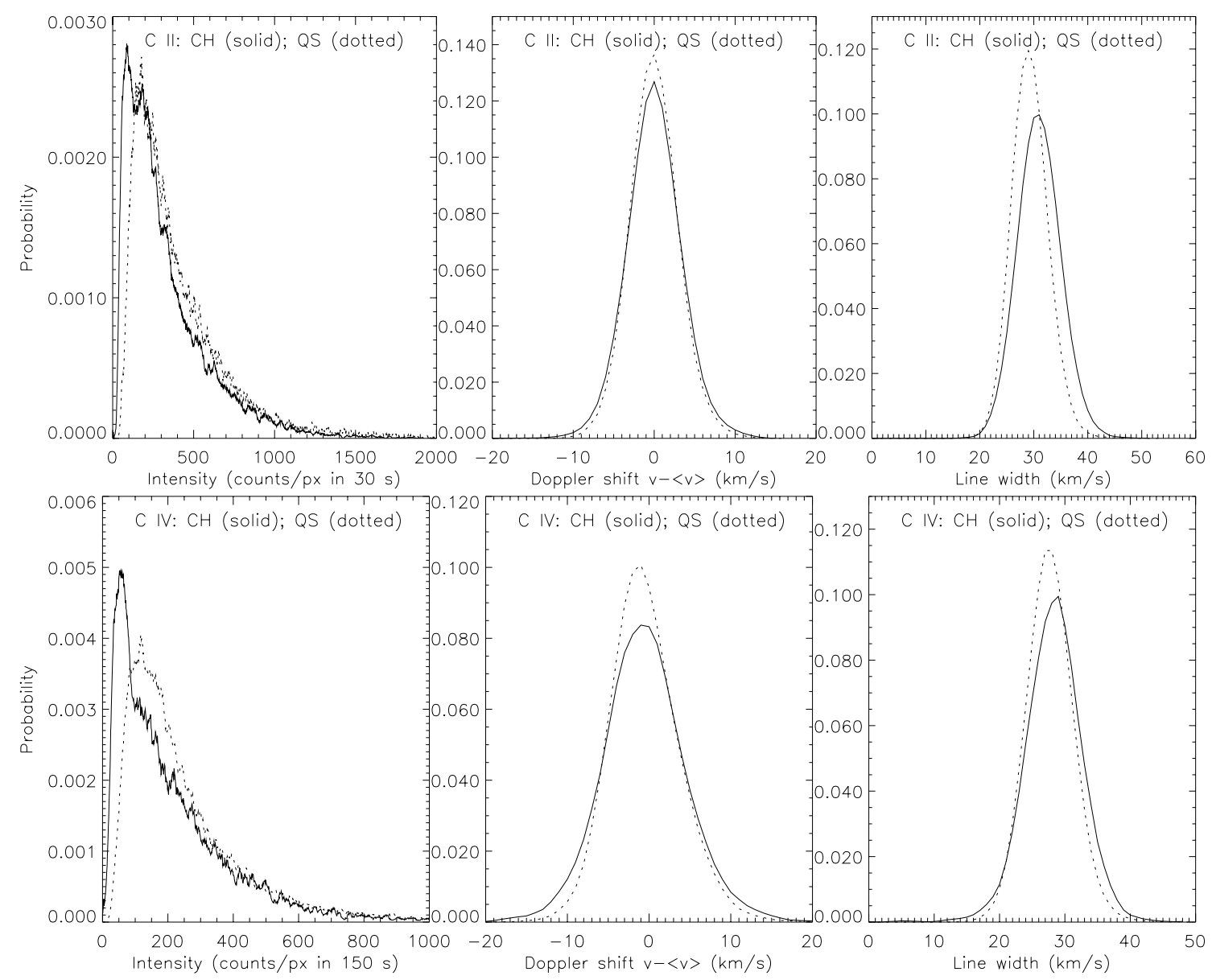

Figure 6.3: The same of Figure 6.2 but for $C_{I I}$ (upper) and $C_{I V}$ (lower).

\section{Distribution of the Doppler shift and line width}

At first sight the distribution of the Doppler shift and line width exhibits a nearly Gaussian shape for all lines studied here. This is in agreement with a previous result obtained in a polar coronal hole (Dammasch et al., 1999a).

Our statistical results show that the distribution of Doppler shifts has a temperaturedependent variation. For the chromospheric line of Si II, there is no obviously difference between the $\mathrm{CH}$ and QS regions. For the He I line, this distribution becomes wider in the $\mathrm{CH}$. The tendency is much more apparent for the O VI, Ne VIII and Mg X lines. In combination with the result that most lines formed in and above the transition region are on average bluer shifted in the $\mathrm{CH}$ than in the QS (this result will be discussed in the next section), this indicates that in the $\mathrm{CH}$ there are more areas with a blue shift of these lines, as it is expected when considering the origin of the fast solar wind.

A slightly larger line width in the $\mathrm{CH}$ than in the QS region can be found for all lines, which is generally in agreement with the result obtained in another equatorial coronal hole (CH5) (Lemaire et al., 1999). However, the wider line width shown in the chromospheric 

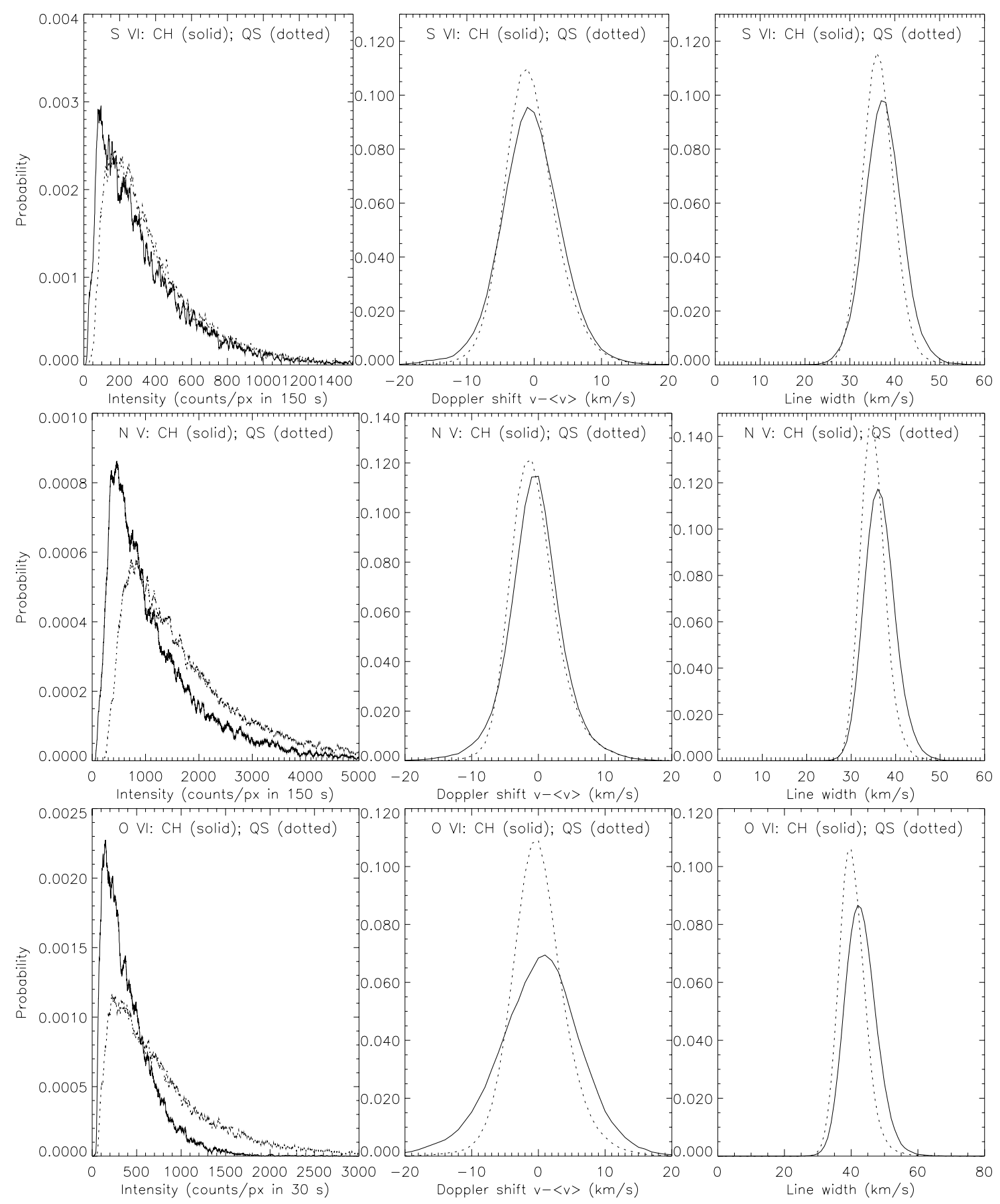

Figure 6.4: The same of Figure 6.2 but for $S_{V I}$ (upper), $N V$ (middle) and $O_{V I}$ (lower). 


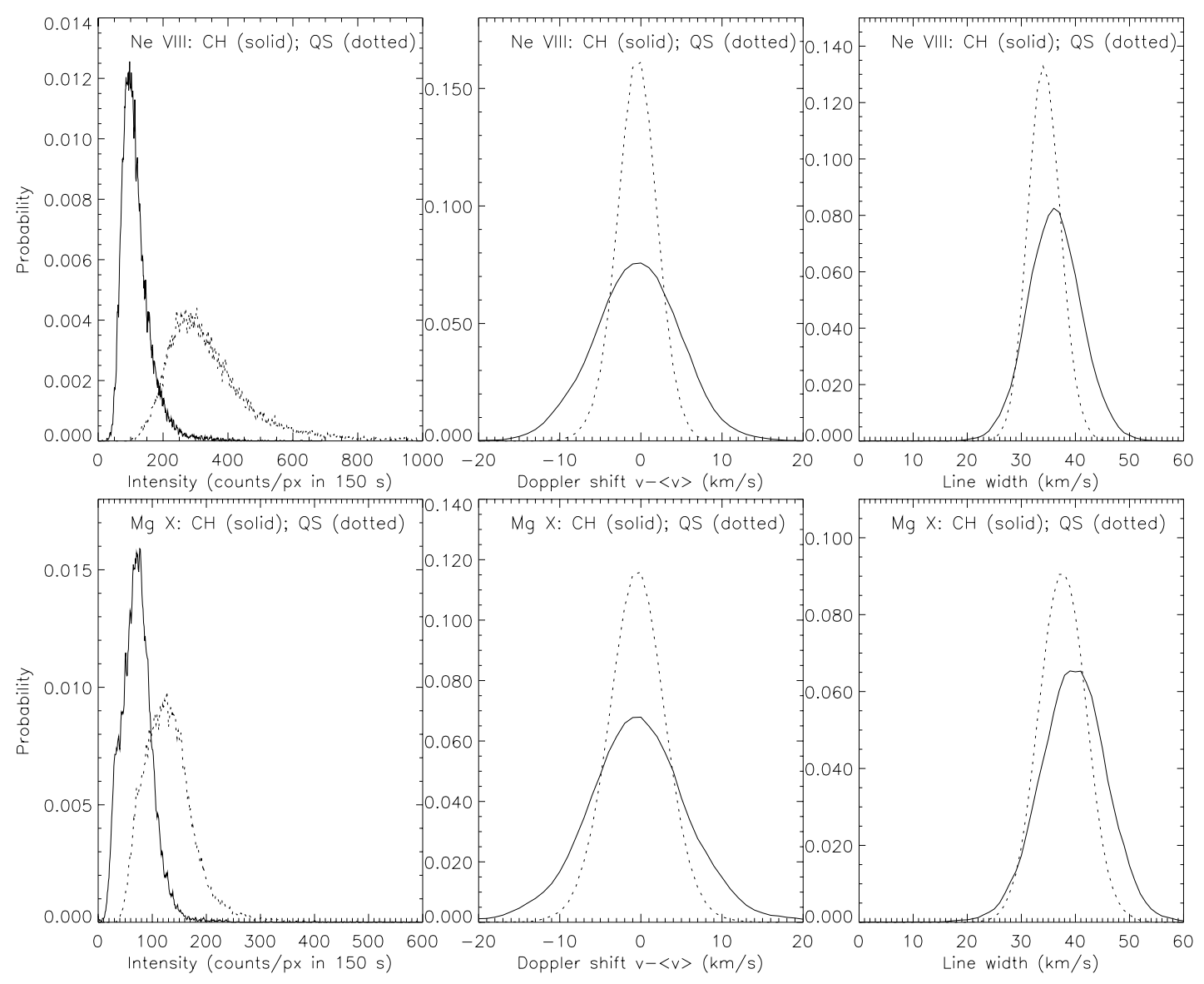

Figure 6.5: The same of Figure 6.2 but for Ne VIII (upper) and $\mathrm{Mg}$ X (lower).

lines should be interpreted with caution because they are less or more affected by opacity, which may also lead to broadening of the measured line width. A more quantitative analysis and discussion of this difference will be presented in the next section.

\subsubsection{Relationship between line parameters and the chromospheric network}

The relationship between the Doppler shift and the chromospheric network has been recently studied in polar coronal holes by some authors (Hassler et al. 1999; Stucki et al., 1999, 2000a). Their data showed a positive correlation between the Doppler shift of the $\mathrm{Ne}$ VIII line and the line intensity of chromospheric lines in polar coronal hole. In this study of equatorial holes, we show some more detailed statistical results concerning the relationship between the line parameters for a given line and the network structure seen in the chromospheric continuum.

As was discussed in Section 5.5, there exists a very close relationship between a brightening of the chromospheric emission and the magnetic network with a concentration of 

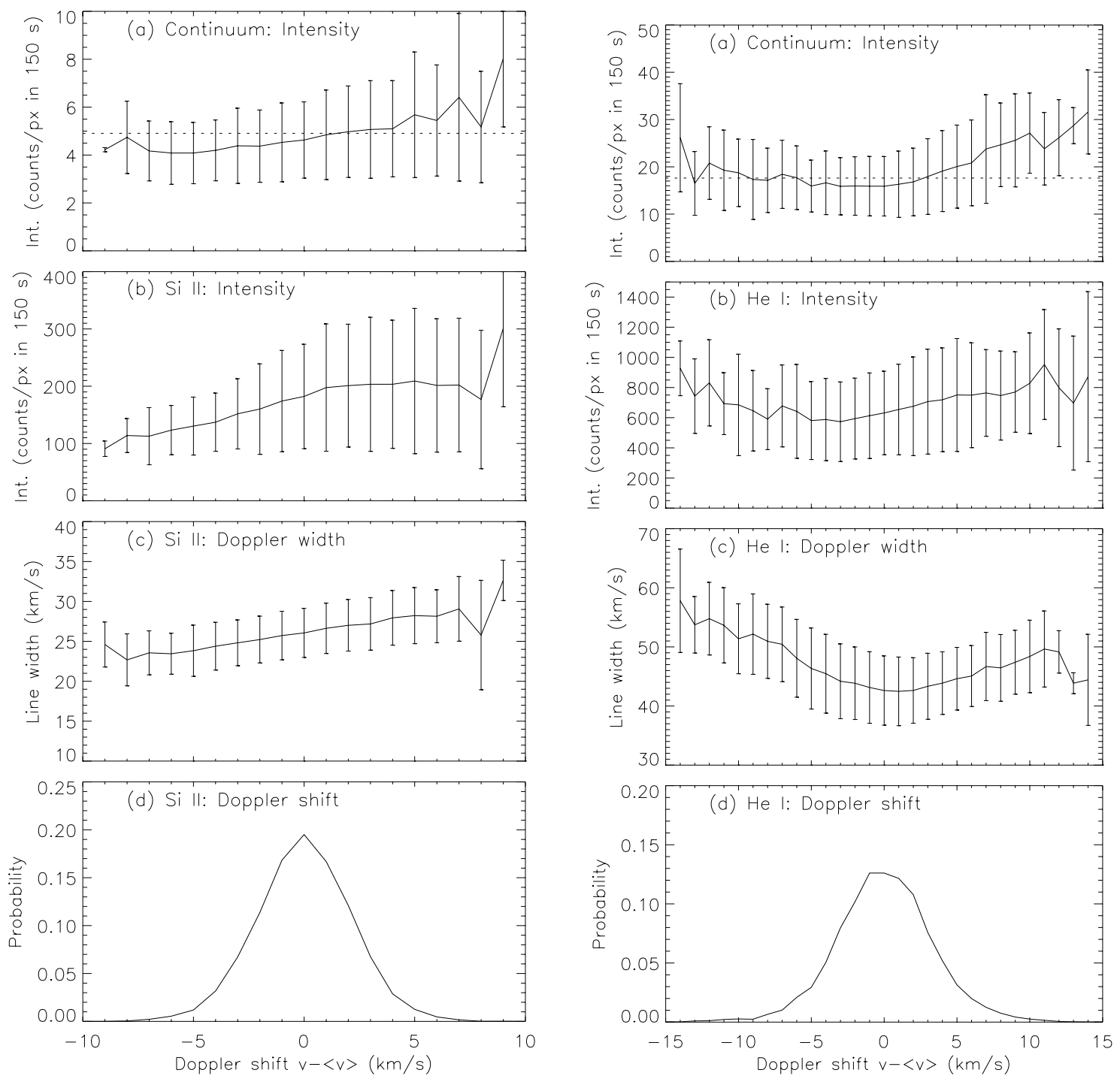

Figure 6.6: The relation between the chromospheric network and line parameters. The histogram of the Doppler shift with a bin-size of $1 \mathrm{~km} \mathrm{~s}^{-1}$ is shown in (d); The average Doppler width, integrated line intensity and continuum for each velocity interval are shown in (c), (b) and (a), respectively. The bar indicates the standard deviation of each parameter. The dotted line in (a) presents the threshold of the continuum intensity between the network and cell, set by the assumption that the network occupied 1/3 of the total area. Left panel: Si II; Right panel: He I.

the photospheric magnetic flux. Thus we can use an intensity map of the continuum as an indicator of the network in order to search for the relationship between line parameters and such network structures. The threshold of the intensity difference between the network and cells can be roughly determined by fitting the low-intensity peak in intensity histograms by a Gaussian profile (see discussion in Mariska, 1992). We find that the network area occupies around 1/3 of the total area determined by such a method using the continuum in different wavelengths. We thus adopt a threshold of the intensity determined 

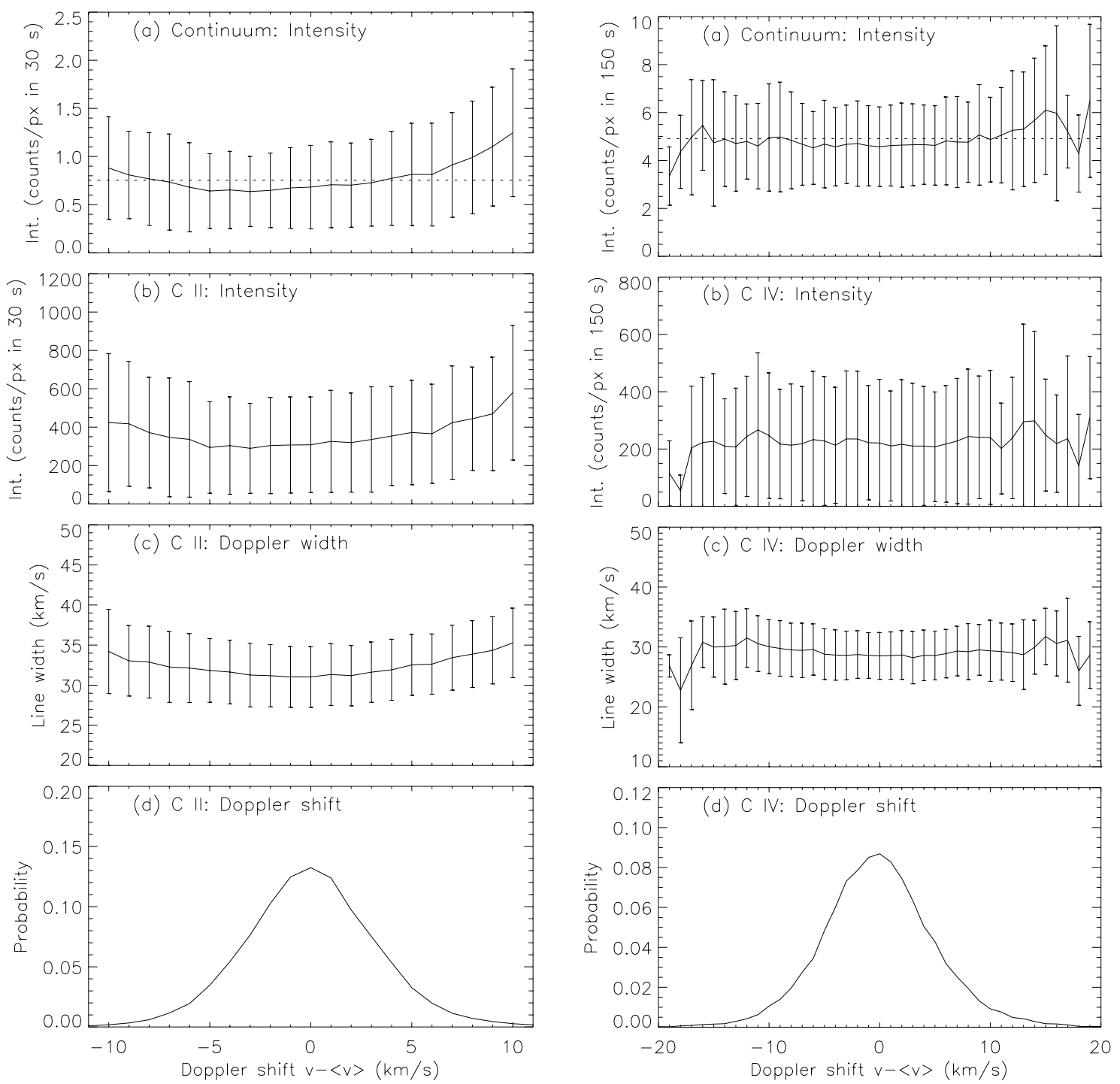

Figure 6.7: The same format as Figure 6.6 but for C II (left) and C IV (right).

by assuming that the network occupies $1 / 3$ of the total area.

The results are shown in Figures 6.6 to 6.9. The solid line in the bottom panel is the histogram of the Doppler shift with a speed interval of $1 \mathrm{~km} \mathrm{~s}^{-1}$, and the middle two panels indicate the average line width and the intensity of the same line, as calculated in each velocity interval. The same plot shown in the upper panel is for the continuum, which serves as an indicator of the chromospheric network. The dotted line represents the boundary between the network and cell region (above this value is network).

From the left column of Figure 6.6, one infers that the Doppler shift of the Si II line exhibits a very clear positive correlation with the line width. It is also clear that the relatively blue shifts correspond mainly to the lower intensities of this line and the continuum, i.e., from the cell region. The relatively red shifts relate to the higher intensities on average, 

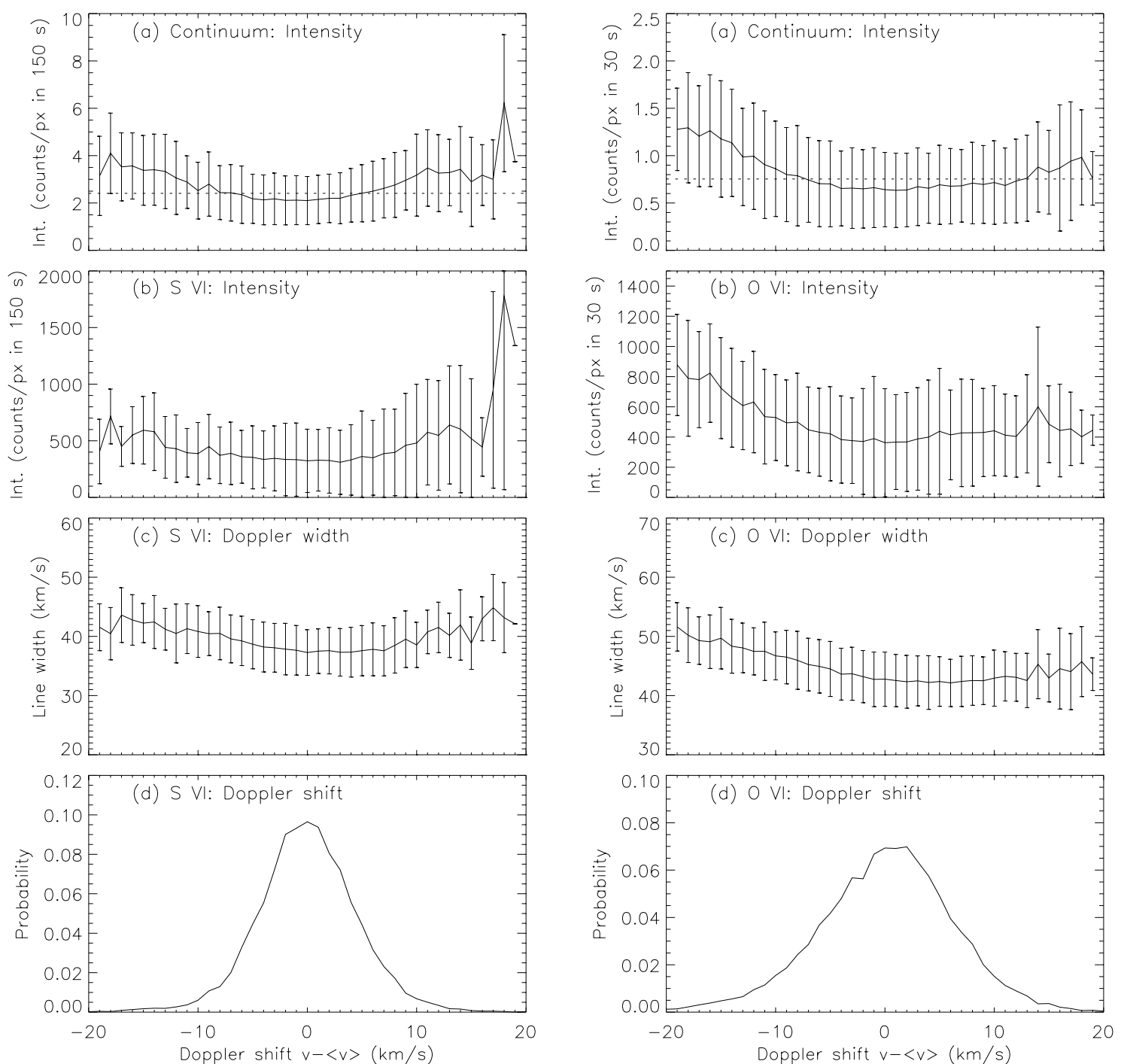

Figure 6.8: The same format as Figure 6.6 but for $S$ VI (left) and $O$ VI (right).

but with a large scatter for both intensities of the line and continuum. This implies that some of the red shifts are also produced in the cell region, although they mainly come from the network.

An apparent correlation between line parameters and the network can be found for the He I and transition region lines except for the C IV line. First, regions with an average Doppler shift (this average value $\langle v\rangle$ is set here as $0 \mathrm{~km} \mathrm{~s}^{-1}$ ) usually have the lowest average line width and intensity. They also correspond to the lowest average intensity of the continuum, which indicates that they are mainly contributed by the cell region. Second, the magnitude of the relative Doppler shift $(\mid v-\langle v>|)$ seems to have a positive correlation with the line width and intensity. A larger blue or red shift usually has a larger line width and intensity, and also corresponds to a larger intensity of continuum, which implies that they mainly occur in the network. 

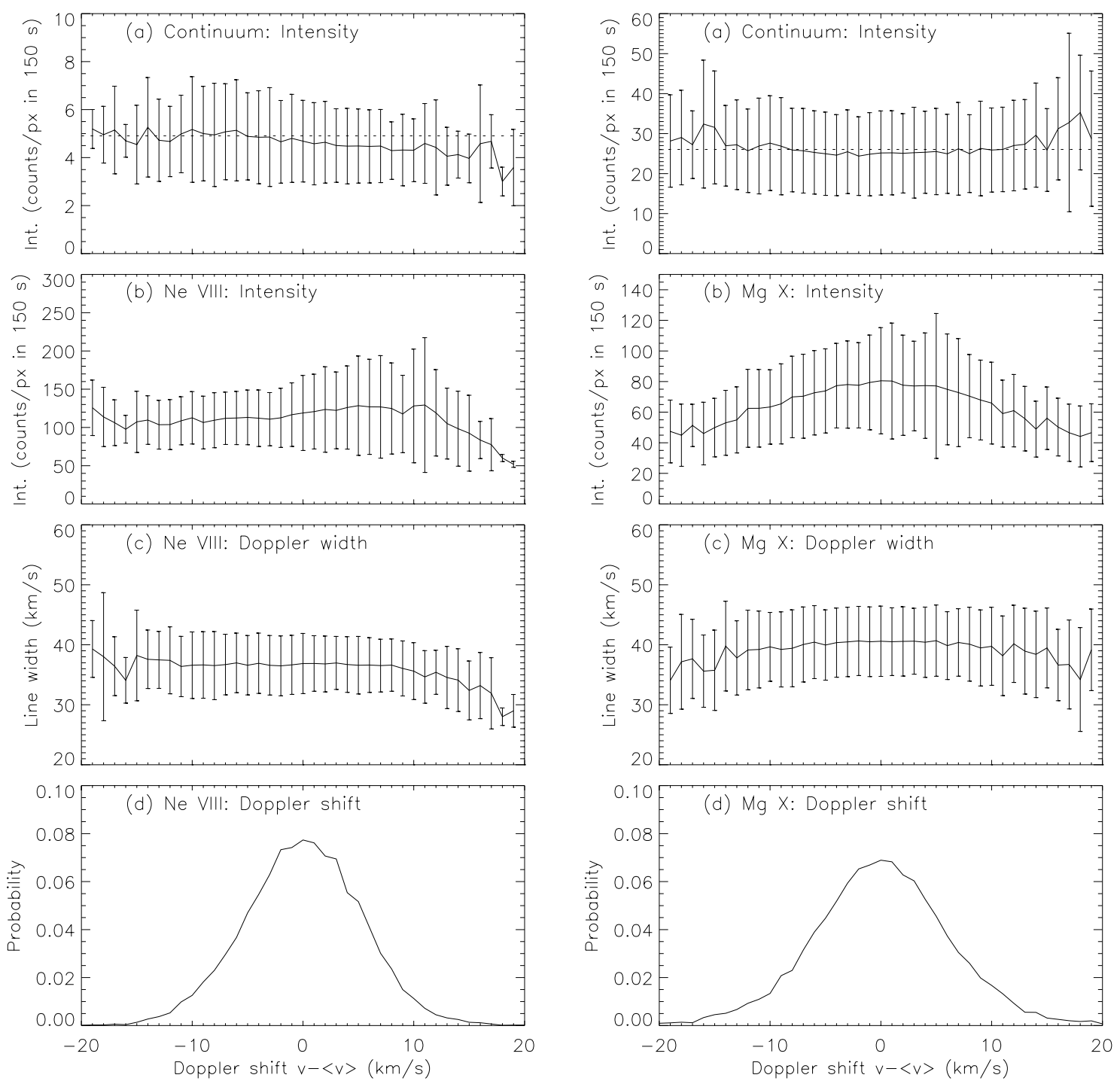

Figure 6.9: The same format as Figure 6.6 but for Ne VIII (left) and Mg X (right).

However, such a relationship is not clearly present for the C IV line, as was also found by Mullan and Waldron (1987) in a low-latitude coronal hole observed by SMM and recently confirmed by Peter (1999) in a polar coronal hole observed by SUMER. This is in contrast with the results observed in other regions on the Sun (Gebbie et al., 1981; Athay et al., 1983ab; Peter, 1999).

In the Ne VIII line, there exists a slightly positive correlation between the Doppler shift and intensity. A bluer shift tends to have a lower intensity and a redder one corresponds to a larger intensity. For the line width, the tendency reveals to be inverse. Compared with the continuum, the most counts with a larger blue shift have an averaged continuum intensity around the threshold defined as the limit between of network and cells. The result is consistent with that discussed in Section 5.6, in which it was found that larger Doppler 
shifts come mainly from the network with a concentration of an unipolar magnetic field, but with a larger areal extension as compared with the network area defined in chromospheric lines. This also confirms that such a tendency deduced from an equatorial coronal hole is similar to the one in polar coronal holes.

For the $\mathrm{Mg} \mathrm{X}$ line, the relationship between line parameters and the network seems to be different. Their line parameters show a very weak dependence on the network structure. Its average Doppler shift seems to correspond to the largest intensity and line width. It should be mentioned that this line is very weak in the coronal hole, and the large uncertainty of the deduced parameters for pixels with weak radiance may influence the distribution of the parameters. In spite of this, a very weak correlation between line parameters and the network indicates that the coronal hole seen in this line appears more uniform. This is consistent with the concept of an open funnel structure, with its area increasing with the altitude in the coronal hole.

\subsubsection{Relationship between Doppler shifts of different lines}

From the morphologic study in Chapter 5, it is found that the locations with blue shifts seen in the $\mathrm{O}$ VI line are usually blue shifted if seen in the $\mathrm{H} \mathrm{I} \mathrm{L} \beta$ and $\mathrm{C}$ II lines, but often appearing as small blue points with a smaller value of the blue shift and spatial size. These lines were simultaneously observed in the same spectral window. We use here a statistical method to look for the relationship between Doppler shifts of these lines. In Figure 6.10. the histogram of the Doppler shift (O VI) with a bin-size of $1 \mathrm{~km} \mathrm{~s}^{-1}$ is plotted in the bottom panel. The Doppler shifts of two other lines (H I L $\beta$ and C II), averaged in each velocity interval of the $\mathrm{O}$ VI line, are shown in the middle and upper panels. The bar indicates the standard deviation. The two datasets were obtained on March 11, 1999 in $\mathrm{CH} 6$ (left column) and on 8 November, 1999 in $\mathrm{CH} 13$ (right column).

In Figure 6.10, one can find a clear positive correlation between the Doppler shifts. Moreover, the corresponding magnitudes of the Doppler shifts vary with different lines formed at different layers, i.e., a larger value of the Doppler shift seen in the O VI line corresponds to a smaller one in the $\mathrm{C}$ II line and an even smaller one in the $\mathrm{H} \mathrm{I} \mathrm{L} \beta$ line. This statistical result nicely confirms our previous result obtained in the morphologic study.

If a Doppler shift is considered to be caused by a steady mass flow, the result also naturally agrees with a convection pattern obey the law of mass-flux conservation in a flux tube. This means that the flow velocity should be lower in the chromosphere due to the higher plasma density there than in the transition region. On the other hand, considering a partial mass flux taken away by the solar wind would also reduce the average red shift of a line, even though the average Doppler shift is absolutely red (Pneuman and Kopp, 1978). This may also be true for all transition region lines, as will be further discussed in the following section. 

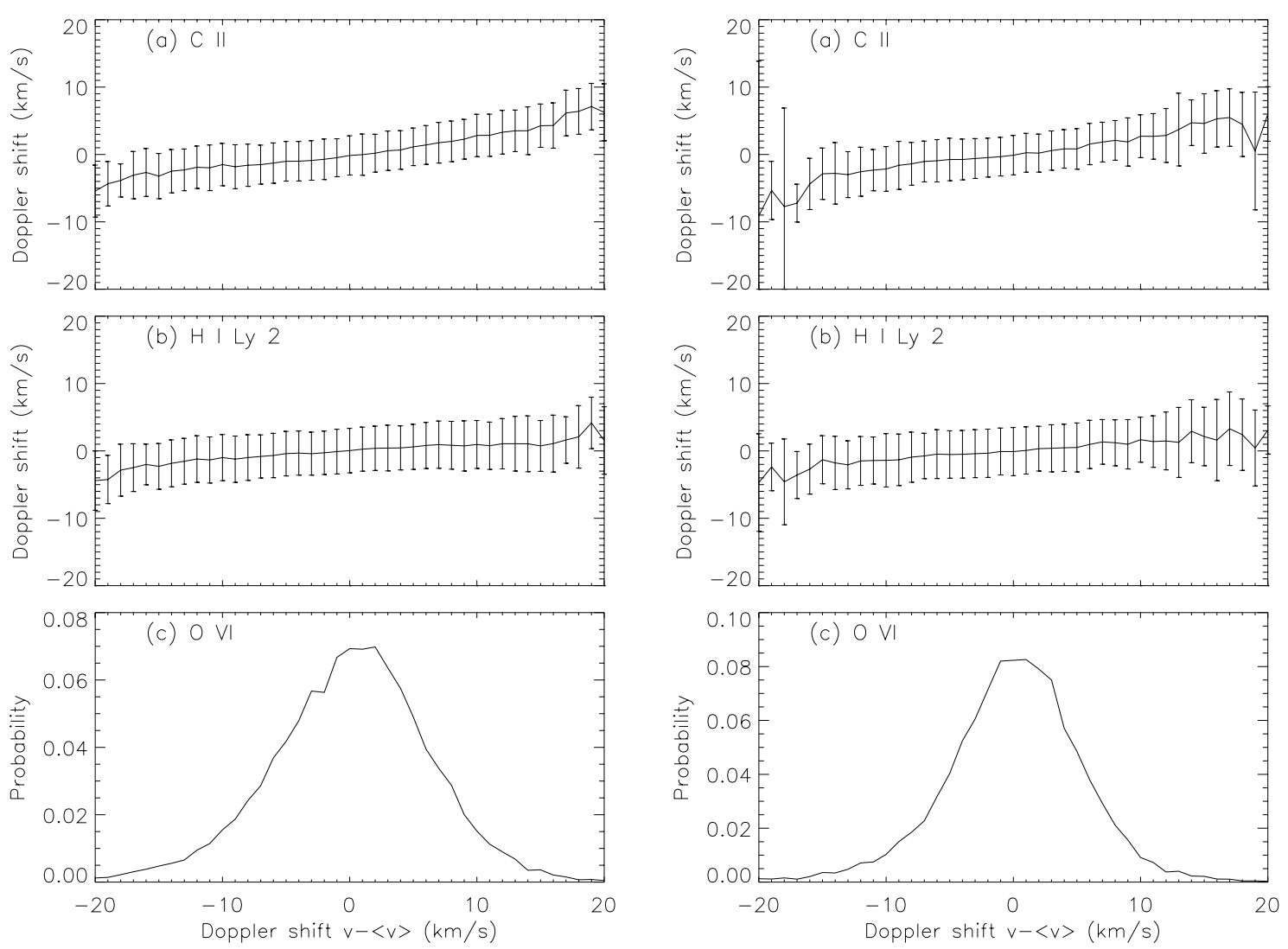

Figure 6.10: Relationship between Doppler shifts of different lines. The histogram of the Doppler shift (O VI) with a bin-size of $1 \mathrm{~km} \mathrm{~s}^{-1}$ is shown in (c); The Doppler shifts of other lines (H I L $\beta$ and C II) averaged in each velocity interval of the $O$ VI line are shown in (b) and (a), respectively. The bar indicates the standard deviation. Left: Data observed on March 11, 1999 in CH6; Right: Data observed on November 08, 1999 in CH[13.

\subsection{Average intensity, Doppler shift and line width}

An average intensity, Doppler shift and line width has been derived for various lines from the averaged spectrum for both QS and $\mathrm{CH}$ regions. Note that if spectral profiles of some lines are shown in the following, a radiometric calibration has been applied always in 1st order to correct the different spectral responsivity at different wavelengths and on different portions of the detector. This is different from the results listed in the tables, in which a line recorded in the 2 nd order of diffraction has been correspondingly calibrated in 2 nd order.

In this section the following notation will be used: Remark $(R)$ in the first column denotes the "reference spectrum" used by SUMER, otherwise a "raster scan" mode is used. If more than one line of the same ion is measured, a notation of "(1)", "(2)", ,..... is used to denote different wavelengths. The first and second letter of "AK, AB, BK, BB" before the pixel number in the second column represent detector $\mathrm{A} / \mathrm{B}$ and $\mathrm{KBr} / \mathrm{bare}$ portion of detector, respectively. In addition, all intensities listed in the tables are integrated line intensity. 
The errors given for Doppler shifts are calculated by the relation, $\sigma=\sqrt{\sigma_{f t}^{2}+\sigma_{r f}^{2}+\sigma_{g c}^{2}}$, where $\sigma_{f t}, \sigma_{r f}$ and $\sigma_{g c}$ denote the fitting error, the error of dispersion relation derived from the reference lines and the error of geometric calibration, respectively, as was discussed in Section 4.4.1.

In addition, the regions are indicated in the first column of each table. The detector and the line position in pixel address (in a reversed spectral coordinate, i.e., the pixel address begins at a shorter wavelength) are listed in the second column. It should be noted that the positive velocity represents the red shift and the negative one is for the blue shift, which we will always use in the following discussion.

\subsubsection{Lines with formation temperatures $T_{e}>0.5 \mathrm{MK}$}

\section{$\operatorname{Mg} X$ at $625 \AA$}
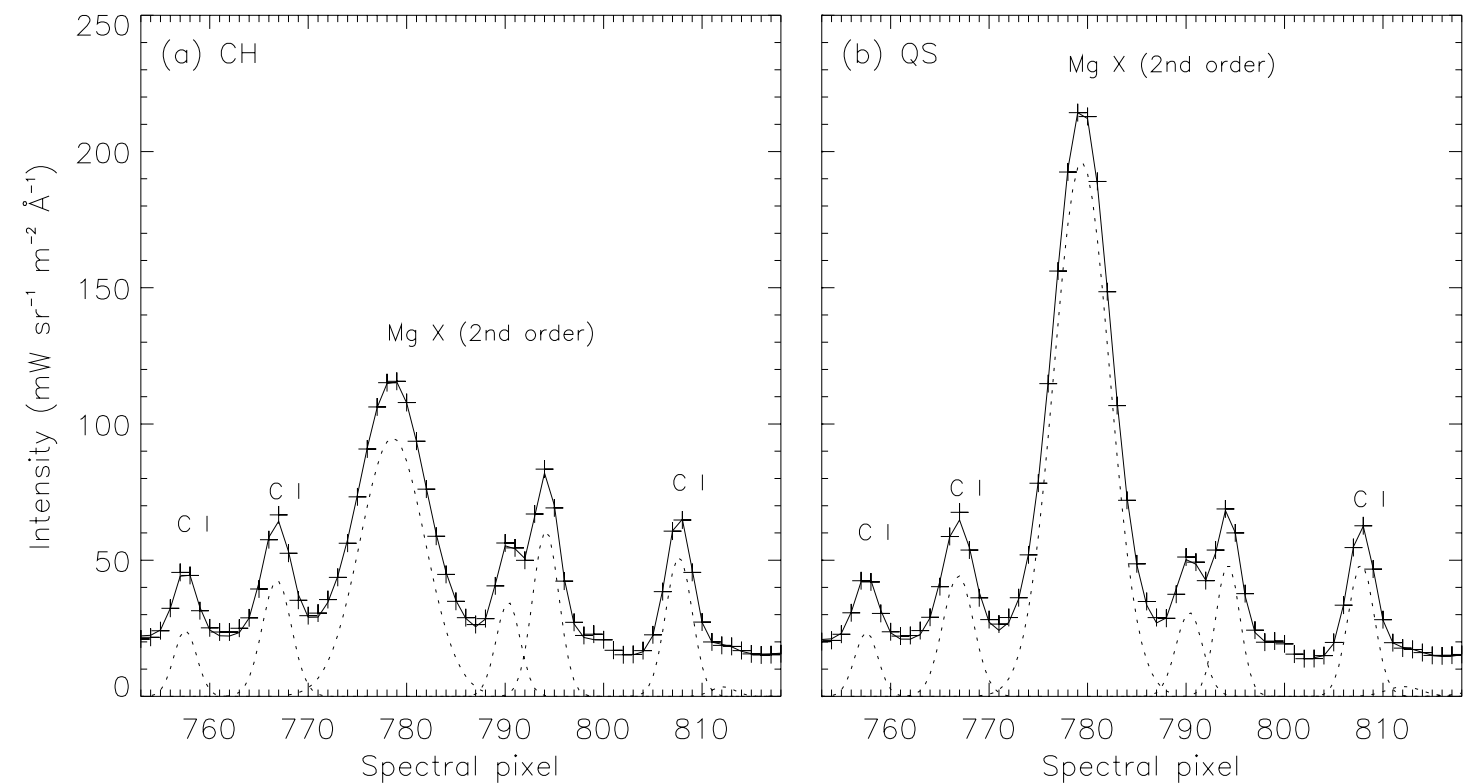

Figure 6.11: Line profiles of the $M g X$ line with its reference lines of $C$ I nearby. The "+" represents the data points from observations. Dotted lines are line profiles fitted with the Gaussian shape, while the solid lines represent the profiles obtained by background plus Gaussian fitting.

In the present data, the $\mathrm{Mg} \mathrm{X}$ line with a high formation temperature of $1.1 \mathrm{MK}$ is the only coronal line that can be used to reliably measure the absolute Doppler shift in coronal holes. This line is of particular interest for studying the coronal plasma, and can be detected with a good signal-to-noise ratio in second order on the bare portion of SUMER's detectors. On the $\mathrm{KBr}$ portion it is usually very weak or almost absent in coronal holes, and blended by two weak lines (Si II and P II?) (see discussion in Dammasch et al., 1999b). However, these first-order blends can be largely depressed in the bare portion that are used by us. In addition, the spectrum includes a series of $\mathrm{C} I$ lines nearby that can be used as 
Table 6.1: Derived line parameters for the $\mathrm{Mg} X$ line

\begin{tabular}{|c|c|c|c|c|c|}
\hline Region & Pixel & $\begin{array}{l}\text { Dispersion } \\
\text { (mÅ/pixel) }\end{array}$ & $\begin{array}{c}\text { Doppler shift } \\
\left(\mathrm{km} \mathrm{s}^{-1}\right)\end{array}$ & $\begin{array}{c}\text { Intensity } \\
\left(\mathrm{mW} \mathrm{sr}{ }^{-1} \mathrm{~m}^{-2}\right)\end{array}$ & $\begin{array}{l}\text { Non-thermal v. } \\
\left(\mathrm{km} \mathrm{s}^{-1}\right)\end{array}$ \\
\hline $\mathrm{CH} 1(\mathrm{R})$ & BB 207 & 42.75 & $-9.4 \pm 2.0$ & $2.8 \pm 0.3$ & $22.5 \pm 2.1$ \\
\hline $\mathrm{CH} 2(\mathrm{R})$ & BB 211 & 42.73 & $-2.9 \pm 2.4$ & $2.8 \pm 0.3$ & $15.1 \pm 2.3$ \\
\hline CHE(R) & AB 218 & 42.94 & $-4.5 \pm 1.9$ & $45.6 \pm 1.6$ & $32.9 \pm 0.8$ \\
\hline $\mathrm{CH} \overline{6}(\mathrm{R})$ & AK 513 & 43.42 & $-8.0 \pm 1.9$ & $39.1 \pm 3.7$ & $30.5 \pm 2.3$ \\
\hline $\mathrm{CH}$ & AB 779 & 43.33 & $-5.0 \pm 1.6$ & $41.4 \pm 1.0$ & $38.1 \pm 0.7$ \\
\hline $\mathrm{CH} \overline{\overline{6}}(\mathrm{R})$ & AB 808 & 43.12 & $-6.3 \pm 1.6$ & $49.5 \pm 1.7$ & $30.1 \pm 1.0$ \\
\hline QS1 & AB 219 & 42.96 & $1.3 \pm 1.6$ & $71.1 \pm 1.2$ & $27.9 \pm 0.4$ \\
\hline QSi1 $(\mathrm{R})$ & AK 514 & 43.38 & $-1.3 \pm 1.7$ & $70.0 \pm 5.1$ & $24.9 \pm 1.4$ \\
\hline QS1) & AB 779 & 43.23 & $2.9 \pm 1.5$ & $73.8 \pm 0.7$ & $29.4 \pm 0.3$ \\
\hline QS1 $1(\mathrm{R})$ & AB 808 & 43.14 & $0.1 \pm 1.5$ & $75.4 \pm 1.2$ & $23.9 \pm 0.4$ \\
\hline $\mathrm{CH}$ & AB 774 & 43.27 & $-7.1 \pm 1.9$ & $25.6 \pm 1.0$ & $29.9 \pm 1.1$ \\
\hline $\mathrm{CH} 13(\mathrm{R})$ & AB 221 & 43.01 & $-7.5 \pm 1.0$ & $8.9 \pm 0.7$ & $32.9 \pm 1.8$ \\
\hline $\mathrm{CH} \overline{\overline{13}}(\mathrm{R})$ & AB 811 & 43.16 & $-12.2 \pm 0.9$ & $9.6 \pm 0.7$ & $34.9 \pm 1.8$ \\
\hline
\end{tabular}

reference lines to determine the line shift (see Figure 6.11). In this study, four of them ( $1249.004 \AA, 1249.405 \AA, 1251.176 \AA$ and $1252.208 \AA$ ) are used to calculate the dispersion relation and the relative shift of the $\mathrm{Mg}$ X line. With them the dispersion relation can be calculated with a very small uncertainty if the signature is strong enough. This is indeed valid for the data obtained during solar maximum. The averaged profiles obtained in $\mathrm{CH}$ and QS regions ( $\mathrm{CH} 6$ and QS1) together with the fitted profiles are shown in Figure 6.11. This line can be fitted nicely with Gaussian in both regions.

In addition, a modified rest wavelength (624.968 ^) determined by Peter and Judge (1999) and Dammasch et al. (1999b) has been applied. The previous versions of its rest wavelength were reported as $924.950 \AA$ (Kelly, 1987) and $924.943 \AA$ (Kaufman and Martin, 1991 ), which deviate, in terms of Doppler shift, from the new one by $-5.8 \mathrm{~km} \mathrm{~s}^{-1}$ and $-8.1 \mathrm{~km} \mathrm{~s}^{-1}$, respectively. The deduced line parameters for five coronal holes and one QS region are listed in Table 6.1.

The results listed in Table 6.1 show that the Doppler shifts in the $\mathrm{CH}$ regions range from $-2.9 \mathrm{~km} \mathrm{~s}^{-1}$ to $-12.2 \mathrm{~km} \mathrm{~s}^{-1}$, while in the QS region it has a small blue or red shift. A discrepancy of up to $4.5 \mathrm{~km} \mathrm{~s}^{-1}$ can be found in the same hole measured by different observations. Such a discrepancy may be caused by instrumental errors, arising possibly from the determination of the line position and local geometric distortions of the spectrum, which have not been sufficiently corrected by calibration, or/and by solar effects (position- and time-dependence of the coronal properties). From our data obtained in $\mathrm{CH} 6$ and QS1, we find that this discrepancy is reduced to be about $2 \mathrm{~km} \mathrm{~s}^{-1}$ if the Doppler shift is calculated relative to the QS region for each observation at the same position on the detector. Therefore, instrumental effects may likely influence the uncertainty of the 
measured absolute Doppler shift. A better way to compare the Doppler shift in different regions is to use data obtained under the same conditions during the observations, in order to reduce the systematic deviations. In this study, we did not evaluate such deviations between different data sets, but simply regarded them as a scatter of the results.

The integrated line intensity, which has been calibrated in 2 nd order, seems to be very different between different holes. A lowest value was measured at solar minimum in $\mathrm{CH} 1$ and $\mathrm{CH} 2$, while a large one in $\mathrm{CH} 6$. Compared with the QS region observed during the same period within several days, the $\mathrm{Mg} X$ line in $\mathrm{CH} 6$ is weaker by about $40 \%$.

The line width shown here has been transformed into a non-thermal velocity. A lower value can be found for the $\mathrm{CHs}$ observed at solar minimum in 1996. The $\mathrm{Mg} \mathrm{X}$ line in $\mathrm{CH} 6$ is on average larger by about $6 \mathrm{~km} \mathrm{~s}^{-1}$ compared with QS1.

\section{Ne VIII at $770 \AA$}
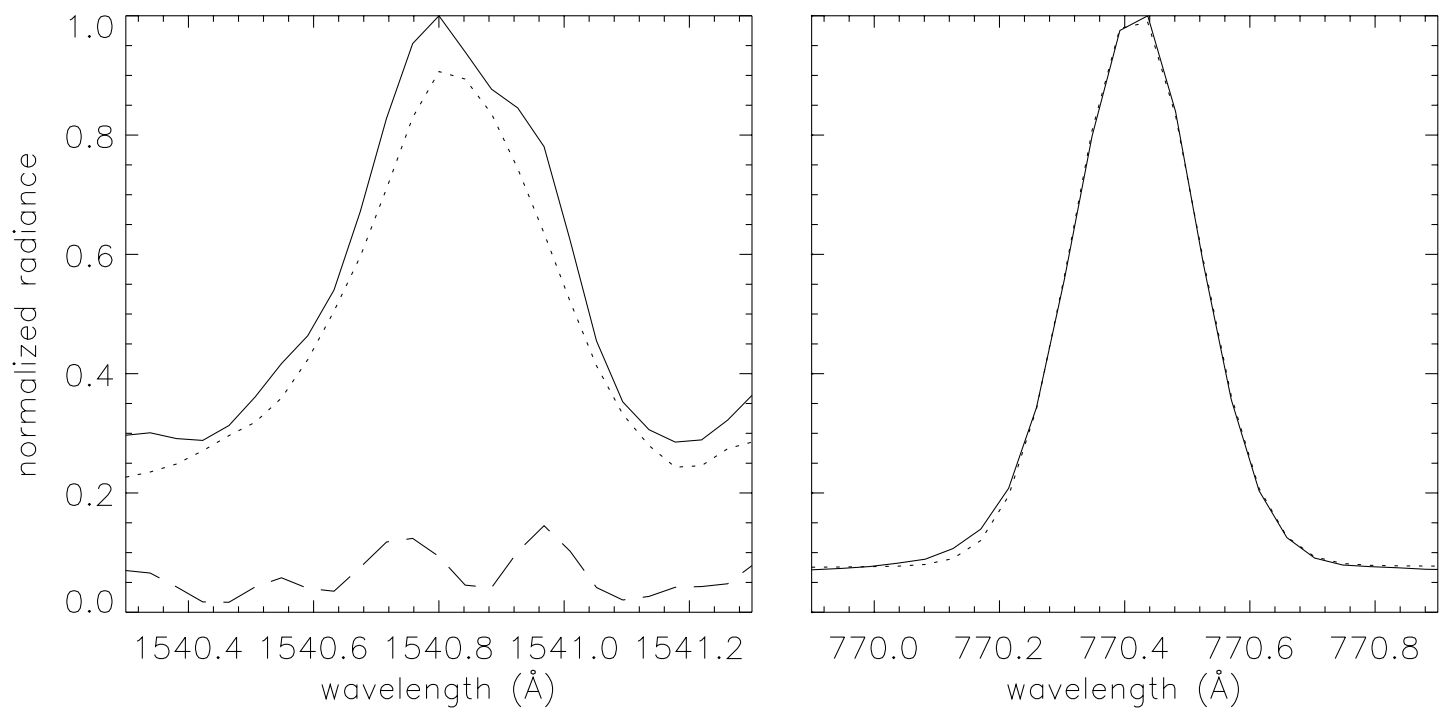

Figure 6.12: Left: Average line profile of the Ne VIII (770 A) line in the second order (solid line). This coronal hole profile was derived from observation in CH6 on March 11, 1999. It shows that there is a pronounced red shoulder which is also obtained by other investigators (Dammasch et al., 1999c; Wilhelm et al., 2002). The dashed line represents the synthetic profile of several blending Si I lines (from Kelly (1987)). The dotted line shows the profile of this line after the blended Si I lines are subtracted. It can then be seen that the red shoulder is almost removed. Right: Average line profile of the same line but observed in the first order (solid line). This coronal hole profile was derived from the observation in $\mathrm{CH} 33$ on October 19, 1996. It shows that it can be fitted by a single Gaussian very well (see dotted line) and obviously has no red shoulder. Other observations of this line in first order (detector B) in CHs yields a similar result.

The Ne VIII line has a formation temperature of $0.63 \mathrm{MK}$, and can be observed in first order on detector B and second order on detector A. In second order, the Doppler shift of this line can be determined with an absolute wavelength calibration by using the cooler $\mathrm{Si}$ 
II (1533.432 $\AA$ ) and C I 1542.1766 ̊ lines in the same spectral window as reference lines. However, this line is much weaker in coronal holes than in the quiet Sun, and blended by several Si I lines. The detailed average line profile of the Ne VIII (770 ̊) line in the 2nd order (solid line) is given in Figure 6.12. This coronal hole profile was derived from observations in $\mathrm{CH} 6$ on $11 \mathrm{March}, 1999$. It shows that there is a pronounced shoulder on its red wing, while its quiet-Sun profile has no shoulder. Similar profiles were also observed by other investigators in the polar coronal holes (Dammasch et al., 1999c; Wilhelm et al., 2000). Dammasch et al. (1999c) concluded that the shoulder could only partly originate from a blend of some $\mathrm{Si}$ I lines. Such a shoulder leads to a significant deviation of the $\mathrm{Ne}$ VIII line from a Gaussian shape, which leads to a further uncertainty in the determination of the Doppler shift of this line. It is necessary to distinguish if such a deviation is caused only by superposition of other lines or by an other unknown physical reason or by both effects.

Naturally, the red shoulder should not exist in profiles when observed in first order of diffraction if it is caused mainly by blends of others lines. We analyse such data obtained by SUMER in coronal holes with the first order of diffraction at wavelength $770 \AA$ using detector $\mathrm{B}$. The average line profile of this line is shown in Figure 6.12 (solid line), which was derived from observation in $\mathrm{CH} 3$ on 19 October, 1996. One can find that it can be fitted by a single Gaussian nicely (see dotted line) and obviously has no red shoulder. Other observations of this line in first order (detector B) in ECHs yields a similar result.

In fact, we can roughly eliminate the contribution of the blended components of other lines. The dashed line represents the synthetic profile of several blended Si I lines (from Kelly (1987)). The peak intensity of these blending lines can reach about $20 \%$ of the total one. The dotted line shows the profile of Ne VIII after the blending Si I lines are subtracted. It can then be seen that the red shoulder is almost removed. The central position of this line derived from the original profile is determined, by fitting Gaussian, to be red shifted by 0.15 pixels $\left(1.2 \mathrm{~km} \mathrm{~s}^{-1}\right)$ with respect to the profile obtained after subtracting the $\mathrm{Si}$ I blends. In the work of Hassler et al. (1999), they subtracted these blends using a firstorder High-Resolution Telescope and Spectrograph (HRTS) spectrum and found that this redshift was less than 0.03 pixels $\left(0.2 \mathrm{~km} \mathrm{~s}^{-1}\right)$. However, it seems that the peak intensity of the Ne VIII line obtained by them was stronger than the current one (see Figure 2B in their paper), so that the central position was less affected by the Si I blends.

Table 6.2 shows the deduced line parameters for coronal holes as well as in the QS region. The new rest wavelength (770.428 ̊) determined by Peter and Judge (1999) and Dammasch et al. (1999c) is used here. The wavelength reported by Kelly (1987) is $770.409 \AA$, a value of $-7.4 \mathrm{~km} \mathrm{~s}^{-1}$ shifted from the new one. Because it is obviously blended, the non-thermal velocity measured in the $\mathrm{KBr}$ portion of the detector is less reliable for the $\mathrm{CH} 6$,

The Doppler shift measured in this line is comparable with one obtained in the $\mathrm{Mg} \mathrm{X}$ line for each region. The intensity between the $\mathrm{CH} 6$ and QS1 is about 1/3 for the raster data. A larger non-thermal velocity by $7 \mathrm{~km} \mathrm{~s}^{-1}$ is found in $\mathrm{CH} 6$ than in QS1. In addition, the blue shift obtained in $\mathrm{CH} 13$ is consistent with the result discussed in Section 5.6, in which 
a large blue shift with more than $10 \mathrm{~km} \mathrm{~s}^{-1}$ was found above the network without bright points nearby.

Previous studies have also shown similar blue shifts for the Ne VIII line and coronal lines. For example, Wilhelm et al. (2002) obtained $5 \mathrm{~km} \mathrm{~s}^{-1}$ for Ne VIII and $10 \mathrm{~km} \mathrm{~s}^{-1}$ for $\mathrm{Mg}$ IX; Rottman et al. (1981, 1982) measured $12 \mathrm{~km} \mathrm{~s}^{-1}$ relative to outside the $\mathrm{CH}$ for the Mg X line (625 $\AA$ ); Cushman and Rense (1976) had results of $13 \mathrm{~km} \mathrm{~s}^{-1}$ for the Si XI line (303 $\AA), 12 \mathrm{~km} \mathrm{~s}^{-1}$ for the $\operatorname{Mg~X}$ line $(610 \AA)$, and $14 \mathrm{~km} \mathrm{~s}^{-1}$ for the $\operatorname{Mg} \mathrm{IX}(368 \AA)$, which are all relative to outside the $\mathrm{CH}$ region.

Table 6.2: Derived line parameters for the Ne VIII line

\begin{tabular}{l|c|c|c|c|c}
\hline Region & Pixel & $\begin{array}{c}\text { Dispersion } \\
(\mathrm{m} \AA / \text { pixel })\end{array}$ & $\begin{array}{c}\text { Doppler shift } \\
\left(\mathrm{km} \mathrm{s}^{-1}\right)\end{array}$ & $\begin{array}{c}\text { Intensity } \\
\left(\mathrm{mW} \mathrm{sr}^{-1} \mathrm{~m}^{-2}\right)\end{array}$ & $\begin{array}{c}\text { Non-thermal v. } \\
\left(\mathrm{km} \mathrm{s}^{-1}\right)\end{array}$ \\
\hline $\mathrm{CH} 6$ & AK 483 & 41.90 & $-5.5 \pm 2.1$ & $45.8 \pm 3.7$ & $30.6 \pm 1.3$ \\
$\mathrm{CH}$ (R) & AB 823 & 42.00 & $-5.4 \pm 1.7$ & $41.1 \pm 1.8$ & $32.6 \pm 0.8$ \\
Qs1 & AK 483 & 41.89 & $-1.5 \pm 1.9$ & $143.8 \pm 2.9$ & $25.3 \pm 0.4$ \\
QS11(R) & AB 821 & 42.01 & $-3.3 \pm 1.7$ & $90.8 \pm 0.8$ & $25.3 \pm 0.2$ \\
CH13(R) & AB 828 & 41.96 & $-11.8 \pm 1.6$ & $36.9 \pm 1.4$ & $34.2 \pm 1.0$ \\
\hline
\end{tabular}

\subsubsection{Transition region lines}

In Figure 6.13, three examples of averaged profiles are given for the $\mathrm{N} \mathrm{V}, \mathrm{S}$ VI and $\mathrm{O}$ VI lines in both $\mathrm{CH}$ and QS regions. Their profiles fitted by a single Gaussian are also plotted. From this figure, one may find a common feature that there is always a slight deviation between the observed profile and the fitted one at its low-intensity wings. This has led to an interpretation that the multi-component nature in the profiles of the transition region lines may reflect the complicated configuration of the network. As was discussed in Section 5.7.2, some authors (see, e.g., Peter, 2000, 2001, and references therein) treated the lines by fitting two Gaussians and found that the presence of a second broader spectral component of transition region lines is a general feature. This second component, with a broader and weaker profile, is suggested to originate from large loops in the quiet Sun or open funnels in the coronal hole, while the core (first component) stems from small cooler loops, and thus has a narrower line width and stronger intensity.

In this study of the coronal hole, we realize that the situation may be more complicated. From the morphological study in Sections 5.7.2 and 5.5, a coronal hole is often mixed with various structures, such as areas with an open magnetic field (funnels), bright points due to an interaction of the bipolar magnetic features and ambient unipolar ones, and small-scale loops associated with the magnetic activity along the network lanes. If a similar study should be carried out in the coronal hole, such different structures should treated 

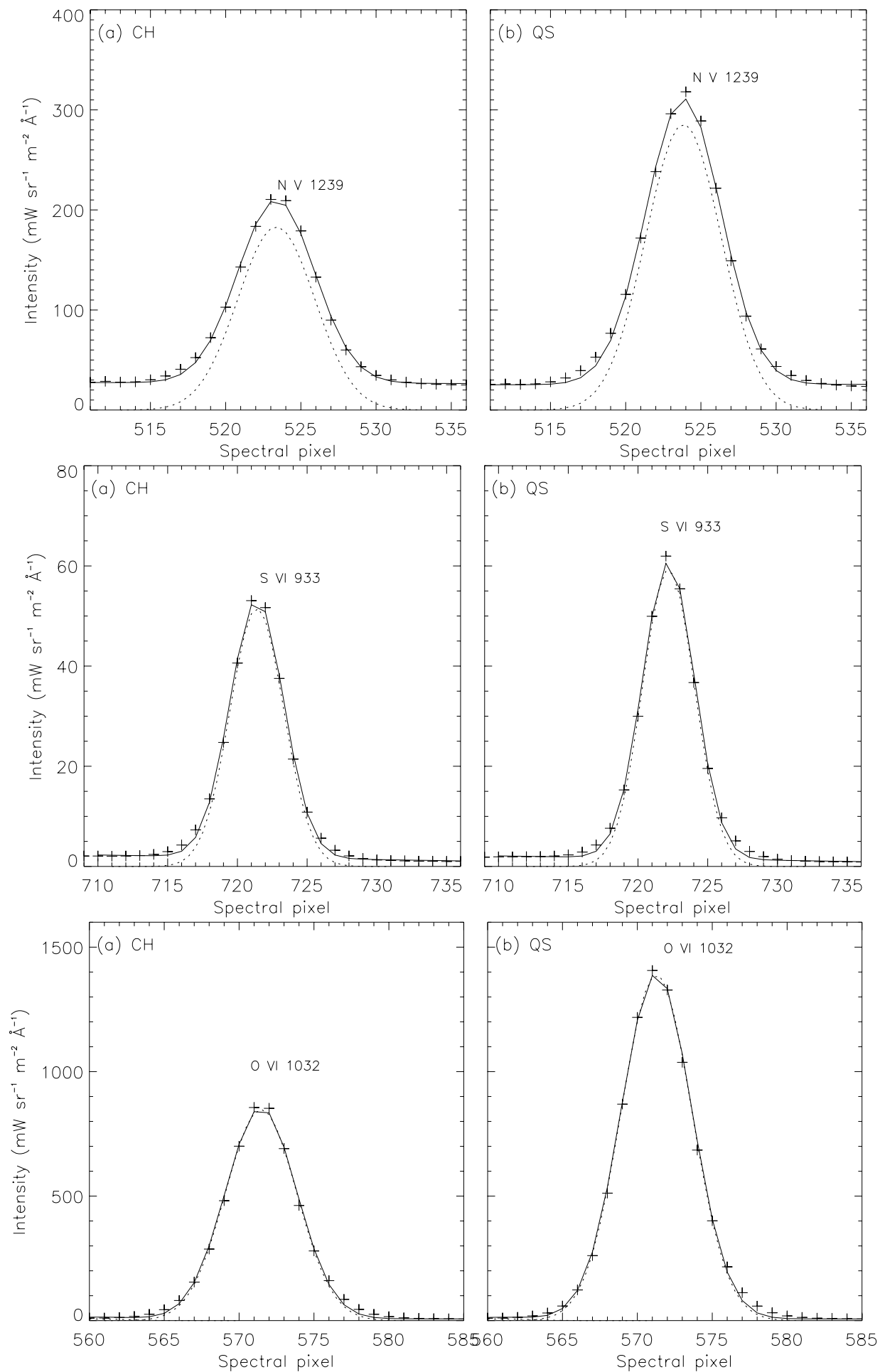

Figure 6.13: Line profiles of the $N$ V, S VI and $O V$ lines at $1239 \AA, 933 \AA$ and $1032 \AA$. The "+" represents the data points from observations. Dotted lines are line profiles fitted with the Gaussian shape, while the solid lines represent the profiles obtained by background plus Gaussian fitting. 
separately, which will increase the difficulties in the data analysis and physical interpretation. Hence, we have no attempt to do this in the following study, but only pay attention to the main features of the profiles.

\section{O VI at $1032 \AA$ and $1037 \AA$}

The O VI lines at 1031.9261 $\AA$ and 1037.6167 $\AA$ (Kaufman and Martin, 1989) have a very strong radiance with a high signal-to-noise ratio, and thus can be used for reliably deducing the line parameters with a high spatial resolution, as was discussed in Chapter 5. They are formed at $0.29 \mathrm{MK}$. The four O I lines $(1027.431 \AA, 1028.157 \AA, 1040.943 \AA$ and $1041.688 \AA$ ) in the same spectral window can be used as reference lines. Another $\mathrm{O}$ I line at 1039.230 was not used here, because it was located near the border of $\mathrm{KBr}$ and bare on SUMER's detector in some data sets.

From the results listed in Table 6.3, one can see that these two resonance lines have very different Doppler shifts in different coronal holes. A slight blue shift for O VI $1032 \AA$ and small red shift for O VI $1038 \AA$ are deduced for $\mathrm{CH} 13$. In other $\mathrm{CHs}$ and QS, these lines are red shifted. For each line, the shift is lower in the CHs than in the QS. However, the two lines have a different Doppler shift of about $2-3 \mathrm{~km} \mathrm{~s}^{-1}$. An even larger difference has been found by adopting the same rest wavelengths used here (Warren et al., 1997b a). The non-thermal velocity deduced for O VI $1032 \AA$ is systematically lower by $2-3 \mathrm{~km}$ $\mathrm{s}^{-1}$ than that of O VI $1038 \AA$. We suggest that this discrepancy occur because O VI 1038 $\AA$ is located very near the $\mathrm{C}$ II line at $1037 \AA$, and thus the analysis may be influenced by the procedure of line fitting. A similar discrepancy can be also found for the $\mathrm{C}$ II and $\mathrm{N}$ $\mathrm{V}$ doublets. The intensity of both lines in $\mathrm{CH}$ is measured to be about $40 \%$ lower than in QS.

Table 6.3: Derived line parameters for the O VI line

\begin{tabular}{|c|c|c|c|c|c|}
\hline Region & Pixel & $\begin{array}{l}\text { Dispersion } \\
\text { (mÅ/pixel) }\end{array}$ & $\begin{array}{l}\text { Doppler shift } \\
\left(\mathrm{km} \mathrm{s}^{-1}\right)\end{array}$ & $\begin{array}{c}\text { Intensity } \\
\left(\mathrm{mW} \mathrm{sr} \mathrm{sr}^{-1} \mathrm{~m}^{-2}\right)\end{array}$ & $\begin{array}{l}\text { Non-thermal v. } \\
\left(\mathrm{km} \mathrm{s}^{-1}\right)\end{array}$ \\
\hline $\mathrm{CH} 6(\mathrm{R})(1)$ & AK 571 & 44.16 & $5.8 \pm 2.2$ & $223.2 \pm 7.0$ & $37.0 \pm 0.8$ \\
\hline $\mathrm{CH} \overline{6}(\mathrm{R})(2)$ & AK 701 & 44.16 & $8.5 \pm 2.1$ & $100.1 \pm 5.0$ & $34.6 \pm 1.0$ \\
\hline QS $1(\mathrm{R})(1)$ & AK 571 & 44.16 & $7.5 \pm 1.9$ & $363.0 \pm 9.9$ & $36.6 \pm 0.7$ \\
\hline QS1 $(\mathrm{R})(2)$ & AK 701 & 44.16 & $10.5 \pm 1.8$ & $161.6 \pm 7.4$ & $34.7 \pm 0.7$ \\
\hline $\mathrm{CH} 11(1)$ & AB 208 & 43.94 & $3.2 \pm 1.6$ & $237.3 \pm 8.2$ & $38.9 \pm 0.8$ \\
\hline $\mathrm{CH} \overline{11}(2)$ & AK 337 & 43.94 & $5.4 \pm 1.7$ & $106.4 \pm 4.0$ & $35.6 \pm 1.0$ \\
\hline $\mathrm{CH}=(\mathrm{R})(1)$ & AK 571 & 44.15 & $-0.2 \pm 1.8$ & $188.6 \pm 3.4$ & $41.2 \pm 0.6$ \\
\hline $\mathrm{CH} \overline{13}(\mathrm{R})(2)$ & AK 700 & 44.15 & $2.7 \pm 1.9$ & $90.3 \pm 4.2$ & $39.4 \pm 0.8$ \\
\hline
\end{tabular}

(1) $1031.926 \AA$; (2) $1037.617 \AA$. 


\section{O V at $629.730 \AA$}

The O V line at $629.730 \AA$ was observed in 2 nd order on the bare part of the detector. The rest wavelength is adopted from Kelly (1987). The line is believed to be blended by a S II line (1259.53 $\AA$ ). In the $\mathrm{KBr}$ portion of the detector, the $\mathrm{O} \mathrm{V}$ line is estimated to be approximately 3-6 times strong than the blending line (Brekke et al., 1997; Teriaca et al., 1999). From our data, when it was recorded in the $\mathrm{KBr}$ portion of the detector, its Doppler shift is found to be around $14 \mathrm{~km} \mathrm{~s}^{-1}$ for all three regions listed in Table 6.4, a value near the $16 \mathrm{~km} \mathrm{~s}^{-1}$ observed by Brekke et al. (1997). Compared with the Doppler shift deduced from the bare portion that is listed in the table, the value deduced from the $\mathrm{KBr}$ portion seems to be obviously affected by the blending line.

If recorded in the bare part, Teriaca et al. (1999) estimated that the O V line is about 22 times stronger than the blend, and thus its line position should be less influenced. Hence, we used the data obtained from the bare portion for this study. Three chromospheric lines (C I at 1257.5649 $\AA$, Si I at 1258.7950 and S I at $1262.8596 \AA$ ) are used as reference lines. The results obtained in two CHs and QS region are shown in Table 6.4.

The Doppler shift of this line measured in QS region is falling in the range obtained by other authors (Peter and Judge, 1999; Teriaca et al., 1999), and about $2.5 \mathrm{~km} \mathrm{~s}^{-1}$ larger than in $\mathrm{CH}$ region. A 55\% lower intensity and slightly larger line width can be found in $\mathrm{CH} 6$ than in QS1.

Table 6.4: Derived line parameters for the O v line

\begin{tabular}{l|c|c|c|c|c}
\hline Region & Pixel & $\begin{array}{c}\text { Dispersion } \\
(\mathrm{mÅ/pixel})\end{array}$ & $\begin{array}{c}\text { Doppler shift } \\
\left(\mathrm{km} \mathrm{s}^{-1}\right)\end{array}$ & $\begin{array}{c}\text { Intensity } \\
\left(\mathrm{mW} \mathrm{sr}^{-1} \mathrm{~m}^{-2}\right)\end{array}$ & $\begin{array}{c}\text { Non-thermal v. } \\
\left(\mathrm{km} \mathrm{s}^{-1}\right)\end{array}$ \\
\hline CH6(R) & AB 150 & 43.11 & $5.5 \pm 1.7$ & $369.7 \pm 25.0$ & $30.2 \pm 1.0$ \\
QS11(R) & AB 150 & 43.09 & $8.2 \pm 1.7$ & $839.3 \pm 28.6$ & $29.3 \pm 0.7$ \\
CH13(R) & AB 154 & 42.97 & $6.0 \pm 1.9$ & $561.8 \pm 22.8$ & $31.8 \pm 0.8$ \\
\hline
\end{tabular}

\section{S VI at $933 \AA$}

The S VI line at $933.380 \AA$ can be measured in several spectral windows obtained in different regions listed in Table 6.5. The rest wavelength is taken from Kaufman and $\operatorname{Martin}(1993)$. Three O I lines at 929.517 $\AA, 932.225 \AA$ and $935.193 \AA$ near this line can serve as reference lines.

In Table 6.5, the Doppler shifts deduced from "raster scan" mode can be found to be apparently larger than those obtained from the "reference spectrum", and also obviously higher than those of other transition region lines formed at a similar temperature level. At present, we have not found a reason responsible for such a discrepancy. 
Table 6.5: Derived line parameters for the S VI line

\begin{tabular}{|c|c|c|c|c|c|}
\hline Region & Pixel & $\begin{array}{l}\text { Dispersion } \\
\text { (mÅ/pixel) }\end{array}$ & $\begin{array}{c}\text { Doppler shift } \\
\left(\mathrm{km} \mathrm{s}^{-1}\right)\end{array}$ & $\begin{array}{c}\text { Intensity } \\
\left(\mathrm{mW} \mathrm{sr}{ }^{-1} \mathrm{~m}^{-2}\right)\end{array}$ & $\begin{array}{c}\text { Non-thermal v. } \\
\left(\mathrm{km} \mathrm{s}^{-1}\right)\end{array}$ \\
\hline $\mathrm{CH} 6(\mathrm{R})$ & AK 361 & 44.24 & $7.4 \pm 1.8$ & $9.8 \pm 0.6$ & $35.3 \pm 1.4$ \\
\hline CH馬(R) & AK 650 & 44.42 & $8.7 \pm 1.7$ & $11.4 \pm 0.4$ & $36.8 \pm 0.8$ \\
\hline $\mathrm{CH} \overline{\overline{6}}$ & AK 722 & 44.57 & $15.5 \pm 1.6$ & $11.1 \pm 0.4$ & $34.4 \pm 0.6$ \\
\hline $\mathrm{CH} \overline{\overline{6}}(\mathrm{R})$ & AB 937 & 44.48 & $7.6 \pm 1.7$ & $11.3 \pm 0.9$ & $36.1 \pm 1.4$ \\
\hline QS1(R) & AK 361 & 44.26 & $10.4 \pm 1.9$ & $12.8 \pm 0.6$ & $33.3 \pm 1.0$ \\
\hline QS1 $1(\mathrm{R})$ & AK 650 & 44.43 & $13.0 \pm 2.3$ & $13.3 \pm 0.7$ & $32.4 \pm 1.3$ \\
\hline QS高 & AK 722 & 44.53 & $18.3 \pm 1.9$ & $12.3 \pm 0.5$ & $32.8 \pm 0.8$ \\
\hline QS1 $1(\mathrm{R})$ & AB 937 & 44.56 & $14.4 \pm 1.8$ & $15.9 \pm 1.8$ & $32.4 \pm 1.4$ \\
\hline $\mathrm{CH} \cdot 13(\mathrm{R})$ & AK 644 & 44.34 & $7.2 \pm 1.7$ & $8.6 \pm 0.3$ & $37.2 \pm 0.8$ \\
\hline
\end{tabular}

\section{S V at $786 \AA$}

The rest wavelength of the S V line was reported to be $786.470 \AA$ (Kaufman and Martin, 1993 ) and $786.480 \AA$ (Kelly, 1987), which is equivalent to a difference in velocity of $3.8 \mathrm{~km} \mathrm{~s}^{-1}$. The results listed in Table 6.6 are derived by using the rest wavelength of $786.480 \AA$, which was observed in 2 nd order of the diffraction. Three chromospheric lines (the Fe II lines at 1569.674 $\AA$ and 1570.244 $\AA$, and the Si I line at $1578.478 \AA$ ) are used as reference lines. The derived line parameters are listed in Table 6.6.

Table 6.6: Derived line parameters for the $S$ V line

\begin{tabular}{l|c|c|c|c|c}
\hline Region & Pixel & $\begin{array}{c}\text { Dispersion } \\
(\mathrm{m} \AA / \text { pixel })\end{array}$ & $\begin{array}{c}\text { Doppler shift } \\
\left(\mathrm{km} \mathrm{s}^{-1}\right)\end{array}$ & $\begin{array}{c}\text { Intensity } \\
\left(\mathrm{mW} \mathrm{sr}^{-1} \mathrm{~m}^{-2}\right)\end{array}$ & $\begin{array}{c}\text { Non-thermal v. } \\
\left(\mathrm{km} \mathrm{s}^{-1}\right)\end{array}$ \\
\hline CH6(R) & AK 377 & 41.70 & $8.8 \pm 1.8$ & $31.4 \pm 1.0$ & $26.4 \pm 0.6$ \\
QS1(R) & AK 377 & 41.65 & $10.4 \pm 1.6$ & $55.0 \pm 1.4$ & $24.2 \pm 0.4$ \\
CH13(R) & AK 390 & 41.66 & $5.2 \pm 1.6$ & $55.5 \pm 1.6$ & $24.7 \pm 0.4$ \\
\hline
\end{tabular}

\section{N V at $1238 \AA$ and $1242 \AA$}

To measure the Doppler shift of these two resonance lines of $\mathrm{N} \mathrm{V}$ is difficult due to a lack of reliable reference lines very near them. Only some $\mathrm{C}$ I lines on the red side of them can be used. Fortunately, because they were recorded on the center of the detector in our data, we may assume that there is a nearly constant dispersion relation on this central part of the detector. Therefore, we try to use three C I lines (1244.535 $\AA, 1245.538 \AA$ and 1249.004 $\AA$ ), whose positions can be well fitted, to determine the dispersion and the absolute shift 
of the N V lines. The rest wavelengths of $1238.821 \AA$ and $1242.804 \AA$ are adopted from Kelly (1987). They have a deviation of $5.1 \mathrm{~km} \mathrm{~s}^{-1}$ and $6.3 \mathrm{~km} \mathrm{~s}^{-1}$ from those reported by Edlén (1934) (1238.800 $\AA$ and $1242.778 \AA$ ). From Table 6.7, we find that the two lines have a relatively consistent Doppler shift with a departure of less than $2 \mathrm{~km} \mathrm{~s}^{-1}$, which may imply that the average Doppler shift measured in the two lines is reasonable.

Table 6.7: Derived line parameters for the N V line

\begin{tabular}{|c|c|c|c|c|c|}
\hline Region & Pixel & $\begin{array}{l}\text { Dispersion } \\
\text { (mÅ/pixel) }\end{array}$ & $\begin{array}{c}\text { Doppler shift } \\
\left(\mathrm{km} \mathrm{s}^{-1}\right)\end{array}$ & $\begin{array}{c}\text { Intensity } \\
\left(\mathrm{mW} \mathrm{sr}{ }^{-1} \mathrm{~m}^{-2}\right)\end{array}$ & $\begin{array}{l}\text { Non-thermal v. } \\
\left(\mathrm{km} \mathrm{s}^{-1}\right)\end{array}$ \\
\hline $\mathrm{CH} 6(1)$ & AK 524 & 43.40 & $5.1 \pm 1.6$ & $50.9 \pm 1.1$ & $33.0 \pm 0.3$ \\
\hline $\mathrm{CH} \overline{\overline{6}}(2)$ & AK 616 & 43.40 & $6.6 \pm 1.7$ & $27.0 \pm 1.1$ & $32.2 \pm 0.8$ \\
\hline $\mathrm{CH} \overline{6}(\mathrm{R})(1)$ & AK 552 & 43.21 & $7.1 \pm 1.6$ & $27.7 \pm 1.2$ & $31.3 \pm 0.8$ \\
\hline $\mathrm{CH} \overline{\overline{6}}(\mathrm{R})(2)$ & AK 644 & 43.21 & $7.7 \pm 2.1$ & $14.5 \pm 1.6$ & $27.9 \pm 1.8$ \\
\hline QS11 1 (1) & AK 524 & 43.35 & $13.0 \pm 1.6$ & $77.9 \pm 3.6$ & $32.1 \pm 0.8$ \\
\hline QS馬(2) & AK 616 & 43.35 & $12.7 \pm 1.7$ & $40.0 \pm 2.4$ & $32.0 \pm 1.1$ \\
\hline QS言 $(\mathrm{R})(1)$ & AK 552 & 43.17 & $11.6 \pm 1.7$ & $86.7 \pm 2.9$ & $29.2 \pm 0.7$ \\
\hline QS六(R)(2) & AK 644 & 43.17 & $11.9 \pm 1.7$ & $41.4 \pm 2.1$ & $26.4 \pm 1.7$ \\
\hline $\mathrm{CH} 13(\mathrm{R})(1)$ & AK 556 & 43.32 & $4.7 \pm 1.7$ & $51.5 \pm 2.9$ & $37.7 \pm 1.3$ \\
\hline $\mathrm{CH} \overline{13}(\mathrm{R})(2)$ & AK 648 & 43.32 & $6.5 \pm 2.1$ & $24.8 \pm 1.7$ & $33.1 \pm 1.7$ \\
\hline
\end{tabular}

(1) $1238.821 \AA$; (2) $1242.804 \AA$.

\section{IV at $1548 \AA$ and $1550 \AA$}

Two resonance lines of C IV at $1548.204 \AA$ and $1550.781 \AA$ were observed in the same spectral window as the Ne VIII line. New rest wavelengths are adopted from a recent work done by Griesmann and Kling (2000) and are very close those (1548.202 $\AA$ and $1550.774 \AA$ ) listed in Kelly (1987). The wavelength calibration is made by using the $\mathrm{C}$ I line (1542.1766 $\AA$ ) and the Fe II line (1550.274 $\AA$ ). From Table 6.10, very consistent results are obtained for each line in both $\mathrm{CH} 6$ and QS1. A $15 \%$ lower intensity and $1 \mathrm{~km}$ $\mathrm{s}^{-1}$ larger non-thermal velocity can be found in the $\mathrm{CH}$ region.

\section{N IV at $765 \AA$}

The N IV line at $765.148 \AA$ (Kelly, 1987) was observed in 2nd order. The reference lines used to make a wavelength calibration are two Si II lines at $1526.7076 \AA$ and 1533.4320 A. From Table 6.9, this line has a small red shift obtained in CHs. However, it should be mentioned that the reference lines of Si II themselves have a red shift of about $2 \mathrm{~km} \mathrm{~s}^{-1}$ relative to the S I and C I lines. Hence, if calibrated to these lines, its Doppler shift will 
Table 6.8: Derived line parameters for the C IV line

\begin{tabular}{|c|c|c|c|c|c|}
\hline Region & Pixel & $\begin{array}{l}\text { Dispersion } \\
\text { (mÅ/pixel) }\end{array}$ & $\begin{array}{c}\text { Doppler shift } \\
\left(\mathrm{km} \mathrm{s}^{-1}\right)\end{array}$ & $\begin{array}{c}\text { Intensity } \\
\left(\mathrm{mW} \mathrm{sr}{ }^{-1} \mathrm{~m}^{-2}\right)\end{array}$ & $\begin{array}{c}\text { Non-thermal v. } \\
\qquad\left(\mathrm{km} \mathrm{s}^{-1}\right)\end{array}$ \\
\hline $\mathrm{CH} 6(1)$ & AK 660 & 41.83 & $5.9 \pm 1.7$ & $438.5 \pm 7.7$ & $26.0 \pm 0.4$ \\
\hline $\mathrm{CH} \overline{\overline{6}}(2)$ & AK 721 & 41.83 & $5.8 \pm 1.9$ & $208.5 \pm 7.5$ & $26.3 \pm 0.6$ \\
\hline QS1(1) & AK 660 & 41.82 & $7.9 \pm 1.7$ & $524.4 \pm 4.8$ & $24.9 \pm 0.2$ \\
\hline QS1] & AK 721 & 41.82 & $7.4 \pm 1.9$ & $242.8 \pm 9.4$ & $25.4 \pm 0.7$ \\
\hline
\end{tabular}

(1) $1548.204 \AA$; (2) $1550.781 \AA$.

be $4.8 \mathrm{~km} \mathrm{~s}^{-1}, 7.8 \mathrm{~km} \mathrm{~s}^{-1}$ and $3.2 \mathrm{~km} \mathrm{~s}^{-1}$ for $\mathrm{CH} 6$, QS1 and $\mathrm{CH} 13$, respectively. The intensity of this line is found to be $50 \%$ weaker in $\mathrm{CH} 6$ than in QS1.

Table 6.9: Derived line parameters for the N IV line

\begin{tabular}{l|c|c|c|c|c}
\hline Region & Pixel & $\begin{array}{c}\text { Dispersion } \\
(\mathrm{m} \AA / \text { pixel })\end{array}$ & $\begin{array}{c}\text { Doppler shift } \\
\left(\mathrm{km} \mathrm{s}^{-1}\right)\end{array}$ & $\begin{array}{c}\text { Intensity } \\
\left(\mathrm{mW} \mathrm{sr}^{-1} \mathrm{~m}^{-2}\right)\end{array}$ & $\begin{array}{c}\text { Non-thermal v. } \\
\left(\mathrm{km} \mathrm{s}^{-1}\right)\end{array}$ \\
\hline CH6(R) & AK 571 & 42.00 & $2.8 \pm 1.7$ & $53.1 \pm 1.8$ & $24.7 \pm 0.6$ \\
QS11(R) & AK 571 & 42.02 & $5.8 \pm 1.7$ & $101.9 \pm 2.0$ & $23.7 \pm 0.3$ \\
CH13(R) & AK 578 & 41.95 & $1.2 \pm 1.6$ & $91.0 \pm 3.3$ & $24.7 \pm 0.7$ \\
\hline
\end{tabular}

\section{O IV at $1401 \AA$ and $788 \AA$}

The O IV lines at $1401.156 \AA$ and $787.711 \AA$ (in 2 nd order) were recorded in two different spectral windows. For the O IV $1401.156 \AA$, the reference lines are the equivalent of those of the two Si IV lines (1393.755 $\AA$ and $1402.770 \AA$ ). Three chromospheric lines serve as reference lines for the O IV line at $787.711 \AA$, which include the Fe II lines (1569.674 $\AA$ and $1570.244 \AA$ ) and the Si I line (1578.478 $\AA$ ). The results are listed in Table 6.10 . It should be mentioned that another O IV line at $790.199 \AA$ is not used, which shows a considerably smaller red shift and broader line width, as also found by Brekke et al. (1997). By inspection of the literature (Kelly, 1987), this line is obviously blended by another O IV line at $790.109 \AA$, so that the measured line profile is actually contributed by two lines.

\section{Si IV at $1394 \AA ̊$ and $1403 \AA$}

Two Si IV lines were recorded in a same spectral window around $1400 \AA$. Their rest wavelengths of $1393.755 \AA$ and $1402.770 \AA$ are taken from Kelly (1987). Four chromospheric lines, Fe II at 1392.817 $\AA$, S I at 1396.1121 $\AA, 1401.5135 \AA$ and $1409.337 \AA$ are used to 
Table 6.10: Derived line parameters for the O IV line

\begin{tabular}{l|c|c|c|c|c}
\hline Region & Pixel & $\begin{array}{c}\text { Dispersion } \\
(\mathrm{m} \AA / \text { pixel })\end{array}$ & $\begin{array}{c}\text { Doppler shift } \\
\left(\mathrm{km} \mathrm{s}^{-1}\right)\end{array}$ & $\begin{array}{c}\text { Intensity } \\
\left(\mathrm{mW} \mathrm{sr}^{-1} \mathrm{~m}^{-2}\right)\end{array}$ & $\begin{array}{c}\text { Non-thermal v. } \\
\left(\mathrm{km} \mathrm{s}^{-1}\right)\end{array}$ \\
\hline CH6(R)(1) & AK 502 & 42.64 & $7.5 \pm 1.8$ & $15.7 \pm 1.1$ & $29.6 \pm 1.3$ \\
CH/(R)(2) & AK 436 & 41.70 & $5.4 \pm 1.8$ & $57.5 \pm 2.2$ & $24.6 \pm 0.7$ \\
QS11(R)(1) & AK 502 & 42.65 & $10.7 \pm 1.8$ & $38.7 \pm 3.0$ & $24.9 \pm 1.0$ \\
QS1/(R)(2) & AK 436 & 41.65 & $6.9 \pm 1.6$ & $103.4 \pm 2.5$ & $24.2 \pm 0.4$ \\
CH13(R)(1) & AK 505 & 42.63 & $6.8 \pm 1.9$ & $27.4 \pm 1.0$ & $27.6 \pm 0.7$ \\
CH13(R)(2) & AK 449 & 41.66 & $4.1 \pm 1.6$ & $96.6 \pm 2.3$ & $23.1 \pm 0.6$ \\
\hline
\end{tabular}

(1) $1401.156 \AA$; (2) $787.711 \AA$ in 2 nd order.

determine the dispersion relation and the Doppler shift. Table 6.11 shows the derived line parameters for the two lines.

Table 6.11: Derived line parameters for the Si IV line

\begin{tabular}{|c|c|c|c|c|c|}
\hline Region & Pixel & $\begin{array}{l}\text { Dispersion } \\
\text { (mÅ/pixel) }\end{array}$ & $\begin{array}{c}\text { Doppler shift } \\
\left(\mathrm{km} \mathrm{s}^{-1}\right)\end{array}$ & $\begin{array}{c}\text { Intensity } \\
\left(\mathrm{mW} \mathrm{sr}{ }^{-1} \mathrm{~m}^{-2}\right)\end{array}$ & $\begin{array}{l}\text { Non-thermal v. } \\
\left(\mathrm{km} \mathrm{s}^{-1}\right)\end{array}$ \\
\hline $\mathrm{CH} 6(\mathrm{R})(1)$ & AK 328 & 42.64 & $6.1 \pm 1.7$ & $194.5 \pm 5.4$ & $30.1 \pm 0.6$ \\
\hline $\mathrm{CH} \overline{\overline{6}}(\mathrm{R})(2)$ & AK 540 & 42.64 & $4.7 \pm 1.9$ & $98.3 \pm 6.4$ & $28.6 \pm 1.1$ \\
\hline 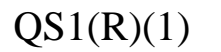 & AK 328 & 42.65 & $7.6 \pm 1.9$ & $310.2 \pm 16.1$ & $25.8 \pm 0.7$ \\
\hline QS言(R)(2) & AK 540 & 42.65 & $7.7 \pm 1.9$ & $162.2 \pm 11.6$ & $27.1 \pm 0.8$ \\
\hline $\mathrm{CH} 13(\mathrm{R})(1)$ & AK 331 & 42.63 & $4.7 \pm 1.9$ & $249.3 \pm 10.3$ & $26.2 \pm 0.7$ \\
\hline $\mathrm{CH} \overline{\overline{13}}(\mathrm{R})(2)$ & AK 542 & 42.63 & $3.7 \pm 2.0$ & $133.4 \pm 12.5$ & $29.1 \pm 1.4$ \\
\hline
\end{tabular}

(1) $1393.755 \AA$; (2) $1402.770 \AA$.

\section{II at $1036 \AA ̊$ and $1037 \AA$}

In the spectral window around $1030 \AA$ together with the two O VI lines, the $\mathrm{C}$ II lines at $1036.3367 \AA$ and $1037.0182 \AA$ (Kaufman and Edlén, 1974) are also very strong with a high signal-to-noise ratio. They are formed at a temperature of about $0.05 \mathrm{MK}$. The reference lines used for these two lines are the same as the ones for $\mathrm{O}$ VI lines. The results obtained in three regions are shown in Table 6.12.

As we have discussed, the $\mathrm{C}$ II line at $1037 \AA$ is very near the blue side of the $\mathrm{O}$ VI line at $1038 \AA$, thus the measurement of the line width is possibly affected by such a position. This may be responsible for a systematically larger non-thermal velocity deduced for this 
line than for the C II line at $1036 \AA$. For the Doppler shift, a discrepancy of the two lines is measured to be $1-2 \mathrm{~km} \mathrm{~s}^{-1}$, relatively smaller than for the two $\mathrm{O}$ VI lines. In addition, no pronounced difference of the Doppler shift is found between $\mathrm{CH} 6$ and QS1 if considering the uncertainty of measurements. Furthermore, $\mathrm{CH} 6$ exhibits a marginally stronger intensity than QS1.

Table 6.12: Derived line parameters for the C II line

\begin{tabular}{|c|c|c|c|c|c|}
\hline Region & Pixel & $\begin{array}{l}\text { Dispersion } \\
\text { (mÅ/pixel) }\end{array}$ & $\begin{array}{c}\text { Doppler shift } \\
\left(\mathrm{km} \mathrm{s}^{-1}\right)\end{array}$ & $\begin{array}{c}\text { Intensity } \\
\left(\mathrm{mW} \mathrm{sr} \mathrm{s}^{-1} \mathrm{~m}^{-2}\right)\end{array}$ & $\begin{array}{c}\text { Non-thermal v. } \\
\left(\mathrm{km} \mathrm{s}^{-1}\right)\end{array}$ \\
\hline $\mathrm{CH} 6(\mathrm{R})(1)$ & AK 671 & 44.16 & $5.2 \pm 2.1$ & $53.2 \pm 3.2$ & $26.9 \pm 0.8$ \\
\hline $\mathrm{CH} \overline{6}(\mathrm{R})(2)$ & AK 687 & 44.16 & $6.4 \pm 2.0$ & $62.6 \pm 1.4$ & $30.6 \pm 0.6$ \\
\hline QS商(R)(1) & AK 671 & 44.16 & $4.9 \pm 2.1$ & $49.9 \pm 3.9$ & $24.4 \pm 1.0$ \\
\hline QS司(R)(2) & AK 687 & 44.16 & $5.9 \pm 1.7$ & $59.5 \pm 1.4$ & $26.8 \pm 0.4$ \\
\hline $\mathrm{CH} 11(1)$ & AK 308 & 43.94 & $4.1 \pm 1.6$ & $45.8 \pm 0.5$ & $29.3 \pm 0.3$ \\
\hline $\mathrm{CH} \overline{11}(2)$ & AK 324 & 43.94 & $6.1 \pm 1.6$ & $55.1 \pm 1.9$ & $31.2 \pm 0.8$ \\
\hline $\mathrm{CH} \overline{13}(\mathrm{R})(1)$ & AK 671 & 44.15 & $4.9 \pm 1.8$ & $61.5 \pm 1.8$ & $28.8 \pm 0.4$ \\
\hline $\mathrm{CH} \overline{\overline{13}}(\mathrm{R})(2)$ & AK 686 & 44.15 & $4.7 \pm 1.8$ & $73.9 \pm 1.3$ & $30.9 \pm 0.4$ \\
\hline
\end{tabular}

(1) $1036.337 \AA$; (2) $1037.018 \AA$.

\subsubsection{Chromospheric lines}

The chromospheric lines, such as S I, Si I, Fe II, C I, O I and Si II, are frequently used as reference lines to determine the Doppler shift of other lines formed at a higher temperature. The S I and Si I lines are believed to be formed at a temperature below $10000 \mathrm{~K}$ and are assumed to be rest (see, e.g. Chae et al., 1998c). The other lines listed above have formation temperatures between $10000 \mathrm{~K}$ to $20000 \mathrm{~K}$. Using SUMER data, Chae et al. (1998c) suggested that they have a redshift of $1.5-2.6 \mathrm{~km} \mathrm{~s}^{-1}$ relative to S I and Si I. In the QS region, Teriaca et al. (1999) measured such relative Doppler shifts to be around 0 $\mathrm{km} \mathrm{s}^{-1}$ for the Fe II lines, $-0.1-1.3 \mathrm{~km} \mathrm{~s}^{-1}$ for the O I lines and $1.8 \mathrm{~km} \mathrm{~s}^{-1}$ for the $\mathrm{Si}$ II line, respectively.

\section{Si II}

For the Si II lines, the measurement is carried out in our study in the spectral window around $1310 \AA$, in which a series of S I and C I lines can be used as reference lines. They include $\mathrm{S}$ I lines at $1302.8633 \AA$ and $1303.1105 \AA$, as well as $\mathrm{C}$ I lines at $1311.363 \AA$, 1311.924 $\AA$ and 1312.247 $\AA$. The rest wavelength of this Si II line is at 1304.3719 $\AA$. Another Si II line at $1533.432 \AA$ was recorded in the spectral window around $1540 \AA$ and 
used to measure the line intensity and width. All rest wavelengths are taken from Kelly (1987). The results are shown in Table 6.13.

As can be seen from Table 6.13, the Si II line at $1304 \AA$ has a Doppler shift of $2.3 \mathrm{~km}$ $\mathrm{s}^{-1}$ in both $\mathrm{CH}$ and QS regions. The non-thermal velocity for both lines is around $20 \mathrm{~km}$ $\mathrm{s}^{-1}$, in agreement with that measured by Teriaca et al. (1999). The intensity, if seen in Si II $1533.432 \AA$ that has much more data samples, is slightly higher in the $\mathrm{CH}$ region. Note that these lines may be affected by finite opacity, so the measured line widths may be larger than their real values.

Table 6.13: Derived line parameters for the Si II line

\begin{tabular}{l|c|c|c|c|c}
\hline Region & Pixel & $\begin{array}{c}\text { Dispersion } \\
(\mathrm{m} \AA / \text { pixel })\end{array}$ & $\begin{array}{c}\text { Doppler shift } \\
\left(\mathrm{km} \mathrm{s}^{-1}\right)\end{array}$ & $\begin{array}{c}\text { Intensity } \\
\left(\mathrm{mW} \mathrm{sr}^{-1} \mathrm{~m}^{-2}\right)\end{array}$ & $\begin{array}{c}\text { Non-thermal v. } \\
\left(\mathrm{km} \mathrm{s}^{-1}\right)\end{array}$ \\
\hline CH6(R)(1) & AK 311 & 43.00 & $2.3 \pm 2.0$ & $47.8 \pm 3.6$ & $22.0 \pm 1.3$ \\
CH6(2) & AK 306 & 41.90 & & $228.5 \pm 17.8$ & $23.7 \pm 1.1$ \\
QS11(R)(1) & AK 311 & 43.00 & $2.3 \pm 2.1$ & $52.3 \pm 5.7$ & $18.7 \pm 1.1$ \\
QS112) & AK 306 & 41.90 & & $203.1 \pm 11.7$ & $21.7 \pm 0.7$ \\
CH13(R)(1) & AK 315 & 43.00 & $1.8 \pm 1.9$ & $50.6 \pm 6.0$ & $20.7 \pm 1.3$ \\
\hline
\end{tabular}

(1) $1304.372 \AA$; (2) $1533.432 \AA$.

\section{O I}

The Doppler shift of the $\mathrm{O}$ I lines are measured in the spectral window around 1310 $\AA$, the same as for the Si II line at $1304 \AA$. The two O I lines have rest wavelengths of $1304.8575 \AA$ and $1306.0286 \AA$ (Kelly, 1987). Three lines in other spectral windows with rest wavelengths of $929.517 \AA, 1355.598 \AA$ and $1358.518 \AA$ are used to measure the intensity and line width. The results are shown in Table 6.14 .

From the results listed in Table 6.14, one can find that these lines exhibit a small Doppler shift relative to the $\mathrm{C}$ I line. The line width measured for these lines shows a larger discrepancy. The lines at $1356 \AA$ and $1359 \AA$ have a small value of non-thermal velocity around $10 \mathrm{~km} \mathrm{~s}^{-1}$, consistent with those measured by other authors (Chae et al., 1998a; Teriaca et al. , 1999), while the others have a larger value around $20 \mathrm{~km} \mathrm{~s}^{-1}$, which may suffer more from finite opacity than the former two. Again, they have a slightly larger intensity in $\mathrm{CH}$ than in QS regions.

\section{I and Fe II}

From our data, we find that the Fe II line at 1550.274 $\AA$ has an average Doppler shift of $-0.5 \pm 1.5 \mathrm{~km} \mathrm{~s}^{-1}$ relative to the Si I lines, in agreement with the result obtained 
Table 6.14: Derived line parameters for the O I line

\begin{tabular}{|c|c|c|c|c|c|}
\hline Region & Pixel & $\begin{array}{l}\text { Dispersion } \\
\text { (mÅ/pixel) }\end{array}$ & $\begin{array}{c}\text { Doppler shift } \\
\left(\mathrm{km} \mathrm{s}^{-1}\right)\end{array}$ & $\begin{array}{c}\text { Intensity } \\
\left(\mathrm{mW} \mathrm{sr} \mathrm{s}^{-1} \mathrm{~m}^{-2}\right)\end{array}$ & $\begin{array}{l}\text { Non-thermal v. } \\
\qquad\left(\mathrm{km} \mathrm{s}^{-1}\right)\end{array}$ \\
\hline $\mathrm{CH} 6(\mathrm{R})(1)$ & AK 322 & 43.00 & $1.8 \pm 1.7$ & $514.3 \pm 29.4$ & $25.1 \pm 0.7$ \\
\hline $\mathrm{CH} \overline{6}(\mathrm{R})(2)$ & AK 349 & 43.00 & $1.1 \pm 1.6$ & $548.6 \pm 18.8$ & $22.5 \pm 0.4$ \\
\hline $\mathrm{CH} \overline{6}(3)$ & AK 635 & 44.57 & & $2.1 \pm 1.5$ & $21.3 \pm 0.7$ \\
\hline $\mathrm{CH} \overline{\mathrm{6}}(\mathrm{R})(4)$ & AK 326 & 42.76 & & $84.4 \pm 10.1$ & $10.5 \pm 0.8$ \\
\hline $\mathrm{CH} \overline{\mathrm{6}}(\mathrm{R})(5)$ & AK 394 & 42.76 & & $24.8 \pm 2.7$ & $9.6 \pm 1.0$ \\
\hline QS1(R)(1) & AK 322 & 43.00 & $1.3 \pm 1.7$ & $737.8 \pm 34.5$ & $21.8 \pm 0.6$ \\
\hline QS言 $(R)(2)$ & AK 349 & 43.00 & $0.0 \pm 1.9$ & $773.9 \pm 40.8$ & $19.7 \pm 0.7$ \\
\hline QS言(3) & AK 635 & 44.53 & & $1.8 \pm 0.2$ & $21.6 \pm 1.5$ \\
\hline QS言(R)(4) & AK 326 & 42.76 & & $77.3 \pm 10.5$ & $10.9 \pm 1.1$ \\
\hline QS旬(R)(5) & AK 394 & 42.76 & & $22.1 \pm 2.7$ & $10.2 \pm 0.7$ \\
\hline $\mathrm{CH} 13(\mathrm{R})(1)$ & AK 326 & 43.00 & $1.1 \pm 1.7$ & $584.3 \pm 22.0$ & $21.4 \pm 0.6$ \\
\hline $\mathrm{CH} \overline{\overline{13}}(\mathrm{R})(2)$ & AK 353 & 43.00 & $0.0 \pm 1.7$ & $621.9 \pm 46.3$ & $19.2 \pm 0.6$ \\
\hline
\end{tabular}

(1) $1304.858 \AA$; (2) $1306.029 \AA$; (3) $929.517 \AA$; (4) $1355.598 \AA$; (5) $1358.518 \AA$.

by Teriaca et al. (1999). The non-thermal velocity measured in this line has a value of $10.2 \pm 1.0 \mathrm{~km} \mathrm{~s}^{-1}$ in $\mathrm{CH} 6$ and $10.8 \pm 0.8 \mathrm{~km} \mathrm{~s}^{-1}$ in QS1.

For the $\mathrm{C}$ I line at $1542.177 \AA$, the non-thermal velocity has been measured to be $8.9 \pm 0.6$ $\mathrm{km} \mathrm{s}^{-1}$ in $\mathrm{CH} 6$ and $9.7 \pm 0.6 \mathrm{~km} \mathrm{~s}^{-1}$ in QS1. Moreover, both regions have the same value of the intensity.

It is interesting to find that, if observed in the same spectral window, the line width of chromospheric lines such as Fe II and C I lines has a very clear positive correlation with the intensity. As suggested by Doschek et al. (2001), this phenomenon is possible due to an opacity effect.

\section{Continuum}

In Table 6.15, the intensities of the continuum in wavelengths between $910 \AA$ and $1540 \AA$ are given for both $\mathrm{CH} 6$ and QS1. At the wavelength around $1539 \AA$, there is no essential difference between the two regions. A slight enhancement of the continuum in the $\mathrm{CH}$ can be found in wavelengths around $1235 \AA$, $932 \AA$ and $912 \AA$. Thus, there is no problem to conclude that the chromosphere of the two regions is more or less similar, although a detailed difference still exists, for example, the intensity distribution as was discussed in the last section. It should be mentioned that this comparison is made between the coronal hole and a normal QS region, but not the surrounding area of the $\mathrm{CH}$, where a pronounced enhancement of the intensity for continua with a wavelength shorter than $1100 \AA$ can be found (Schühle et al. , 1999). As was pointed by these authors, the polar crown filament 
may influence the brightness of the emission. If compared with the surrounding area of the equatorial coronal hole, which is usually associated with an active region, we also find that the surrounding area exhibits apparently enhanced brightness of the continuum in different wavelengths.

Table 6.15: Derived intensity of the continuum

\begin{tabular}{c|c|c}
\hline $\begin{array}{c}\text { Wavelength } \\
(\AA)\end{array}$ & $\begin{array}{c}\text { Intensity }(\mathrm{CH}) \\
\left(\mathrm{mW} \mathrm{sr}^{-1} \mathrm{~m}^{-2} \AA^{-1}\right)\end{array}$ & $\begin{array}{c}\text { Intensity (QS1) } \\
\left(\mathrm{mW} \mathrm{sr}^{-1} \mathrm{~m}^{-2} \AA^{-1}\right)\end{array}$ \\
\hline 1539 & 178.5 & 178.8 \\
1235 & 23.9 & 22.1 \\
932 & 1.7 & 1.5 \\
912 & 49.5 & 47.7 \\
\hline
\end{tabular}

\subsection{Temperature dependence of intensity, Doppler shift and line width}

\subsubsection{Intensity ratio between the $\mathrm{CH}$ and QS regions}

From the results discussed above, an average intensity ratio of different lines, for comparison between the coronal hole and the QS region, can thus be derived. Since the observa-

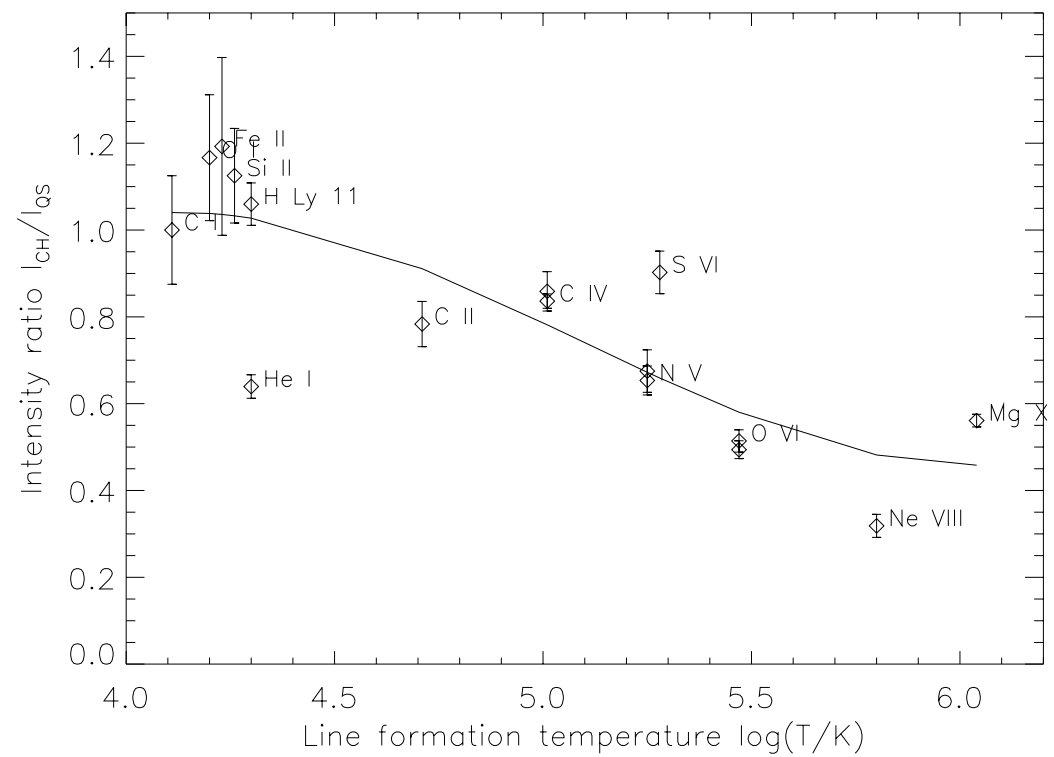

Figure 6.14: Line intensity ratio between $\mathrm{CH}$ and $\mathrm{QS}$ regions in various lines versus the line formation temperature. 
tions made in "reference spectrum" mode of SUMER have less data samples, and since the intensities are sensitive to the detailed structures pointed at by the slit, only the data obtained with the "raster scan" mode in CH6 and QS1 are used here to study the variation of the average radiance in different lines. The result is shown in Figure 6.14 with a scatter plot of the intensity ratio between the $\mathrm{CH}$ and QS regions for each line.

The trend of the intensity ratio varying with the formation temperature shown in Figure 6.14 is generally in agreement with previous observations (Wilhelm et al., 2002; Lemaire et al., 1999; Dere et al., 1989b; Huber et al., 1974; Stucki et al., 1999). However, it is worthwhile to emphasize here some additional interesting aspects. From Figure 6.14, the intensity ratio between the $\mathrm{CH}$ and QS region can be found to be close unity in the $\mathrm{C}$ I lines, rises to 1.1 to 1.2 for the other chromospheric lines (O I, Fe II and Si II) with a higher formation temperature of above $15000 \mathrm{~K}$, and then drops again in the transition region and coronal lines. An exception is the He I line that has only $65 \%$ of the QS brightness. In the transition region, one can find that the intensity ratio of the Li-like ions decrease with increasing formation temperature, but the S VI line shows a relatively small change in intensity between the two regions. As was discussed in Section 6.3.1. an apparent difference of the intensity distribution is also present for the Li-like ions of $\mathrm{N}^{4+}$ and $\mathrm{O}^{5+}$, for which we have suggested that it may be due to their wider formation temperature range.

Combined with the results of the continuum list in Table 6.15, it is interesting to note that the intensity ratio seen in the $\mathrm{C}$ I line and the continuum around $1539 \AA$ indicates that the low chromosphere is indeed the same for both $\mathrm{CH}$ and QS regions. But the evidence shows the upper chromosphere has an enhanced brightness in the $\mathrm{CH}$, as was also found by other authors in various chromospheric emission lines (Ly $\alpha$, Ly $\beta$, Ca II and Mg II, which are formed at temperatures of about or less than 20,000 K) (Gopalswamy et al., 1999), as well as in microwaves at frequencies between 15 and $39 \mathrm{GHz}$ (Bocchialini and Vial, 1996). However, because of a rather large uncertainty of our measurements, this result needs to be confirmed in future work.

\subsubsection{Average Doppler shift}

A temperature dependence of the average Doppler shift is plotted for $\mathrm{CH} 6$ and QS 1 in Figure 6.15. In Figure 6.15, the upper and middle panels show the average Doppler shift varying with the formation temperature in the $\mathrm{CH}$ and QS region, respectively, while the difference of the Doppler shift between the two regions measured in each line is illustrated in the bottom panel. The curves plotted as solid lines in each panel show the trend of the variation.

Except for the Ne VIII and $\mathrm{Mg} X$ lines, all other transition region lines appear on average red shifted in both $\mathrm{CH}$ and QS regions. Average red shifts of the transition region lines range mainly from $5 \mathrm{~km} \mathrm{~s}^{-1}$ to $10 \mathrm{~km} \mathrm{~s}^{-1}$ in the $\mathrm{CH}$ region, and $5 \mathrm{~km} \mathrm{~s}^{-1}$ to $15 \mathrm{~km}$ $\mathrm{s}^{-1}$ in the QS region. Small Doppler shifts are measured for the $\mathrm{Ne}$ VIII and $\mathrm{Mg} \mathrm{X}$ lines 

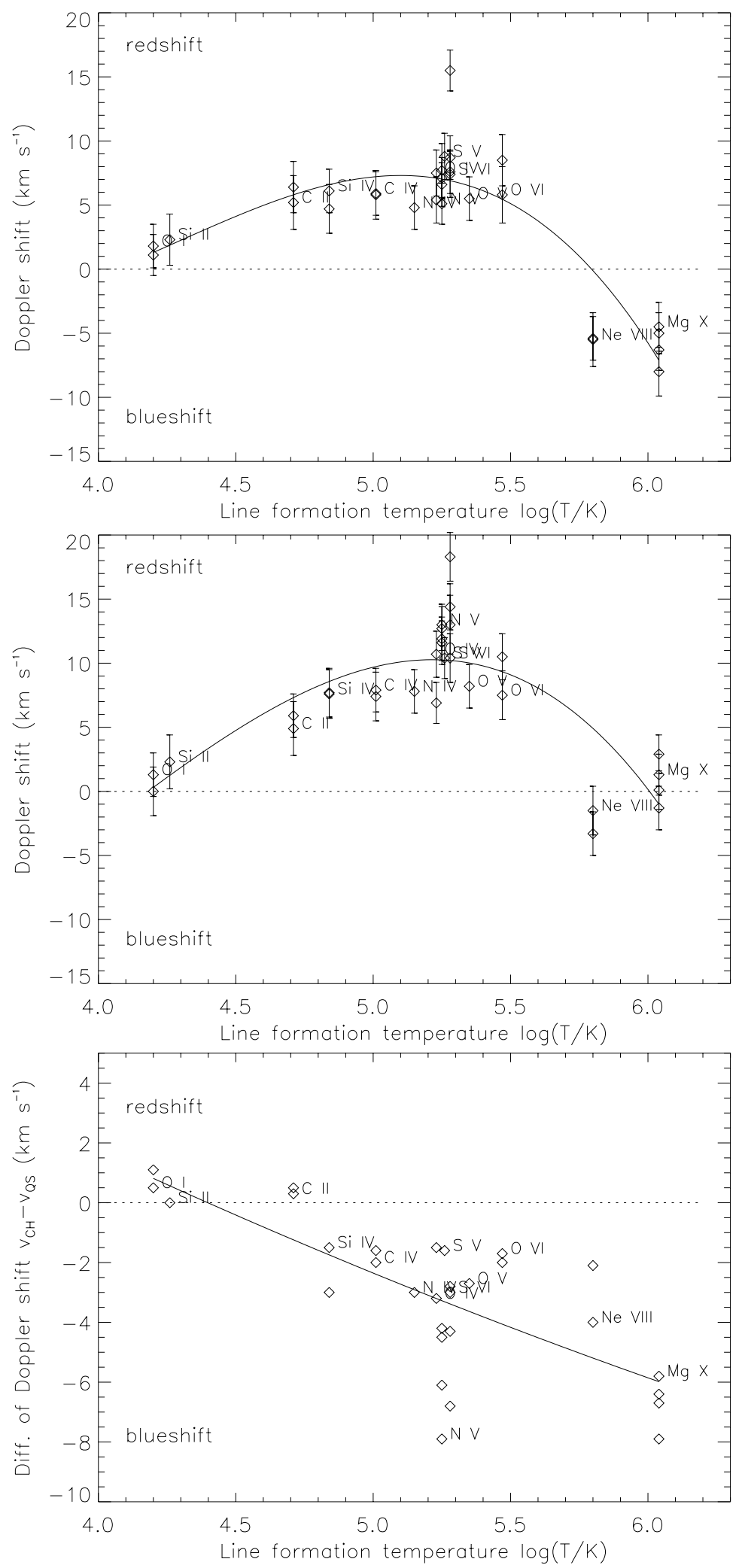

Figure 6.15: Average Doppler shift in various lines versus the formation temperature in $\mathrm{CHs}$ and QS. (a) $\mathrm{CH}$; (b) QS; (c) Difference of the Doppler shift between the $\mathrm{CH}$ and QS regions 
in the QS region. If considering that most of the emission of the two lines is constrained to magnetic loops in the QS region, these results are reasonable. On the other hand, both the $\mathrm{Ne}$ VIII and $\mathrm{Mg} \mathrm{X}$ lines have average blue shift in the $\mathrm{CH}$ region. The temperature dependence of the average Doppler shifts, in both the $\mathrm{CH}$ and QS regions, are consistent with previous results obtained by (Chae et al., 1998c; Peter and Judge, 1999; Teriaca et al. (1999), i.e., the red shift increases first with increasing line formation temperature, but then drops again at a temperature of about $0.3 \mathrm{MK}$ (at the $\mathrm{O}$ VI line).

The difference of the Doppler shift between the $\mathrm{CH}$ and QS regions also shows a temperature-dependent property. For chromospheric lines Si II and O I, such a difference is very small (within $1 \mathrm{~km} \mathrm{~s}^{-1}$ and rather small if considering the measurement errors). Except for the C II lines, transition region lines have systematically smaller red shifts in $\mathrm{CHs}$ than in the QS region. It should be emphasized that this relative Doppler shift is derived for each line with the same wavelength, which was recorded in a same pixel address, and calibrated by same reference lines for the $\mathrm{CH}$ and QS region. So the different shift should be more reliable because in this case only the fitting error will influence the accuracy of the measurements. The result obtained in this study is in agreement with that obtained in the PCHs (Stucki et al., 2000b). Such a difference of the Doppler shift may be important to consider the mass balance in the transition region of the $\mathrm{CH}$ and QS regions (Pneuman and Kopp, 1978), as was discussed in Section 6.3.3.

\subsubsection{Average line width}

In Figure 6.16, the average non-thermal velocity deduced for CH6 and QS1 is plotted versus the line formation temperature. For the QS region, the trend of non-thermal velocity varying with the temperature is similar to that found by other authors (Chae et al., 1998a; Teriaca et al., 1999), who also used the data obtained by SUMER. However, both results obtained by Teriaca et al. (1999) and our data exhibit a generally larger value than those of Chae et al. (1998a). As pointed by Teriaca et al. (1999), the instrumental broadening estimated by Chae et al. (1998a) is higher than that used in the standard software in the SUMER archive. In addition, our data show a larger non-thermal velocity of the $\mathrm{N} \mathrm{v,} \mathrm{S}$ VI and O VI lines. As we have mentioned in Section 6.4.2, these lines exhibit an obvious second component in their line profiles, which may lead to a broadening of the fitted line profile because we could not deduce them separately. Another possible reason may be that our data were obtained during the solar maximum, while the observations studied by other authors mentioned above were obtained near the solar minimum. This is indeed true for the $\mathrm{Mg} \mathrm{X}$ line, as will be discussed in the following.

For the line width in the $\mathrm{CH}$ region, the variation trend of lines formed below the upper transition region is more or less similar to that in the QS region. The difference between the two regions is small, with a larger value of around $1-2 \mathrm{~km} \mathrm{~s}^{-1}$ in the hole. However, an apparent difference arises for the Ne VIII and Mg X lines. It should be noted that we just compare them in two regions observed within several days during the solar maximum. In other coronal holes, as shown in Table 6.1, the line width of the $\mathrm{Mg} \mathrm{X}$ line shows very 

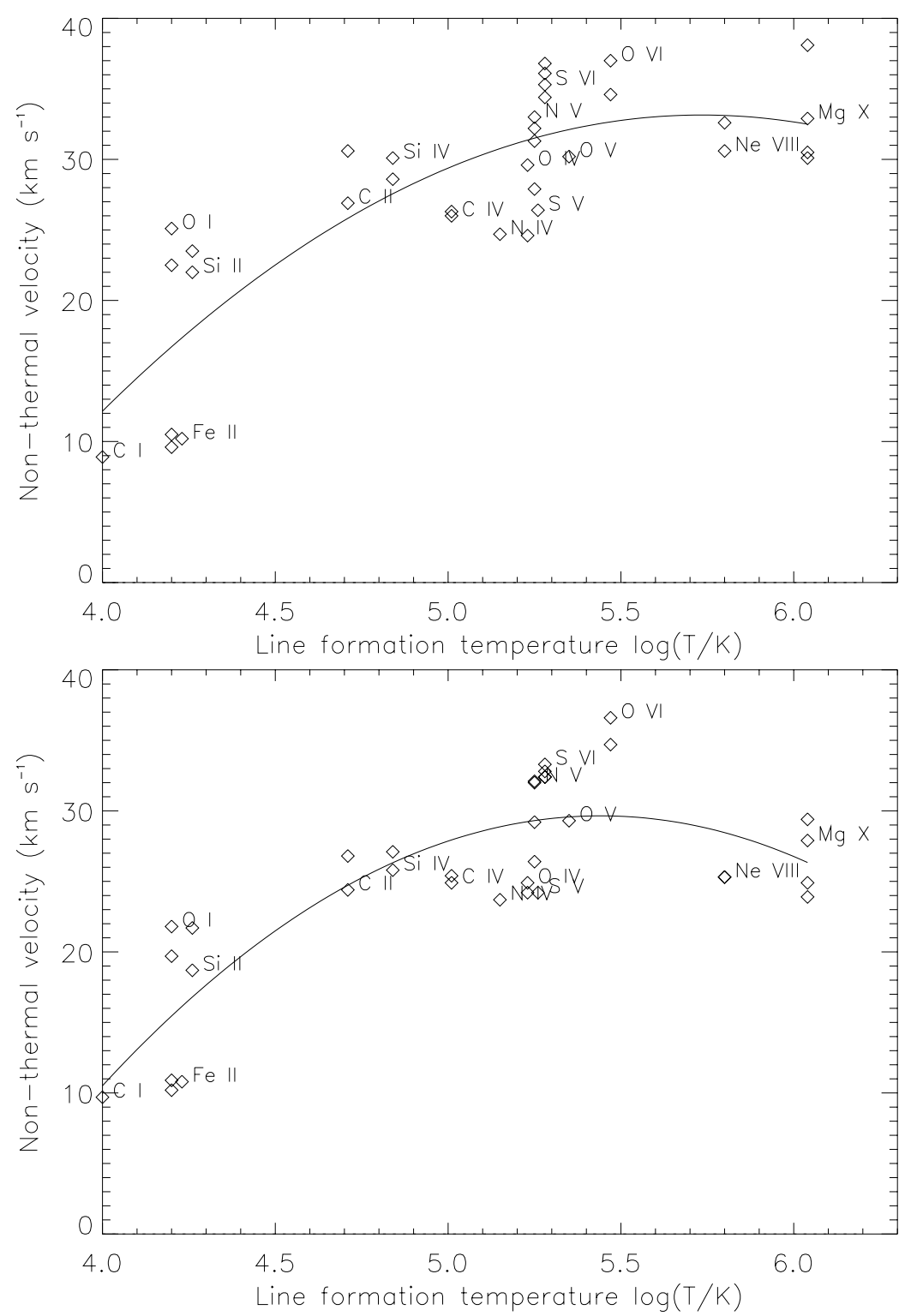

Figure 6.16: Average line width in various lines versus the formation temperature in $\mathrm{CH}$ and QS. (a) CH; (b) QS.

large variation in different holes. The possible reason will be discussed in the following section by comparison of the underlying magnetic fields in these holes.

Our comparison of the non-thermal velocity between the $\mathrm{CH}$ and QS regions confirms the results of some previous studies, in which it was found that there is a slightly larger Doppler width in $\mathrm{CH}$ than in QS regions (Wilhelm et al., 2000; Dere et al., 1989b; Lemaire et al., 1999; Stucki et al., 1999). From all these studies, a conclusion may be made that the difference of the measured line width in the two regions is small for the lines formed below the upper transition region. 
Furthermore, the non-thermal velocity deduced from the $\mathrm{Ne}$ VIII and $\mathrm{Mg} \mathrm{X}$ lines in the $\mathrm{CH}$ region are not inconsistent with that measured above the limb in polar holes. Doschek et al. (2001) found that the Ne VIII line width implies a non-thermal velocity of $35 \mathrm{~km} \mathrm{~s}^{-1}$ at the limb, while the $\mathrm{Mg} \mathrm{X}$ line has a value of $39 \mathrm{~km} \mathrm{~s}^{-1}$ at about $30^{\prime \prime}$ above the limb.

\subsection{Plasma parameters deduced from coronal lines and relation to the underlying magnetic field}
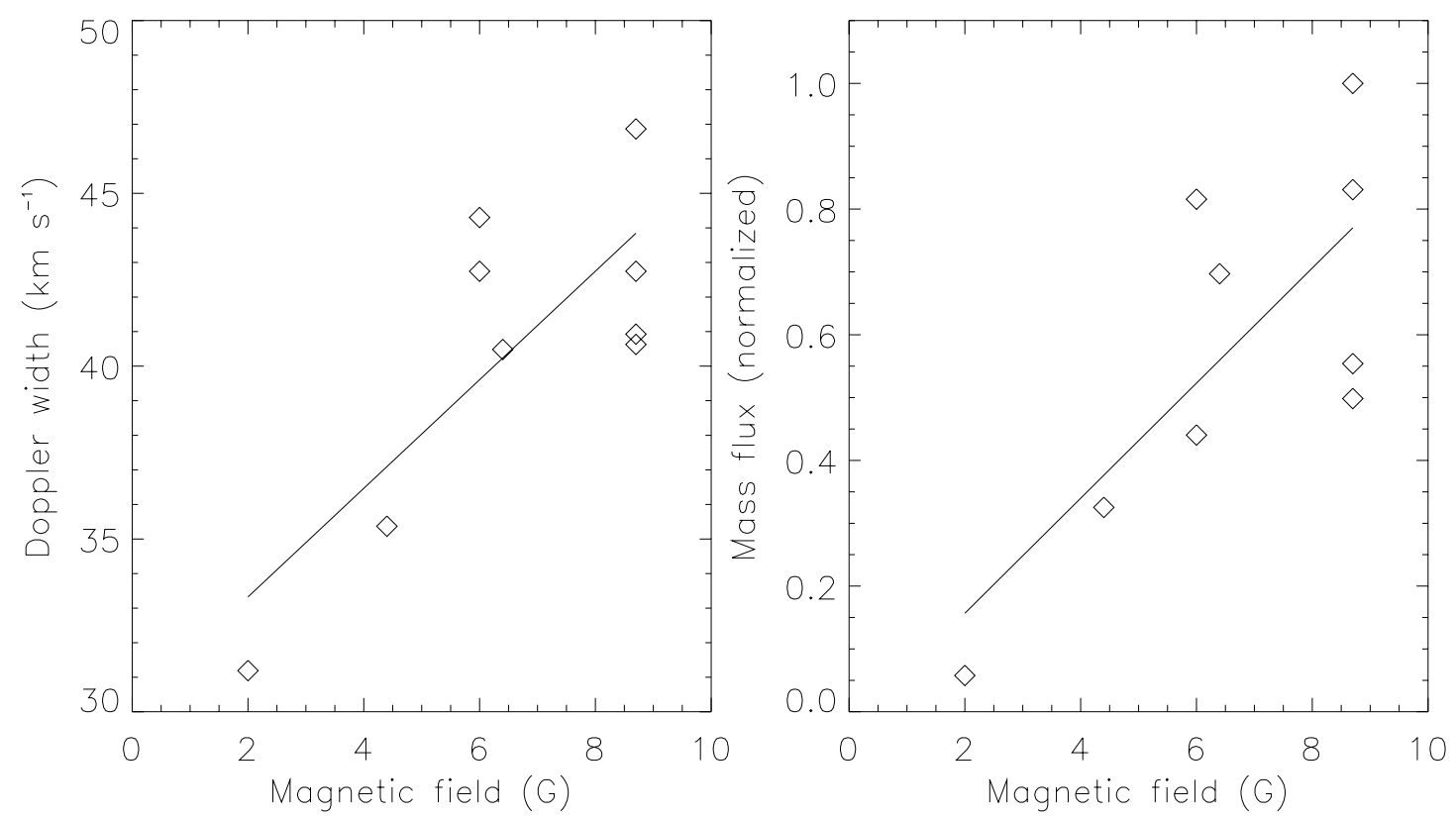

Figure 6.17: Left: Line width of the $M g X$ line versus the magnetic field observed in CHs. Right: Derived mass flux versus the magnetic field.

In Table 6.1, line parameters of the $\mathrm{Mg} \mathrm{X}$ line have been measured in five coronal holes. Among them, two were observed during solar minimum and three at solar maximum. The results show that the plasma properties inferred by the $\mathrm{Mg}^{9+}$ ion seem to be different in these five holes. By inspection of the magnetic field data observed by NSO/KP, the average field flux density exhibits a different value in these holes. Motivated by this notion, we search for a possible relationship between the plasma parameters deduced from coronal lines and the underlying magnetic field.

In this study, all the plasma parameters are deduced from the $\mathrm{Mg}^{9+}$ ion, which is ionized at a temperature of around 1.1 MK. At this coronal temperature, the whole corona appears to be fairly homogeneous at a scale large compared with the network. The $\mathrm{Mg} \mathrm{X}$ emission pattern also indicates a more uniform coronal magnetic field there, although we can not measure it directly. An reasonable assumption may be that the measured plasma parameters represent the average properties in the coronal hole at a height where the $\mathrm{Mg}^{9+}$ ion 
has its maximal emission. These properties may be determined directly by the underlying photospheric magnetic field which can be measured by remote sensing observations. Considering that the large-scale magnetic field in the coronal hole is unipolar, we calculate only the net magnetic field flux density (signed) inside the hole, and assume that bipolar structures are locally closed (without large-scale bipolar structures reaching into the corona).

In the left panel of Figure 6.17, the line width of the $\mathrm{Mg}$ X line versus the net flux density of the magnetic field is plotted for different coronal holes. Instead of averaging the values, which are deduced from different datasets obtained in the same hole by SUMER, we present them in a scatter plot shown in the figure. The solid line represents a linear fit to the data, which is just used to indicate the trend. In the figure, a positive correlation between the magnetic field and line width $v_{1 / e}$ can be found. The line width shows a clear trend and increases with the increase of the underlying magnetic field. Such a relation between the two parameter seems to be an important constraint on theory, and may be a key to understand the heating process of the coronal hole.

Using the same method as discussed in Section 5.6, we investigate a possible relationship between the magnetic field and the outward mass flux. The proton (main component of the solar wind) density can be roughly represented by the electron density. In principle, the electron density ratio between different regions can be estimated by the line intensity ratio, if we simply assume that the emission volume (having the same bottom area) is of the same size for the $\mathrm{Mg} \mathrm{X}$ line, and that the electron temperature is same in these regions. A further assumption is that the deduced Doppler shift of the $\mathrm{Mg} X$ line represents the real outflow velocity of the $\mathrm{Mg}^{9+}$ ions which are assumed to be markers of the proton flow.

In the right panel of Figure 6.17, we plot the quantity of $v \sqrt{I}$ (with $I \sim n_{e}^{2}$ ) indicating the outward mass flux versus the magnetic field flux density for different coronal holes. Again, the linear fit (solid) is just used to show the trend. A clear positive correlation between the two quantities can be found from this figure. If this estimation of the mass flux ratio between different holes is reliable, it would place an observational constraint on the mass flux. Our findings indicate that the mass flux of the nascent fast solar wind may be directly associated with the net flux density of the magnetic field in the hole.

One should bear in mind, however, that the assumptions we have made above may be violated in the case that different coronal holes are involved. There may be two reasons: First, we don't know exactly the emission volume; it may vary between different regions. Second, different holes may have a different electron temperature, and therefore the $\mathrm{Mg}$ $\mathrm{X}$ emissivity may vary, since it depends on the electron temperature. For example, if one hole has an electron temperature equal to the line formation temperature, and another hole a lower one, then the total emissivity of the line will be lower in the latter hole, even if the electron density and emission volume are same for the two regions. This is possible in coronal holes because their electron temperature is often lower than $1 \mathrm{MK}$ (see, e.g., Marsch, 1999), which is lower than the formation temperature of the $\mathrm{Mg}$ X line. A more reliable approach would be to measure the electron density directly, which will be done for $\mathrm{CH} 1$ and $\mathrm{CH} 2$ (see the next chapter). In that study, we will show that the mass flux 
is indeed larger in $\mathrm{CH} 1$ than in $\mathrm{CH} 2$ by a factor of about 2.5. Simply estimated from the line ratio of the $\mathrm{Mg}$ X line shown in the right panel of Figure 6.17, this factor is about 5.5. Unfortunately, because the parameters for the other three $\mathrm{CHs}$ were measured by using detector A of SUMER, it is impossible to determine directly the coronal electron density. Therefore, the empirical relations established here between the net magnetic field strength and plasma parameters need be confirmed in future work.

\subsection{Summary and discussion}

In this chapter, a statistical and quantitative study has been carried out with SUMER data obtained in five ECHs and a QS region located on the disk center. Line parameters deduced from various lines in both $\mathrm{CH}$ and QS regions have been used to investigate the plasma properties in these regions. Moreover, a relationship between deduced line parameters of the coronal line of $\mathrm{Mg} \mathrm{X}$ and the underlying magnetic field observed by $\mathrm{NSO} / \mathrm{KP}$ has been investigated. The main results are summarized as follows:

- From the histogram plots of the line parameters, the intensity distribution shows that a slight difference between CHs and the QS region has already presented from the upper chromosphere. A prominent difference can be seen in the transition region lines, in particular for the Li-like ions and helium. The apparent higher counts in the high-intensity tail for the Li-like ions and helium are consistent with the morphologic results that the loop-like structures of these lines are more extended into the cell interiors in CHs than in the QS region.

- The average intensity is lower for transition region and coronal lines in CHs than in the QS region. The intensity ratio between $\mathrm{CH}$ and QS regions generally decreases with the formation temperature, in agreement with previous studies. Such a decrease of the intensity ratio is found to be more clearly for the helium and Li-like ions. There is a weak evidence that the lines formed in the upper chromosphere are slightly stronger in the $\mathrm{CH}$ region.

- The distribution of the Doppler shift exhibits a more spread profile for the O VI, Ne VIII and $\mathrm{Mg}$ X lines with a high formation temperature, while for other cooler lines it shows a same trend, but less pronounced.

- Except for the Ne VIII and Mg X lines, all other lines appear on average red shifted in both $\mathrm{CH}$ and QS regions. Average red shifts of the transition region lines range mainly from $5 \mathrm{~km} \mathrm{~s}^{-1}$ to $10 \mathrm{~km} \mathrm{~s}^{-1}$ in the $\mathrm{CH}$ region, and $5 \mathrm{~km} \mathrm{~s}^{-1}$ to $15 \mathrm{~km} \mathrm{~s}^{-1}$ in the QS region. Small Doppler shifts are measured for the Ne VIII and $\mathrm{Mg} X$ lines in the QS region. On the other hand, both the Ne VIII and $\mathrm{Mg} X$ lines have average blue shift in $\mathrm{CHs}$. The temperature-dependence of average Doppler shifts in both $\mathrm{CH}$ and QS regions are consistent with previous results, i.e., the red shift increases first with increasing line formation temperature, then drops again at a temperature of about $0.3 \mathrm{MK}$ (in the $\mathrm{O} \mathrm{VI}$ line). Moreover, transition region lines have systematically smaller red shifts in $\mathrm{CHs}$ than 
in the QS region. The difference of the Doppler shift between $\mathrm{CH}$ and QS regions also show a temperature-dependent property.

- The distribution of the line width reveals a slightly wider value for all lines in $\mathrm{CHs}$, and to be more obvious for the Ne VIII and $\mathrm{Mg} X$ lines. By inspection of the line width deduced from averaged line profiles, it is found that the trend of non-thermal velocity varying with the temperature is similar as in the QS region as found by other authors. For the line width in $\mathrm{CHs}$, the variation trend of lines formed below the upper transition region is more or less similar as in the QS region. The difference between the two regions is small, with a larger value of around $1-2 \mathrm{~km} \mathrm{~s}^{-1}$ in CHs. An apparent difference arises for the Ne VIII and $\mathrm{Mg}$ X lines between $\mathrm{CH} 6$ and QS1, which were observed at a same solar active level. However, the line width of the $\mathrm{Mg} X$ line shows very large variation in different holes.

- It is found that an apparent relationship between the line parameters and the chromospheric network is present for the most measured lines. The chromospheric line of Si II reveals a very clear positive correlation between its Doppler shift, intensity and line width. The relatively red shifts and larger line widths are mainly corresponding to the network. For the He I line and transition region lines, such a relation becomes more complicated. In general, a larger blue or red shift usually has a larger line width and intensity, and also corresponds to a larger intensity of continuum, which implies that they mainly occur in the network. However, such a relationship is not clearly present for the C IV line, as was also found by other authors. In the Ne VIII line, there exists a slightly positive correlation between the Doppler shift and intensity. A bluer shift tends to have a lower intensity and a redder one corresponds to a larger intensity. For the line width, the tendency reveals to be inversely. Related to the continuum, the most counts with a larger blue shift have an averaged continuum intensity around the threshold defined as the boundary of network and cells. The result is consistent with the morphological results, which show that larger Doppler shifts come mainly from the network with a concentration of an unipolar magnetic field, but with a more areal extension compared with the area of the network defined in chromospheric lines. This also confirms that such a tendency deduced from the equatorial $\mathrm{CHs}$ is similar to the one in polar $\mathrm{CHs}$.

- There exists a clear positive correlation between Doppler shifts deduced from different lines that were observed simultaneously in the same spectral window. Moreover, the corresponding magnitudes of the Doppler shift vary with different lines formed at different layers, i.e., a larger value of the Doppler shift seen in the O VI line corresponds to a smaller one in the C II line and an even smaller one in the $\mathrm{H} \mathrm{I} \mathrm{L} \beta$ line. This statistical result nicely confirms our previous result obtained in the morphologic study.

- The line width deduced from the $\mathrm{Mg} \mathrm{X}$ line shows a clear trend to increase with increasing underlying magnetic field strength in CHs. Moreover, the quantity of $v \sqrt{I}$ (with $I \sim n_{e}^{2}$, which is used here as a measure of the coronal mass flux of the nascent fast solar wind, also reveals a clear positive correlation to the magnetic field strength. Such a relation between the line parameters and the underlying magnetic field seems to be an important constraint on theory, and may be a key to understand coronal heating and solar wind acceleration in coronal holes. 


\section{Chapter 7}

\section{Comparison of Coronal and in situ Observations}

\subsection{Introduction}

The correlation of the equatorial coronal holes and the high-speed streams observed at the Earth's orbit has been studied since the Skylab time (see, e.g., Krieger et al., 1973; Nolte et al. . 1976). It was found that the magnetic field in the coronal hole, which is rooted on the Sun's surface in the magnetic network and expands from there outward into the interplanetary space, has a rapidly diverging configuration (Zirker, 1977). In such largescale open fields, the coronal plasma can be quickly accelerated and become supersonic within several solar radii. As discussed in Chapter 2 and 5, it is suggested by theoretical work that the nascent fast solar wind can be accelerated to several tens of $\mathrm{km} \mathrm{s}^{-1}$ in the magnetic funnel (Marsch and Tu, 1997; Hackenberg et al., 2000; Vocks and Marsch, 2002; Li, 2002). The plasma velocity deduced by measuring the Doppler shift of the Ne VIII and $\mathrm{Mg} \mathrm{X}$ lines ranges from $3-12 \mathrm{~km} \mathrm{~s}^{-1}$ at the bottom of coronal holes (see Chapter 6). We believe that these blue shifts represent the real outflow velocity of the nascent fast solar wind, because (1) the Dopplergrams of these two lines show generally blue shifts in the coronal hole, and in particular, the $\mathrm{Mg} \mathrm{X}$ line has a more homogeneous velocity field; and (2) their Doppler shifts measured in the quiet Sun are rather small, which usually fall in the $0 \pm 3 \mathrm{~km} \mathrm{~s}^{-1}$ range, as expected, and do not show prominent large-scale outflow there.

The main purpose of the work described in this chapter is to study the relationship between the equatorial coronal holes and the related high-speed solar wind streams, by directly measuring the physical parameters inside the coronal holes and then comparing with those deduced from in situ observations of the solar wind. In details, we deduce the outflow velocity, electron density and photospheric magnetic field obtained by SUMER, MDI and NSO/KP and same parameters obtained by SWE and MFI onboard WIND at $1 \mathrm{AU}$, and discuss the conservation of the mass and magnetic flux and the expansion of solar wind stream tubes. This study is motivated by the following reasons: First, the photospheric magnetic fields can be measured reliably in low-latitude coronal holes. Second, the line-of-sight component of the velocity deduced from spectral lines can approximately represent the velocity of the real flow, if the Doppler shift can be interpreted as the bulk motion of the solar wind plasma. Third, high-speed solar wind streams produced by such CHs can be observed in the ecliptic plane near the Earth, where the wind parameters were routinely observed by some satellites. Fourth, during the 1996 "Whole Sun Month Campaign" (10 August to 8 September) properties of the "Elephant's trunk" hole have been intensively studied by using various instruments or through modelling work (see, reviews of Bromage et al., 2000; Gibson, 2001). For SUMER, the data obtained in the polar hole 
has been analyzed by Warren and Hassler (1999) and Doschek et al. (1998). However, SUMER observations of this "Elephant's trunk" hole at lower latitudes have not been reported.

The chapter is organized as follows: in the next section, we give a brief description of the selection of data and instruments; The relationship of the observed coronal holes and the high-speed solar wind streams are described in Section 3; Section 4 presents the diagnostic of the electron density in two holes by using SUMER data; Then in section 5, results from a detailed comparison of the coronal and in situ observations will be discussed; Finally, the summary and discussion are given in Section 6.

\subsection{Data selection}

The data were obtained in two equatorial coronal holes $(\mathrm{CH} 1$ and $\mathrm{CH} 2$ as listed in Table 4.1) by SUMER during the solar minimum in the second half of 1996. The "reference spectrum" mode and slit 2 with the size of $1^{\prime \prime} \times 300$ "were used. The data were telemetered from SUMER's detector B. This provides an opportunity to use coronal lines such as the Mg IX lines, which can not be recorded in detector A in the first order, to measure the electron density in the corona. Moreover, simultaneous data obtained by four other instruments onboard the spacecraft SOHO and WIND as well as the photospheric magnetic field from NSO/Kitt Peak were also completely available and thus can be used. The instruments and their measurement items are listed in Table 7.1

Table 7.1: Instruments and their measurement items: comparison of coronal and in situ observations

\begin{tabular}{|c|c|}
\hline Instruments & Measurements \\
\hline SUMER & intensity, Doppler shift, line width and electron density \\
\hline EIT & 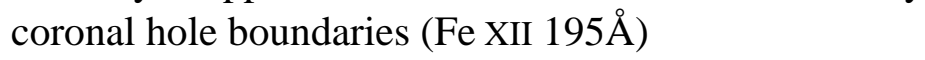 \\
\hline MDI & photospheric magnetic field \\
\hline $\mathrm{NSO} / \mathrm{KP}$ & photospheric magnetic field \\
\hline SWE & solar wind speed, density and temperature at $1 \mathrm{AU}$ \\
\hline MFI & interplanetary magnetic field at $1 \mathrm{AU}$ \\
\hline
\end{tabular}

\subsection{Equatorial coronal holes and high-speed solar wind streams}

During the solar minimum in the second half of 1996, a large equatorial coronal hole (CH1), which extended from the north pole and was connected with an active region at the latitude of about $20^{\circ} \mathrm{S}$, was one of the most prominent features seen on the solar disk. This 


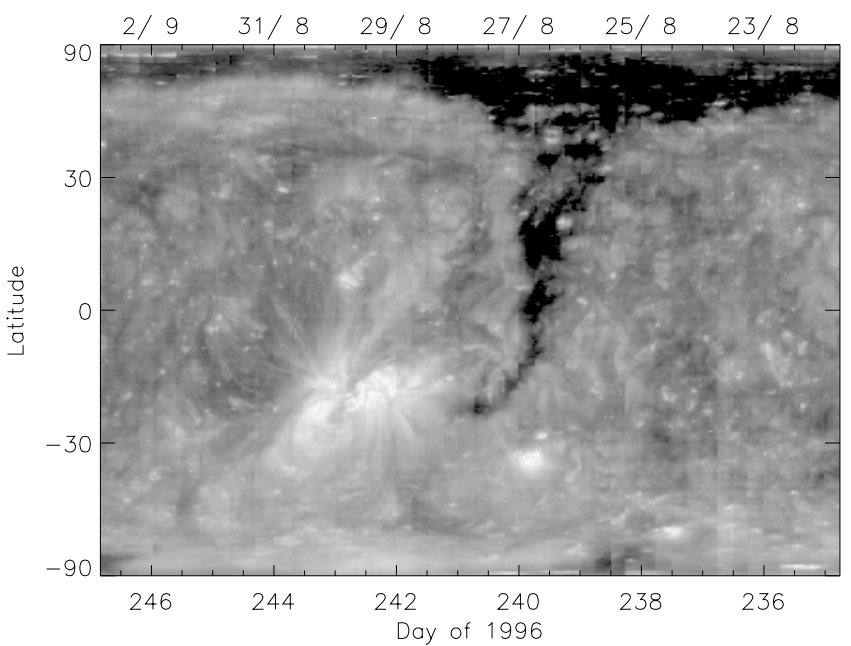

Figure 7.1: EIT meridian map during the period from 22 August to 3 September, 1996, showing the recurrent "Elephant's Trunk" coronal hole.

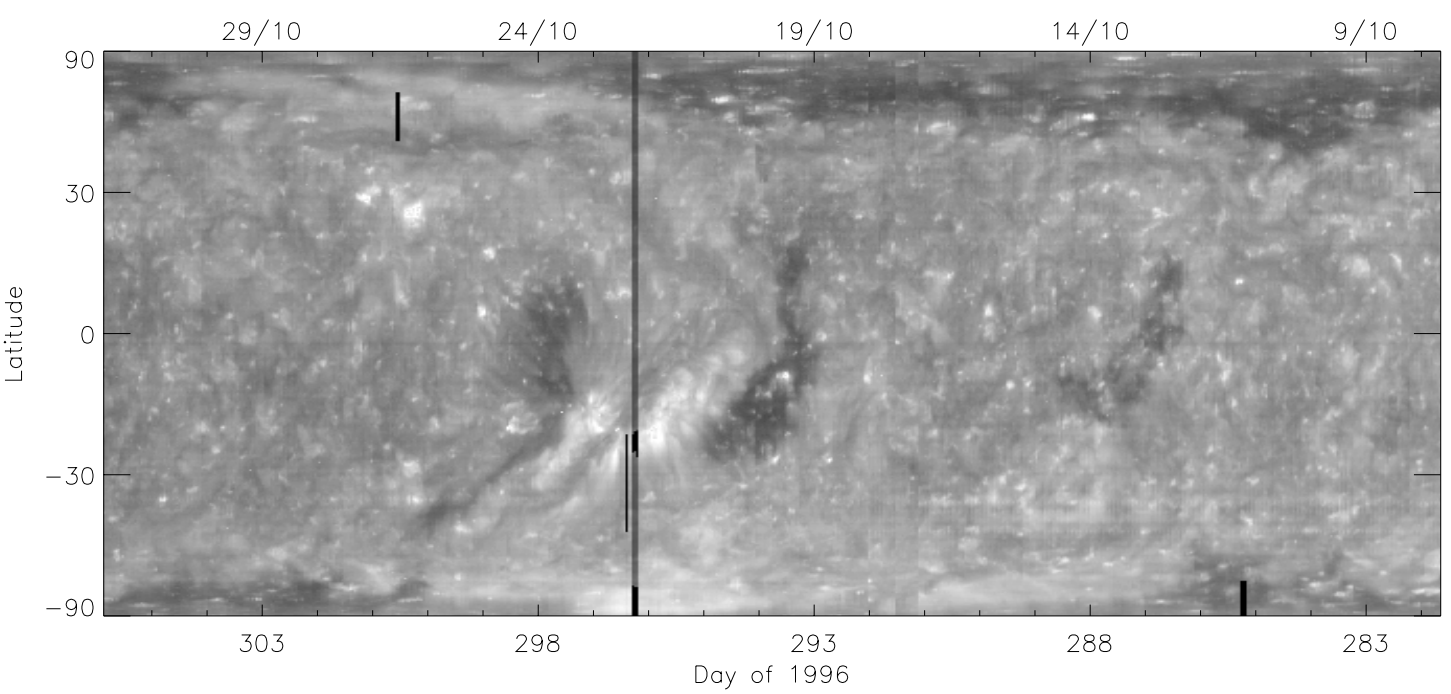

Figure 7.2: EIT meridian map during the period from 8 October to 1 November, 1996, showing three equatorial coronal holes.

"Elephant's trunk" hole can be seen in the EIT meridian map as dark region in Figure 7.1. This meridian map was observed by EIT in the Fe XII $195 \AA$ channel during the time from 22 August to 3 September, 1996, as a part of the Carrington rotation (CR) 1913. The main structure of this "Elephant's trunk" persisted for several months and could still be clearly seen in the center of the EIT meridian map from 8 October until 1 November (part of CR 1914 and 1915), but then was disconnected from the polar region (see Figure 7.2). On each side of this hole, two isolated coronal holes $(\mathrm{CH} 2$ and $\mathrm{CH} 4 \mathrm{4})$ can be seen in this figure. Checking the photospheric magnetic field observed by MDI and NSO/KP, it can be inferred that $\mathrm{CH}_{1}, \mathrm{CH} 2$ and $\mathrm{CH} 3$ have a positive polarity of the average net magnetic flux density (direction away from the Sun), while $\mathrm{CH} 4$ has a negative one. 


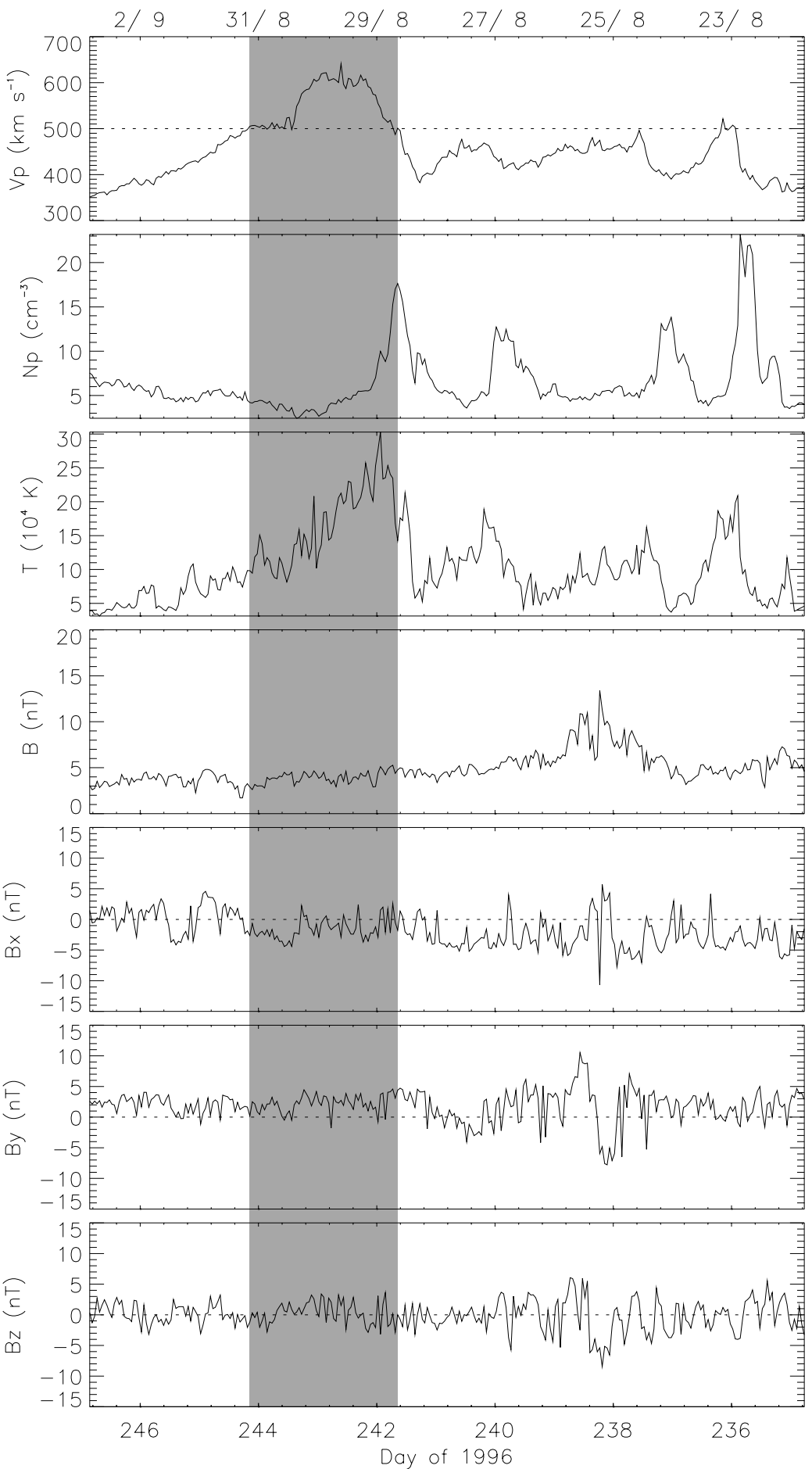

Figure 7.3: Solar wind parameters observed by SWE and MFI on WIND at 1 AU during the same period as Figure 7.1 showing a related high-speed stream. Note the displacement of the fast stream with respect to the $\mathrm{CH}$ in time, which is due to the travel time (3-5 days) of the stream from the corona to $1 \mathrm{AU}$. 


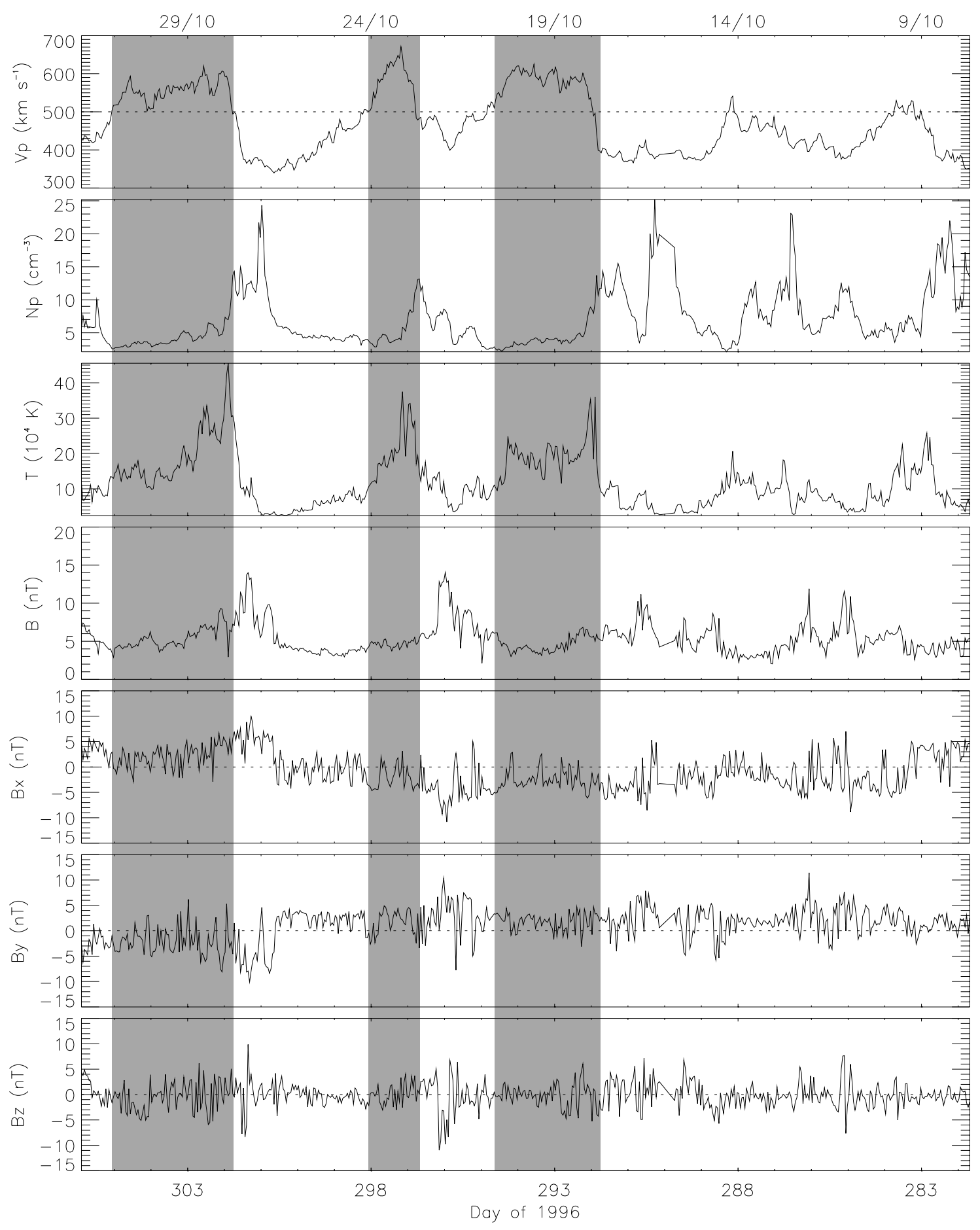

Figure 7.4: Solar wind parameters showing three related high-speed streams observed by SWE and MFI on WIND at 1AU during the same period as Figure 7.2, which shows the three associated source $\mathrm{CHs}$. 
The four $\mathrm{CHs}$ and their related high-speed streams are shown in Figures 7.3 and 7.4. In the two figures, we plot hourly-averaged plasma parameters of the solar wind (proton velocity, density, temperature) measured by SWE/WIND and the interplanetary magnetic field (IMF) (magnitude and three vector components in GSE coordinates) observed by MFI/WIND at 1AU during the same period as in Figures 7.1 and 7.2. The data were obtained when the WIND satellite was in GSE coordinates located at $\mathrm{X}_{G S E}=30 \mathbf{R}_{E} \sim$ $130 \mathrm{R}_{E}\left(\mathrm{X}_{G S E}\right.$ pointing to the Sun, $\left.\mathrm{R}_{E} \sim 6400 \mathrm{~km}\right)$ and $\mathrm{Y}_{G S E}=-70 \mathrm{R}_{E} \sim 10 \mathrm{R}_{E}\left(\mathrm{Y}_{G S E}\right.$ pointing to the south direction). Therefore, its orbit was nearly in the ecliptic plane.

High-speed solar wind streams related to these coronal holes can be readily distinguished in Figures 7.3 and 7.4. They have typical properties, i.e., high proton velocities of above $500 \mathrm{~km} \mathrm{~s}^{-1}$, low proton densities of about $3-5 \mathrm{~cm}^{-3}$ and high proton temperatures of around $2 \times 10^{5} \mathrm{~K}$ (deduced from the most probable proton velocity). It should be noted that the time delays of the high-speed streams, compared with the time when their corresponding source regions occurred, are due to the travel time (3-5 days) of the wind from the corona to $1 \mathrm{AU}$. Moreover, compared with the typical fast solar wind, having an average velocity of $750 \mathrm{~km} \mathrm{~s}^{-1}$ as observed in the polar hole by Ulysses (Woch et al. 1997; McComas et al., 2000), the high-speed streams produced by these coronal holes had lower velocities ranging between 550 and $650 \mathrm{~km} \mathrm{~s}^{-1}$, as also found by other authors (see, Gibson, 2001, and references therein).

From inspection of the magnetic field data, it can be found that the streams corresponding to $\mathrm{CH} 1, \mathrm{CH} 2$ and $\mathrm{CH} 3$ have a positive polarity of the interplanetary magnetic field, while the stream corresponding to $\mathrm{CH} 4$ has a negative one. They are consistent with the polarities of the photospheric magnetic field observed in these coronal holes.

\subsection{Diagnostic of electron density in coronal holes}

\subsubsection{Line intensity ratios}

As discussed in 3.4.3, the intensity ratio of an allowed emission line and a forbidden or intersystem emission line can, due to its density-sensitivity, be used to determine the electron density, making the assumption of equal electron and ion temperatures. With the SUMER spectrometer, measurements of electron density take full advantage of the high spectral resolution and wide spectral range of wavelengths. In particular, some line pairs are obtained from the same spectral window of SUMER, which ensures that the measured emission is really from the same plasma regime. In order to study the electron pressure of the transition region, measurements of electron densities in various regions have been carried out by using several density-sensitive line pairs (Si III, O V, Ne VII, Mg VIII, and Mg IX) observed by SUMER (see, e.g., Doschek et al., 1998; Warren and Hassler, 1999).

For the study of the equatorial coronal holes, such measurements become more difficult due to the reduced emission in holes and the blends of cooler lines on the disk. Line profiles of useful line pairs suggested by some authors (Warren and Hassler, 1999; 
Doschek et al., 1998; Wilhelm et al., 1995; Laming et al., 1997; Mariska, 1992) have been checked carefully. We found that only the line pairs Si III, O V and Mg IX can be used for our study. Fortunately, the formation temperatures of the Si III, O V and Mg IX line are around $0.05 \mathrm{MK}, 0.25 \mathrm{MK}$ and $1 \mathrm{MK}$, respectively, and can thus represent three regions of the solar atmosphere ranging from the lower transition region to the coronal base. The theoretical intensity ratio versus the electron density of these three line pairs are shown in Figure 7.5. The three curves were calculated by using the CHIANTI atomic database and software (Dere et al., 1997). It should be mentioned that the line ratios of the Si III and O V pair have a pronounced dependence on the electron temperature (see dotted and dashed lines in Figure 7.5). Detailed comments on these line pairs can be found in Mariska (1992), Warren and Hassler (1999) and Doschek et al. (1998).

\subsubsection{Line profiles deduced from SUMER data}

The $\mathrm{CH} 1$ and $\mathrm{CH} 2$ including the positions of the SUMER slit are shown in Figures 7.6 and 7.7. Because the coronal lines are quite weak in these $\mathrm{CHs}$, we have binned the data along the slit in the $\mathrm{Y}$ direction, thereby selecting areas obtained from homogeneous dark parts of the holes, from $190^{\prime \prime}$ to $335^{\prime \prime}$ for $\mathrm{CH} 1$ (Figure 7.6) and from $-60^{\prime \prime}$ to $50^{\prime \prime}$ for $\mathrm{CH} 2$ (Figure 7.7), in order to avoid inclusion of the bright structures. There two reasons for excluding the bright structures. First, as discussed in Chapter 5, these structures in coronal holes have a high radiance but contribute only a small part of the mass flux to the solar wind. Second, inclusion of such structures, which occurred mainly in regions having magnetic features with mixed polarities and bright emissions, will lead to smearing of the information gained from the dark parts of the holes. These dark areas have been confirmed to produce plasma with a high outward velocity and to be the main source of the fast solar wind (see Chapter 5).

The spatially averaged profiles of these lines together with the fitted ones are shown in Figure 7.8, which was deduced from the dataset obtained in $\mathrm{CH} 2$. In the most cases, multiGaussian fitting is used, because of the blends with other lines. Usually, one line of each line pair is strong enough to be fitted reliably (see Figure 7.8 (a), (c), (f) for lines Si III at $1296 \AA, \mathrm{O}$ V at $758 \AA$ and Mg IX at $706 \AA$, respectively), while another one is quite weak (see Figure 7.8 (b), (d) for lines Si III at $1301 \AA$ and $\mathrm{O} V$ at $761 \AA$ ) or blended by the neighbouring line (see Figure 7.8 (e) for the Mg IX line at $694 \AA$, which is near the Na IX line at $694.13 \AA$ ). For each line pair, we therefore fit first the strong one and obtain the line width, and then fit the weak one by assuming that it has the same line width as the strong one. This procedure can reduce the number of fit parameters and make the fitting more reliable. It should also be mentioned that, although the Si III line (1301 $\AA$ ) is very weak, it can still be fitted nicely owning to the smooth background around this line.

Warren and Hassler (1999) have mentioned that the line widths deduced from their datasets taken between 0232 UTC and 0830 UTC on 27 August, 1996 were systematically wider by about $20 \%$ than they were in the following observations, because the SUMER's detector B was used before it was optimized for focus. The datasets selected 


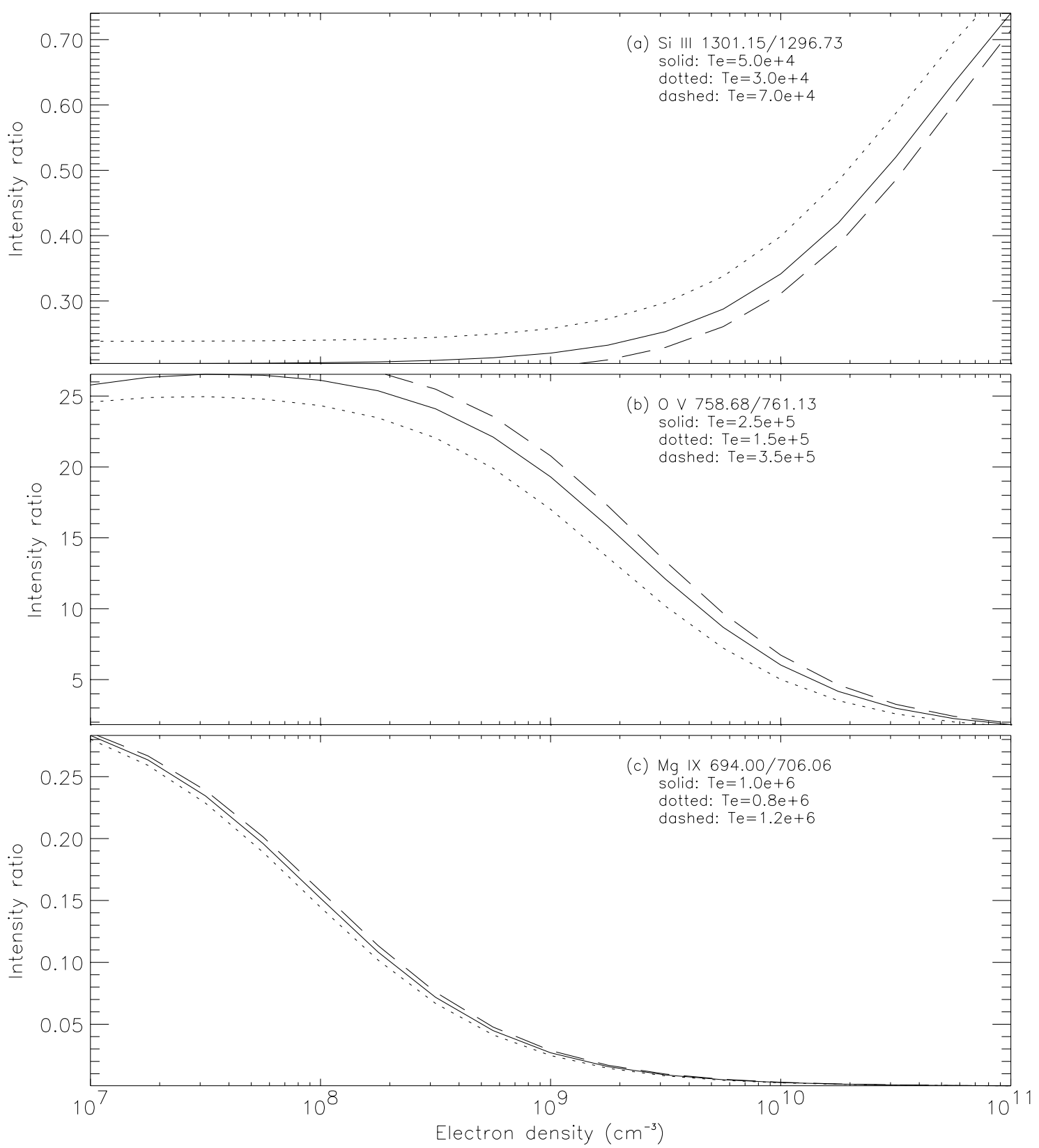

Figure 7.5: Theoretical line intensity ratios versus electron density for different electron temperatures. The ratios are calculated by means of the CHIANTI atomic database and software. The solid lines correspond to the temperatures of ionization equilibrium. (a) Si III; (b) $\mathrm{OV}$; and (c) $\mathrm{Mg}$ IX. 

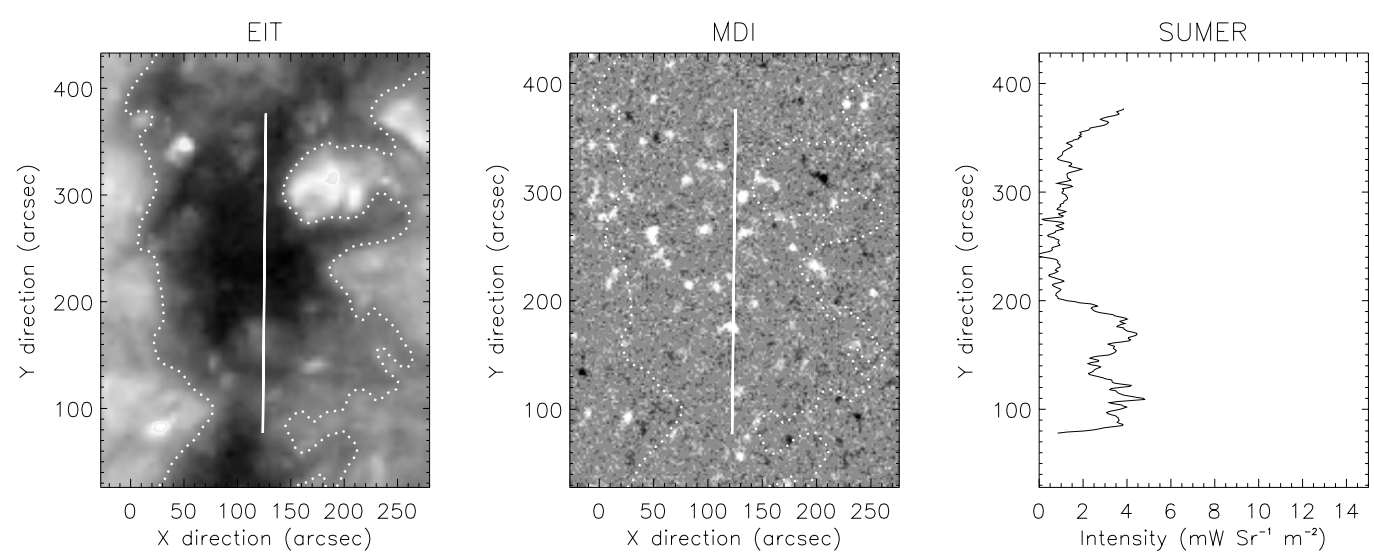

Figure 7.6: Left: EIT map on 27 August, 1996, showing part of the coronal hole "Elephant's Trunk". Middle: MDI magnetogram. Right: Integrated line intensity of the Mg IX line (706 $\AA$ ) along the slit of SUMER. Note the coronal hole boundary indicated by a dotted line and the SUMER slit position indicated by a solid line in the EIT and MDI images.
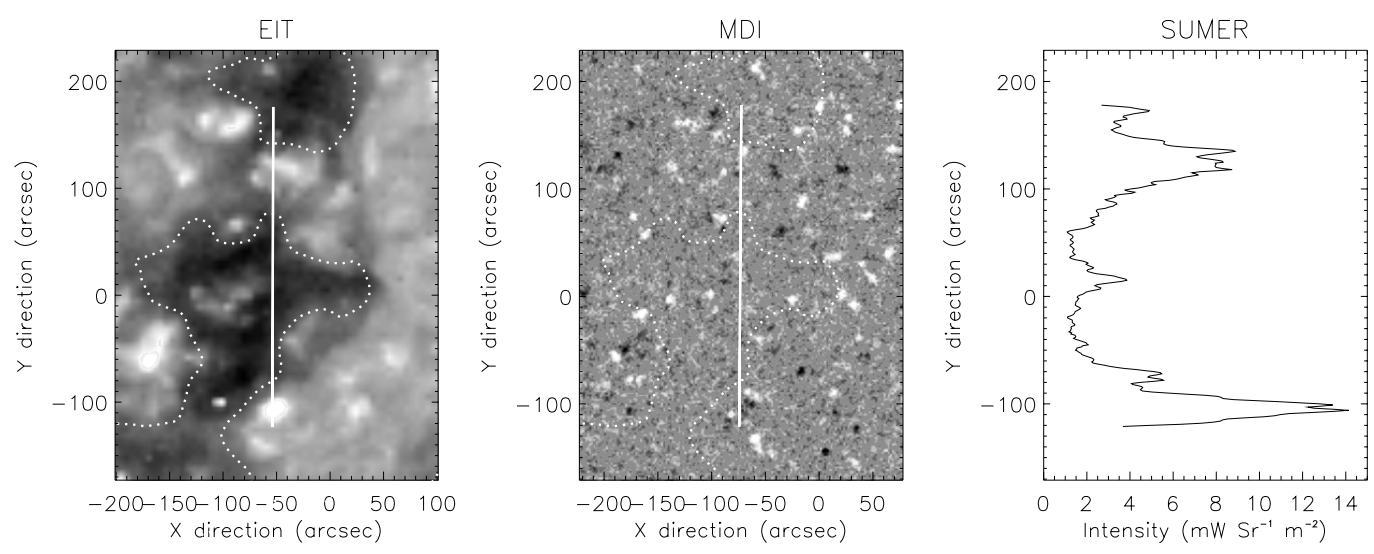

Figure 7.7: The same as Fig. 7.6 but for the coronal hole observed on 12 October, 1996.

here were taken between 1125 UTC and 1635 UTC. A careful check of our datasets has been carried out by comparing the line widths of cooler lines with those observed later in $\mathrm{CH} 2$ on 12 October, 1996 . Only a $2 \%$ difference in the line width has been found for the S I line (1300.9 ̊). Thus, the datasets used here have no such effects.

\subsubsection{Electron densities deduced from SUMER data}

After having observed the line intensity ratios of three line pairs, the theoretical line intensity ratios shown in Figure 7.5 are used to determine the electron densities in $\mathrm{CH} 1$ and $\mathrm{CH} 2$. $\mathrm{CH} 1$ was also observed by $\mathrm{CDS} / \mathrm{SOHO}$ at the same time. $\mathrm{CDS}$ covered an area ranging from $-50^{\prime \prime}$ to $200^{\prime \prime}$ in the solar Y-direction (Del Zanna and Bromage, 1999), while the SUMER slit covered it from $80^{\prime \prime}$ to $380^{\prime \prime}$. From the EIT map and also the intensity of the Mg IX line shown in Figures 7.6, the coronal hole appears brighter below 190" in Y- 

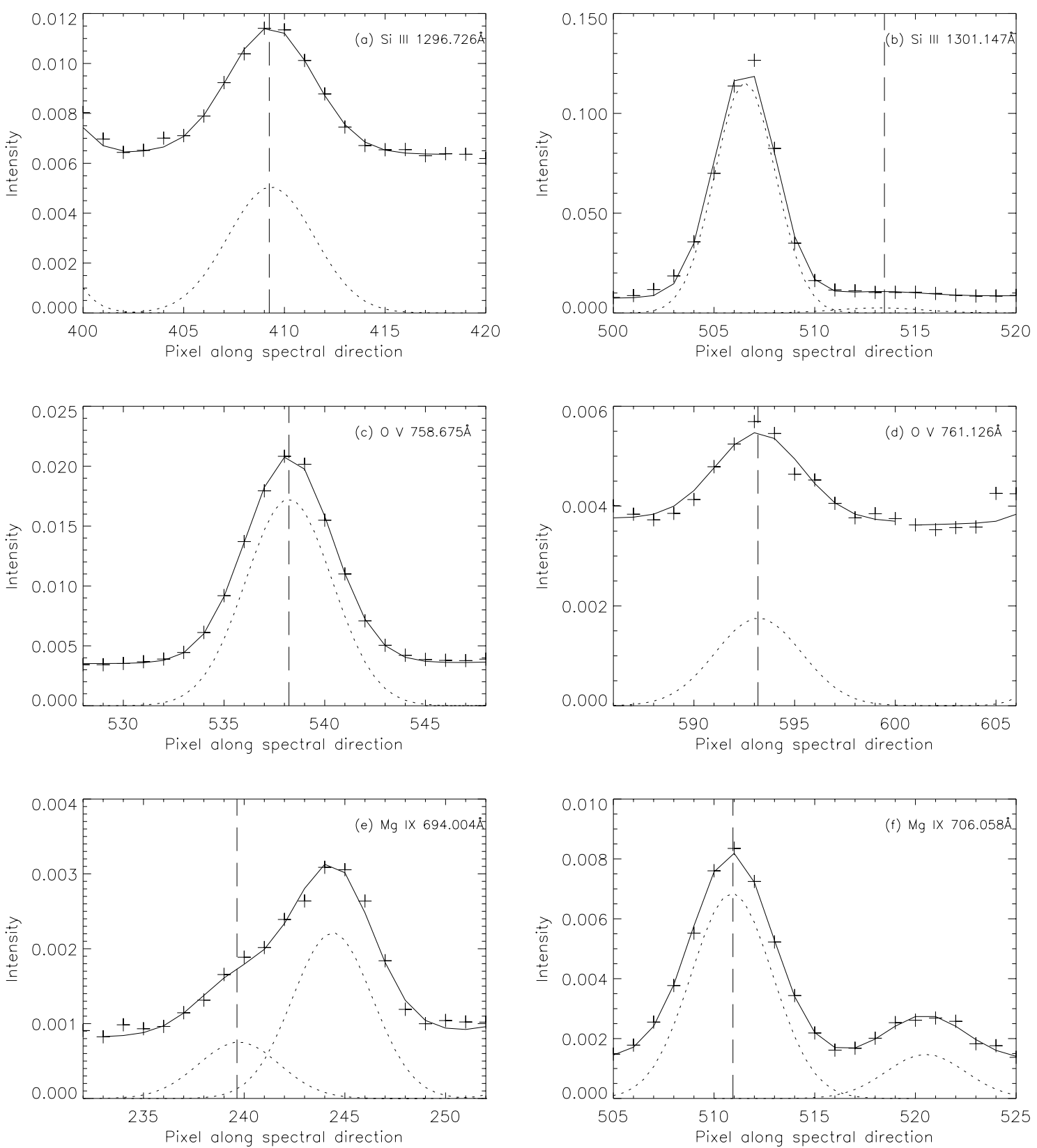

Figure 7.8: Line profiles of line pairs Si III, $O V$ and $M g$ IX used to measure electron densities. The " + " represents the data points from observations. Dashed lines indicate the positions of these lines. Dotted lines are line profiles fitted with the Gaussian shape, while the solid lines represent the profiles obtained by background plus Gaussian fitting. Note that the units of the intensity is $W$ $s r^{-1} \mathrm{~m}^{-2} \AA^{-1}$. 
direction. The average electron density along the slit below $335^{\prime \prime}$ in $\mathrm{CH} 1$ (combined with both dark and bright area) has been measured to be about $2 \pm 0.5 \times 10^{8} \mathrm{~cm}^{-3}$, which is consistent with the result obtained by Del Zanna and Bromage (1999) using the line pair Si IX (350 $\AA / 342 \AA$ ), which has a similar formation temperature as the line pair of Mg IX. With SUMER data obtained $10^{\prime \prime}-20^{\prime \prime}$ above the limb, Warren and Hassler (1999) used this line pair of Mg IX and measured the electron density to be $1.7 \times 10^{8} \mathrm{~cm}^{-3}$.

Integrated line intensities, intensity ratios and deduced electron densities together with the uncertainties measured in the dark regions of $\mathrm{CH} 1$ and $\mathrm{CH} 2$ are listed in Table 7.2 . The uncertainties are determined only from the fitting errors of integrated line intensities and widths. For the two transition region lines ( $\mathrm{Si}$ III and O V), the differences between the two coronal holes is marginal when compared with the pronounced uncertainties of the measurements. The results are comparable with those obtained by Doschek et al. (1998) in the quiet Sun and coronal holes on the disk. In the Mg IX line, the electron density measured in $\mathrm{CH} 1$ is only about $8 \times 10^{7} \mathrm{~cm}^{-3}$, which is a factor of 2 lower than that measured in the same hole, but including all structures in the hole. The measurement uncertainties of this line pair are relatively small. In fact, a lower electron density has been also obtained by Young and Esser (1999) using CDS/SOHO data observed in dark regions of the polar hole. These indicate that inhomogeneities would indeed result in a multi-density structures of the plasma (Doschek, 1984). The density measured in $\mathrm{CH} 1$ is also lower by a factor of 2 than in $\mathrm{CH} 2$. We suggest that this may be due to different conditions prevailing in these two holes, which will be discussed in the following section.

Moreover, the electron pressure can be inferred from the measured electron density at different temperatures. In the transition region, the electron pressure can be estimated to be about $1-1.5 \times 10^{15} \mathrm{~K} \mathrm{~cm}^{-3}\left(\mathrm{p}_{e}=\mathrm{N}_{e} \mathrm{~T}_{e}\right)$ from the Si III and $\mathrm{O} \mathrm{V}$ lines, and infers to a constant electron pressure of the transition region for each hole. From the Mg IX line, the electron pressure at the coronal base is about $1 \times 10^{14} \mathrm{~K} \mathrm{~cm}^{-3}$ in $\mathrm{CH} 1$ and $2 \times 10^{14}$ $\mathrm{K} \mathrm{cm}^{-3}$ in $\mathrm{CH} 2$. The difference in electron pressure between the transition region and the coronal base is large ( $7-10$ times higher in the transition region). Hence, the result demonstrates that there exist large thermal pressure gradients between the two layers. This thermal pressure gradient may drive the initial outflows of the fast solar wind in coronal funnels.

Table 7.2: Electron densities deduced from SUMER data

\begin{tabular}{|c|c|c|c|c|c|c|}
\hline Ion & $\begin{array}{l}\lambda \\
(\AA)\end{array}$ & $\begin{array}{l}\mathrm{T}_{e} \\
(\mathrm{MK})\end{array}$ & Region & 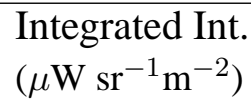 & Ratio & $\begin{array}{l}\mathrm{N}_{e} \\
\left(\mathrm{~cm}^{-3}\right)\end{array}$ \\
\hline \multirow[t]{2}{*}{ Si III } & $1301.15 /$ & 0.05 & $\mathrm{CH} 1$ & $794 / 1822$ & $0.44 \pm 20 \%$ & $2.0_{-0.9}^{+1.3} \times 10^{10}$ \\
\hline & 1296.73 & & $\mathrm{CH} 2$ & $583 / 1153$ & $0.50 \pm 20 \%$ & $2.8_{-1.3}^{+2.0} \times 10^{10}$ \\
\hline \multirow[t]{2}{*}{$\mathrm{OV}$} & $758.68 /$ & 0.25 & $\mathrm{CH} 1$ & $4430 / 418$ & $10.60 \pm 18 \%$ & $4.0_{-1.0}^{+1.6} \times 10^{9}$ \\
\hline & 761.13 & & $\mathrm{CH} 2$ & $4137 / 419$ & $9.87 \pm 7 \%$ & $4.6_{-0.5}^{+0.6} \times 10^{9}$ \\
\hline \multirow[t]{2}{*}{ Mg IX } & $694.00 /$ & 1.0 & $\mathrm{CH}$ & $116 / 685$ & $0.17 \pm 17 \%$ & $0.8_{-0.3}^{+0.4} \times 10^{8}$ \\
\hline & 706.06 & & $\mathrm{CH} 2$ & $169 / 1538$ & $0.11 \pm 15 \%$ & $1.8_{-0.4}^{+0.4} \times 10^{8}$ \\
\hline
\end{tabular}




\subsection{Comparison of coronal and in situ observations}

The measurement of the Doppler shift has been discussed in Chapter 4 and 6 . In this study, the Mg X line (624.968 $\AA, T_{e}=1.1 \mathrm{MK}$ ) observed in 2nd order is used to deduce the outflow speed at the coronal base. The formation temperature is near that of the $\mathrm{Mg}$ IX lines, which are used to measure the electron density. For wavelength calibration we used cool lines of C I. Chae et al. (1998c) have estimated that C I lines have only a small average red shift of about $1.5 \mathrm{~km} \mathrm{~s}^{-1}$. This value has been subtracted from the average Doppler shift of the $\mathrm{Mg} \mathrm{X}$ line. By assuming that the velocity of the $\mathrm{Mg}^{9+}$ ion represents the bulk velocity of the protons, the particle flux of the solar wind at the coronal base can be estimated to be $6.3 \times 10^{13} \mathrm{~cm}^{-2} \mathrm{~s}^{-1}$ in $\mathrm{CH} 1$ and $2.5 \times 10^{13} \mathrm{~cm}^{-2} \mathrm{~s}^{-1}$ in $\mathrm{CH} 2$. All the values are list in Table 7.3 ,

MDI (Michelson Doppler Imager) full-disk magnetograms (sampled at a rate of 15/day) are used to estimate the average photospheric magnetic field across those areas that spatially coincide with the ones selected to deduce the outflow speed and electron density by using SUMER data (Figures 7.1 and 7.2). The average net magnetic flux density (signed) is estimated to be about $3.1 \mathrm{G}$ for $\mathrm{CH} 1$ and $1.0 \mathrm{G}$ for $\mathrm{CH} 2$. For a comparison, the magnetic fields measured by NSO/KP at the same day were also analyzed. The average net magnetic flux density is estimated to be about $4.4 \mathrm{G}$ for $\mathrm{CH} 1$ and $2.0 \mathrm{G}$ for $\mathrm{CH} 2$. The lower values determined by MDI data in both holes may result mainly from an underestimation of the net field strength. This is caused by the higher noise level of the field data measured by MDI. In that case part of the net flux contributed by weak magnetic fields may be cancelled if the field values are below the noise level. All the net flux densities of the photospheric magnetic are consistent with the previous work by Harvey et al. (1982). They found that average magnetic strengths ranged from $1 \mathrm{G}$ to $7 \mathrm{G}$ during the solar minimum.

A simple computation is performed to determine the parameter values of the plasma and magnetic field of the high-speed streams at $1 \mathrm{AU}$, by averaging the measured values over the least variable part of a high-speed stream between its leading and trailing edge. We believe that the central part of a high-speed stream can represent its average properties. The average proton flux and the radial component of the magnetic field are thus determined to be $2.6 \times 10^{8} \mathrm{~cm}^{-2} \mathrm{~s}^{-1}$ and $2.8 \mathrm{nT}$ for $\mathrm{CH} 1$, and $2.2 \times 10^{8} \mathrm{~cm}^{-2} \mathrm{~s}^{-1}$ and $2.7 \mathrm{nT}$ for $\mathrm{CH} 2$. These values are falling in the typical range of the parameters of a high-speed solar wind stream (see Table 2.1.).

Coronal parameters deduced from SUMER, MDI and NSO/KP and in situ parameters obtained by SWE and MFI are listed in Table 7.3 and in Table 7.4. By inspection of

Table 7.3: Coronal parameters deduced from SUMER, MDI and NSO/Kitt Peak

\begin{tabular}{|c|c|c|c|c|c|}
\hline & $\begin{array}{l}\mathrm{N}_{e} \\
\left(\mathrm{~cm}^{-3}\right)\end{array}$ & $\begin{array}{l}\mathrm{V} \\
\left(\mathrm{km} \mathrm{s}^{-1}\right)\end{array}$ & $\begin{array}{l}\mathrm{f}_{e}=\mathrm{N}_{e} * \mathrm{~V} \\
\left(\mathrm{~cm}^{-2} \mathrm{~s}^{-1}\right)\end{array}$ & $\begin{array}{l}\mathrm{B}_{0}(\mathrm{MDI}) \\
(\mathrm{G})\end{array}$ & $\begin{array}{l}\mathrm{B}_{0}(\mathrm{NSO} / \mathrm{KP}) \\
(\mathrm{G})\end{array}$ \\
\hline $\mathrm{CH} \overline{1}$ & $0.8 \times 10^{8}$ & 7.9 & $6.3 \times 10^{13}$ & 3.1 & 4.4 \\
\hline $\mathrm{CH} \overline{\overline{2}}$ & $1.8 \times 10^{8}$ & 1.4 & $2.5 \times 10^{13}$ & 1.0 & 2.0 \\
\hline
\end{tabular}


Table 7.4: In-situ parameters deduced from SWE and MFI

\begin{tabular}{lllll}
\hline & $\begin{array}{l}\mathrm{N}_{p} \\
\left(\mathrm{~cm}^{-3}\right)\end{array}$ & $\begin{array}{l}\mathrm{V}_{p} \\
\left(\mathrm{~km} \mathrm{~s}^{-1}\right)\end{array}$ & $\begin{array}{l}\mathrm{f}_{p}=\mathrm{N}_{p} * \mathrm{~V}_{p} \\
\left(\mathrm{~cm}^{-2} \mathrm{~s}^{-1}\right)\end{array}$ & $\begin{array}{l}\mathrm{B}_{r} \\
(\mathrm{nT})\end{array}$ \\
\hline $\mathrm{CH} 1$ & 4.3 & 606 & $2.6 \times 10^{8}$ & 2.8 \\
$\mathrm{CH} \overline{2}$ & 3.6 & 594 & $2.2 \times 10^{8}$ & 2.7 \\
\hline
\end{tabular}

Table 7.3 and Table 7.4, we can infer that the two high-speed streams produced by $\mathrm{CH} 1$ and $\mathrm{CH} 2$ have similar properties, particularly for the radial component of the magnetic field. If we trace back the proton flux and magnetic field under the assumption of a radial expansion of the streams, they should have an average proton flux of about $10^{13} \mathrm{~cm}^{-2} \mathrm{~s}^{-1}$ and a magnetic field of about $1.2 \mathrm{G}$ at the Sun's surface. Comparison with the photospheric magnetic field measured by MDI and NSO/KP indicates that $\mathrm{CH} 1$ has an expansion factor of about 3 derived from MDI data and 4 from NSO/KP data, and $\mathrm{CH} 2$ of about 1 inferred from MDI data and 2 from NSO/KP data. The larger expansion factor in $\mathrm{CH} 1$ may be interpreted as being due to $\mathrm{CH} 1$ having a stronger photospheric magnetic field. Again, as discussed above, the lower expansion factors determined by MDI data in both holes may mainly result from our underestimation of the net field strength caused by the noise level of the field data. On the other hand, if we determine the expansion factor by means of the mass flux measured by SUMER in the holes and by SWE at $1 \mathrm{AU}$, this expansion factor should be about 5 for $\mathrm{CH} 1$ and about 2 for $\mathrm{CH} 2$. It should be noted that the expansion factor referred to here is essentially the areal expansion between the coronal base and 1 $\mathrm{AU}$, so it can be considered as the global expansion factor.

In addition, measurements of the line widths show that the $\mathrm{Mg}$ IX and $\mathrm{Mg} \mathrm{X}$ lines have non-thermal velocities of $42 \mathrm{~km} \mathrm{~s}^{-1}$ and $22 \mathrm{~km} \mathrm{~s}^{-1}$ in $\mathrm{CH} 1$, and $33 \mathrm{~km} \mathrm{~s}^{-1}$ and $15 \mathrm{~km}$ $\mathrm{s}^{-1}$ in $\mathrm{CH} 2$, respectively. The widths of both coronal lines indicate that they have larger non-thermal velocities in $\mathrm{CH}$ 1 than in $\mathrm{CH} 2$.

\subsection{Summary and discussion}

In this chapter, measurements of the electron density in coronal holes, by using ultraviolet lines obtained with SUMER, have been discussed. We have also correlated coronal with in-situ measurements.

The electron densities in the transition region and at the coronal base have been determined by use of line pairs ( $\mathrm{Si}$ III, O V and Mg IX). The results were compared with previous measurements made by CDS and SUMER. Exploiting these results, the electron pressures can be estimated in the transition region and at the coronal base in the two holes analyzed. It is found that the electron pressures inferred by the two transition region lines are nearly constant. However, there exists a large thermal pressure gradient between the transition region and the coronal base. This pressure gradient may drive the initial outflow of the fast solar wind in coronal funnels. 
By direct comparison of the coronal and in situ observations, it is found that the global expansion factor of the solar wind stream tube is estimated to be about $4-5$ for $\mathrm{CH} 1$ and 2 for $\mathrm{CH} 2$. The factors are determined consistently from two independent groups of parameters, relating to the conservation of the mass flux and magnetic flux, respectively. It should be mentioned that the estimation of the plasma parameters (density and velocity) in coronal holes has a pronounced uncertainty, and the average net magnetic flux density determined by the photospheric magnetic field is roughly estimated, because of the difficulties in the determination of the hole boundaries. We also do not know how much of the magnetic flux within these holes is responsible for the interplanetary magnetic field observed at 1 AU. However, considering the conditions of the fast solar wind to be rather stable, the methods used here can still be considered reasonable.

The magnetic field strength and mass flux for the two holes are prominently different at the coronal base. They are both larger by a factor of about 2 in $\mathrm{CH} 1$ than in $\mathrm{CH} 2$, On the other hand, in situ observations shows that the velocity, mass flux and radial magnetic field strength of the streams produced by them are rather similar. The observational results of this study are in agreement with those obtained by a previous empirical study (Wang, 1995), which showed that the mass and energy flux densities in coronal holes tend to increase with the magnetic field strength at the coronal base and with the areal expansion factor. Theoretical models of the fast solar wind (Sandbæk et al., 1994) also indicated that the mass flux observed at $1 \mathrm{AU}$ could be nearly constant, if the mechanical energy flux heating the corona varied in proportion to the magnetic field strength at the coronal base. Moreover, the larger non-thermal velocities of the $\mathrm{Mg}$ IX and $\mathrm{Mg}$ X lines obtained in $\mathrm{CH} 1$ than in $\mathrm{CH} 2$ may also imply a larger mechanical energy deposition in $\mathrm{CH} 1$. 


\section{Chapter 8}

\section{Discussion: Implication to the Origin of the Fast Solar Wind}

\subsection{Fine structures and the network in coronal holes}

Like in the QS region, one of the most striking features observed by SUMER in ECHs is that small structures with an enhanced emission are present everywhere. The spatial distribution and the size of the fine structures have obviously a temperature-dependent property. Their appearances in the chromosphere and transition region are similar to those observed with SUMER in the QS region (Warren and Winebarger, 2000; Feldman et al., 2001).

In the chromosphere, such structures appear to be small bright patches with a size of about $2^{\prime \prime}-10^{\prime \prime}$, and are the predominant features in the network as well as in cell interiors. With the increase of the temperature, these features become more diffuse, and have an enlarged size. When the network seen in transition region lines, many of the bright structures appear to have a loop-like shape. They have visible widths of about $10^{\prime \prime}$ and lengths of about $10^{\prime \prime}-30^{\prime \prime}$, and can be seen more clearly in lines emitted by Li-like ions and also in the $\mathrm{H}$ I, He I and C III lines. In coronal holes, our observations show that many of such structures seem to have one footpoint rooted in the intranetwork and to extend into the cell interiors, and some of them appear as star-shape clusters. Moreover, their visible footpoints apparently correspond to the bright patches seen in chromospheric emissions. This result seems to be different from that observed in the quiet Sun by Feldman et al. (2001). They suggested that most of the loops straddle the chromospheric network and have no visible connections with bright patches seen in chromospheric lines. A possible interpretation is that such a difference may be due to the different geometry of the magnetic field in the two regions. Above the upper transition region, the most structures disappear and the corona becomes more homogeneous than in the QS region, except for some bright points. The results indicate that, at least in the chromosphere and the low transition region, the $\mathrm{CH}$ region shows more or less similar properties as in the QS region. A further implication is that the two regions may essentially be heated by a same mechanism, as suggested, e.g., by Marsch et al. (2003).

Comparing the network structure outlined by continuum emission with the magnetogram, the network seen in ultraviolet images spatially coincides with the locations, where the photospheric magnetic field flux is concentrated. As was expected, all coronal holes are dominated by a single polarity of the magnetic field, while there are also mixed-polarity features present. The magnetic flux of the opposite polarity occupies about $10 \%-40 \%$ of the total unsigned flux in the CHs. Larger mixed-polarity magnetic features, if can readily be distinguished in the magnetogram, usually correspond to locations with bright points 
seen in upper transition region and coronal lines. Moreover, the locations of these bright cores spatially coincide with those seen in the chromospheric lines, but with a brightness similar to the normal network. We have traced two examples of such bright points, and found that they were caused by the interaction of the closed magnetic loops with the unipolar fields in the coronal hole. On the other hand, many of bright structures had no connection with the intranetwork magnetic field having mixed polarities, as also found in the QS region by Warren and Winebarger (2000), which indicates that they are likely open into the corona.

According to previous model studies of the network (Dowdy et al., 1986; Dowdy, 1993), the network is believed to consist of both low-lying loops (less then $10^{4} \mathrm{~km}$ ) and largescale magnetic funnels being open into the corona. Very recently, a model sketch of the transition region structure has been suggested by Peter (2000, 2001), who studied several components of the transition region lines observed by SUMER in the quiet Sun, and found that a presence of the second broader spectral component is a general feature of transition region lines. This second component, with a broader and weaker profile, is suggested to originate from the large loops in the quiet Sun or open funnels in the coronal hole, while the core (first component) is from the smaller loops, and has a narrower line width and stronger intensity. Although we have not made a same analysis to deduce the second component, the positive correlation between the blue shift and the line width of the total profile has indeed been obtained. Thus, our results obtained in ECHs are generally in agreement with the prevailing network model, as suggested by various authors (see, e.g., Dowdy et al., 1986; Axford and McKenzie, 1992, 1997; Marsch and Tu, 1997; Peter, 2000, 2001).

Furthermore, our rapid-time-cadence (20 s) observations also show that the transition region and coronal base are not "quiet" but very dynamic, if seen in the Dopplergrams as well as the spectroheliograms. This result is consistent with the widely accepted conception that very-small-scale magnetic activities may be the ultimate source for heating the corona and accelerating the solar wind.

\subsection{Fine structures, spicules and plumes}

According to recent SUMER observations (Budnik et al., 1998; Wilhelm et al., 2000; Wilhelm, 2000), the visible structures observed above the limb are dominated by EUV spicules. They have a width of $10^{\prime \prime}-20^{\prime \prime}$ when seen in transition region lines, and disappear at a temperature above $0.5 \mathrm{MK}$. Geometrically, they appear to be nearly straight, but not as loops (Wilhelm, 2000). Spicules and macrospicules both have different orientations observed in polar $\mathrm{CH}$ regions (see the figures in Wilhelm, 2000; Wilhelm et al., 2000). The connection between the $\mathrm{H} \alpha$ and EUV spicules has not yet established. However, it is suggested that EUV spicules are very likely the hot sheath of cooler $\mathrm{H} \alpha$ spicules (Sterling, 2000). 
As was discussed above, many of the fine loop-like structures observed in ECHs have no connection with the intranetwork magnetic field having mixed polarities. As a possible explanation, Warren and Winebarger (2000) suggested that these fine features may be related to small loops connected to the internetwork (cell interiors) magnetic field or highly dynamic structures, such as spicules, which are not really loops. The possible solution of this difficulty may be to consider that both structures (loops and spicules) are present, both anchored in the network. On the one hand, the small loops are less extended in height (less then $10^{\prime \prime}$ ) and can not be distinguished if observed above the limb due to considerable overlapping of such loops (Warren and Winebarger, 2000). On the other hand, EUV spicules extend much higher (above $10^{\prime \prime}$ ), but can not readily be distinguished from the loops on the disk, due to either their projection effect or their overlapping with low-lying magnetic structures. Such a situation may really be true in coronal holes.

If we conjecture that the loop-like structures, which have a broader line width and blue shift seen in the O VI line in ECHs, are confined in a magnetic flux tube that is open to the corona, and that those with a narrower line width and a red shift are from the cooler magnetic loops that are closed locally, these results are in agreement with those obtained in the quiet Sun by Peter (2000, 2001). Moreover, if it is true that such structures with a bluer shift are open to the corona, it is possible that some of them will appear as visible EUV spicules or macrospicules if seen above the limb. On the disk, however, they may have projection with different lengths.

The bright points (BPs) seen in the transition region and coronal lines seem to have similar properties like those in polar coronal holes, where they have been identified as the base of the plumes. BPs have an enhanced emission, but have less blue shifts than the dark regions. Our estimate of the mass flux by measuring the Doppler shift is consistent with the previous estimate obtained for polar plumes by Wang (1994), and suggests that the portion of the mass flux contributed by bright points to the fast solar wind is at most comparable to the areal ratio of the BPs to the $\mathrm{CH}$ and thus negligible. Thus they may not be the main source of the fast solar wind due to their occupation of a smaller area.

\subsection{Doppler shifts and the nascent fast solar wind}

\section{Average blue shifts in the Ne VIII and Mg X lines}

The Doppler shifts derived from the Ne VIII and Mg X lines are on average blue in coronal holes. For the Ne VIII line, red shifts are present only in bright structures, with mixedpolarity magnetic structures underlying. Conversely, the larger blue shifts are mainly associated with the dark regions, where the strong magnetic flux of a single polarity is concentrated. On the other hand, the Dopplergram of the $\mathrm{Mg} \mathrm{X}$ line is more homogeneous in the hole region.

It seems that this is in agreement with a geometry of the hole consisting of coronal funnels, namely, the corona must be more uniform at a higher altitude. The results are in agreement 
with the notion that the fast solar wind is initially accelerated in such open magnetic funnels. The measured outflow velocity in the central region of the network is comparable with the values predicted by the models (see, e.g., Marsch and Tu, 1997; Hackenberg et al., 2000; Li, 2002; Vocks and Marsch, 2002). Moreover, an estimation of the mass flux, inferred by the blue shift of the $\mathrm{Mg} \mathrm{X}$ line and by the electron density measured in the Mg IX line, may also imply that the blue shifts measured in ECHs can represent the real outflow velocity of the nascent fast solar wind, although such a blue shift has also been interpreted alternatively as effects of nanoflare-induced waves propagating through coronal loops in the QS region (Hansteen, 1993; Wikstøl et al., 1997).

On the other hand, the Doppler shifts measured for the Ne VIII and $\mathrm{Mg} X$ lines in the quiet Sun are rather small, which usually fall in a range of $0 \pm 3 \mathrm{~km} \mathrm{~s}^{-1}$, as expected, and do not show prominent large-scale outflow there. They have also a narrower distribution when seen in the histogram of the line shifts. These results are reasonable, if considering that the most emission of the two lines is constrained to magnetic loops in the QS region, thereby without large-scale loops that may lead to a siphon flow.

\section{Doppler shifts of other lines}

Except for the Ne VIII and $\mathrm{Mg} X$ lines, all other transition region lines are on average red shifted in both $\mathrm{CH}$ and QS regions. Average red shifts of the transition region lines range mainly from $5 \mathrm{~km} \mathrm{~s}^{-1}$ to $10 \mathrm{~km} \mathrm{~s}^{-1}$ in the $\mathrm{CH}$ region, and $5 \mathrm{~km} \mathrm{~s}^{-1}$ to $15 \mathrm{~km} \mathrm{~s}^{-1}$ in the QS region. The temperature dependence of the average Doppler shifts, in both $\mathrm{CH}$ and QS regions, are consistent with previous results (Chae et al., 1998c; Peter and Judge, 1999; Teriaca et al. , 1999), i.e., the red shift increases first with increasing line formation temperature, but then drops again at a temperature of about $0.3 \mathrm{MK}$ (at the O VI line). Moreover, the average Doppler shifts measured are systematically bluer in the $\mathrm{CH}$ region than in the QS region. The difference of the Doppler shift between the two regions also shows a temperature-dependent property, which increases with increasing line formation temperature.

By inspection of the spatial distribution of the Doppler shifts, a clear positive correlation between the Doppler shifts for different lines can also be found. Moreover, the corresponding magnitudes of the Doppler shifts vary with different lines formed at different layers, for example, a larger value of the Doppler shift seen in the O VI line corresponds to a smaller one in the $\mathrm{C}$ II line and an even smaller one in the $\mathrm{H} \mathrm{I} \mathrm{L} \beta$ line.

In an individual structure, such tendency can be identified more clearly. For example, although the $\mathrm{O}$ VI line is on average red shifted in coronal holes, blue shifts can also be found in its Dopplergram, and tend to occur in the network where a large unipolar magnetic field is concentrated, which may be open to the corona. By inspection of the Dopplergrams of the $\mathrm{H} \mathrm{I} \mathrm{L} \beta$ and C II lines simultaneously recorded in the same spectral window, we can find that at the locations, where blue shifts are seen in the $\mathrm{O}$ VI line, in general, blue shifts are also seen in the $\mathrm{L} \beta$ line. For the $\mathrm{C}$ II line, the shifts often appear 
in Dopplergrams as small blue points with a smaller magnitude of shifts and a smaller spatial size.

The results may be important for us to understand the persistent red shifts and the process of the mass balance in the transition region. If a Doppler shift is considered to be caused by a steady mass flow, the results naturally agree with a convection pattern obey the law of mass-flux conservation in a flux tube. This means that the flow velocity should be lower in the chromosphere due to the higher plasma density there than in the transition region. On the other hand, a partial mass flux taken away by the solar wind would also reduce the average red shift of a line, even though the average Doppler shift is absolutely red (Pneuman and Kopp, 1978).

The persistent downflow would imply that plausible mechanisms should be given to interpret how this mass flux is injected into the transition region. One hypothesis has been proposed to explain this question by the return of spicular material (see,e.g., Pneuman and Kopp, 1978; Athay and Holzer, 1982; Athay, 1984; Cheng, 1992). Averaged over the Sun's surface, spicules carry an upward mass flux of about $4 \times 10^{-9} \mathrm{~g} \mathrm{~cm}^{-2} \mathrm{~s}^{-1}$ into the corona, if $1 \%$ areal coverage of spicules is considered. Compared with that, the mass flux taken away by the solar wind is about $3 \times 10^{-11} \mathrm{~g} \mathrm{~cm}^{-2} \mathrm{~s}^{-1}$. Because the portion taken away by the solar wind is only $1 \%$, most of the spicular material must fall back into the chromosphere. This idea is consistent with the fact that the globally averaged downward hydrogen flux inferred from UV observations is comparable to the upward hydrogen flux in spicules (Withbroe, 1983). However, it is not clear whether all the mass flux of the solar wind is contributed by such structures, even if this mechanism does work.

\subsection{Line width and heating mechanism}

For the line width in the $\mathrm{CH}$ region, the variation trend of lines formed below the upper transition region is more or less similar to that in the QS region. The difference between the two regions is small, with a slightly larger value in the hole. However, an apparent difference arises for the Ne VIII and Mg X lines. It should be noted that we just compare them in two regions observed within several days during solar maximum. In other coronal holes, the line width of the $\mathrm{Mg} \mathrm{X}$ line shows very large variation in different holes, and tends to increase with increasing strength of the underlying magnetic fields. Furthermore, the non-thermal velocity deduced from the Ne VIII and $\mathrm{Mg} \mathrm{X}$ lines in $\mathrm{CHs}$ at solar maximum are comparable with that measured above the limb in polar holes (Marsch et al. 1997; Doschek et al., 2001).

A line broadening can be caused by the combined effects of the differential bulk motion, thermal motion and wave/turbulence motion of the ions. Therefore, an exact interpretation of the resulting line width is difficult. However, evidence for preferential heating and acceleration of the ions very near the Sun have been found by observations with UVCS and SUMER (Kohl et al., 1997, 1998; Tu et al., 1998). This supports the idea that dissipation of high-frequency Alfvén waves in coronal funnels may be a prime candidate for 
heating of the magnetically open corona (see, e.g., Marsch, 1992; Axford and McKenzie, 1992, 1997; Marsch and Tu, 1997). If the increase of the line width can be interpreted as being caused by enhanced wave/turbulence motions, our results seem to indicate that the wave-mechanical energy flux correlates with the strength of the magnetic field in coronal holes.

\subsection{Magnetic field and the global fast solar wind}

The quantity of $v \sqrt{I}$ (with $I \sim n_{e}^{2}$ ) has been used as a measure of the coronal mass flux of the nascent solar wind in our study. A clear positive correlation between the mass flux and the magnetic field flux density has been found by analyzing five ECHs. If this estimation of the mass flux ratio between different holes is reliable, it would place an important observational constraint on the mass flux. Our findings indicate that the mass flux of the nascent fast solar wind may be directly associated with the net flux density of the magnetic field. This can also be supported by direct estimation of the mass flux inferred by the blue shift of the $\mathrm{Mg} X$ line and by the electron density measured in the $\mathrm{Mg}$ IX line.

Because the properties of the fast solar wind has been demonstrated to be rather similar in Earth's orbit, as we have also found in our case studies, a coronal hole with a larger mass flux at the coronal base must have a larger global expansion of the solar wind stream. This can be supported by our estimation of the expansion factor inferred by the magnetic fields observed at the coronal base and in Earth's orbit, relating to the conservation of the magnetic flux. Our results are also in agreement with those obtained by a previous empirical study (Wang, 1995), which showed that the mass and energy flux densities in coronal holes tend to increase with the magnetic field strength at the coronal base and with the areal expansion factor. Theoretical models of the fast solar wind (Sandbæk et al., 1994) also indicated that the mass flux observed at 1 AU could be nearly constant, if the mechanical energy flux heating the corona varied in proportion to the magnetic field strength at the coronal base. This may also be implied by the positive correlation between the line width of the $\mathrm{Mg} X$ line and the strength of the underlying magnetic field in the $\mathrm{ECH}$, as we have discussed above. 


\section{Chapter 9}

\section{Summary}

In this thesis, the general properties of equatorial coronal holes and their relation to the high-speed solar wind streams have been investigated, by combining the remote sensing observations with SUMER, EIT, MDI and NSO/KP as well as in situ observations of the solar wind with SWE and MFI onboard WIND. We summarize important results and conclude as follows:

\section{Morphological results}

- Equatorial CHs seen in chromospheric lines have generally similar properties as the normal QS region. Small bright patches with a size of about $2^{\prime \prime}-10^{\prime \prime}$ are the predominant features in the network as well as in cell interiors. With the increase of the temperature, these features become less pronounced.

- The network width as seen in all transition region lines appears to be broader in CHs than in the QS region. Loop-like structures are the most prominent features in the transition region, in both $\mathrm{CH}$ and QS regions. They have visible widths of about $10^{\prime \prime}$ and lengths of about $10^{\prime \prime}-30^{\prime \prime}$, and can be seen more clearly in lines emitted by Li-like ions and also in the $\mathrm{H} \mathrm{I}, \mathrm{He} \mathrm{I}$ and $\mathrm{C}$ III lines. In $\mathrm{CHs}$, we found that many of such structures seem to have one footpoint rooted in the intranetwork and to extend into the cell interiors. Some of them appear as star-shape clusters.

- In Dopplergrams of the O VI line, fine structures with apparent blue shifts are also present, although on average they are red shifted. Some of the structures appear as looplike and spatially coincide with the locations of the loop-like structures seen in intensity images, while some appear as blue points, which have less extension and are located at the same footpoints of the loop-like structures seen in intensity images. The blue structures usually have a broader line width. They seem to represent plasma above the concentration of a large unipolar magnetic field, without obvious bipolar magnetic features nearby. Moreover, the locations with blue shifts seen in the $\mathrm{O}$ VI line are usually also blue shifted if seen in the $\mathrm{L} \beta$ line, and for the $\mathrm{C}$ II line, they often appear as blue points with a smaller magnitude of shifts and a smaller spatial size.

- Some CHs have a very different appearance as seen in the Ne VIII line in comparison with as observed in the Fe XII and Fe XV EIT channels. We suggest that this difference may have two reason: First, the average magnetic flux density (signed) is small in such CHs. Second, there are many small bipolar magnetic structures present there, leading to formation of small bright structures that dominate the emission observed in lines with lower formation temperatures, but such bright structures eventually disappear with the increase of temperature, and thus are not observed in the coronal channels of EIT. 
- The Doppler shifts derived from the Ne VIII and Mg X lines are on average blue in CHs. Red shifts are present only in bright structures, with mixed-polarity magnetic structures underlying. Conversely, the larger blue shifts are mainly associated with the dark regions, where the strong magnetic flux of a single polarity is concentrated. On the other hand, the Dopplergram of the $\mathrm{Mg} \mathrm{X}$ line is more homogeneous in the hole area. It seems that this is in agreement with a geometry of the hole consisting of coronal funnels, namely, the corona must be more uniform at a higher altitude. The results are in agreement with the notion that the fast solar wind is initially accelerated in such open magnetic funnels. The measured outflow velocity in the central region of the network is comparable with the values predicted by the models.

\section{Statistical and quantitative results}

- From the histogram plots of the line parameters, the intensity distribution shows that a slight difference between $\mathrm{CH}$ and QS regions has already presented from the upper chromosphere. A prominent difference can be seen in the transition region lines, in particular for the Li-like ions and helium. The apparent higher counts in the high-intensity tail for the Li-like ions and helium are consistent with the morphological results that the loop-like structures of these lines are more extended into the cell interiors in CHs than in the QS region.

- The average intensity is lower for transition region and coronal lines in $\mathrm{CH}$ s than in the QS region. The intensity ratio between $\mathrm{CH}$ and QS regions generally decreases with the formation temperature, in agreement with previous studies. Such a decrease of the intensity ratio is found to be more clearly for the helium and Li-like ions.

- The distribution of the Doppler shift exhibits a more spread profile for the O VI, Ne VIII and $\mathrm{Mg} X$ lines with a high formation temperature, while for other cooler lines it shows a same trend, but less pronounced.

- Except for the Ne VIII and Mg X lines, all other lines are on average red shifted in both $\mathrm{CH}$ and QS regions. Average red shifts of the transition region lines range mainly from $5 \mathrm{~km} \mathrm{~s}^{-1}$ to $10 \mathrm{~km} \mathrm{~s}^{-1}$ in the $\mathrm{CH}$ region, and $5 \mathrm{~km} \mathrm{~s}^{-1}$ to $15 \mathrm{~km} \mathrm{~s}^{-1}$ in the QS region. Small Doppler shifts are measured for the Ne VIII and Mg X lines in the QS region. The temperature-dependence of average Doppler shifts in both $\mathrm{CH}$ and QS regions are consistent with previous results. Moreover, transition region lines have systematically smaller red shifts in $\mathrm{CHs}$ than in the QS region. The difference of the Doppler shift between $\mathrm{CH}$ and QS regions increases with increasing line formation temperature.

- The distribution of the line width reveals a slightly wider value (with a larger value of around 1-2 $\mathrm{km} \mathrm{s}^{-1}$ ) for all lines formed at a temperature below $0.5 \mathrm{MK}$ in CHs. The trend of non-thermal velocity varying with the temperature is similar to the one in the QS region, as found by other authors. Obviously larger line widths have been measured for the $\mathrm{Ne}$ VIII and $\mathrm{Mg}$ X lines in $\mathrm{CH} 6$ than in QS1, which were observed at a same solar active level. However, the line width of the $\mathrm{Mg} \mathrm{X}$ line shows very large variation in different holes. 
- It is found that an apparent correlation between the line parameters and the chromospheric network is present for the most measured lines. The chromospheric line of Si II reveals a very clear positive correlation between its Doppler shift, intensity and line width. For the He I line and transition region lines, such a relation becomes more complicated. In general, a larger blue or red shift usually has a larger line width and intensity, and also corresponds to a larger intensity of continuum, which implies that they mainly occur in the network. In the Ne VIII line, there exists a slightly positive correlation between the Doppler shift and intensity. A bluer shift tends to have a lower intensity and a redder one corresponds to a larger intensity. For the line width, the tendency reveals to be inversely. The results are in agreement with those obtained in polar CHs.

- There exists a clear positive correlation between Doppler shifts deduced from different lines which were observed simultaneously in the same spectral window. Moreover, the corresponding magnitudes of the Doppler shift vary with different lines formed at different layers, i.e., a larger value of the Doppler shift seen in the O VI line corresponds to a smaller one in the C II line and an even smaller one in the $\mathrm{H} \mathrm{I} \mathrm{L} \beta$ line. This statistical result nicely confirms the result obtained in the morphological study.

- The line width deduced from the $\mathrm{Mg} \mathrm{X}$ line in various $\mathrm{CHs}$ shows a clear trend to increase with the increasing underlying magnetic field strength (signed). Moreover, the quantity of $v \sqrt{I}$ (with $I \sim n_{e}^{2}$ ), which is used as a proxy for the coronal mass flux of the nascent fast solar wind, also reveals a clear positive correlation to the magnetic field strength.

\section{Comparison of coronal and in situ observations}

- The electron densities in the transition region and at the coronal base have been determined by use of line pairs ( $\mathrm{Si}$ III, O V and Mg IX). The results were compared with previous measurements made by CDS and SUMER. Exploiting these results, the electron pressures can be estimated in the transition region and at the coronal base in the two holes analyzed. It is found that the electron pressures inferred by the two transition region lines are nearly constant. However, there exists a large thermal pressure gradient between the transition region and the coronal base. This pressure gradient may drive the initial outflow of the fast solar wind in coronal funnels.

- By direct comparison of the coronal and in situ observations, it is found that the global expansion factor of the solar wind stream tube is estimated to be about 4-5 for $\mathrm{CH} 1$ and 2 for $\mathrm{CH} 2$. The factors are determined consistently from two independent groups of parameters, relating to the conservation of the mass flux and magnetic flux, respectively. 



\section{Bibliography}

Ahmad, I. A. and D. F. Webb. "X-ray analysis of a polar plume". Solar Phys., 58: 323336, 1978.

Ahmad, I. A. and G. L. Withbroe. "EUV analysis of polar plumes". Solar Phys., 53: 397-408, 1977.

Andretta, V. and H. P. Jones. "On the role of the solar corona and transition region in the excitation of the spectrum of neutral helium". Astrophys. J., 489: 375-394, 1997.

Aschwanden, M. J. and L. W. Acton. "Temperature Tomography of the Soft X-Ray Corona: Measurements of Electron Densities, Temperatures, and Differential Emission Measure Distributions above the Limb”. Astrophys. J., 550: 475-492, 2001.

Athay, R. G. "The origin of spicules and heating of the lower transition region". Astrophys. J., 287: 412-417, 1984.

Athay, R. G., J. B. Gurman and W. Henze. "Fluid motions in the solar chromospherecorona transition region. III - Active region flows from wide slit Dopplergrams". Astrophys. J., 269: 706-714, 1983a.

Athay, R. G., J. B. Gurman, W. Henze and R. A. Shine. "Fluid motions in the solar chromosphere-corona transition region. I - Line widths and Doppler shifts for C IV". Astrophys. J., 265: 519-529, 1983b.

Athay, R. G. and T. E. Holzer. "The role of spicules in heating the solar atmosphere". Astrophys. J., 255: 743-752, 1982.

Axford, W. I. and J. F. McKenzie. "The origin of the high speed solar wind streams". In: E. Marsch and R. Schwenn, eds., "Solar Wind Seven", pages 1-5. Pergamon Press, Oxford, England, 1992.

Axford, W. I. and J. F. McKenzie. "The Solar Wind". In: J. R. Jokipii, C. P. Sonett and M. S. Giampapa, eds., "Cosmic Winds and the Heliosphere", pages 31-66. The University of Arizona Press, Tucson, 1997.

Axford, W. I., J. F. McKenzie, G. V. Sukhorukova, M. Banaszkiewicz, A. Czechowski et al. "Acceleration of the high speed solar wind in coronal holes". Space Sci. Rev., 87: 25-41, 1999.

Banaszkiewucz, M., W. I. Axford and J. F. McKenzie. "An analytic solar magnetic field model". Astron. \& Astrophys., 337: 940-944, 1998. 
Barrow, J. D., S. P. Bhavsar and D. H. Sonoda. "A bootstrap resampling analysis of galaxy clustering”. Mon. Not. R. Astro. Soc., 210: 19-23, 1984.

Barsi, G. S., J. L. Linskey, J.-D. F. Bartoe, G. E. Brueckner and M. E. Van Hoosier. "Lyman-alpha rocket spectra and models of the quiet and active solar chromosphere based on partial redistribution diagnostics". Astrophys. J., 230: 924-949, 1979.

Bartoe, J.-D. F. and G. E. Brueckner. "New stigmatic, coma-free, concave-grating spectrograph". J. Opt. Soc. America, 65: 13-21, 1975.

Baum, W. A., F. S. Johnson, J. J. Oberly, C. C. Rockwood, C. V. Strain et al.. "Solar ultraviolet spectrum to 88 kilometers". Phys. Rev., 70: 781-782, 1946.

Beckers, J. M. "Solar spicules”. Solar Phys., 3: 367-433, 1968.

Beckers, J. M. “Solar spicules”. Ann. Rev. Astron. Astrophys., 10: 73-100, 1972.

Belenko, I. A. "Coronal hole evolution during 1996-1999”. Solar Phys., 199: 23-35, 2001.

Bocchialini, K. and J. C. Vial. "High-chromosphere and low-transition-region network: a different organization in an equatorial coronal hole?" Solar Phys., 168: 37-45, 1996.

Bohlin, J. D. "Extreme-Ultraviolet Observations of Coronal Holes I: Locations, Sizes and Evolution of Coronal Holes, June 1973-January 1974”. Solar Phys., 51: 377-398, $1977 \mathrm{a}$.

Bohlin, J. D. "An observation definition of coronal holes". In: J.B. Zirker, ed., "Coronal holes and high speed wind streams", pages 27-70. Colorado Associated University Press, Boulder, 1977b.

Bohlin, J. D. and N. R. Jr. Sheeley. "Extreme-ultraviolet observations of coronal holes II: Association of holes with solar magnetic fields and a model for their formation during the solar cycle". Solar Phys., 56: 125-151, 1978.

Bohlin, J. D., N. R. Jr. Sheeley and R. Tousey. "Structure of the Sun's polar cap at wavelengths 240-600 ̊̊”. In: M. J. Rycroft, ed., "Space research", pages 651-656. Akademie Verlag, Berlin, 1975.

Boris, J. P. and J. T. Mariska. "An explanation for the systematic flow of plasma in the solar transition region”. Astrophys. J., 258: L49-L52, 1982.

Bravo, S. and G. A. Stewart. "The Correlation Between Sunspot and Coronal Hole Cycles and a Forecast of the Maximum of Sunspot Cycle 23". Solar Phys., 173: 193-198, 1997.

Brekke, P. “Observations of Transition Region Plasma”. Solar Phys., 190: 379-408, 1999. 
Brekke, P., D. M. Hassler and K. Wilhelm. "Doppler shifts in the quiet-Sun transition region and corona observed with SUMER on SOHO”. Solar Phys., 175: 349-374, 1997.

Bromage, B. J. I., D. Alexander, A. Breen, J. R. Clegg, G. Del Zanna et al.. "Structure of a large low-latitude coronal hole”. Solar Phys., 193: 181-193, 2000.

Broussard, R. M., N. R. Jr. Sheeley, P. Tousey and J. H. Underwood. "A survey of coronal holes and their solar wind associations throughout sunspot cycle 20". Solar Phys., 56: 161-183, 1978.

Brueckner, G. E. and J.-D. F. Bartoe. "Observations of high-energy jets in the corona above the quiet Sun, the heating of the corona, and the acceleration of the solar wind". Astrophys. J., 272: 329-348, 1983.

Brueckner, G. E., J.-D. F. Bartoe, J. W. Cook, K. P. Dere and D. G. Socker. "HRTS results from Spacelab 2". Adv. Space Res., 6: 263-272, 1986.

Brynildsen, N., P. Brekke, T. Fredvik, S. V. H. Haugan, O. Kjeldseth-Moe et al.. "SOHO observations of the connection between line profile parameters in active and quiet regions and the net red shift in EUV emission lines". Solar Phys., 181: 23-50, 1998.

Budnik, F., K.-P. Schröder, K. wilhelm and K.-H. Glassmeier. "Observational evidence for coronal mass injection by "evaporation" of spicular plasma". Astron. \& Astrophys., 334: L77-L80, 1998.

Carlsson, M., P. G. Judge and K. Wilhelm. "SUMER observations confirm the dynamic nature of the quiet solar outer atmosphere: The internetwork chromosphere". Astrophys. J., 486: L63-L66, 1997.

Chae, J., U. Schühle and P. Lemaire. "SUMER Measurements of Nonthermal Motions: Constraints on Coronal Heating Mechanisms". Astrophys. J., 505: 957-973, 1998 a.

Chae, J., H. Wang, C.-Y. Lee, P. R. Goode and U. Schühle. "Photospheric magnetic field changes associated with transition region explosive events". Astrophys. J., 497: L109L112, 1998b.

Chae, J., H. S. Yun and A. I. Poland. "Temperature dependence of ultraviolet line average doppler shifts in the quiet Sun”. Astrophys. J. Suppl., 114: 151-164, 1998c.

Charbonneau, P. "Genetic algorithms in astronomy and astrophysics". Astrophys. $J$. Suppl., 101: 309-334, 1995.

Cheng, Q. Q. "Fluid motions in the solar atmosphere. III - A possible explanation of the downflows". Astron. \& Astrophys., 262: 581-586, 1992.

Cranmer, S. R. "Coronal holes and the high-speed solar wind". Space Sci. Rev., 101: 229-294, 2002. 
Cranmer, S. R., J. L. Kohl, G. Noci, E. Antonucci, G. Tondello et al.. "An empirical model of a polar coronal hole at solar minimum". Astrophys. J., 511: 481-501, 1999.

Curdt, W., P. Brekke, U. Feldman, K. Wilhelm, B. N. Dwivedi et al. "The SUMER spectral atlas of solar-disk features". Astron. \& Astrophys., 375: 591-613, 2001.

Curdt, W., U. Feldman, J. M. Laming, K. Wilhelm, U. Schühle et al. . "The solar disk spectrum between 660 and 1175 Anstroms (first order) obtained by SUMER on SOHO". Astron. \& Astrophys. Suppl. Ser., 126: 281-296, 1997.

Curdt, W. and P. Heinzel. "Dynamic behavior of the upper solar atmosphere: SUMER/SOHO observations of hydrogen Lyman lines". Astrophys. J., 503: L95-L100, 1998.

Cushman, G. W. and W. A. Rense. "Evidence of outward flow of plasma in a coronal hole”. Astrophys. J., 207: L61-L62, 1976.

Cushman, G. W. and W. A. Rense. "Erratum: Evidence of outward flow of plasma in a coronal hole". Astrophys. J., 211: L57, 1977.

Dammasch, I. E., D. M. Hassler, W. Curdt and K. Wilhelm. "Statistical analysis of EUV and UV lines inside and outside of solar coronal holes". Space Sci. Rev., 87: 161-164, 1999a.

Dammasch, I. E., D. M. Hassler, K. Wilhelm and W. Curdt. "Solar Mg X and Fe XII wavelengths measured by SUMER". Proc. 8th SOHO Workshop, ESA SP-446: 263268, 1999b.

Dammasch, I. E., K. Wilhelm, W. Curdt and D. M. Hassler. "The Ne VIII $(\lambda 770)$ resonance line: solar wavelengths determined by SUMER on SOHO". Astron. \& Astrophys., 346: 285-294, 1999c.

David, C., A. H. Gabriel, F. Bely-Dubau, A. Fludra, P. Lemaire et al.. "Measurements of the electron temperature gradient in a solar coronal hole". Astron. \& Astrophys., 346: L90-L94, 1998.

Deforest, C. E., J. T. Hoeksema, J. B. Gurman, B. J. Thompson, S. P. Plunkett et al. . "Polar plume anatomy: Results of a coordinated observation". Space Sci. Rev., 175: 393-410, 1997.

Deforest, C. E., S. P. Plunkett and M. D. Andrews. "Observation of polar plumes at high solar altitudes". Astrophys. J., 546: 569-575, 2001.

Del Zanna, G. and B. J. I. Bromage. "The elephant's trunk: Spectroscopic diagnostics applied to SOHO/CDS observations of the August 1996 equatorial coronal hole". $J$. Geophys. Res., 104: 9753-9766, 1999. 
Delaboudinière, J.-P., G. E. Artzner, J. Brunaud, A. H. Gabriel, J. F. Hochedez et al.. "EIT: extreme-ultraviolet imaging telescope for the SOHO mission". Solar Phys., 162: 291-312, 1995.

Dere, K. P., J.-D. F. Bartoe and G. E. Brueckner. "High-resolution telescope and spectrograph observations of the quiet solar chromosphere and transition zone". Astrophys. J., 281: 870-883, 1984.

Dere, K. P., J.-D. F. Bartoe and G. E. Brueckner. "Explosive events in the transition zone". Solar Phys., 123: 41-68, 1989a.

Dere, K. P., J.-D. F. Bartoe and G. E. Brueckner. "Explosive events and magnetic reconnection in the solar atmosphere". J. Geophys. Res., 96: 9399-9407, 1991.

Dere, K. P., J.-D. F. Bartoe, G. E. Brueckner, J. W. Cook and D. G. Socker. "Ultraviolet observations of solar fine structure". Science, 238: 1267-1269, 1987.

Dere, K. P., J.-D. F. Bartoe, G. E. Brueckner and F. Recely. "Transition zone flows observed in a coronal hole on the solar disk". Astrophys. J., 345: L95-L97, 1989b.

Dere, K. P., E. Landi, H. E. Mason, B. C. Monsignori Fossi and P. R. Young. "CHIANTI an atomic database for emission lines". Astron. \& Astrophys. Suppl. Ser., 125: 149-173, 1997.

Domingo, V., B. Fleckb and A. I. Poland. "The SOHO mission: An overview". Solar Phys., 162: 1-37, 1995.

Dorotovic, I. "Area of polar coronal holes and sunspot activity: years 1939-1993". Solar Phys., 167: 419-426, 1996.

Doschek, G. A. "The effects of a multidensity plasma on ultraviolet spectroscopie electron density diagnostics". Astrophys. J., 279: 446-452, 1984.

Doschek, G. A., U. Feldman and J. D. Bohlin. "Doppler wavelength shifts of transition zone lines measured in SKYLAB solar spectra". Astrophys. J., 205: L177-L180, 1976.

Doschek, G. A., U. Feldman, J. Laming, U. Schühle and K. Wilhelm. "Properties of solar polar coronal hole plasmas observed above the limb". Astrophys. J., 546: 559-568, 2001.

Doschek, G. A., U. Feldman, J. M. Laming, H. P. Warren, U. Schühle et al.. "The electron pressure in the solar lower transition region determined from O V and Si III densitysensitive line ratios". Astrophys. J., 507: 991-996, 1998.

Doschek, G. A., U. Feldman and R. Tousey. "Limb-brightening curves of XUV transition zone lines in the quiet Sun and in a polar coronal hole observed by Skylab". Astrophys. J., 202: L151-L154, 1975. 
Dowdy, J. F. Jr. "Observational evidence for hotter transition region loops within the supergranular network". Astrophys. J., 411: 406-409, 1993.

Dowdy, J. F. Jr., D. Rabin and R. L. Moore. "On the magnetic structure of the quiet transition region”. Solar Phys., 105: 35-45, 1986.

Doyle, J. G., F. P. Keenan, R. S. I. Ryans, K. M. Aggarwal and A. Fludra. "Electron densities above a polar coronal hole based on improved Si IX density diagnostics". Solar Phys., 188: 73-80, 1999.

Drago, F. Chiuderi, E. Landi, A. Fludra and A. Kerdraon. "EUV and radio observations of an equatorial coronal hole". Astron. \& Astrophys., 348: 261-270, 1999.

Dulk, G. A. and K. V. Sheridan. "The structure of the middle corona from observations at 80 and $160 \mathrm{Mhz".} \mathrm{Solar} \mathrm{Phys.,} \mathrm{36:} \mathrm{191-202,} 1974$.

Dupree, A. K., M. J. Penn and H. P. Jones. "He I 10830 Åwing asymmetry in polar coronal holes: evidence for radial outflows”. Astrophys. J., 467: L121-L124, 1996.

Edlén, B. Nova Acta Reg. Soc. Sci. Uppsala (IV), 9: 6, 1934.

Esser, R. and R. J. Edgar. "Reconciling spectroscopic electron temperature measurements in the solar corona with in situ charge state observations". Astrophys. J., 532: L71-L74, 2000 .

Esser, R. and S. R. Habbal. "Coronal holes and the solar wind". In: J. R. Jokipii, C. P. Sonett and M. S. Giampapa, eds., "Cosmic Winds and the Heliosphere", pages 297326. The University of Arizona Press, Tucson, 1997.

Feldman, U., W. Curdt, E. Landi and K. Wilhelm. "Identification of spectral lines in the 500-1600 A wavelength range of highly ionized $\mathrm{Ne}, \mathrm{Na}, \mathrm{Mg}, \mathrm{Ar}, \mathrm{K}, \mathrm{Ca}, \mathrm{Ti}, \mathrm{Cr}, \mathrm{Mn}$, $\mathrm{Fe}, \mathrm{Co}$, and $\mathrm{Ni}$ emitted by flares $\left(T_{e} \geq 3 \times 10^{6} \mathrm{~K}\right)$ and their potential use in plasma diagnostics". Astrophys. J., 544: 508-521, 2000a.

Feldman, U., I. E. Dammasch and K. Wilhelm. "The Morphology of the solar upper atmosphere during the sunspot minimum". Space Sci. Rev., 93: 411-472, 2000b.

Feldman, U., I. E. Dammasch and K. Wilhelm. "On the unresolved fine structures of the solar upper atmosphere. IV. the interface with the chromosphere". Astrophys. J., 558: 423-427, 2001.

Feldman, U., G. A. Doschek and L. Cohen. "Doppler wavelength shifts of ultraviolet spectral lines in solar active regions". Astrophys. J., 255: 325-328, 1982.

Fontenla, J. M., E. H. Avrett and R. Loeser. "Energy balance in the solar transition region. IV. Hydrogen and helium mass flows with diffusion". Astrophys. J., 572: 636-662, 2002. 
Forsyth, R. J., A. Balogh, T. S. Horburg, G. Erdös, E. J. Smith et al.. "The heliospheric magnetic field at solar minimum: Ulysses observations from pole to pole". Astron. \& Astrophys., 316: 287-295, 1996.

Gabriel, A. H. "A magnetic model of the solar transition region". Phil. Trans. Roy. Soc., A281: 339-352, 1976.

Gallagher, P. T., K.J.H. Philips, L.K. Harra-Murnion and F.P.Keenan. "Properties of the quiet Sun EUV network”. Astron. \& Astrophys., 335: 733-745, 1998.

Gebbie, K. B., F. Hill, L. J. November, J. B. Gurman, R. A. Shine et al.. "Steady flows in the solar transition region observed with SMM". Astrophys. J., 251: L115-L118, 1981.

Geiss, J., G. Gloeckler, R. von Steiger, H. Balsiger, L. A. Fisk et al.. "The southern high-speed stream - Results from the SWICS instrument on ULYSSES”. Science, 268: 1033-1036, 1995.

Gibson, S. E. "Global solar wind structure from solar minimum to solar maximum: sources and evolution”. Space Sci. Rev., 97: 69-79, 2001.

Golub, L., A. S. Krieger, J. W. Harvey and G. S. Vaiana. "Magnetic properties of X-ray bright points". Solar Phys., 53: 111-121, 1977.

Golub, L., A. S. Krieger and G. S. Vaiana. "Distribution of lifetimes for coronal soft X-ray bright points". Solar Phys., 49: 79-90, 1976.

Gopalswamy, N., K. Shibasaki, B. J. Thompson, J. B. Gurman and C. E. DeForest. "Is the chromosphere hotter in coronal holes". In: S. R. Habbal and et al., eds., "Solar Wind Nine", pages 277-280. The American Institute of Physics, USA, 1999.

Gouttebroze, P., P. Lemaire, J. C. Vial and G. Artzner. "The solar hydrogen Lymanbeta and Lyman-alpha lines - Disk center observations from OSO 8 compared with theoretical profiles". Astrophys. J., 225: 655-664, 1978.

Griesmann, U. and R. Kling. "Interferometric measurement of resonance transition wavelengths in C IV, Si IV, Al III, Al II, and Si II”. Astrophys. J., 536: L113-L115, 2000.

Griffiths, N. W., G. H. Fisher, D. T. Woods and O. H. W. Siegmund. "SUMER Observations of the Quiet-Sun Transition Region”. Astrophys. J., 512: 992-1005, 1999.

Gringauz, K. I., V. V. Bezrukikh, V. D. Ozerov and R. E. Rybchinskii. "A study of the interplanetary gas, high-energy electrons, and corpuscular radiation from the Sun by means of a three-electrode trap for charged particles on the Soviet cosmic rocket". Sov. Phys. Dokl. (English translation), 5: 361, 1960.

Grossmann-Doerth, U. and W. Schmidt. "Chromospheric fine structure revisited". Astron. \& Astrophys., 264: 236-242, 1992. 
Habbal, S. R. "Coronal energy distribution and X-ray activity in the small scale magnetic field of the quiet Sun”. Ann. Geophysicae, 10: 34-46, 1992.

Habbal, S. R., J. F. Jr. Dowdy and G. Withbroe. "A Comparison between bright points in a coronal hole and a quiet-Sun region”. Astrophys. J., 352: 333-342, 1990.

Habbal, S. R., R. Esser and M. B. Arndt. "How reliable are coronal hole temperatures deduced from observations?" Astrophys. J., 413: 435-444, 1993.

Hackenberg, P., E. Marsch and G. Mann. "On the origin of the fast solar wind in polar coronal funnels”. Astron. \& Astrophys., 360: 1139-1147, 2000.

Hammer, R. "Energy balance of stellar coronae. I - Methods and examples. II - Effect of coronal heating". Astrophys. J., 259: 767-791, 1982.

Hansteen, V. H. "A new interpretation of the redshift observed in optically thin transition region lines”. Astrophys. J., 402: 741-755, 1993.

Hansteen, V. H., R. Betta and M. Carlsson. "Rapid intensity and velocity variations in solar transition region lines". Astron. \& Astrophys., 360: 742-760, 2000.

Hansteen, V. H. and E. Leer. "Coronal heating, densities, and temperatures and solar wind acceleration”. J. Geophys. Res., 100: 21577-21593, 1995.

Harvey, J. W. "Coronal Polar Rays and Polar Magnetic Fields". Astrophys. J., 141: 832834, 1965.

Harvey, J. W., A. S. Krieger, A. F. Timothy and G. S. Vaiana. "Comparison of Skylab X-ray and ground-based helium observations”. Bull. American Astron. Soc., 7: 358, 1975.

Harvey, K. L., N. R. Jr. Sheeley and J. W. Harvey. "Magnetic measurements of coronal holes during 1975-1980”. Solar Phys., 79: 149-160, 1982.

Hassler, D. M ., G. J. Rottman and F. Q. Orrall. "Systematic radial flows in the chromosphere, transition region, and corona of the quiet sun". Astrophys. J., 372: 710-718, 1991.

Hassler, D. M., I. E. Dammasch, P. Lemaire, P. Brekke, W. Curdt et al.. "Solar wind outflow and the chromospheric magnetic network". Science, 283: 810-813, 1999.

Hassler, D. M., G. J. Rottman, E. C. Shoub and T. E. Holzer. "Line broadening of Mg X 609 and 625 A coronal emission lines observed above the solar limb". Astrophys. J., 348: L77-L80, 1990.

Hassler, D. M., K. Wilhelm, P. Lemaire and U. Schühle. "Observations of polar plumes with the SUMER instrument on SOHO”. Solar Phys., 175: 375-391, 1997. 
Holzer, T. E. and W. I. Axford. "The theory of stellar winds and related flows". Ann. Rev. Astron. Astrophys., 8: 31-60, 1970.

Howard, R. and B. J. LaBonte. "Surface magnetic fields during the solar activity cycle". Solar Phys., 74: 131-145, 1981.

Huber, M. C. E., A. K. Dupree, L. Goldberg, R. W. Noyes, W. H. Parkinson et al.. "The Harvard Experiment on OSO-6: Instrumentation, calibration, operation, and description of observations". Astrophys. J., 183: 291-312, 1973.

Huber, M. C. E., P. V. Foukal, R. W. Noyes, E. M. Reeves, E. J. Schmahl et al. ."Extremeultraviolet observations of coronal holes: Initial results from SKYLAB". Astrophys. J., 194: L115-L118, 1974.

Hundhausen, A. J. "An interplanetary view of coronal holes". In: J.B. Zirker, ed., "Coronal holes and high speed wind streams", pages 221-329. Colorado Associated University Press, Boulder, 1977.

Inhester, B., G. Stenborg, R. Schwenn, N. Srivastava and B. Podlipnik. "The rotation of the Fe XIV solar corona during the recent solar activity minimum”. Space Sci. Rev., 87: 211-214, 1999.

Innes, D. E., P. Brekke, D. Germerott and K. Welhlem. "Bursts of explosive events in the solar network". Solar Phys., 175: 341-348, 1997a.

Innes, D. E., B. Inhester, W. I. Axford and K. Welhlem. "Bi-directional plasma jets produced by magnetic reconnection on the Sun". Nature, 386: 811-813, $1997 \mathrm{~b}$.

Insley, J.E., V. Moore and R.A. Harrison. "The differential rotation of the corona as indicated by coronal holes". Solar Phys., 160: 1-18, 1995.

Jones, H. P., T. L. Jr. Duvall, J. W. Harvey, C. T. Mahaffey, J. D. Schwitters et al.. "The NASA/NSO spectromagnetograph”. Solar Phys., 139: 211-232, 1992.

Judge, P. G., M. Carlsson and K. Wilhelm. "SUMER observations of the quiet solar atmosphere: The network chromosphere and lower transition region". Astrophys. J., 490: L195-L198, 1997.

Kahler, S. W., J. M. Davis and J. W. Harvey. "Comparison of coronal holes observed in soft X-ray and He I 10830 Å spectroheliograms”. Solar Phys., 87: 47-56, 1983.

Kahler, S. W. and H. S. Hudson. "Boundary structures and changes in long-lived coronal holes”. Astrophys. J., 574: 467-476, 2002.

Kaufman, V. and B. Edlén. J. Phys. Chem. Ref. Data, 3: 825, 1974.

Kaufman, V. and W. C. Martin. J. Opt. Soc. Am., 6: 1769, 1989. 
Kaufman, V. and W. C. Martin. J. Phys. Chem. Ref. Data, 20: 83, 1991.

Kaufman, V. and W. C. Martin. J. Phys. Chem. Ref. Data, 22: 279, 1993.

Kelly, R. L. Atomic and ionic spectrum lines below 2000 Angstroms: Hydrogen through Argon. American Institute of Physics (AIP), American Chemical Society and the National Bureau of Standards, New York, 1987.

Kneer, F. "Small-scale waves and motions in photosphere and chromosphere of the Sun". Ann. Geophysicae, 10: 47-53, 1992.

Ko, Y.-K., L. A. Fisk, J. Geiss, G. Gloeckler and M. Guhathakurta. "An Empirical Study of the Electron Temperature and Heavy Ion Velocities in the South Polar Coronal Hole". Solar Phys., 171: 345-361, 1997.

Kohl, J. L., R. Esser, S. R. Cranmer, S. Fineschi, L.D. Gardner et al.. "EUV spectral line profiles in polar coronal holes from 1.3 to $3.0 R_{\odot}$ ". Astrophys. J., 510: L59-L62, 1999.

Kohl, J. L., G. Noci, E. Antonucci and et al. "UVCS/SOHO Empirical Determinations of Anisotropic Velocity Distributions in the Solar Corona”. Astrophys. J., 501: L127L131, 1998.

Kohl, J. L., G. Noci, E. Antonucci, G. Tondello, M. C. E. Huber et al.. "First results from the SOHO ultraviolet coronagraph spectrometer". Solar Phys., 175: 613-644, 1997.

Koutchmy, S. "Study of the June 30, 1973 trans-polar coronal hole". Solar Phys., 51: 399-407, 1977.

Krieger, A. S., A. F. Timothy and E. C. Roelof. "A coronal hole and its identification as the source of a high velocity solar wind stream". Solar Phys., 29: 505-525, 1973.

Laming, J. M., U. Feldman, U. Schüchle, P. Lemaire, W. Curdt et al.. "Electron density diagnostics for the solar upper atmosphere from spectra obtained by SUMER/SOHO”. Astrophys. J., 485: 911-919, 1997.

Landi, E. and M. landini. "The Arcetri Spectral Code for optically thin plasma". Astron. \& Astrophys., 384: 1124-1132, 2002.

Landi, E., H. E. Mason, P. Lemaire and M. landini. "SUMER observations of transition region fine structure". Astron. \& Astrophys., 357: 743-756, 2000.

Landini, M. and B. C. Monsignori Fossi. "The X-UV spectrum of thin plasma". Astron. \& Astrophys. Suppl. Ser., 82: 229-260, 1990.

Lemaire, P., K. Bocchialini and V. Aletti. "Search for signatures of a coronal hole in transition region lines near disk center". Space Sci. Rev., 87: 249-252, 1999. 
Lepping, R. P., M. H. Acuna, L. F. Burlaga, W. M. Farrell, J. A. Slavin et al. . "The WIND Magnetic Field Investigation”. Space Sci. Rev., 71: 207-229, 1995.

Levine, R. H. "Evolution of open magnetic structures on the Sun: the Skylab period". Astrophys. J., 218: 291-305, 1977.

Li, X. "Heating in coronal funnels by ion cyclotron waves". Astrophys. J., 571: L67-L70, 2002.

Li, X., S. R. Habbal, J. L. Kohl and G. Noci. "The effect of temperature anisotropy on observations of Doppler dimming and pumping in the inner corona". Astrophys. J., 501: L133-L137, 1998.

Lie-Svendsen, Ø., V. H. Hansteen, E. Leer and T. E. Holzer. "The effect of transition region heating on the solar wind from coronal holes". Astrophys. J., 566: 562-576, 2002.

Mariska, J. T. "Observational signatures of loop flows driven by asymmetric heating". Astrophys. J., 334: 489-493, 1988.

Mariska, J. T. The Solar Transition Region, volume 23 of Combridge Astrophysics Series. Cambridge University Press, 1992.

Mariska, J. T. and J. P. Boris. "Dynamics and spectroscopy of asymmetrically heated coronal loops". Astrophys. J., 267: 409-420, 1983.

Mariska, J. T., U. Feldman and G. A. Doschek. "Measurements of extreme-ultraviolet emission-line profiles near the solar limb”. Astrophys. J., 226: 698-705, 1978.

Marsch, E. "Kinetic physics of the solar wind, II". In: R. Schwenn and E. Marsch, eds., "Physics of the Inner Heliosphere", pages 45-133. Springer-Verlag, Heidelberg, Germany, 1991.

Marsch, E. "On the possible role of plasma waves in the heating of the chromosphere and corona". In: E. Marsch and R. Schwenn, eds., "Solar Wind Seven", pages 65-68. Pergamon Press, Oxford, England, 1992.

Marsch, E. "Solar wind models from the Sun to $1 \mathrm{AU}$ : constraints by in situ and remote sensing measurements". Space Sci. Rev., 87: 1-24, 1999.

Marsch, E., W. I. Axford and J. F. McKenzie. "Solar wind". In: B. Dwivedi, ed., "The Dynamic Sun", page (in press). Combridge University Press, 2003.

Marsch, E., C. K. Goertz and A. K. Richter. "Wave heating and acceleration of solar wind ions by cyclotron resonance”. J. Geophys. Res., 87: 5030-5044, 1982.

Marsch, E. and A. K. Richter. "HELIOS observational constraints on solar wind expansion". J. Geophys. Res., 89: 6599-6612, 1984. 
Marsch, E. and C.-Y. Tu. "Solar wind and chromospheric network". Solar Phys., 176: 87-106, 1997.

Marsch, E., C.-Y. Tu, K. Wilhelm, W. Curdt, U. Schühle et al.. "Bright plumes and dark lanes as observed in Mg X $625 \AA$ and N V $1239 \AA \AA$ in the solar polar corona". Proc. 5th SOHO Workshop, ESA SP-404: 555-560, 1997.

Mason, H. E. and B. C. Monsignori Fossi. "Spectroscopic diagnostics in the VUV for solar and stellar plasmas". Astron. \& Astrophys. Rev., 6: 123-179, 1994.

McClymont, A. N. “Can cool loop flows explain 'transition region' redshifts?” Astrophys. J., 347: L47-L50, 1989.

McClymont, A. N. and I. J. D. Craig. "Fast downflows in the solar transition region explained". Astrophys. J., 312: 402-411, 1987.

McComas, D. J., B. L. Barraclough, H. O. Funsten, J. T. Gosling, E. Santiago-Munoz et al.. "Solar wind observations over Ulysses' first full polar orbit". J. Geophys. Res., 105: 10419-10433, 2000.

McIntosh, S. W., D. A. Diver, P. G. Judge, P. Charbonneau, J. Ireland et al.. "Spectral decomposition by genetic forward modelling”. Astron. \& Astrophys. Suppl. Ser., 132: 145-153, 1998.

Miralles, M. P., S. R. Cranmer and J. L. Kohl. "Ultraviolet coronagraph spectrometer observations of a high-latitude coronal hole with high oxygen temperatures and the next solar cycle polarity". Astrophys. J., 560: L193-L196, 2001a.

Miralles, M. P., S. R. Cranmer, A. V. Panasyuk, M. Romoli and J. L. Kohl. "Comparison of empirical models from polar and equatorial coronal holes". Astrophys. J., 549: L257L260, 2001b.

Mullan, D. J. and I. A. Ahmad. "Coronal holes: mass loss driven by magnetic reconnection”. Solar Phys., 75: 347-350, 1982.

Mullan, D. J. and W. L. Waldron. "Velocity fields in a low-latitude coronal hole: results from the SOLAR MAXIMAM MISSION". Astrophys. J., 317: 487-501, 1987.

Munro, R. H. and G. L. Withbroe. "Properties of coronal hole derived from extremeultraviolet observations". Astrophys. J., 176: 511-520, 1972.

Neugebauer, M., R. J. Forsyth, A. B. Galvin, K. L. Harvey, J. T. Hoeksema et al.. "Spatial structure of the fast solar wind and comparisons with solar data and models". $J$. Geophys. Res., 103: 14587-14599, 1998.

Neugebauer, M. and C. W. Snyder. "Mariner 2 observations of the solar wind 1. Average properties". J. Geophys. Res., 71: 4469-4484, 1966. 
Neupert, W. M. and V. Pizzo. "Solar coronal holes as sources of recurrent geomagnetic disturbances”. J. Geophys. Res., 79: 3701-3709, 1974.

Newkirk, G. and J. Harvey. "Coronal polar plumes”. Solar Phys., 3: 321-343, 1968.

Noci, G. "Energy budget in coronal holes". Solar Phys., 28: 403-407, 1973.

Nolte, J. T., A. S. Krieger, A. F. Timothy, R. E. Gold, E. C. Goelof et al. . "Coronal holes as sources of solar wind". Solar Phys., 46: 303-322, 1976.

Noyes, R. W. The Sun, Our Star. Harvard University Press, Cambridge, 1982.

Ogilvie, K. W., D. J. Chornay, R. J. Fritzenreiter, F. Hunsaker, J. Keller et al.. "SWE, a comprehensive plasma instrument for the WIND spacecraft". Space Sci. Rev., 71: 55-77, 1995.

Orrall, F. Q., G. J. Rottman and J. A. Klimchuk. "Outflow from the Sun's polar corona". Astrophys. J., 266: L65-L68, 1983.

Papaiannis, M.D. and K.B. Baker. "Determination and analysis of coronal hole radio spectra". Solar Phys., 79: 365-374, 1982.

Parker, E. N. "Dynamics of the interplanetary gas and magnetic fields". Astrophys. J., 128: 664-676, 1958.

Parker, E. N. "Heating solar coronal holes”. Astrophys. J., 372: 719-727, 1991.

Patsourakos, S., J. C. Vial, A. H. Gabriel and N. Bellamine. "Transition-region network boundaries in the quiet Sun: width variation with temperature as observed with CDS on SOHO”. Astrophys. J., 522: 540-546, 1999.

Peter, H. "Analysis of transition-region emission-line profiles from full-disk scans of the Sun using the SUMER instrument on SOHO”. Astrophys. J., 516: 490-504, 1999.

Peter, H. "Multi-component structure of solar and stellar transition regions". Astron. \& Astrophys., 360: 761-776, 2000.

Peter, H. "On the nature of the transition region from the chromosphere to the corona of the Sun”. Astron. \& Astrophys., 374: 1108-1120, 2001.

Peter, H. and P. G. Judge. "On the doppler shifts of solar ultraviolet emission lines". Astrophys. J., 522: 1148-1166, 1999.

Pneuman, G. W. and R. A. Kopp. "Gas-Magnetic Field Interactions in the Solar Corona". Solar Phys., 18: 258-270, 1971.

Pneuman, G. W. and R. A. Kopp. "Downflow in the Supergranulation Network and Its Implications for Transition Region Models”. Solar Phys., 57: 49-64, 1978. 
Reale, F., G. Peres and S. Serio. "Radiatively-driven downdrafts and redshifts in transition region lines. I. Reference model”. Astron. \& Astrophys., 316: 215-228, 1996.

Reale, F., G. Peres and S. Serio. "Radiatively driven downdrafts and redshifts in transition region lines. II. Exploring the parameter space". Astron. \& Astrophys., 318: 506-520, 1997.

Reeves, E. M. "The EUV chromospheric network in the quiet Sun". Solar Phys., 46: 53-72, 1976.

Rottman, G. J., D. M. Hassler, M. D. Jones and F. Orrall. "The systematic radial downflow in the transition region of the quiet sun from limb-to-limb observations of the C IV resonance lines". Astrophys. J., 358: 693-697, 1990.

Rottman, G. J., J. A. Klimchuk and F. Q. Orrall. "Measurement of systematic outflow from the solar transition region underlying a coronal hole". Astrophys. J., 247: L135L138, 1981.

Rottman, G. J., F. Q. Orrall and J. A. Klimchuk. "Measurements of outflow from the base of solar coronal holes". Astrophys. J., 260: 326-337, 1982.

Saito, K. "Polar rays of the solar corona. II". Publ. Astron. Soc. Japan, 17: 1-26, 1965.

Sanchez-Ibarra, A. "Longitudinal and temporal variations of sunspot regions and coronal holes during cycle 21”. Solar Phys., 125: 125-132, 1990.

Sandbæk, Ø., E. Leer and V. H. Hansteen. "On the relation between coronal heating, flux tube divergence, and the solar wind proton flux and flow speed". Astrophys. J., 436: 390-399, 1994.

Scherrer, P. H., R. S. Bogart, R. I. Bush, J. T. Hoeksema, A. G. Kosovichev et al.. "The Solar Oscillations Investigation - Michelson Doppler Imager". Solar Phys., 162: 129_ $188,1995$.

Schmieder, B., P. Heinzel, T. Kucera and J.-C. Vial. "Filament observations with SOHO instruments SUMER/CDS: The behaviour of hydrogen Lyman lines". Solar Phys., 181: 309-326, 1998.

Schühle, U., W. Curdt, K. Wilhelm, S.K. Solanki and K. Stucki. "Signatures of coronal hole spectra between $660 \AA$ and $1460 \AA$ measured with SUMER on SOHO”. Space Sci. Rev., 87: 299-302, 1999.

Schwenn, R. "Large-scale structure of the interplanetary medium". In: R. Schwenn and E. Marsch, eds., "Physics of the Inner Heliosphere, I", pages 99-182. Springer-Verlag, Heidelberg, Germany, 1990. 
Schwenn, R., B. Inhester, S. P. Plunkett, A. Epple, B. Podlipnik et al.. "First view of the extended green-line emission corona at solar activity minimum using the LASCO-C1 coronagraph on SOHO”. Solar Phys., 175: 667-684, 1997.

Sheeley, N. R. Jr. "Polar faculae during the interval 1906-1975". J. Geophys. Res., 81: 3462-3464, 1976.

Sheeley, N. R. Jr., J. W. Harvey and W. C. Feldman. "Coronal holes, solar wind streams, and recurrent geomagnetic disturbances - 1973-1976”. Solar Phys., 49: 271-278, 1976.

Sittler, E. C. and M. Guhathakurta. "Semiempirical two-dimensional magnetohydrodynamic model of the solar corona and interplanetary medium". Astrophys. J., 523: 812826, 1999.

Snyder, C. W. and M. Neugebauer. "The solar wind velocity and its correlation with cosmic ray variations and with solar and geomagnetic activity". J. Geophys. Res., 68: 6361-, 1963.

Spadaro, D., S. K. Antiochos and J. T. Mariska. "Nonequilibrium ionization effects in asymmetrically heated loops". Astrophys. J., 382: 338-343, 1991.

Stenborg, G., R. Schwenn and B. Inhester N. Srivastava. "On the rotation rate of the solar corona". ESA SP Series, SP-448: 1107-1113, 1999.

Sterling, A. C. "Solar spicules: A review of recent models and targets for future observations". Solar Phys., 196: 79-111, 2000.

Stucki, K., S. K. Solanki, C. D. Pike, U. Schühle and I. Rüedi. "Properties of ultraviolet lines observed with the Coronal Diagnostic Spectrometer (CDS/SOHO) in coronal holes and the quiet Sun". Astron. \& Astrophys., 381: 653-667, 2002.

Stucki, K., S. K. Solanki, I. Rüedi, J.O. Stenflo, A. Brkovic et al.. "Coronal holes versus normal quiet Sun observed with SUMER”. Astrophys. Space Sci., 264: 53-61, 1999.

Stucki, K., S. K. Solanki, U. Schühle and I. Rüedi. "On the relationship between shift and intensity of ultraviolet lines in coronal holes and the quiet Sun". Astron. \& Astrophys., 362: L49-L52, 2000a.

Stucki, K., S. K. Solanki, U. Schühle, I. Rüedi and K. Wilhelm. "Comparison of farultraviolet emission lines formed in coronal holes and the quiet Sun". Astron. \& Astrophys., 363: 1145-1154, 2000b.

Svalgaard, L., T. L. Duvall and P. H. Scherrer. "The strength of the sun's polar fields". Solar Phys., 58: 225-239, 1978.

Teriaca, L., D. Banerjee and J. G. Doyle. "SUMER observations of Doppler shift in the quiet Sun and in an active region". Astron. \& Astrophys., 349: 636-648, 1999. 
Timothy, A. F., A. S. Krieger and G. S. Vaiana. "The Structure and Evolution of coronal holes". Solar Phys., 42: 135-156, 1975.

Tousey, R., J.-D. Bartoe, J. D. Bohlin, G. E. Brueckner, J. D. Purcell et al. . "A Preliminary Study of the Extreme Ultraviolet Spectroheliograms from Skylab". Solar Phys., 33: 265-280, 1973.

Tsiropoula, G., C. E. Alissandrakis and B. Schmieder. "The fine structure of a chromospheric rosette". Astron. \& Astrophys., 271: 574-586, 1993.

Tu, C.-Y., E. Marsch and K. Wilhelm. "Ion temperatures as observed in a solar coronal hole". Space Sci. Rev., 87: 331-334, 1999.

Tu, C.-Y., E. Marsch, K. Wilhelm and W. Curdt. "Ion temperatures in a solar polar coronal hole observed by SUMER on SOHO”. Astrophys. J., 503: 475-482, 1998.

Vaiana, G. S., A. S. Krieger, L. P. Van Speybroeck and T. Zehnpfennig. Bull. Am. Phys. Soc., 15: 611, 1970.

Vernazza, J. E., E. H. Avrett and R. Loeser. "Structure of the solar chromosphere. I. Basic computations and summary of the results". Astrophys. J., 184: 605-631, 1973.

Vocks, C. and E. Marsch. "Kinetic results for ions in the solar corona with wave-particle interactions and Coulomb collisions”. Astrophys. J., 568: 1030-1042, 2002.

Wagner, W. J. "Solar rotation as marked by extreme-ultraviolet coronal holes". Astrophys. J., 198: L141-L144, 1975.

Waldmeier, M. Die Sonnenkorona (II). Birkhäuser Verlag Basel und Stuttgart, 1957.

Waldmeier, M. "The coronal hole at the 7 March 1970 solar eclipse". Solar Phys., 40: 351-358, 1975.

Walker, A. B. C. Jr., C. E. Deforest, R. B. Hoover and T. W. Jr. Barbee. "Thermal and density structure of polar plumes". Solar Phys., 148: 239-252, 1993.

Wang, Y. M. "Polar plumes and the solar wind". Astrophys. J., 435: L153-L156, 1994.

Wang, Y. M. "Empirical relationship between the magnetic field and the mass and energy flux in the source regions of the solar wind". Astrophys. J., 449: L157-L160, 1995.

Wang, Y. M. "Network activity and the evaporative formation of polar plumes". Astrophys. J., 501: L145-L150, 1998.

Wang, Y. M., S. H. Hawley and N. R. Jr. Sheeley. "The magnetic nature of coronal holes". Science, 271: 464-469, 1996.

Wang, Y. M. and N. R. Jr. Sheeley. "Coronal plumes and their relationship to network activity”. Astrophys. J., 452: 457-461, 1995. 
Wang, Y. M., N. R. Jr. Sheeley, K. P. Dere, R. T. Duffin, R. A. Howard et al.. "Association of Extreme-ultraviolet Imaging Telescope (EIT) polar plumes with mixed-polarity magnetic network". Astrophys. J., 484: L75-L78, 1997.

Warren, H. P. and D. M. Hassler. "The density structure of a solar polar coronal hole". $J$. Geophys. Res., 104: 9781-9789, 1999.

Warren, H. P., J. T. Mariska and K. Wilhelm. "Observations of Doppler shifts in a solar polar coronal hole”. Astrophys. J., 490: L187-L190, 1997a.

Warren, H. P., J. T. Mariska, K. Wilhelm and P. Lemaire. "Doppler shifts and nonthermal broadening in the quiet solar transition region: O vI". Astrophys. J., 484: L91-L94, $1997 b$.

Warren, H. P. and A. R. Winebarger. "Small scale structure in the solar transition region". Astrophys. J., 535: L63-L66, 2000.

Wikst $\varnothing 1, \varnothing .$, V. H. Hansteen and M. Carlsson. "Chromospheric and transition region internetwork oscillations: A signature of upward- propagating waves". Astrophys. J., 531: 1150-1160, 2000.

Wikst $\varnothing 1, \varnothing$., P. G. Judge and V. H. Hansteen. "Signatures of acoustic and magnetic waves in solar and stellar coronae". Astrophys. J., 483: 972-983, 1997.

Wilhelm, K. "The darkest regions of solar polar coronal holes observed by SUMER on SOHO”. Astrophys. Space Sci., 264: 43-52, 1999.

Wilhelm, K. "Solar spicules and macrospicules observed by SUMER". Astron. \& Astrophys., 360: 351-362, 2000.

Wilhelm, K. "Past and recent observations of the solar upper atmosphere at vacuumultraviolet wavelengths". J. Atmos. Solar-Terr. Phys., 65: 167-189, 2003.

Wilhelm, K., W. Curdt, E. Marsch, U. Schühle, P. Lemaire et al.. "SUMER-Solar ultraviolet measurements of emitted radiation". Solar Phys., 162: 189-231, 1995.

Wilhelm, K., I. E. Dammasch, E. Marsch and D. M. Hassler. "On the source regions of the fast solar wind in polar coronal holes”. Astron. \& Astrophys., 353: 749-756, 2000.

Wilhelm, K., I. E. Dammasch and L. D. Xia. "Observations of ultraviolet emission lines in solar coronal holes on the disk with SUMER on SOHO”. Adv. Space Res., 30: 517-522, 2002.

Wilhelm, K., P. Lemaire, W. Curdt, U. Schühle, E. Marsch et al.. "First results of the SUMER telescope and spectrometer on SOHO I: Spectra and spectroradiometry". Solar Phys., 170: 75-104, 1997. 
Wilhelm, K., P. Lemaire, I. E. Dammasch, J. Hollandt, U. Schühle et al.. "Solar irradiances and radiances of UV and EUV lines during the minimum of sunspot activity in 1996". Astron. \& Astrophys., 334: 685-702, 1998a.

Wilhelm, K., E. Marsch, B. N. Dwivedi, D. M. Hassler, P. Lemaire et al.. "The solar corona above polar coronal holes as seen by SUMER on SOHO”. Astrophys. J., 500: 1023-1038, 1998b.

Winebarger, A. R., A. Gordon Emslie, J. T. Mariska and H. P. Warren. "Energetics of explosive events observed with SUMER”. Astrophys. J., 565: 1298-1311, 2002.

Withbroe, G. L. "The chromospheric and transition layers in coronal holes". In: J.B. Zirker, ed., "Coronal holes and high speed wind streams", pages 145-177. Colorado Associated University Press, Boulder, 1977.

Withbroe, G. L. "The role of spicules in heating the solar atmosphere: Implications of EUV observations". Astrophys. J., 267: 825-836, 1983.

Withbroe, G. L. "The temperature structure, mass, and energy flow in the corona and inner solar wind". Astrophys. J., 325: 442-467, 1988.

Withbroe, G. L. and R. W. Noyes. "Mass and energy flow in the solar chromosphere and corona". Ann. Rev. Astron. Astrophys., 15: 363-387, 1977.

Woch, J., W. I. Axford, U. Mall, B. Wilken, S. Livi et al.. "SWICS/Ulysses observations: The three-dimensional structure of the heliosphere in the declining/minimum phase of the solar cycle". Geophys. Res. Lett., 24: 2885-2888, 1997.

Young, P. R. and R. Esser. "Temperature and density in coronal holes - results from CDS/SOHO". In: S. R. Habbal and et al., eds., "Solar Wind Nine", pages 273-276. The American Institute of Physics, USA, 1999.

Young, P. R., J. A. Klimchuk and H. E. Mason. "Temperature and density in a polar plume - measurements from CDS/SOHO”. Astron. \& Astrophys., 350: 286-301, 1999.

Zirker, J. B. Coronal holes and high speed wind streams. Colorado Associated University Press, Boulder, 1977.

Zwaan, C. "Elements and patterns in the solar magnetic field". Ann. Rev. Astron. Astrophys., 25: 83-111, 1987. 


\section{Danksagung}

Die vorliegende Arbeit wurde am Max-Planck-Institut für Aeronomie (MPAe) in Katlenburg-Lindau angefertigt. Danken möchte ich den Direktoren des Instituts für die Bereitstellung des Arbeitsplatzes und für die Unterstützung bei der Teilnahme an internationalen Konferenzen.

Danken möchte ich ebenso dem Deutschen Akademischen Austauschdienst (DAAD) für die Gewährung des Stipendiums und für die Hilfe bei meinem Aufenthalt in Deutschland.

Insbesondere danken möchte ich Herrn Prof. Dr. E. Marsch für die Betreuung dieser Dissertation, für die zahlreichen Diskussionen, und für das Korrekturlesen des Manuskripts, für die er sich stets viel Zeit genommen hat.

Ganz herzlich danken möchte ich ebenso Herrn Prof. Dr. F. Kneer für die Bemühung um die Zulassung zur Promotion, für die Hilfe bei dem Antrag des DAAD-stipendiums, und für die Betreuung der Arbeit seitens der Universität Göttingen.

Herzlicher Dank gilt den gesamten SUMER-Gruppe des Max-Planck-Institutes für Aeronomie für die Bereitstellung der Daten und Auswertungssoftware. Insbesondere danken möchte ich ehemaligen PI Herrn Dr. K. Wilhelm und gegenwärtigen PI Herrn Dr. W. Curdt für die hilfreichen Diskussionen und die gute Zusammenarbeit.

Mein Dank gilt auch den zahlreichen Menschen von IMPRS, insbesondere Herrn Dr. D. Schmitt, für die vielfältige Hilfen sowie für die freundliche Arbeitsatmosphäre.

Für die vielfältige Unterstützung danke ich auch Herrn Dr. J.-S. Wang sowie seiner Familie, Herrn Prof. C.-Y. Tu, Herrn Dr. Q.-G. Zong, Frau Dr. S.-Y. Fu, und allen anderen Freunden.

Liebevoller Dank gilt nicht zuletzt natürlich meiner Frau Minlan und unserer Tochter Yiling für ihre Begleitung mit mir in Deutschland, und auch für die vielfach geartete Unterstützung.

Data use acknowledgments: The SUMER project is financially supported by DLR, CNES, NASA and the ESA PRODEX program (Swiss contribution). I'd like to thank the MDI, EIT, SWE and MFI teams for use of their data. SUMER, EIT and MDI are part of SOHO, the Solar and Heliospheric Observatory of ESA and NASA. SWE and MFI are part of WIND mission. NSO/Kitt Peak data used in this work are produced cooperatively by NSF/NOAO, NASA/GSFC, and NOAA/SEL. 



\section{Lebenslauf}

\section{Persönliche Daten}

$\begin{array}{ll}\text { Name: } & \text { Xia } \\ \text { Vorname: } & \text { Lidong } \\ \text { Geburtsdatum: } & \text { 10. November 1967 } \\ \text { Geburtsort: } & \text { Kaihua, V. R. China } \\ \text { Staatsangehörigkeit: } & \text { chinesisch } \\ \text { Familienstand: } & \text { verheiratet, 1 Kind }\end{array}$

\section{Schulbildung}

Sept. 1974 - Juli 1979 Grundschule in Kaihua, Provinz Zhejiang

Sept. 1979 - Juli 1985 Mittelschule in Kaihua, Provinz Zhejiang

\section{Studium}

Sept. 1985 - Juli 1990 Studium in Physik, Chinesische Universität der Technik und Wissenschaften (USTC), Hefei

Schwerpunkt: Weltraumphysik

Abschluß: "Bachelor of Science"

Sept. 1990 - Juni 1993 Diplomarbeit, Chinesische Universität der Technik und Wissenschaften, Hefei

Schwerpunkt: Weltraumphysik

Abschluß: "Master of Science"

Seit Oktober 1999

DAAD-Stipendiat, Max-Planck-Institut für Aeronomie, Katlenburg-Lindau

\section{Berufstätigkeit}

Juli 1993 - Juni 1995

Juli 1995 - Mai 1999
Assistent, Chinesische Universität der Technik und Wissenschaften, Hefei

Dozent, Chinesische Universität der Technik und Wissenschaften, Hefei 